\title{
Role of pharmacokinetic modeling and simulation in precision dosing of anticancer drugs
}

DOI:

10.21037/tcr.2017.09.14

\section{Document Version}

Accepted author manuscript

Link to publication record in Manchester Research Explorer

\section{Citation for published version (APA):}

Darwich, A., Ogungbenro, K., Hatley, O. J. D., \& Rostami-Hochaghan, A. (2017). Role of pharmacokinetic modeling and simulation in precision dosing of anticancer drugs. Translational Cancer Research. https://doi.org/10.21037/tcr.2017.09.14

\section{Published in:}

Translational Cancer Research

\section{Citing this paper}

Please note that where the full-text provided on Manchester Research Explorer is the Author Accepted Manuscript or Proof version this may differ from the final Published version. If citing, it is advised that you check and use the publisher's definitive version.

\section{General rights}

Copyright and moral rights for the publications made accessible in the Research Explorer are retained by the authors and/or other copyright owners and it is a condition of accessing publications that users recognise and abide by the legal requirements associated with these rights.

\section{Takedown policy}

If you believe that this document breaches copyright please refer to the University of Manchester's Takedown Procedures [http://man.ac.uk/04Y6Bo] or contact uml.scholarlycommunications@manchester.ac.uk providing relevant details, so we can investigate your claim.

\section{OPEN ACCESS}




\section{Translational Cancer Research}

--Manuscript Summary--

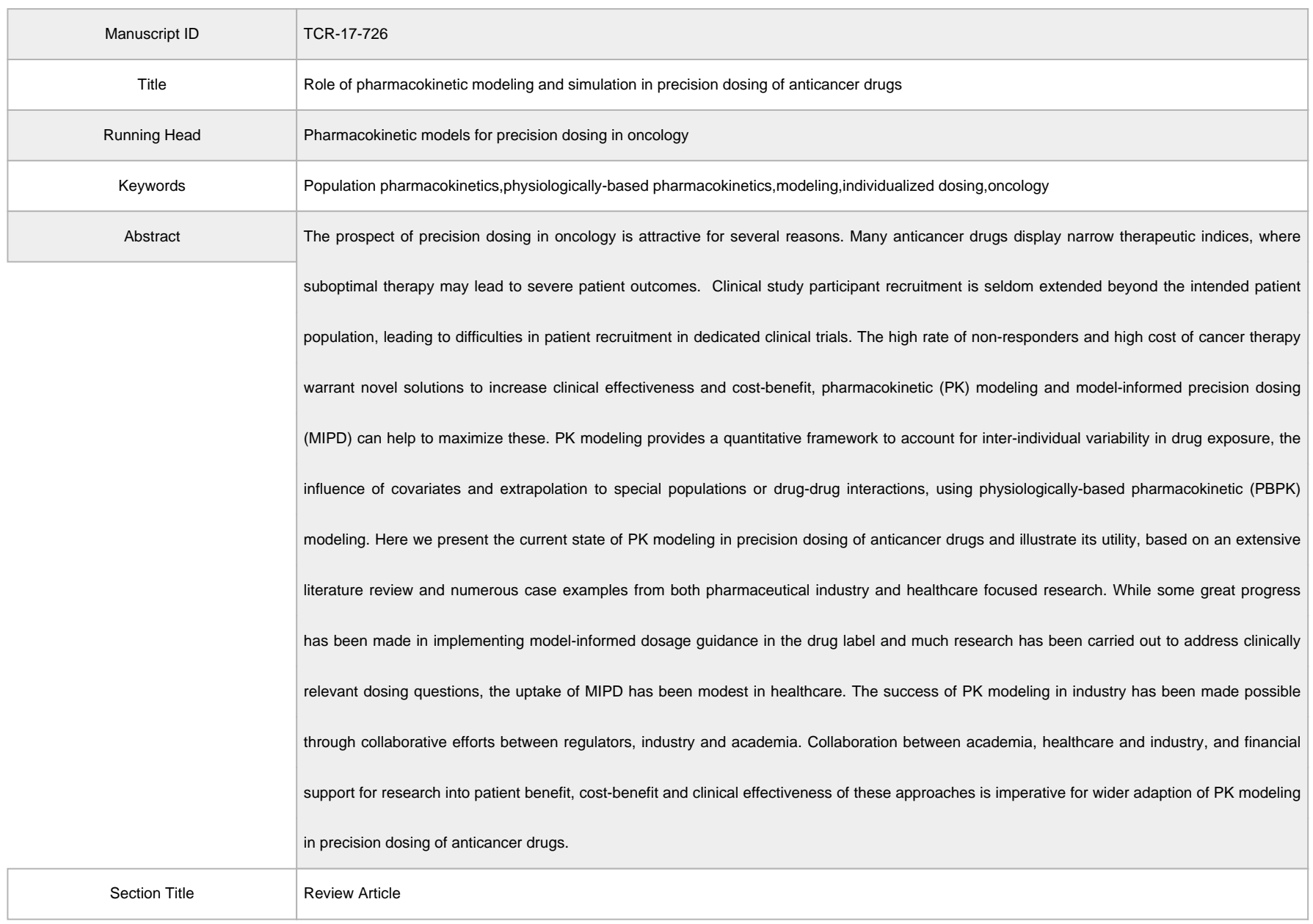




\section{Role of pharmacokinetic modeling and simulation in precision dosing of anticancer drugs}

Adam S. Darwich ${ }^{1}$, Kayode Ogungbenro ${ }^{1}$, Oliver J.D. Hatley ${ }^{2}$, Amin Rostami-

Hodjegan $^{1,2}$

${ }^{I}$ Centre for Applied Pharmacokinetic Research, Division of Pharmacy \& Optometry,

School of Health Sciences, Faculty of Biology, Medicine \& Health,

The University of Manchester, Manchester, UK.

${ }^{2}$ Certara, Blades Enterprise Building, Sheffield, UK.

Corresponding author:

Adam S. Darwich,

The University of Manchester,

Room 3.123, Stopford Building,

Oxford Road,

Manchester M13 9PT,

United Kingdom

Tel: +44(0)161-2752399

Fax: +44(0)161-2758349

Email: adam.darwich@manchester.ac.uk 
Pharmacokinetic models for precision dosing in oncology

\section{Author contribution:}

(1) Conception and design: $A S D, K O, O J D H, A R H$.

(2) Administrative support: $A S D, K O, O J D H, A R H$.

(3) Provision of study materials or patients: ASD, KO, OJDH, ARH.

(4) Collection and assembly of data: ASD, KO, OJDH.

(5) Data analysis and interpretation: $A S D, K O, O J D H, A R H$.

(6) Manuscript writing: ASD, KO,OJDH, ARH.

(7) Final approval of manuscript: ASD, KO, OJDH, ARH.

Running title: Pharmacokinetic models for precision dosing in oncology

Keywords: Population pharmacokinetics, physiologically-based pharmacokinetics, modeling, individualized dosing, oncology.

Word count: 5418 


\section{Abstract}

The prospect of precision dosing in oncology is attractive for several reasons. Many anticancer drugs display narrow therapeutic indices, where suboptimal therapy may lead to severe patient outcomes. Clinical study participant recruitment is seldom extended beyond the intended patient population, leading to difficulties in patient recruitment in dedicated clinical trials. The high rate of non-responders and high cost of cancer therapy warrant novel solutions to increase clinical effectiveness and cost-benefit, pharmacokinetic (PK) modeling and model-informed precision dosing (MIPD) can help to maximize these. PK modeling provides a quantitative framework to account for interindividual variability in drug exposure, the influence of covariates and extrapolation to special populations or drug-drug interactions, using physiologically-based pharmacokinetic (PBPK) modeling. Here we present the current state of PK modeling in precision dosing of anticancer drugs and illustrate its utility, based on an extensive literature review and numerous case examples from both pharmaceutical industry and healthcare focused research. While some great progress has been made in implementing model-informed dosage guidance in the drug label and much research has been carried out to address clinically relevant dosing questions, the uptake of MIPD has been modest in healthcare. The success of PK modeling in industry has been made possible through collaborative efforts between regulators, industry and academia. Collaboration between academia, healthcare and industry, and financial support for research into patient benefit, cost-benefit and clinical effectiveness of these approaches is imperative for wider adaption of PK modeling in precision dosing of anticancer drugs. 


\section{Background}

In drug development, late phase clinical trials often aim to establish uniform dosing, balancing efficacy and toxicity, across the patient population from a limited set of proposed dosage schemes (1). Dosing of anticancer drugs has traditionally been based on body surface area (BSA) under the assumption that there is a relationship between BSA and clearance $(C L)$ or volume of distribution $\left(V_{d}\right)$. However, this relationship is in many instances poor and may therefore not accurately reflect the change in drug exposure seen across the population (2-5), meaning variability in drug exposure may remain high at the established dosage regimen (5). This is particularly true when the drug is dosed in a more diverse patient population in clinical practice, such as: complex drug-drug interactions (DDIs), pediatric patients, renally/hepatically impaired or other special populations (6). Explicit dosage recommendations are often absent from the drug label for most special populations at the time of approval (7). These factors contribute to variable clinical practices, where clinicians are challenged to make decisions based on experience and the many times limited literature. Patients with multiple comorbidities/co-medication are therefore at risk of suboptimal pharmacotherapy that may lead to unacceptable levels of toxicity or reduced efficacy (6, 8, 9). Model-informed precision dosing (MIPD) provides a quantitative framework for achieving the accurate dose for the individual patient through statistical and/or mathematical modeling, such as pharmacokinetic (PK) modeling, by accounting for inter-individual variability (IIV), and other factors that lead to variable drug exposure and/or pharmacodynamic (PD) response (10). 
Here we examine the current state of PK modeling in dose individualization of anticancer drugs. The comparative analysis presented here was based on a sample of 393 peer-reviewed publications on pharmacokinetic modeling in oncology (see Table A1, in Supplementary Appendix available online). The dataset should not be considered an exhaustive list of the abundant literature on PK modeling in oncology. Many arguments on precision dosing presented here are part of a much broader discussion on MIPD across therapeutic areas (7, 10-12).

\section{The case for model-informed precision dosing in oncology}

The traditional resolution to dose optimization in special populations/DDIs is to carry out dedicated clinical studies. This is however not always feasible in oncology due to patient recruitment issues around dosing drugs in vulnerable populations or patients outside the indicated treatment group (13). Statistical nonlinear-mixed effects (NLME) modeling (population-pharmacokinetic/pharmacodynamic modeling, or pop-PK/PD) aims to describe the IIV in PK parameters using compartmental and increasingly mechanistic models. Physiologically-based pharmacokinetic (PBPK) modeling and simulation (M\&S), attribute physiological meaning to PK models by mimicking physiology (inter-compartmental clearances informed by blood flows, volumes based on organ/tissue volumes etc.) in an attempt to better understand the processes that determine drug ADME (absorption, distribution, metabolism and excretion). The combined approach ('middle-out') accommodates physiological models where model parameters may account for observed IIV in the population sample (See Figure 1) (14). Pop-PK/PD and PBPK M\&S have gained increasing acceptance in pharmaceutical 
research and development $(\mathrm{R} \& \mathrm{D})$ and by regulatory agencies over the last couple of decades to a point where dedicated clinical trials may be substituted/supplemented by modeling, foremost interpolating the effect of metabolic DDIs (15). It is anticipated that M\&S will gain further utility over the coming years as confidence is built in other areas of application, both in pharmaceutical $\mathrm{R} \& \mathrm{D}$, regulatory submission and clinical practice for dose individualization $(7,10,12)$.

Dose individualization, personalized dosing or precision dosing, may be considered part of the well-recognized paradigm of precision medicine. Precision medicine pursues to personalize prevention, diagnostics and optimal treatment of disease based on individual patient characteristics e.g., genotyping, renal function and other biomarkers (16). Similarly, precision dosing strives to account for between-patient variability in drug exposure and response to optimize dosing for the individual. This is not a novel idea, carboplatin is the perhaps most famous example in oncology, seeing early adoption of renal function guided dosing (Calvert et al. formula) to reduce the risk of hematological toxicity (17). Similarly, PK-based dose adjustment, using therapeutic drug monitoring (TDM), of 5-fluorouracil was shown to produce superior treatment response and reduced toxicity as compared to BSA guided dosing alone in metastatic colorectal cancer (18).

Precision dosing in cancer therapy is attractive for several reasons. Many anticancer drugs display narrow therapeutic indices, where suboptimal therapy may lead to severe patient outcomes. Clinical study participant recruitment issues accentuates the 
difficulty of patient recruitment in dedicated clinical trials for special populations and is perhaps part of the reason why there has been an above average adoption of PBPK M\&S for new drug applications (NDAs) in oncology to the US Food and Drug Administration (FDA) (19). The relatively high rate of non-responders in cancer treatment together with high cost of cancer therapies warrants alternative approaches to increasing patient benefit and cost-benefit; this may include more effective use of approaches that maximize treatment outcome, such as PK modeling and MIPD (20, 21).

\section{Application of pharmacokinetic modeling in oncology}

Model-informed drug discovery and development has become established practice in the pharmaceutical industry over the past decades, where today it is employed across drug development to inform internal and regulatory decisions $(15,22)$. In early discovery and pre-clinical development, modeling is used to inform candidate selection, ADME characterization, translation of exposure and effect and more, this includes popPK/PD, PBPK and more mechanistic systems pharmacology/biology approaches. Pop$\mathrm{PK} / \mathrm{PD}$ is widely used in clinical development to investigate efficacy, dose selection and dose bridging. PBPK M\&S is used clinically for predicting metabolic DDIs, impact of genetic polymorphism, biopharmaceutics effects and extrapolating to special populations (22).

Analysis of peer-reviewed publications using PK modeling in oncology, based on the modeling approach (see Figure 2), showed that a majority of studies employed 
population-based approaches in their data analysis (75\%), a subset of these include traditional pop-PK (45\%), pop-PK/PD (14\%), Bayesian pop-PK (10\%) and semimechanistic pop-PK/PD (6\%). PBPK M\&S accounted for $8 \%$ of identified studies. In terms of areas of application of PK modeling (see Figure 3), the most prominent area of application was investigation of covariates (49\%) to account for IIV in PK. This was followed by studies investigating dosing issues (22\%), including dose finding and practice based dosing issues. The most studied special populations included pediatric patients (13\%), hepatic (3\%) and renal impairment (2\%). Other investigated special populations included: pregnancy, elderly, and more. Other areas of investigation included: toxicity (18\%), dose/PK-efficacy studies (response: $8 \%$ ), metabolite kinetics (8\%), metabolic/transporter genotype/phenotype (6\%), DDIs (5\%), limited sampling strategies (5\%) and more.

In PBPK M\&S, predictions of metabolic DDIs and extrapolation to special populations were the perhaps most prominent area of research. This was consistent with common areas of application seen in regulatory submissions where predictions of DDIs tend to dominate due to more well-established body of evidence to support PBPK, guidelines and regulatory acceptance $(15,19)$. There is in other words wide application of PK modeling to address critical questions in dose individualization of oncology drugs.

\section{Population pharmacokinetics and covariate analysis to aid precision}

\section{dosing in oncology}


Pop-PK/PD aims to describe the observed IIV in drug exposure and response for a given population sample. The method allows estimation of the population mean $(\theta)$ and IIV $(\eta)$ of PK/PD parameters and the remaining residual, or unexplained, variability $(\varepsilon)$. The approach allows interpolation of drug exposure and response over the observed parameter space through identification of covariates (demographics, genetic polymorphism and other pathophysiological variables). For example, covariates can be included as either dichotomous (Equation 1) or continuous effects (Equation 2) on PK parameters, e.g., (23):

$$
\theta_{j, i}=\theta_{j} \cdot \theta_{C o v, n}{ }^{C o v, n}+\eta_{j, i}
$$

\section{Equation 1}

$$
\theta_{j, i}=\theta_{j} \cdot\left(\frac{\operatorname{Cov}_{i}}{\operatorname{Cov}}\right)^{\theta_{c o v, n}, n}+\eta_{j, i}
$$

\section{Equation 2}

Where mean effect of the $n^{\text {th }}$ covariate $\left(\theta_{\text {Cov,n }}\right)$ and IIV of the $j^{\text {th }}$ parameter of the $i^{\text {th }}$ individual $\left(\eta_{\mathrm{j}, \mathrm{i}}\right)$ on population mean $\left(\theta_{\mathrm{j}}\right)$ determines the individual parameter estimate for the $\mathrm{j}^{\text {th }}$ parameter $\left(\theta_{\mathrm{j}, \mathrm{i}}\right), \operatorname{Cov}_{\mathrm{i}}$ is the individual observed covariate and $C^{\prime}{ }^{\prime} v$ the central tendency of the sample population. Significant covariates account for IIV in exposure, 
meaning that a combined PK-covariate model can forecast individual exposure based on individual biomarker data prior to dosing and refine predictions following sparse PK sampling or TDM. This makes for a powerful tool for precision dosing. Covariate significance can be determined using e.g., step-wise inclusion based on a predefined statistical criteria (post-hoc $\rho$-value testing for difference in objective function), or alternative approaches. Pop-PK-covariate modeling has been successfully employed for oncology drugs to individualize dosing, as included in drug labelling $(24,25)$. Further there are a number of examples of pop-PK-covariate models that have been used to address dosing issues in clinical practice with some success (See section:

'Pharmacokinetic modeling of anticancer drugs in healthcare').

Figure 4 shows identified PK covariates for anticancer drugs based on the peerreviewed literature. The most commonly included covariates were bodyweight $(50 \%$ of drugs in sample set) and other demographic data (sex [28\%], BSA [26\%] and age [21\%]). Other common covariates included biomarkers related renal function (creatinine clearance $[19 \%]$, serum creatinine $[7 \%]$ and estimated glomerular filtration rate $[5 \%])$, drug-binding plasma proteins (albumin [17\%] and $\alpha_{1}$-acid glycoprotein [AAG; $\left.2 \%\right]$ ), cancer type (14\%) and concomitant treatment (11\%). Other biomarkers of liver function were also reasonably prominent as model covariates, e.g., alanine amino transferase (ALT; 9\%), aspartate amino transferase (AST; 6\%) and alkaline phosphatase (ALK; 5\%) including more. Metabolic genotyping was included for $7 \%$ of drugs. 
It has been recognized that there is a disparity between the wealth of covariates identified in the literature and the limited number pertaining to the dosage recommendation in the drug label of oncology drugs (25). Figure 5 shows factors affecting explicit dose recommendations in the FDA label for selected drugs compared to additional identified PK covariates in the literature. There are many potential explanations for this: covariate selection can be biased (insufficient power, collinearity etc), not all statistically significant covariates are clinically relevant, covariates may for example be of obscure meaning and have little physiological/pharmacological relevance $(25,26)$. Further, the identification of significant but low-effect covariates may have little clinical implication. Here, cut-off points have been proposed where covariates may be considered clinically relevant if they explain at least 20 to $30 \%$ of $\operatorname{IIV}(25,27)$. A lack of communication of research between academia and industry may also affect the difference in the adoption of covariates, suggesting some scope for further individualization of dosing of oncology drugs based on disseminated research (25).

\section{Physiologically-based pharmacokinetic (PBPK) modeling to inform}

\section{individualized dosing of anticancer drugs}

By assigning physiological meaning to model parameters, PBPK M\&S offers a method for quantitative extrapolation of drug exposure from in vitro to in vivo (IVIVE), between species, across populations and for metabolic/transporter DDIs. In oncology, PBPK M\&S has been used extensively for the prediction of DDIs, special populations (renal/hepatic impairment and pediatrics) and biopharmaceutics effects (absorption, 
formulation, food effects). In fact, some of the earliest examples of PBPK M\&S in oncology can be traced back to modeling of chemotherapy agents in the 1970s (34).

There are several reasons why for the wide usage of PBPK in oncology: ethical/safety or recruitment issues, many oncology drugs exhibit narrow therapeutic indices and/or pose risks of severe toxicity and may therefore require more consideration for precision dosing, many anticancer drugs are carried forward through accelerated regulatory approval meaning that studies that have not been carried out in timely fashion may be substituted by PBPK M\&S (19). Numerous examples of PBPK M\&S of oncology drugs exist in the literature, including for: pediatrics (35-37), biopharmaceutics effects (38), renal impairment (39-41), hepatic impairment (42), metabolic phenotypes/genotypes $(43,44)$ and adherence (44) metabolic/transporter DDIs (45-49), with more examples available in FDA drug labels (50). The current view of FDA regarding PBPK-informed dosing, is that sufficient evidence exists to employ verified models for the prediction of metabolic DDIs where the drug is the victim substrate (50). For special populations and biopharmaceutics effects the jury is still out in the absence of more evidence to support the ability to prospectively and quantitatively predict these effects (50). Here we present selected case examples to illustrate the usefulness of PBPK M\&S for dose individualization of anticancer drugs.

\section{Case examples}

Ibrutinib - metabolic drug-drug interactions 
Ibrutinib (Imbruvica ${ }^{\circledR}$; Bruton’s tyrosine kinase inhibitor) was granted accelerated approval by the FDA in 2013 (51). The drug is given orally and undergoes extensive first-pass metabolism, mainly via CYP3A4 and to a lesser extent by CYP2D6, whilst undergoing minimal renal clearance. Ketoconazole inhibited $96 \%$ of ibrutinib's metabolism in human liver microsomes. A PBPK model was developed to evaluate CYP3A4 DDIs in healthy volunteers with ibrutinib as the victim. The model was validated against clinical DDI studies of ibrutinib in the presence of ketoconazole (strong inhibitor) and rifampin (strong inducer). The PBPK model was then used to interpolate DDI effects of mild, moderate and strong CYP3A4 inducers and inhibitors and used to inform dose guidance in the drug label $(52,53)$.

\section{Sonidegib - bridging drug-drug interactions to cancer patients}

Sonidegib $\left(\mathrm{Odomzo}^{\circledR}\right)$ is an oral anticancer agent for the treatment of locally advanced basal cell carcinoma. The drug displays low oral bioavailability. In vitro drug metabolism studies and clinical DDI trials in the presence of ketoconazole and rifampin were carried to elucidate the metabolic contribution of sonidegib elimination. Coadministration of ketoconazole led to a 2.25-fold increase in AUC, whereas rifampin produced a $72 \%$ reduction in exposure. A PBPK model was developed to bridge the DDI data from healthy volunteers to cancer patients at the ratified dose, to extrapolate to steady state effects and interpolate the effect of moderate/weak CYP3A inhibition/induction. The simulation study showed the effect of the DDIs to be slightly reduced in cancer patients compared to healthy and the interaction magnitude to increase at steady state dosing of sonedegib. The simulated DDI magnitudes and 
alternative dosing schedules informed dosing recommendations provided in the FDA drug label (54). The study demonstrates the utility of using PBPK M\&S to bridge the effect of metabolic DDIs from healthy volunteers to cancer patients.

\section{Alectinib - biopharmaceutics effects}

Alectinib (Alecenza ${ }^{\circledR}$; selective anaplastic lymphoma kinase $[$ ALK] inhibitor) underwent accelerated regulatory approval in 2015 because of likely clinical benefit in treating ALK-positive non-small cell lung cancer (55). The drug is a lipophilic basic ( $\mathrm{pKa} \sim 6-7$ ) displaying poor solubility and moderate oral bioavailability. As alectinib is given orally the $\mathrm{pH}$-dependent solubility may be indicative of potential impact of biopharmaceutics effects. Parrott, and co-workers, developed a PBPK model to prospectively evaluate food effects and increased gastric $\mathrm{pH}$ with esomeprazole. The model predicted a positive food effect and a lack of impact of co-administration of esomeprazole. This was later confirmed in clinical trials, although the magnitude of the food effect was not accurately predicted, potentially due to excipient effects. The absorption model was further refined following confirmatory clinical studies and used to inform dose recommendations on timing of alectinib administration in relation to food intake. Authors stated that these finds were used to inform drug labeling (38).

\section{Docetaxel - pediatric dose bridging}

Docetaxel (taxane anticancer drug) is extensively metabolized by CYP3A4, substrate to the efflux transporter P-glycoprotein (P-gp), and active hepatic uptake transporters 
OATP1B1 and OATP1B3. The drug exhibits dose limiting toxicity in the form of neutropenia. In a retrospective study, a full PBPK model was developed based on adult data and validated against adult data in presence of ketoconazole. The PBPK model was then scaled to pediatrics in order to establish first dose in children assuming a similar exposure-response to adults with the same indication. A global approach was used where PBPK predictions were fitted using a pop-PK model in order to carry out optimization of sampling times. PBPK predictions of pediatric data gave a reasonable prediction with a 1.4-fold overprediction of CL (37). The study shows proof-of-concept for dose bridging from adults to pediatrics in oncology using PBPK M\&S coupled with pop-PK, to inform first-dose-in-children and optimal sampling design.

\section{Impact of physiology of oncology patients on exposure of anticancer drugs}

A number of physiological changes have been reported in cancer patients that may impact the pharmacokinetics of anticancer drugs (such as increased levels of inflammation and altered levels of plasma proteins) (56). With sufficient information, PBPK M\&S can facilitate extrapolation of drug exposure to a more clinically relevant oncology population. Cheeti, and co-workers, developed an oncology PBPK model by altering sex, age, height and weight population distributions, levels of drug-binding plasma proteins (albumin and AAG), and hematocrit to investigate the effect of plasma protein binding on exposure of midazolam (CYP3A probe) and saquinavir (CYP3A probe highly bound to AAG) (57). A similar PBPK model for oncology patients developed by Thai, and co-workers, was demonstrated to better recover variability in PK profiles and CL of docetaxel compared to when physiology was assumed to remain 
the same as in healthy (37). In the absence of physiological data, PBPK modeling can also be used to make inferences about physiological parameters based on clinical PK data. Yoshida, and co-workers, developed a PBPK of irinotecan (topoisomerase I inhibitor) and its metabolites to explore different PK of the drug in cancer patients. Using parameter estimation the authors could get an indication of the feasible parameter space of irinotecan's CL pathways in cancer patients (58).

\section{Pharmacokinetic modeling of anticancer drugs in healthcare}

Lately, there has been much debate how PK modeling can be used to aid precision dosing in clinical practice $(10,11,59)$. In an earlier State of the Art paper, we proposed a categorization to describe implementation of MIPD in healthcare based on current practices, these were: real-time implementation in healthcare systems, mechanistic modeling and extrapolation and model-derived dose banding (10).

Real-time implementation in healthcare systems refers to direct implementation of M\&S in-line with healthcare, e.g., software tools and integration into electronic health records (EHR). The approach is particularly well-suited for treatments where continuous measurement, such as TDM, is carried out routinely throughout the therapy. Bayesian modeling approaches are particularly well-suited for this approach where feedbackcontrol can be used to update prior parameter estimates and refine individual patient predictions as more data becomes available $(10,11)$. Mechanistic or PBPK models are a powerful tool for allowing extrapolation to for example DDIs or special populations. 
Despite many examples of application to address dosing in special populations (60), there are few examples of the approach being evaluated in clinical practice (61). This may partly be due to PBPK's reliance on drug-specific and physiological information and its inferior ability to describe IIV compared to pop-PK. This may however change over the coming years, the generation of new proteomic data (62), emergence of 'middle-out' modeling (14), and Bayesian PBPK M\&S (63) certainly makes PBPK an increasingly viable approach in precision dosing. Model-derived dose banding refers to the use PK models to develop dosing strategies based on clinically relevant covariates identified during the data analysis. This is perhaps the most practical approach although it potentially may offer less scope for dose individualization compared to other modelbased approaches (64-66). Based on previous experiences, work streams have been proposed for how to develop these model-based approaches from conception to implementation in clinical practice. The proposed necessary steps to prove clinical effectiveness of MIPD include: model development, internal validation - to diagnose model misspecifications, external validation - to test performance against a different but related population sample, prospective clinical evaluation - to test the performance of the model-informed approach compared to standard practice, and an implementation phase - for integration into clinical practice $(10,67,68)$. Here follows examples of PK modeling applied to answer clinically relevant dosing issues in oncology (in addition, See Table 1). These illustrate both utility and concepts for clinical evaluation and practical implementation in a healthcare.

\section{Case examples}




\section{5-fluorouracil-metabolic phenotyping}

Due to the risk of severe toxicity and a relatively narrow therapeutic index, prediction of toxicity has been widely sought for treatment using thymidylate synthase inhibitor 5fluorouracil (See Table A1, Supplementary Appendix). The drug undergoes metabolism by dihydropyrimidine dehydrogenase (DPD), where DPD deficiency has been linked to an increased risk for toxicity (69). In a retrospective study, van Kuilenburg, and coworkers, developed a Bayesian pop-PK model, based on a population sample of DPD deficient patients and controls. The model described 5FU CL using nonlinear MichaelisMenten PK, where DPD deficient patients displayed a 40\% reduction in $\mathrm{V}_{\max }$ (maximum velocity). Using a limited sampling strategy the DPD phenotype could be determined based on Bayesian estimation of individual $\mathrm{V}_{\max }$, $\mathrm{AUC}$ or terminal half-life to anticipate the risk of toxicity (70). The approach potentially offers advantages to genotyping where misclassification can occur due to discrepancies in genotype and phenotype.

\section{Carboplatin - Bayesian forecasting of drug exposure}

Despite being safer than cisplatin, carboplatin displays dose-limiting bone marrow toxicity. Duffull, and co-workers, developed a bioanalytical method for measuring carboplatin serum concentrations and a Bayesian pop-PK model with covariates lean bodyweight (LBW) and creatinine clearance on CL, and LBW on volumes and intercompartmental clearance based on data from 12 ovarian cancer patients (71). The model was tested prospectively against the Calvert et al. formula in an additional 12 patients over two courses. During the first treatment course the Calvert formula produced less bias compared to the population methods. Following two feedback concentrations the 
Bayesian method showed superior accuracy for AUC predictions. It was recognized that an updated model may produce better accuracy (72). This was perhaps one of the earliest examples incorporating patient data to update individual priors for prospective predictions $(71,72)$.

\section{Busulfan - strategies for healthcare implementation}

Busulfan (alkylating agent) is widely used in combination with cyclophosphamide for conditioning prior to hematopoietic stem cell transplantation. The narrow therapeutic window of busulfan warrants TDM in pediatrics, where up to nine PK samples are taken over the course of a single dose to inform dose adjustments. Hence numerous PK models have been published to address dose individualization of busulfan (See Table A1, Supplementary Appendix).

Neely, and co-workers, developed a nonparametric pop-PK model with age (described using a continuous polynomial function), and ideal body weight as covariates on CL and $\mathrm{V}_{\mathrm{d}}$ based on a population sample of 53 pediatric patients. An additional two datasets consisting of a total of 116 pediatric patients were then used for model validation and Bayesian updating of priors. The final model was then incorporated in to the BestDose $^{\mathrm{TM}}$ software platform and blindly tested against an additional 20 patients. The final model allowed target concentration achievement with only two blood samples per adjustment (73). 
Abdel-Rahman, and co-workers, carried out a retrospective evaluation to examine the performance of five different TDM approaches (including non-compartmental and compartmental modeling) to estimate the dose of busulfan in pediatric cancer patients and found considerable discrepancies in dose recommendations (74). Due to observed inefficiencies in the workflow for TDM guided dosing at Children's Mercy Hospital (Kansas City, MO), a clinician-oriented interface was developed around a compartmental model for dose optimization of busulfan based on TDM. The software allowed the use of either a one- or two-compartment model based on a series of quantitative goodness-of-fit criteria implemented within the software. The interface could be accessed through the EHR and was subject to usability testing by healthcare professionals. The research represents a significant step towards bringing MIPD into clinical practice and serves as a proof-of-concept for practical implementation. The authors noted that next steps will focus on quality assurance, predictive performance of the software tool and investigations of model-refinement, including inclusion of covariate effects $(75)$.

Similarly, Long-Boyle, and co-workers, developed a pop-PK model for busulfan based on retrospective data 90 pediatric and young adult patients. The final model (CL covariates: actual bodyweight and age) was then implemented in a user-friendly Microsoft Excel-based tool for guiding initial dosing in clinical practice and prospectively evaluated in 21 children. The healthcare tool showed significant improvement in attaining busulfan target concentrations compared to conventional dosing guidelines (76). 
As previously mentioned, healthcare implementation of model-based precision dosing may take on different forms; where the previous examples detailed the process of incorporating real-time software tools in healthcare. A more pragmatic approach is the derivation of dose banding based on model optimized dosing regimens. Bartelink, and co-workers, developed a two-compartment pop-PK model for busulfan with body weight as a covariate on CL based on 245 pediatric patients. The model was then used to derive a nomogram for dosing busulfan in clinical practice. The model was externally validated against an additional 158 adult and pediatric patients $(64,77)$. A prospective study was carried out to assess the performance of the model-derived nomogram and the added value of TDM was carried out in 50 pediatric patients undergoing hematopoietic stem cell transplantation. The study concluded that following the model-derived dosing variability was still significant and therefore TDM was still needed to inform dose optimization (78).

\section{Cyclophosphamide - reduced toxicity and healthcare implementation}

Cyclophosphamide (alkylating agent) is given in two doses over two consecutive days as myeloablative preparative treatment before hematopoietic stem cell transplantation. The drug is metabolized to carboxyethylphosphoramide mustard (CEPM) amongst others, where the CEPM is linked to liver toxicity and nonrelapse death. A Bayesian pop-PK model was developed for cyclophosphamide and its metabolites. The model was internally validated and incorporated into an open-source code to allow real-time dose adjustments between the two doses. This was a considerable logistical effort 
considering the time constraint for bioanalysis and model-derived dose recommendation. A clinical trial was carried out to test the performance of the modelbased dosing approach. The approach led to an average total dose reduction of around $9 \%$ and a reduction in acute liver and kidney injury with similar overall survival (7983). This case illustrates some of the logistic challenges of real-time implementation of MIPD in clinical practice.

\section{Methotrexate - reduced toxicity and healthcare implementation}

Methotrexate (antimetabolite) is used in the treatment of a number of cancers. Due to high IIV in exposure and the risk of toxicity in high-dose methotrexate treatments the drug is routinely subject to TDM. Barrett, and co-workers, developed a pop-PK model on TDM data from 240 patients at the Children's Hospital of Philadelphia (Philadelphia, PA), accounting for impaired clearance by estimating the probability of a patient belonging to one of the two subgroups. A software dashboard was developed, consisting of a database of patient records, lab data and adverse events management system. The data was then used for Bayesian forecasting of exposure. A user-interface was designed that allowed viewing of TDM data, forecasting of exposure, the potential risk of toxicity and dose guidance (84). A retrospective study was carried out in 50 pediatric and young adult patients to test the ability of the dashboard to predict future toxicity events to allow earlier recommendations of leucovorin rescue therapy. The study concluded that the dashboard gave reasonably accurate predictions (precision of $12.9 \%$, bias of $2.2 \%$ ) and could have been used to initiate earlier rescue therapy in 16 of the studied patients, seven patients would have received a larger dose of leucovorin and 37 patients would 
have received the drug less often. The dashboard can support clinicians in monitoring for risk of toxicity and guide decision making of initiation of rescue therapy (85).

\section{Pembrolizumab - minimizing excess drug wastage}

Infused anticancer drugs are sold in vials with a set volume of the drug; however dosing is often based on body size. This leads to excess drug volumes after dosing a patient which many times are being discarded at the cost of healthcare providers or insurers. It is estimated that the total cost incurred by wastage of the top 20 anticancer drugs amounted to USD 1.8bn in 2016 in the US alone (86). Pembrolizumab (programmed cell death protein 1 ligand antibody) is currently available as $50 \mathrm{mg}$ vial size in the UK at a licensed dose of $2 \mathrm{mg} / \mathrm{kg}$ every three weeks. Ogungbenro, and co-workers, proposed a model-based approach to optimize dose banding to maximize target attainment and minimize wastage. Cost analysis showed that the model-derived dosing strategy could save $16 \%$ of the cost of drug treatment compared to dosing by bodyweight by reducing discarded excess volumes of the drug, without altering exposure significantly (66). The work provides an example of how modeling can improve cost-benefit of anticancer treatment.

\section{Model-informed precision dosing in oncology: future challenges}

It has now been almost 50 years since the first model-based dosing strategies for dose individualization were proposed $(98,99)$. PK modeling has come a long way in supporting dose selection and answering clinically relevant questions in oncology and other disease areas $(10,11,59)$. While academic groups, in collaboration with 
healthcare professionals, are leading the way for model-based approaches to answer bedside dose individualization, pharmaceutical industry and regulatory agencies have made great strides in model-informed dose guidance in the drug label. It is thought that modeling will gain wider application in clinical practice over the coming years as precision medicine realizes its potential. For this to happen there are a number of challenges that need to be met.

At the moment, there is a lack of clinical effectiveness, patient benefit and health economic evidence to support MIPD in healthcare. This is crucial for wider acceptance of MIPD in clinical care. Too many published modeling efforts are concluded following model development, indicating areas of clinical application. Without rigorous validation and clinical evaluation these models will not see their full utility. Better coordination between academia, industry, healthcare, patient groups, and funding bodies are warranted to support implementation-based research in healthcare.

A prerequisite for precision dosing is the availability of multiple drug dose formulations. For MIPD to gain greater traction some adjustments to pharmaceutical R\&D would be required, with focus earlier in development of precision dosing. Recently, we illustrated how this could work using the 'companion tool' approach (10), where a MIPD tool can be considered following candidate selection and developed alongside the drug. Precision dosing can facilitate the advancement of candidate drug that otherwise would be abandoned and there is therefore, in our opinion, great financial incentives to pursue this approach in pharmaceutical industry. 
Oncology is an area with a lot to gain from PK modeling based dose individualization. There are however some specific challenges, such as the lack of exposure-effect/toxicity relationship for many new drugs coming to the market. This is of course a prerequisite for PK modeling to be meaningful. The concentration-effect relationship for monoclonal antibodies is poorly understood, where for many of these the dose-efficacy relationship found in clinical trials is flat $(100,101)$. This suggests that current dosing of monoclonal antibodies may not be optimal from an efficacy-cost perspective. Here lies an optimization challenge that can reap financial benefits for healthcare providers and payers, and in the end aid pharmaceutical industry to improve cost-benefit.

\section{Conclusions}

Here we present the current state of pharmacokinetic modeling in precision dosing of anticancer drugs. We have illustrated, using published case examples, some of the potential benefits the approach may bring in terms of prospective dosage guidance for DDIs and in special populations, improved attainment of target drug concentration and reduced risk of toxicity, reducing wastage and scope for improved patient benefit and cost-benefit. While some great progress has been seen in implementation of modelinformed dosage guidance in the drug labeling, a collaborative effort from regulators, industry and academia, the uptake has been modest in healthcare. Collaboration between academia, healthcare and industry together with greater financial support for applied research into patient benefit, cost-benefit and clinical effectiveness of modelbased dosing approaches is warranted for wider adaption in healthcare. 


\section{Acknowledgements}

Authors declare no conflict of interest.

\section{References}

1. Orloff J, Douglas F, Pinheiro J, Levinson S, Branson M, Chaturvedi P, et al. A GUIDE TO DRUG DISCOVERY - OPINION The future of drug development: advancing clinical trial design. Nature Reviews Drug Discovery. 2009;8(12):949-57.

2. Felici A, Verweij J, Sparreboom A. Dosing strategies for anticancer drugs: the good, the bad and body-surface area. Eur J Cancer. 2002;38(13):1677-84. Epub 2002/08/15.

3. Pinkel D. The use of body surface area as a criterion of drug dosage in cancer chemotherapy. Cancer Res. 1958;18(7):853-6. Epub 1958/08/01.

4. Beumer JH, Chu E, Salamone SJ. Body-surface area-based chemotherapy dosing: appropriate in the 21st century? J Clin Oncol. 2012;30(31):3896-7. Epub 2012/09/12.

5. Baker SD, Verweij J, Rowinsky EK, Donehower RC, Schellens JHM, Grochow LB, et al. Role of body surface area in dosing of investigational anticancer agents in adults, 1991-2001. Journal of the National Cancer Institute. 2002;94(24):1883-8.

6. Grimsrud KN, Sherwin CM, Constance JE, Tak C, Zuppa AF, Spigarelli MG, et al. Special population considerations and regulatory affairs for clinical research. Clinical research and regulatory affairs. 2015;32(2):47-56. Epub 2015/09/25.

7. Jadhav PR, Cook J, Sinha V, Zhao P, Rostami-Hodjegan A, Sahasrabudhe V, et al. A proposal for scientific framework enabling specific population drug dosing recommendations. J Clin Pharmacol. 2015;55(10):1073-8. Epub 2015/06/26.

8. van der Elst KCM, Pereboom M, van den Heuvel ER, Kosterink JGW, Scholvinck EH, Alffenaar JWC. Insufficient Fluconazole Exposure in Pediatric Cancer Patients and the Need for Therapeutic Drug Monitoring in Critically Ill Children. Clin Infect Dis. 2014;59(11):1527-33.

9. Launay-Vacher V, Janus N, Deray G. Renal insufficiency and cancer treatments. ESMO open. 2016;1(4):e000091. Epub 2016/11/16.

10. Darwich AS, Ogungbenro K, Vinks AA, Powell JR, Reny JL, Marsousi N, et al. Why has model-informed precision dosing not yet become common clinical reality? lessons from the past and a roadmap for the future. Clinical pharmacology and therapeutics. 2017;101(5):646-56. 11. Neely M. Scalpels not hammers: The way forward for precision drug prescription. Clin Pharmacol Ther. 2017;101(3):368-72. Epub 2016/12/17.

12. Younis IR, Powell JR, Rostami-Hodjegan A, Corrigan B, Stockbridge N, Sinha V, et al. Utility of Model Based Approaches for Informing Dosing Recommendations in Specific Populations: Report from the Public AAPS Workshop. J Clin Pharmacol. 2016. Epub 2016/07/02.

13. Wong KM, Capasso A, Eckhardt SG. The changing landscape of phase I trials in oncology. Nature reviews Clinical oncology. 2016;13(2):106-17. Epub 2015/11/11.

14. Tsamandouras N, Rostami-Hodjegan A, Aarons L. Combining the "bottom-up" and "top-down" approaches in pharmacokinetic modelling: Fitting PBPK models to observed clinical data. Br J Clin Pharmacol. 2013. 
15. Jamei M. Recent Advances in Development and Application of Physiologically-Based Pharmacokinetic (PBPK) Models: a Transition from Academic Curiosity to Regulatory Acceptance. Current pharmacology reports. 2016;2:161-9. Epub 2016/05/27.

16. Collins FS, Varmus H. A new initiative on precision medicine. The New England journal of medicine. 2015;372(9):793-5. Epub 2015/01/31.

17. Calvert AH, Newell DR, Gumbrell LA, O'Reilly S, Burnell M, Boxall FE, et al. Carboplatin dosage: prospective evaluation of a simple formula based on renal function. J Clin Oncol. 1989;7(11):1748-56. Epub 1989/11/01.

18. Capitain O, Asevoaia A, Boisdron-Celle M, Poirier AL, Morel A, Gamelin E. Individual fluorouracil dose adjustment in FOLFOX based on pharmacokinetic follow-up compared with conventional body-area-surface dosing: a phase II, proof-of-concept study. Clinical colorectal cancer. 2012;11(4):263-7. Epub 2012/06/12.

19. Yoshida K, Budha N, Jin JY. Impact of physiologically based pharmacokinetic models on regulatory reviews and product labels: Frequent utilization in the field of oncology. Clin Pharmacol Ther. 2017;101(5):597-602. Epub 2017/01/12.

20. Hait WN. Anticancer drug development: the grand challenges. Nature Reviews Drug Discovery. 2010;9(4):253-4.

21. Siddiqui M, Rajkumar SV. The High Cost of Cancer Drugs and What We Can Do About It. Mayo Clinic proceedings. 2012;87(10):935-43.

22. Workgroup EM, Marshall SF, Burghaus R, Cosson V, Cheung SY, Chenel M, et al. Good Practices in Model-Informed Drug Discovery and Development: Practice, Application, and Documentation. CPT: pharmacometrics \& systems pharmacology. 2016;5(3):93-122. Epub 2016/04/14.

23. Mould DR, Upton RN. Basic concepts in population modeling, simulation, and modelbased drug development-part 2: introduction to pharmacokinetic modeling methods. CPT: pharmacometrics \& systems pharmacology. 2013;2:e38. Epub 2013/07/28.

24. Williams PJ, Ette EI. The role of population pharmacokinetics in drug development in light of the Food and Drug Administration's 'Guidance for Industry: Population pharmacokinetics'. Clin Pharmacokinet. 2000;39(6):385-95.

25. Joerger M. Covariate pharmacokinetic model building in oncology and its potential clinical relevance. AAPS J. 2012;14(1):119-32. Epub 2012/01/26.

26. Ribbing J, Jonsson EN. Power, selection bias and predictive performance of the Population Pharmacokinetic Covariate Model. J Pharmacokinet Pharmacodyn. 2004;31(2):10934.

27. Duffull SB, Wright DFB, Winter HR. Interpreting population pharmacokineticpharmacodynamic analyses - a clinical viewpoint. Br J Clin Pharmacol. 2011;71(6):807-14. 28. FDA. Taxotere(R) highlights of presribing information. 2015 [cited 2017 June 14]; Available from: https://www.accessdata.fda.gov/drugsatfda_docs/label/2015/020449s075lbl.pdf. 29. FDA. Avastin(R) highlights of prescribing information. 2016 [cited 2017 June 14]; Available from: https://www.accessdata.fda.gov/drugsatfda_docs/label/2016/125085s317lbl.pdf. 30. FDA. Zaltrap(R) highlights of prescribing information. 2016 [cited 2017 June 14]; Available from: https://www.accessdata.fda.gov/drugsatfda_docs/label/2016/125418s039lbl.pdf. 31. FDA. Xeloda(R) highlights of prescribing information. 2016 [cited 2017 June 14]; Available from: https://www.accessdata.fda.gov/drugsatfda_docs/label/2016/020896s039lbl.pdf. 32. FDA. Carboplatin (TEVA Pharmaceuticals USA) highlights of prescribing information. 2012 [cited 2017 June 14]; Available from:

https://www.accessdata.fda.gov/drugsatfda_docs/label/2012/077139Orig1 s016lbl.pdf. 33. FDA. Busulfex(R) highlights of prescribing information. 2016 [cited 2017 June 14]; Available from: https://www.accessdata.fda.gov/drugsatfda_docs/label/2016/020954s016lbl.pdf. 34. Chen HS, Gross JF. Physiologically based pharmacokinetic models for anticancer drugs. Cancer Chemother Pharmacol. 1979;2(2):85-94. Epub 1979/01/01. 
35. Walsh C, Bonner JJ, Johnson TN, Neuhoff S, Ghazaly EA, Gribben JG, et al. Development of a physiologically based pharmacokinetic model of actinomycin D in children with cancer. Br J Clin Pharmacol. 2016;81(5):989-98. Epub 2016/01/05.

36. Diestelhorst C, Boos J, McCune JS, Russell J, Kangarloo SB, Hempel G. Predictive performance of a physiologically based pharmacokinetic model of busulfan in children. Pediatric hematology and oncology. 2014;31(8):731-42. Epub 2014/07/10.

37. Thai HT, Mazuir F, Cartot-Cotton S, Veyrat-Follet C. Optimizing pharmacokinetic bridging studies in paediatric oncology using physiologically-based pharmacokinetic modelling: application to docetaxel. Br J Clin Pharmacol. 2015;80(3):534-47. Epub 2015/06/23. 38. Parrott NJ, Yu LJ, Takano R, Nakamura M, Morcos PN. Physiologically Based Absorption Modeling to Explore the Impact of Food and Gastric $\mathrm{pH}$ Changes on the Pharmacokinetics of Alectinib. AAPS J. 2016;18(6):1464-74. Epub 2016/11/05.

39. Tan W, Yamazaki S, Johnson TR, Wang R, O'Gorman MT, Kirkovsky L, et al. Effects of Renal Function on Crizotinib Pharmacokinetics: Dose Recommendations for Patients with ALK-Positive Non-Small Cell Lung Cancer. Clinical drug investigation. 2017;37(4):363-73. Epub 2016/12/31.

40. Fujita K, Masuo Y, Okumura H, Watanabe Y, Suzuki H, Sunakawa Y, et al. Increased Plasma Concentrations of Unbound SN-38, the Active Metabolite of Irinotecan, in Cancer Patients with Severe Renal Failure. Pharm Res. 2016;33(2):269-82. Epub 2015/09/05.

41. Lu C, Suri A, Shyu WC, Prakash S. Assessment of cytochrome P450-mediated drugdrug interaction potential of orteronel and exposure changes in patients with renal impairment using physiologically based pharmacokinetic modeling and simulation. Biopharm Drug Dispos. 2014;35(9):543-52. Epub 2014/09/30.

42. Emoto C, Fukuda T, Cox S, Christians U, Vinks AA. Development of a PhysiologicallyBased Pharmacokinetic Model for Sirolimus: Predicting Bioavailability Based on Intestinal CYP3A Content. CPT: pharmacometrics \& systems pharmacology. 2013;2:e59. Epub 2013/07/26.

43. Dickschen K, Willmann S, Thelen K, Lippert J, Hempel G, Eissing T. Physiologically Based Pharmacokinetic Modeling of Tamoxifen and its Metabolites in Women of Different CYP2D6 Phenotypes Provides New Insight into the Tamoxifen Mass Balance. Front Pharmacol. 2012;3:92. Epub 2012/06/05.

44. Dickschen KJR, Willmann S, Hempel G, Block M. Addressing Adherence Using Genotype-Specific PBPK Modeling-Impact of Drug Holidays on Tamoxifen and Endoxifen Plasma Levels. Front Pharmacol. 2017;8.

45. Jing J, Nelson C, Paik J, Shirasaka Y, Amory JK, Isoherranen N. Physiologically Based Pharmacokinetic Model of All-trans-Retinoic Acid with Application to Cancer Populations and Drug Interactions. J Pharmacol Exp Ther. 2017;361(2):246-58. Epub 2017/03/10.

46. Glenn KJ, Yu LJ, Reddy MB, Fretland AJ, Parrott N, Hussain S, et al. Investigating the effect of autoinduction in cynomolgus monkeys of a novel anticancer MDM2 antagonist, idasanutlin, and relevance to humans. Xenobiotica. 2016;46(8):667-76. Epub 2015/11/21.

47. Posada MM, Bacon JA, Schneck KB, Tirona RG, Kim RB, Higgins JW, et al. Prediction of renal transporter mediated drug-drug interactions for pemetrexed using physiologically based pharmacokinetic modeling. Drug Metab Dispos. 2015;43(3):325-34. Epub 2014/12/17. 48. Yamazaki S, Johnson TR, Smith BJ. Prediction of Drug-Drug Interactions with Crizotinib as the CYP3A Substrate Using a Physiologically Based Pharmacokinetic Model. Drug metabolism and disposition: the biological fate of chemicals. 2015;43(10):1417-29. 49. Mao J, Johnson TR, Shen Z, Yamazaki S. Prediction of crizotinib-midazolam interaction using the Simcyp population-based simulator: comparison of CYP3A timedependent inhibition between human liver microsomes versus hepatocytes. Drug metabolism and disposition: the biological fate of chemicals. 2013;41(2):343-52. Epub 2012/11/07. 50. Pharmaceutical Science and Clinical Pharmacology Advisory Committee Meeting, March 15, 2017. In: Center for Drug Evaluation and Research USFaDA, editor. 2017. 
51. National Cancer Institute NIoHN. FDA Approval for IbrutinibNovember 19, 2013 June 13, 2017. Available from: https://www.cancer.gov/about-cancer/treatment/drugs/fda-ibrutinib.

52. Fowler S, Morcos PN, Cleary Y, Martin-Facklam M, Parrott N, Gertz M, et al. Progress in Prediction and Interpretation of Clinically Relevant Metabolic Drug-Drug Interactions: a Minireview Illustrating Recent Developments and Current Opportunities. Current pharmacology reports. 2017;3(1):36-49. Epub 2017/03/07.

53. de Zwart L, Snoeys J, De Jong J, Sukbuntherng J, Mannaert E, Monshouwer M. Ibrutinib Dosing Strategies Based on Interaction Potential of CYP3A4 Perpetrators Using Physiologically Based Pharmacokinetic Modeling. Clin Pharmacol Ther. 2016;100(5):548-57. Epub 2016/07/02.

54. Einolf HJ, Zhou J, Won C, Wang L, Rebello S. A Physiologically-Based Pharmacokinetic Modeling Approach To Predict Drug-Drug Interactions of Sonidegib (LDE225) with Perpetrators of CYP3A in Cancer Patients. Drug metabolism and disposition: the biological fate of chemicals. 2017;45(4):361-74.

55. Administration USFD. FDA approves new oral therapy to treat ALK-positive lung cancer. December 11, 2015 [cited 2017 June 13]; Available from: https://www.fda.gov/newsevents/newsroom/pressannouncements/ucm476926.htm. 56. Coutant DE, Kulanthaivel P, Turner PK, Bell RL, Baldwin J, Wijayawardana SR, et al. Understanding Disease-Drug Interactions in Cancer Patients: Implications for Dosing Within the Therapeutic Window. Clin Pharmacol Ther. 2015;98(1):76-86. Epub 2015/03/27. 57. Cheeti S, Budha NR, Rajan S, Dresser MJ, Jin JY. A physiologically based pharmacokinetic (PBPK) approach to evaluate pharmacokinetics in patients with cancer. Biopharm Drug Dispos. 2013;34(3):141-54. Epub 2012/12/12.

58. Yoshida K, Maeda K, Kusuhara H, Konagaya A. Estimation of feasible solution space using Cluster Newton Method: application to pharmacokinetic analysis of irinotecan with physiologically-based pharmacokinetic models. BMC systems biology. 2013;7 Suppl 3:S3. Epub 2014/02/22.

59. Neely M, Jelliffe R. Practical, individualized dosing: 21 st century therapeutics and the clinical pharmacometrician. J Clin Pharmacol. 2010;50(7):842-7. Epub 2010/02/16.

60. Marsousi N, Desmeules JA, Rudaz S, Daali Y. Usefulness of PBPK modeling in incorporation of clinical conditions in personalized medicine. J Pharm Sci-Us. 2017.

61. Marsousi N, Samer CF, Fontana P, Reny JL, Rudaz S, Desmeules JA, et al. Coadministration of ticagrelor and ritonavir: Toward prospective dose adjustment to maintain an optimal platelet inhibition using the PBPK approach. Clin Pharmacol Ther. 2016;100(3):295304. Epub 2016/06/07.

62. Heikkinen AT, Lignet F, Cutler P, Parrott N. The role of quantitative ADME proteomics to support construction of physiologically based pharmacokinetic models for use in small molecule drug development. Proteomics Clin Appl. 2015;9(7-8):732-44.

63. Krauss M, Burghaus R, Lippert J, Niemi M, Neuvonen P, Schuppert A, et al. Using Bayesian-PBPK modeling for assessment of inter-individual variability and subgroup stratification. In silico pharmacology. 2013;1:6. Epub 2013/01/01.

64. Bartelink IH, van Kesteren C, Boelens JJ, Egberts TC, Bierings MB, Cuvelier GD, et al. Predictive performance of a busulfan pharmacokinetic model in children and young adults. Ther Drug Monit. 2012;34(5):574-83. Epub 2012/09/14.

65. Krekels EH, Tibboel D, de Wildt SN, Ceelie I, Dahan A, van Dijk M, et al. Evidencebased morphine dosing for postoperative neonates and infants. Clin Pharmacokinet. 2014;53(6):553-63. Epub 2014/02/06.

66. Ogungbenro K, Patel A, Duncombe R, Clark J, Lorigan P. A rational approach to dose optimisation of pembrolizumab using cost analysis and pharmacokinetic modelling and simulation. J Clin Oncol. 2016;34(Suppl):abstr 9547.

67. Ince I, de Wildt SN, Tibboel D, Danhof M, Knibbe CA. Tailor-made drug treatment for children: creation of an infrastructure for data-sharing and population PK-PD modeling. Drug discovery today. 2009;14(5-6):316-20. Epub 2008/12/09. 
68. Vinks AA, Derendorf H, Mouton JW. Fundamentals of antimicrobial pharmacokinetics and pharmacodynamics. New York, USA: Springer Science+Business Media; 2014. ix, 466 pages $\mathrm{p}$.

69. van Kuilenburg ABP, Haasjes J, Richel DJ, Zoetekouw L, Van Lenthe H, De Abreu RA, et al. Clinical implications of dihydropyrimidine dehydrogenase (DPD) deficiency in patients with severe 5-fluorouracil-associated toxicity: Identification of new mutations in the DPD gene. Clinical cancer research : an official journal of the American Association for Cancer Research. 2000;6(12):4705-12.

70. van Kuilenburg ABP, Hausler P, Schalhorn A, Tanck MWT, Proost JH, Terborg C, et al. Evaluation of 5-Fluorouracil Pharmacokinetics in Cancer Patients with a c.1905+1G > A Mutation in DPYD by Means of a Bayesian Limited Sampling Strategy. Clin Pharmacokinet. 2012;51(3):163-74.

71. Duffull SB, Begg EJ, Robinson BA, Deely JJ. A sequential Bayesian algorithm for dose individualisation of carboplatin. Cancer Chemother Pharmacol. 1997;39(4):317-26. Epub 1997/01/01.

72. Duffull SB, Robinson BA. Clinical pharmacokinetics and dose optimisation of carboplatin. Clin Pharmacokinet. 1997;33(3):161-83. Epub 1997/10/07.

73. Neely M, Philippe M, Rushing T, Fu X, van Guilder M, Bayard D, et al. Accurately Achieving Target Busulfan Exposure in Children and Adolescents With Very Limited Sampling and the BestDose Software. Ther Drug Monit. 2016;38(3):332-42. Epub 2016/02/02. 74. Abdel-Rahman SM, Casey KL, Garg U, Dalal J. Intravenous busulfan dose individualization - impact of modeling approach on dose recommendation. Pediatric transplantation. 2016;20(3):443-8. Epub 2016/02/09.

75. Abdel-Rahman SM, Breitkreutz ML, Bi C, Matzuka BJ, Dalal J, Casey KL, et al. Design and Testing of an EHR-Integrated, Busulfan Pharmacokinetic Decision Support Tool for the Point-of-Care Clinician. Front Pharmacol. 2016;7:65. Epub 2016/04/12.

76. Long-Boyle JR, Savic R, Yan S, Bartelink I, Musick L, French D, et al. Population Pharmacokinetics of Busulfan in Pediatric and Young Adult Patients Undergoing Hematopoietic Cell Transplant: A Model-Based Dosing Algorithm for Personalized Therapy and Implementation Into Routine Clinical Use. Ther Drug Monit. 2015;37(2):236-45.

77. Bartelink IH, Boelens JJ, Bredius RG, Egberts AC, Wang C, Bierings MB, et al. Body weight-dependent pharmacokinetics of busulfan in paediatric haematopoietic stem cell transplantation patients: towards individualized dosing. Clin Pharmacokinet. 2012;51(5):33145. Epub 2012/03/30.

78. van Reij E, van Maarseveen E, Bartelink I, Rademaker C, Egberts T, Boelens JJ. Prospective Validation of a Busulfan Pharmacokinetic Model in Children: Therapeutic Drug Monitoring Remains of Utmost Importance to Optimize Outcomes of HCT. Biol Blood Marrow Tr. 2014;20(2):S248-S.

79. McDonald GB, Slattery JT, Bouvier ME, Ren S, Batchelder AL, Kalhorn TF, et al. Cyclophosphamide metabolism, liver toxicity, and mortality following hematopoietic stem cell transplantation. Blood. 2003;101(5):2043-8. Epub 2002/10/31.

80. McDonald GB, McCune JS, Batchelder A, Cole S, Phillips B, Ren AG, et al. Metabolism-based cyclophosphamide dosing for hematopoietic cell transplant. Clin Pharmacol Ther. 2005;78(3):298-308. Epub 2005/09/13.

81. McCune JS, Batchelder A, Guthrie KA, Witherspoon R, Appelbaum FR, Phillips B, et al. Personalized dosing of cyclophosphamide in the total body irradiation-cyclophosphamide conditioning regimen: a phase II trial in patients with hematologic malignancy. Clin Pharmacol Ther. 2009;85(6):615-22. Epub 2009/03/20.

82. Salinger DH, McCune JS, Ren AG, Shen DD, Slattery JT, Phillips B, et al. Real-time dose adjustment of cyclophosphamide in a preparative regimen for hematopoietic cell transplant: a Bayesian pharmacokinetic approach. Clin Cancer Res. 2006;12(16):4888-98. Epub 2006/08/18. 
83. Qiu R, Yao A, Vicini P, McDonald GB, Batchelder AL, Bouvier ME, et al. Diminishing the risk of nonrelapse mortality in hematopoietic stem cell transplantation: Prediction of exposure to the cyclophosphamide metabolite carboxyethylphosphoramide mustard. Clin Pharmacol Ther. 2004;76(3):270-80. Epub 2004/09/17.

84. Barrett JS, Mondick JT, Narayan M, Vijayakumar K, Vijayakumar S. Integration of modeling and simulation into hospital-based decision support systems guiding pediatric pharmacotherapy. BMC medical informatics and decision making. 2008;8:6. Epub 2008/01/30. 85. Dombrowsky E, Jayaraman B, Narayan M, Barrett JS. Evaluating performance of a decision support system to improve methotrexate pharmacotherapy in children and young adults with cancer. Ther Drug Monit. 2011;33(1):99-107. Epub 2010/12/31.

86. Bach PB, Conti RM, Muller RJ, Schnorr GC, Saltz LB. Overspending driven by oversized single dose vials of cancer drugs. Bmj. 2016;352:i788. Epub 2016/03/05.

87. Bartelink IH, Lalmohamed A, van Reij EM, Dvorak CC, Savic RM, Zwaveling J, et al. Busulfan Exposure Predicts Event Free Survival and Toxicity after Hematopoietic Cell Transplantation in Children And Young Adults: A Multicenter Retrospective Cohort Analysis. Bone marrow transplantation. 2016;51:S14-S.

88. Peng B, Boddy AV, Cole M, Pearson AD, Chatelut E, Rubie H, et al. Comparison of methods for the estimation of carboplatin pharmacokinetics in paediatric cancer patients. Eur J Cancer. 1995;31A(11):1804-10. Epub 1995/10/01.

89. Chatelut E, Pivot X, Otto J, Chevreau C, Thyss A, Renee N, et al. A limited sampling strategy for determining carboplatin AUC and monitoring drug dosage. Eur J Cancer. 2000;36(2):264-9.

90. McCune JS, Salinger DH, Vicini P, Oglesby C, Blough DK, Park JR. Population Pharmacokinetics of Cyclophosphamide and Metabolites in Children With Neuroblastoma: A Report From the Children's Oncology Group. J Clin Pharmacol. 2009;49(1):88-102.

91. Vainas O, Ariad S, Amir O, Mermershtain W, Vainstein V, Kleiman M, et al. Personalising docetaxel and G-CSF schedules in cancer patients by a clinically validated computational model. Br J Cancer. 2012;107(5):814-22. Epub 2012/07/21.

92. Monjanel-Mouterde S, Lejeune C, Ciccolini J, Merite N, Hadjaj D, Bonnier P, et al. Bayesian population model of methotrexate to guide dosage adjustments for folate rescue in patients with breast cancer. J Clin Pharm Ther. 2002;27(3):189-95. Epub 2002/06/26.

93. Pignon T, Lacarelle B, Duffaud F, Guillet P, Catalin J, Durand A, et al. Pharmacokinetics of high-dose methotrexate in adult osteogenic sarcoma. Cancer Chemother Pharmacol. 1994;33(5):420-4. Epub 1994/01/01.

94. Pignon T, Lacarelle B, Duffaud F, Guillet P, Catalin J, Durand A, et al. Dosage Adjustment of High-Dose Methotrexate Using Bayesian-Estimation - a Comparative-Study of 2 Different Concentrations at the End of 8-H Infusions. Ther Drug Monit. 1995;17(5):471-8. 95. Ahamadi M, Freshwater T, Prohn M, Li CH, de Alwis DP, de Greef R, et al. ModelBased Characterization of the Pharmacokinetics of Pembrolizumab: A Humanized Anti-PD-1 Monoclonal Antibody in Advanced Solid Tumors. CPT: pharmacometrics \& systems pharmacology. 2017;6(1):49-57. Epub 2016/11/20.

96. van Rijswijk REN, Vanloenen AC, Wagstaff J, Meijer E, Lopez R, Vangroeningen CJ, et al. Suramin - Rapid Loading and Weekly Maintenance Regimens for Cancer-Patients. Journal of clinical oncology : official journal of the American Society of Clinical Oncology.

1992;10(11):1788-94.

97. Jodrell DI, Reyno LM, Sridhara R, Eisenberger MA, Tkaczuk KH, Zuhowski EG, et al. Suramin - Development of a Population Pharmacokinetic Model and Its Use with Intermittent Short Infusions to Control Plasma Drug Concentration in Patients with Prostate-Cancer. Journal of clinical oncology : official journal of the American Society of Clinical Oncology. 1994;12(1):166-75.

98. Sheiner LB. Computer-aided long-term anticoagulation therapy. Computers and biomedical research, an international journal. 1969;2(6):507-18. Epub 1969/12/01. 
99. Jelliffe RW. Administration of digoxin. Diseases of the chest. 1969;56(1):56-60. Epub $1969 / 07 / 01$.

100. research Cfdea. Clinical pharmacology and biopharmaceutics reviews of nivolumab: application number: 125554Orig1s000. 2015.

101. research Cfdea. Clinical pharmacology and biopharmaceutics reviews of pembrolizumab: application number: 125514Orig1s000. 2014 [June 13, 2017]; Available from: http://www.accessdata.fda.gov/drugsatfda_docs/nda/2014/125514Orig1s000ClinPharmR.pdf

Figure 1. Pharmacokinetic modeling approaches for precision dosing of oncology drugs. Bars indicate the features of the individual and combined techniques.

Figure 2. Peer-reviewed publications on pharmacokinetic modeling of oncology drugs categorized based on method of approach ( $\mathrm{N}$ publications: 393, $\mathrm{N}$ drug-publication combinations: 414, See Supplementary Appendix). PK: pharmacokinetic, PD: pharmacodynamic, pop: population-based analysis, semi-mech.: semi-mechanistic/semiphysiological, PBPK: physiologically-based pharmacokinetics.

Figure 3. Areas of application of pharmacokinetic modeling in oncology based on a sample of 393 peer-reviewed publications (See Supplementary Appendix). Circle areas are proportional to frequencies. DDIs: drug-drug interactions.

Figure 4. Identified pharmacokinetic covariates of oncology drugs based on a literature sample set of 393 peer-reviewed publications, see Supplementary Appendix. Numerical prefixes/suffixes show number of identified covariates for respective drug (and their metabolites where applicable), percentage suffixes show frequencies of drugs that 
identify respective covariate. AAG: $\alpha_{1}$-acid glycoprotein, ALK: alkaline phosphatase, ALT: alanine amino transferase, AST: aspartate amino transferase, BSA: body surface area, BW: bodyweight, IBW: ideal bodyweight, $\mathrm{CL}_{\mathrm{CR}}$ : creatinine clearance, DDI: drugdrug interaction, EGF: epidermal growth factor, EGFR: EGF receptor, erythromycin breath test: as surrogate of CYP3A4 activity, GFR/eGFR: estimated glomerular filtration rate, HER: human epidermal growth factor receptor, IFN- $\alpha$ : interferon- $\alpha$, LBW: lean bodyweight, LD: lactate dehydrogenase, PDGF: platelet-derived growth factor, $\mathrm{S}_{\mathrm{CR}}$ : serum creatinine, VEGF: vascular endothelial growth factor.

Figure 5. Factors affecting explicit dose recommendations in the FDA drug labels (including CI: contraindications) of docetaxel $\left(\right.$ Taxotere $^{\circledR}$ ), bevacizumab (Avastin ${ }^{\circledR}$ ), aflibercept $\left(\right.$ Zaltrap $\left.^{\circledR}\right)$, capecitabine $\left(\right.$ Xeloda $\left.^{\circledR}\right)$, carboplatin (TEVA Pharmaceuticals USA) and busulfan (Busulfex ${ }^{\circledR}$; blue circles) (28-33). Grey circles indicate additional pharmacokinetic covariates identified in the sampled peer-reviewed literature (see Table A1, Supplementary Appendix). Exposed circle areas are proportional to the number of factors/covariates. AAG: $\alpha_{1}$-acid glycoprotein, AIBW: adjusted IBW, BW: bodyweight, BSA: body surface area, $\mathrm{CL}_{\mathrm{CR}}$ : creatinine clearance, DDIs: drug-drug interactions, erythromycin breath test: as surrogate of CYP3A4 activity, IBW: ideal bodyweight, IFN- $\alpha$ : interferon- $\alpha$, liver function: including AST, ALT and more, excluding albumin. 


\begin{tabular}{|c|c|c|c|c|c|c|}
\hline Drug & $\begin{array}{c}\text { Aim of } \\
\text { modeling }\end{array}$ & $\begin{array}{c}\text { PK } \\
\text { modeling } \\
\text { approach }\end{array}$ & $\begin{array}{l}\text { Identified } \\
\text { covariates }\end{array}$ & $\begin{array}{c}\text { Study } \\
\text { approach } \\
\text { (N patients) }\end{array}$ & Outcomes & References \\
\hline $\begin{array}{l}\text { 5-fluorouracil } \\
\text { (5FU) }\end{array}$ & $\begin{array}{l}\text { Develop limited } \\
\text { sampling to } \\
\text { detect PDP- } \\
\text { deficiency. }\end{array}$ & $\begin{array}{l}\text { Pop-PK: 2- } \\
\text { cmp }\left(V_{\max }\right. \\
\left.\mathrm{K}_{\mathrm{m}}\right)^{\mathrm{A}}\end{array}$ & $\begin{array}{l}\text { Significant } \\
\text { difference in } \\
V_{\max } \text { between } \\
D P D- \\
\text { deficient and } \\
\text { controls } \\
\end{array}$ & $\begin{array}{l}\text { Development } \\
(\mathrm{N}=48) ; \\
\text { validation } 1 \\
(\mathrm{~N}=33)\end{array}$ & $\begin{array}{l}\text { Limited sampling } \\
\text { schedule to identify } \\
\text { PDP-deficient } \\
\text { patients at risk of } \\
\text { toxicity. }\end{array}$ & (70) \\
\hline \multirow{5}{*}{ busulfan } & $\begin{array}{l}\text { Develop and } \\
\text { implement PK } \\
\text { model to achieve } \\
\text { target exposure } \\
\text { in HCT children. }\end{array}$ & $\begin{array}{l}\text { Bayesian } \\
\text { pop-PK: 1- } \\
\text { cmp }(C L)^{\mathrm{A}}\end{array}$ & CL: BW, Age & $\begin{array}{l}\text { Development } \\
(\mathrm{N}=90) ; \\
\text { prospective } \\
\text { validation } 1 \\
(\mathrm{~N}=21)\end{array}$ & $\begin{array}{l}\text { Model-based } \\
\text { algorithm improved } \\
\text { target achievement } \\
\text { compared to } \\
\text { conventional } \\
\text { dosing. }\end{array}$ & (76) \\
\hline & $\begin{array}{l}\text { Examine five } \\
\text { different TDM } \\
\text { based dosing } \\
\text { approaches and } \\
\text { implement in } \\
\text { EHR. }\end{array}$ & PK: $1 / 2$-cmp & $\mathrm{N} / \mathrm{A}$ & $\begin{array}{l}\text { Development/ } \\
\text { validation: } \\
(\mathrm{N}=30) ; \\
\text { software } \\
\text { usability } \\
\text { testing }\end{array}$ & $\begin{array}{l}\text { Software tool for } \\
\text { interpreting TDM } \\
\text { was implemented in } \\
\text { EHR. }\end{array}$ & $(74,75)$ \\
\hline & $\begin{array}{l}\text { Pediatric dose } \\
\text { adjustment based } \\
\text { on limited } \\
\text { sampling. }\end{array}$ & $\begin{array}{l}\text { Bayesian } \\
\text { pop-PK: } 1- \\
\mathrm{cmp}\left(\mathrm{k}_{\mathrm{el}}\right)^{\mathrm{A}}\end{array}$ & Age, IBW & $\begin{array}{l}\text { Development } \\
(\mathrm{N}=53) ; \\
\text { validation } 1 \\
(\mathrm{~N}=105) ; \\
\text { validation } 2 \\
(\mathrm{~N}=11) ; \\
\text { retrospective } \\
\text { clinical } \\
\text { evaluation } \\
(\mathrm{N}=20)\end{array}$ & $\begin{array}{l}\text { Model allowed } \\
\text { limited sampling to } \\
\text { achieve target } \\
\text { concentrations. }\end{array}$ & (73) \\
\hline & $\begin{array}{l}\text { Explore } \\
\text { variability in PK } \\
\text { in children and } \\
\text { young adults to } \\
\text { optimize dosing. }\end{array}$ & $\begin{array}{l}\text { Pop-PK: 2- } \\
\text { cmp }(C L)^{A}\end{array}$ & $\begin{array}{l}\text { CL: BW, } \\
\text { Day; } \\
\mathrm{V}_{1}: \text { BW }\end{array}$ & $\begin{array}{l}\text { Development } \\
(\mathrm{N}=245) ; \\
\text { validation } 1 \\
(\mathrm{~N}=158) ; \\
\text { prospective } \\
\text { clinical } \\
\text { evaluation } \\
(\mathrm{N}=50)\end{array}$ & $\begin{array}{l}\text { Nomogram based } \\
\text { on PK model. } \\
\text { Dosing scheme led } \\
\text { to some residual } \\
\text { variability, needing } \\
\text { TDM. }\end{array}$ & $\begin{array}{l}(64,77, \\
78)\end{array}$ \\
\hline & $\begin{array}{l}\text { Evaluate } \\
\text { approaches to } \\
\text { estimate } \\
\text { exposure and } \\
\text { clinical outcome } \\
\text { in children and } \\
\text { young adults. }\end{array}$ & $\begin{array}{l}\text { Pop-PK } \\
\text { (refitted) } \\
\text { (77) }\end{array}$ & N/A & $\begin{array}{l}\text { Evaluation } \\
(\mathrm{N}=674)\end{array}$ & $\begin{array}{l}\text { The approach may } \\
\text { help optimize target } \\
\text { exposure and } \\
\text { improve } \\
\text { efficacy/toxicity. }\end{array}$ & (87) \\
\hline \multirow[t]{3}{*}{ carboplatin } & $\begin{array}{l}\text { Develop limited } \\
\text { sampling } \\
\text { strategy. }\end{array}$ & $\begin{array}{l}\text { Bayesian } \\
\text { pop-PK: } 1- \\
\mathrm{cmp}\left(\mathrm{k}_{\mathrm{el}}\right)^{\mathrm{A}}\end{array}$ & $\mathrm{N} / \mathrm{A}$ & $\begin{array}{l}\text { Development } \\
(\mathrm{N}=22) ; \\
\text { validation } 1 \\
(\mathrm{~N}=15)\end{array}$ & $\begin{array}{l}\text { Reduced bias and } \\
\text { increased precision } \\
\text { for AUC estimation } \\
\text { using one or two } \\
\text { samples. }\end{array}$ & (88) \\
\hline & $\begin{array}{l}\text { Develop } \\
\text { Bayesian dose } \\
\text { adjustment }\end{array}$ & $\begin{array}{l}\text { Bayesian } \\
\text { pop-PK: } \\
2 \mathrm{cmp}(\mathrm{CL})^{\mathrm{A}}\end{array}$ & $\begin{array}{l}\text { CL: LBW, } \\
\text { CL }_{\mathrm{CR}} ; \\
\mathrm{V}_{\mathrm{ss}}: \mathrm{LBW} ; \\
\mathrm{V}_{1}: \mathrm{LBW} ; \\
\mathrm{CL}_{\mathrm{ic}}: \mathrm{LBW}\end{array}$ & $\begin{array}{l}\text { Development } \\
(\mathrm{N}=12) ; \\
\text { prospective } \\
\text { clinical } \\
\text { evaluation } \\
(\mathrm{N}=12) \\
\end{array}$ & $\begin{array}{l}\text { Model } \\
\text { outperformed } \\
\text { Calvert formula } \\
\text { with two feedback } \\
\text { concentrations. }\end{array}$ & $(71,72)$ \\
\hline & $\begin{array}{l}\text { Develop limited } \\
\text { sampling } \\
\text { strategy. }\end{array}$ & $\begin{array}{l}\text { Bayesian } \\
\text { pop-PK } \\
(\mathrm{CL})^{\mathrm{A}}\end{array}$ & $\begin{array}{l}\text { CL: BW, } \\
\text { Age, Sex, } \\
\text { S }_{\mathrm{CR}} ;\end{array}$ & $\begin{array}{l}\text { Development } \\
(\mathrm{N}=90) ; \\
\text { Validation } 1\end{array}$ & $\begin{array}{l}\text { All patients } \\
\text { achieved AUCs } \\
\text { close to target using }\end{array}$ & (89) \\
\hline
\end{tabular}




\begin{tabular}{|c|c|c|c|c|c|c|}
\hline & & & $\mathrm{V}_{1}, \mathrm{~V}_{2}: \mathrm{BSA}$ & $\begin{array}{l}\mathrm{N}=13) \\
\text { prospective } \\
\text { clinical } \\
\text { evaluation } \\
(\mathrm{N}=5)\end{array}$ & $\begin{array}{l}\text { the model-based } \\
\text { limited sampling } \\
\text { approach. }\end{array}$ & \\
\hline cyclophosphamide & $\begin{array}{l}\text { Reduce toxicity } \\
\text { in children } \\
\text { undergoing HCT. }\end{array}$ & $\begin{array}{l}\text { Bayesian } \\
\text { pop-PK } \\
\left(\mathrm{CL}_{\mathrm{NON}}\right. \\
\left.\mathrm{CL}_{\mathrm{IND}}\right)^{\mathrm{A}}\end{array}$ & $\mathrm{CL}_{\mathrm{NON}}$ : Age & $\begin{array}{l}\text { Development } \\
(\mathrm{N}=147) ; \\
\text { validation } 1 \\
(\mathrm{~N}=20) ; \\
\text { Clinical } \\
\text { evaluation } \\
(\mathrm{N}=20) ; \\
\text { prospective } \\
\text { clinical } \\
\text { evaluation } \\
(\mathrm{N}=50)\end{array}$ & $\begin{array}{l}\text { A software tool was } \\
\text { implemented in } \\
\text { clinical practice to } \\
\text { individualize dosing } \\
\text { in real-time. }\end{array}$ & $(80-83,90)$ \\
\hline \multirow[b]{2}{*}{ methotrexate } & $\begin{array}{l}\text { Early detection } \\
\text { of impaired } \\
\text { elimination. }\end{array}$ & $\begin{array}{l}\text { Bayesian } \\
\text { pop-PK: 2- } \\
\text { cmp }(B, \beta)^{\mathrm{A}}\end{array}$ & N/A & $\begin{array}{l}\text { Development } \\
(\mathrm{N}=7)\end{array}$ & $\begin{array}{l}\text { Bayesian limited } \\
\text { sampling strategy } \\
\text { (two samples) } \\
\text { allowed detection of } \\
\text { impaired clearance. }\end{array}$ & (92) \\
\hline & $\begin{array}{l}\text { Management of } \\
\text { high-dose } \\
\text { methotrexate } \\
\text { therapy with } \\
\text { leucovorine in } \\
\text { children/young } \\
\text { adults. }\end{array}$ & $\begin{array}{l}\text { Bayesian } \\
\text { pop-PK: 2- } \\
\text { cmp }(\mathrm{CL})^{\mathrm{A}}\end{array}$ & $\begin{array}{l}\text { Significant } \\
\text { difference in } \\
\text { CL between } \\
\text { normal and } \\
\text { reduced renal } \\
\text { function. }\end{array}$ & $\begin{array}{l}\text { Development } \\
(\mathrm{N}=240) \\
\text { retrospective } \\
\text { clinical } \\
\text { evaluation } \\
(\mathrm{N}=50)\end{array}$ & $\begin{array}{l}\text { A clinical software } \\
\text { tool was developed. } \\
\text { The tool showed } \\
\text { reasonable accuracy } \\
\text { for predicting } \\
\text { exposure. }\end{array}$ & $(84,85)$ \\
\hline pembrolizumab & $\begin{array}{l}\text { Evaluate impact } \\
\text { of PK modeling } \\
\text { on exposure, } \\
\text { target } \\
\text { engagement and } \\
\text { cost. }\end{array}$ & $\begin{array}{l}\text { Pop-PK: 2- } \\
\text { cmp }(C L)^{A} \\
(95)\end{array}$ & $\begin{array}{l}\text { CL: BW, } \\
\text { Albumin, } \\
\text { Baseline } \\
\text { tumor } \\
\text { burden, } \\
\text { eGFR, Sex, } \\
\text { Non-small } \\
\text { cell lung } \\
\text { cancer, Prior } \\
\text { pilimumab, } \\
\text { ECOG; } \\
\text { V: BW, } \\
\text { Albumin, } \\
\text { Sex, Prior } \\
\text { pilimumab } \\
\text { (95) }\end{array}$ & $\begin{array}{l}\text { Simulation } \\
\text { study }\end{array}$ & $\begin{array}{l}\text { Model-derived dose } \\
\text { banding can reduce } \\
\text { wastage and cost of } \\
\text { treatment while } \\
\text { maintaining optimal } \\
\text { exposure. }\end{array}$ & (66) \\
\hline
\end{tabular}




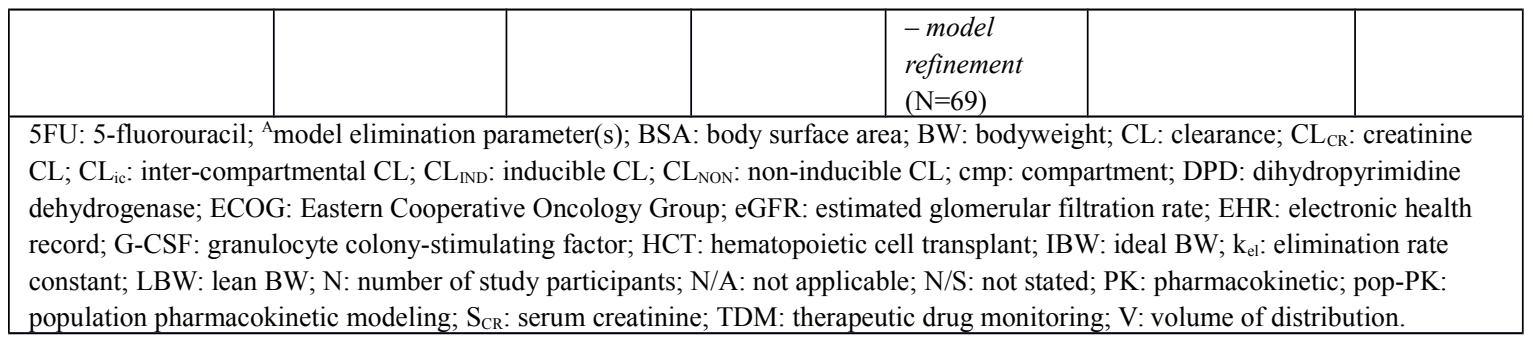

\section{FIGURE 1}

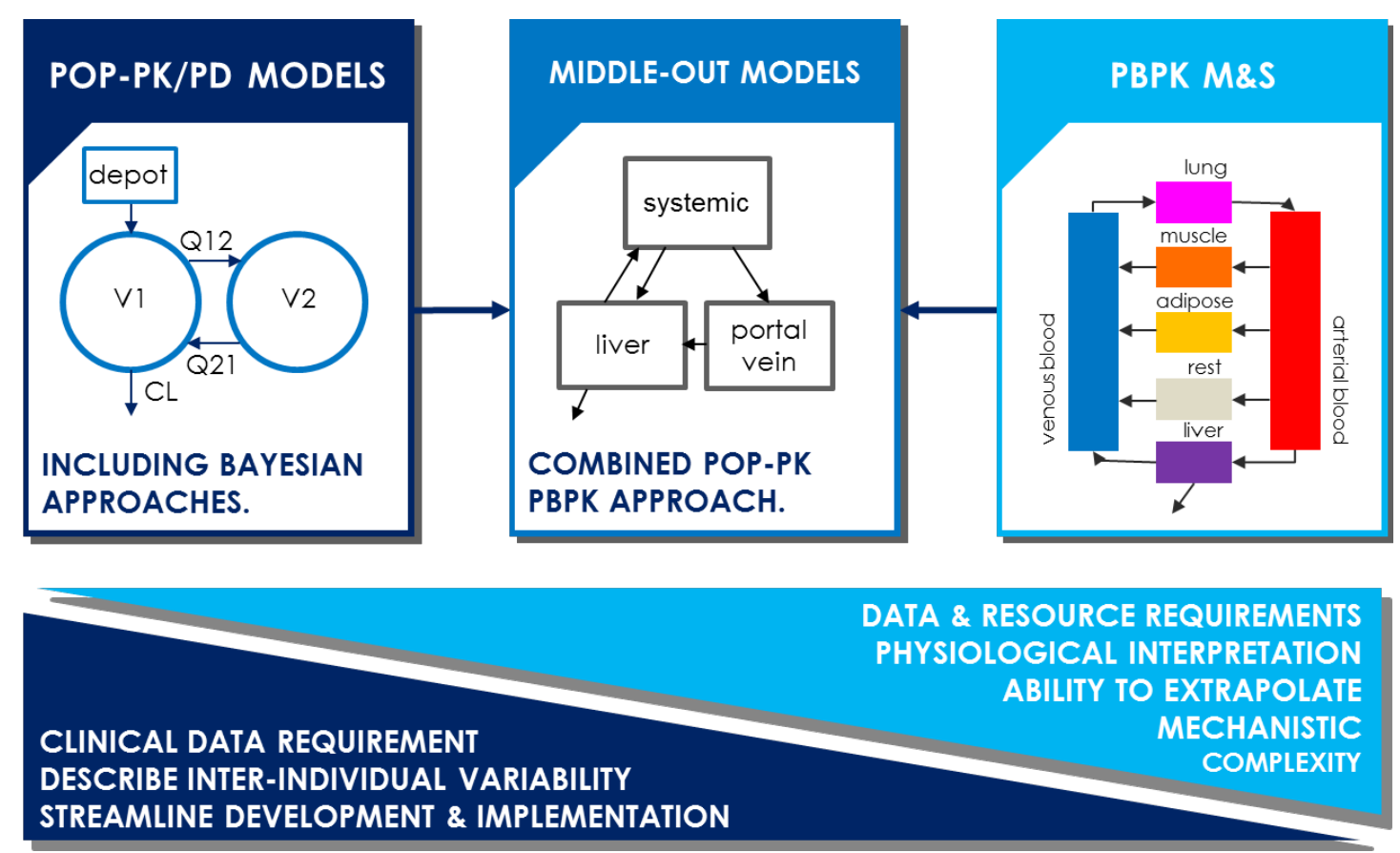

FIGURE 2 


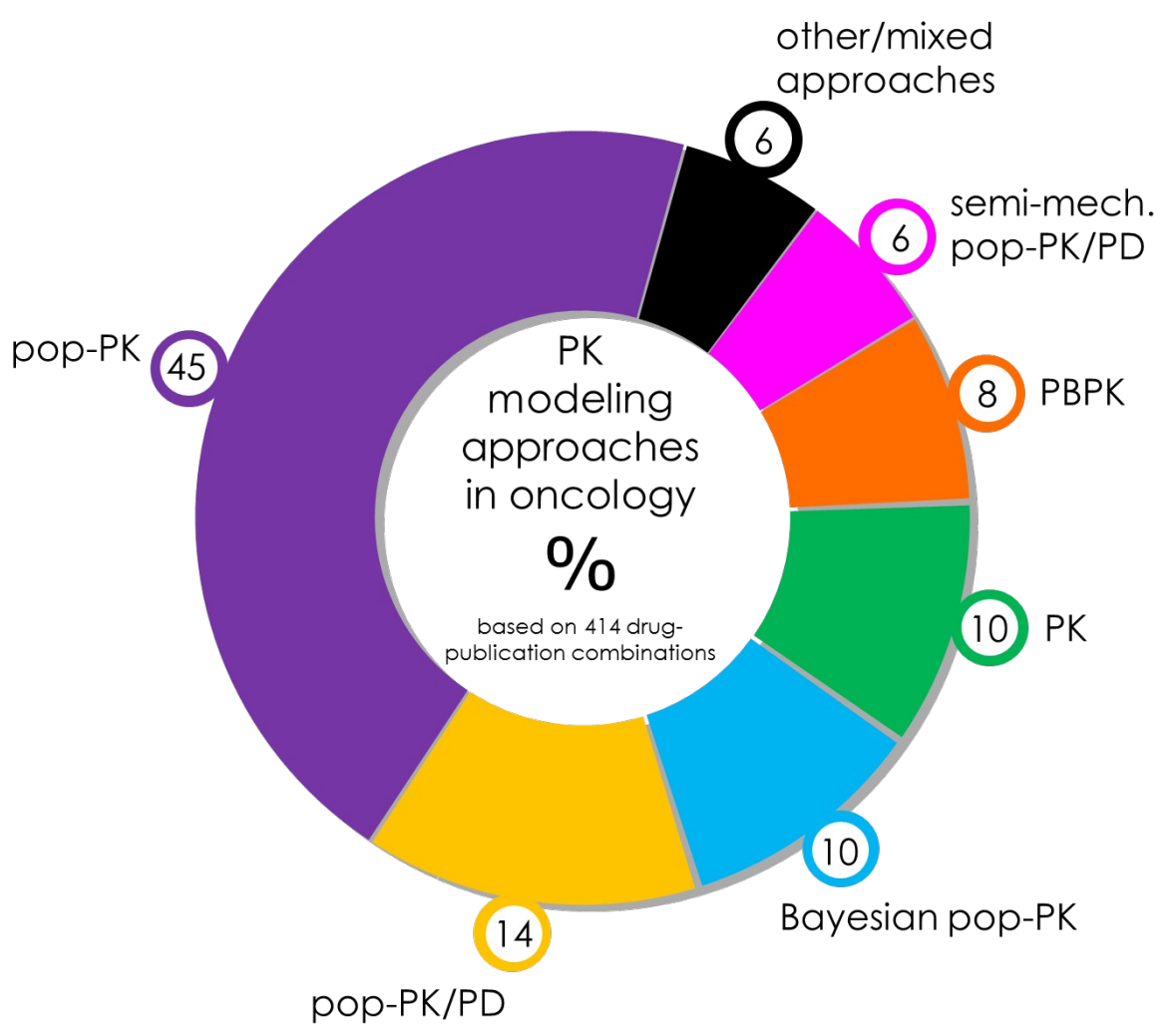

FIGURE 3

Applications of pharmacokinetic modeling in oncology

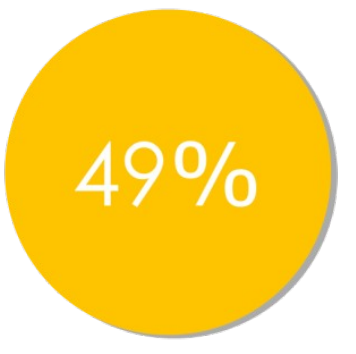

covariates

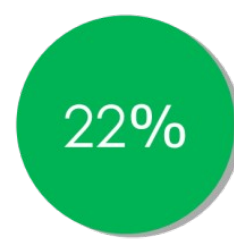

dose

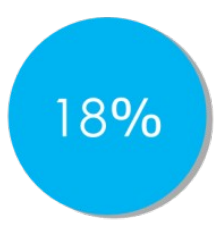

toxicity

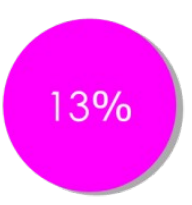

pediatrics

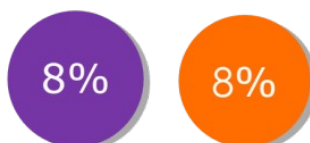

response metabolite kinetics

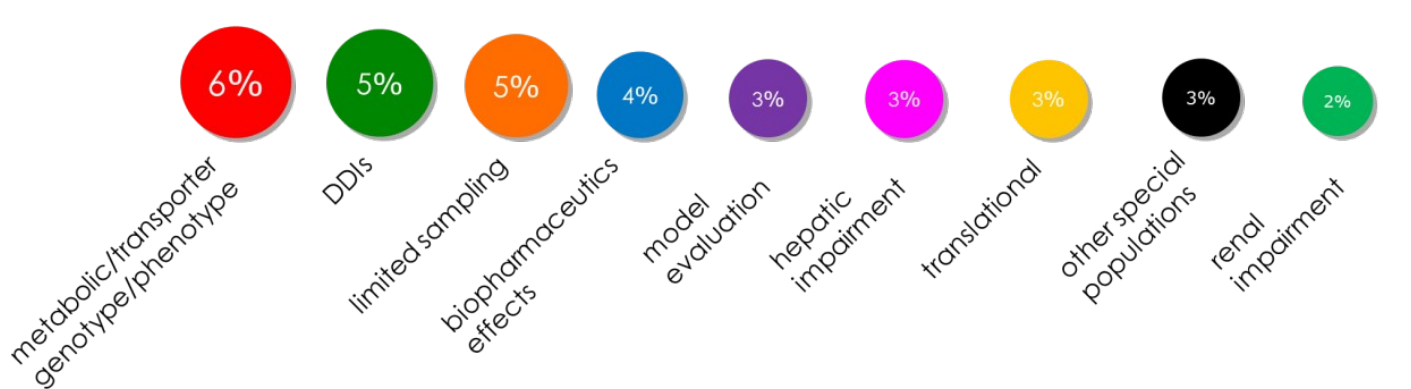

sample of 414 drug-publication combinations 
Pharmacokinetic models for precision dosing in oncology

FIGURE 4 


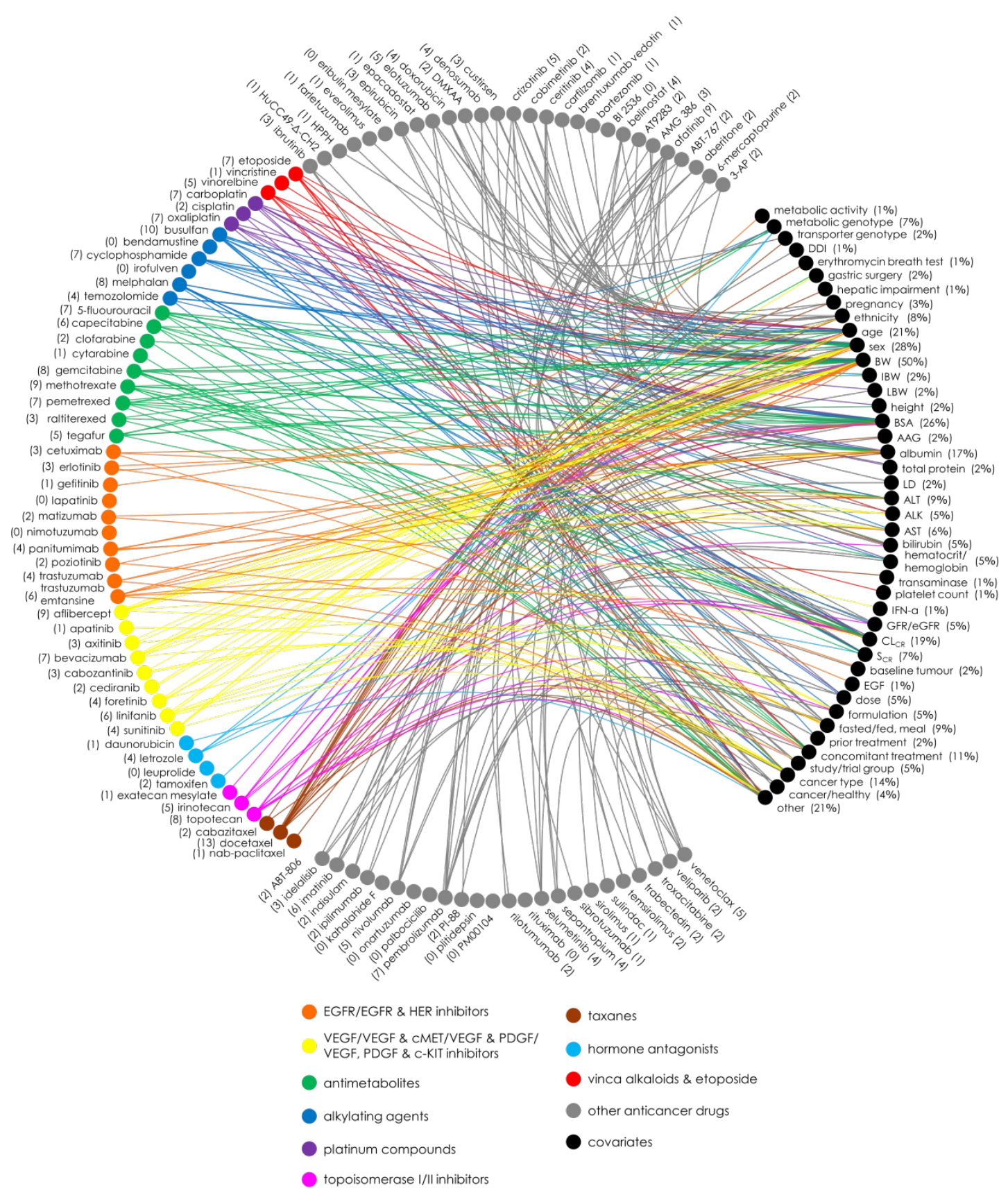

FIGURE 5 

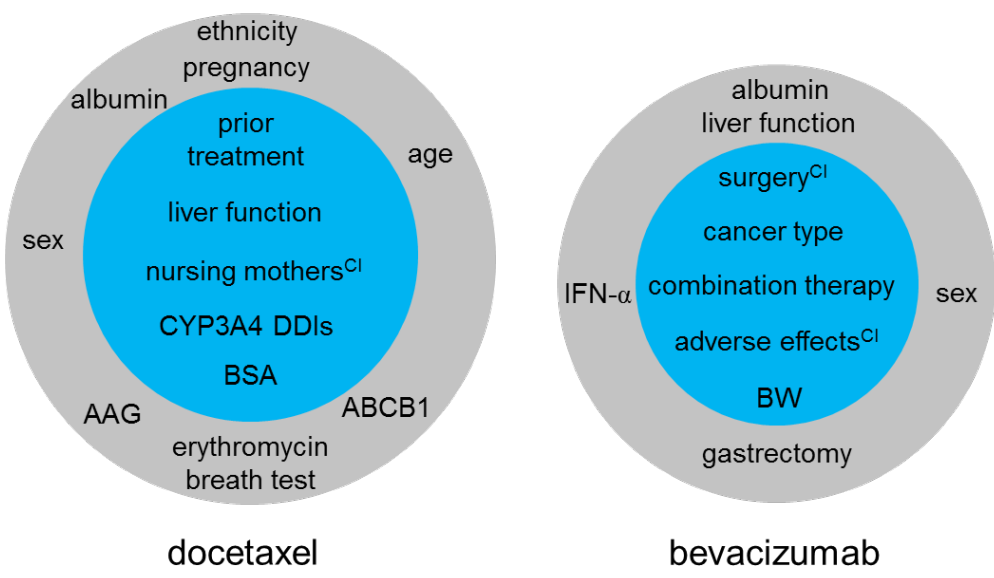

bevacizumab

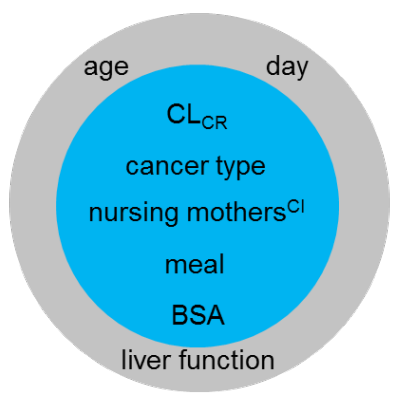

capecitabine

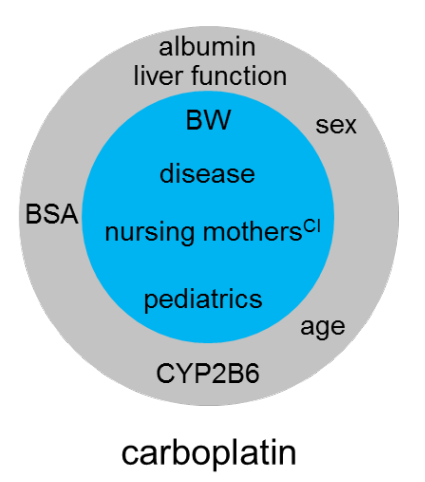

FDA drug label peer-reviewed
literature
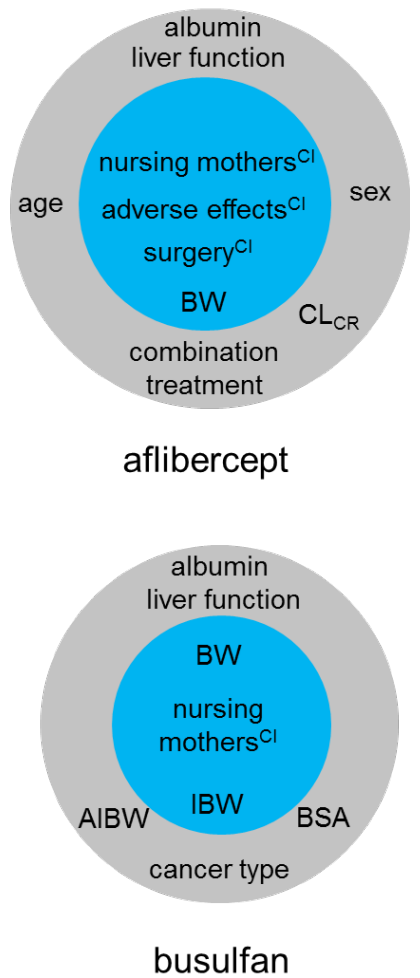


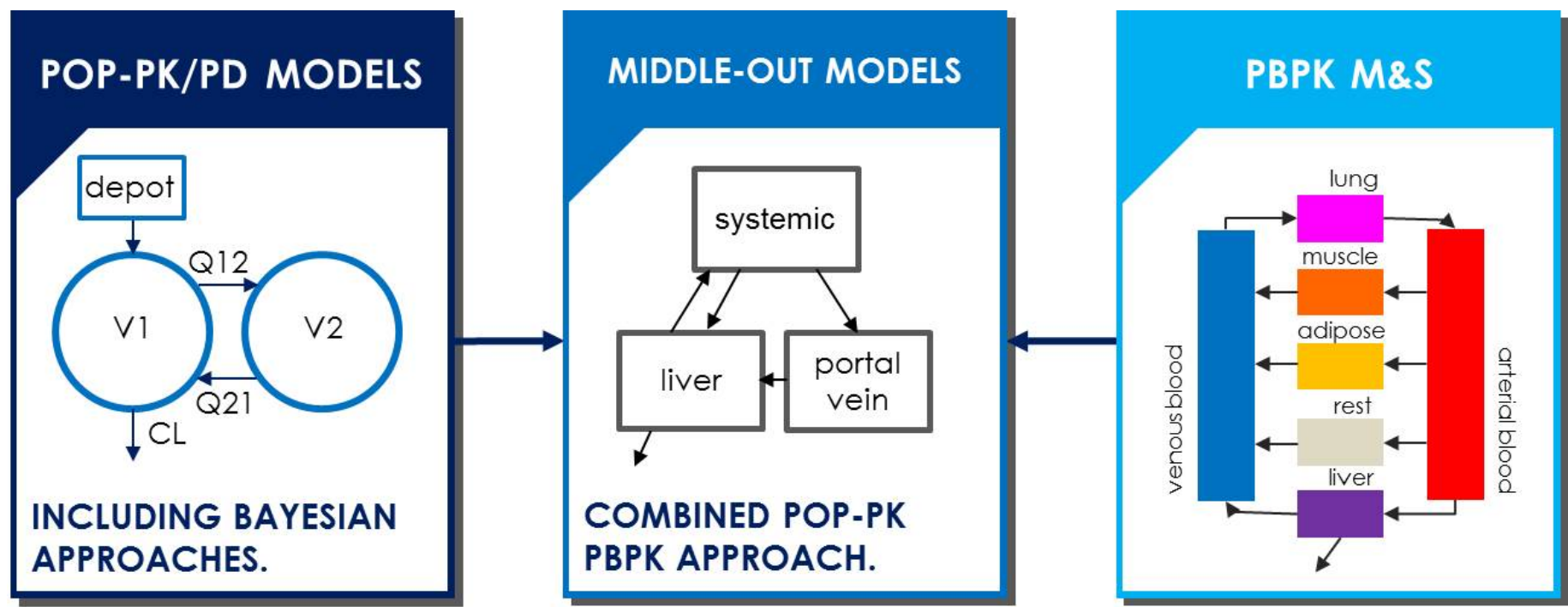

DATA \& RESOURCE REQUIREMENTS PHYSIOLOGICAL INTERPRETATION ABILITY TO EXTRAPOLATE CLINICAL DATA REQUIREMENT MECHANISTIC DESCRIBE INTER-INDIVIDUAL VARIABILITY COMPLEXITY STREAMLINE DEVELOPMENT \& IMPLEMENTATION 


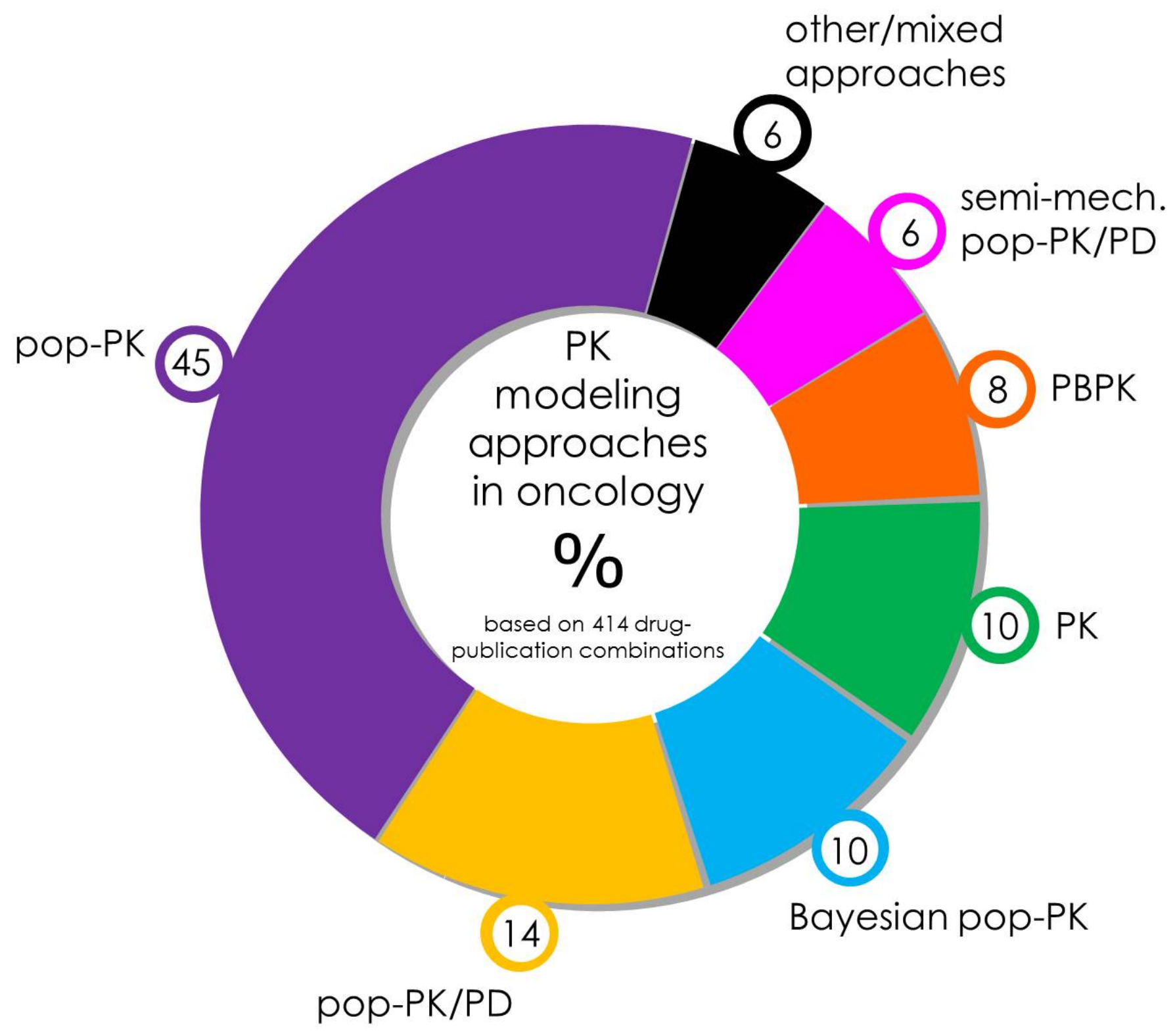


Applications of pharmacokinetic modeling in oncology

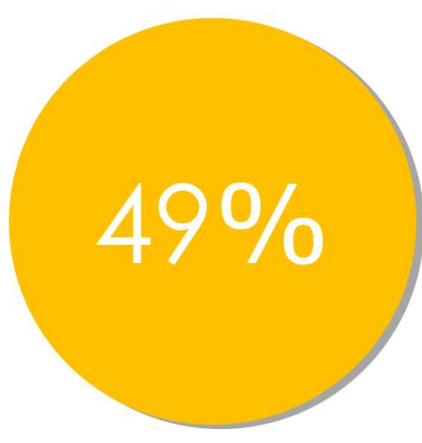

covariates

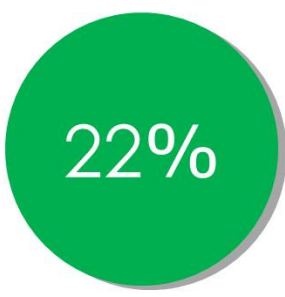

dose

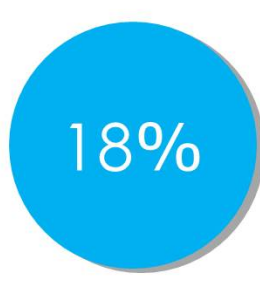

toxicity

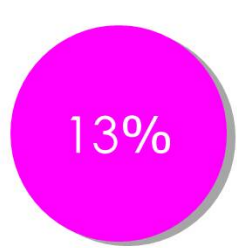

pediatrics

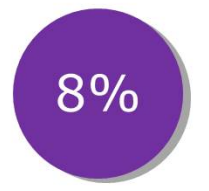

response

\section{$8 \%$}

metabolite kinetics

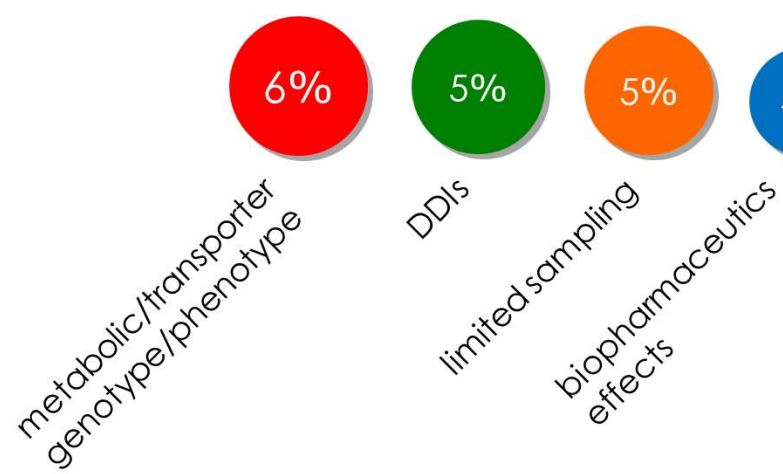

\section{$4 \%$}




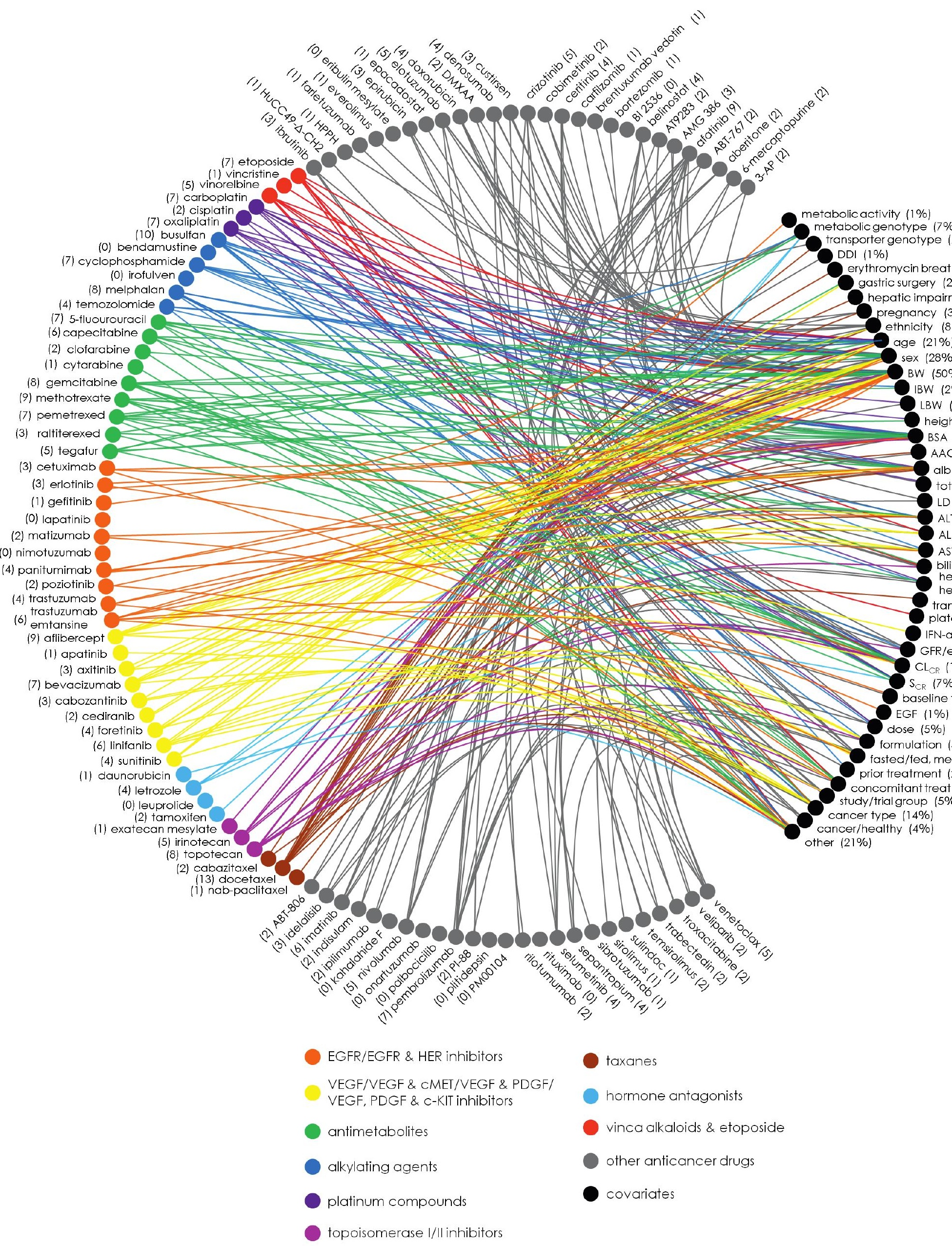


Pharmacokinetic models for precision dosing in oncology - Supplementary appendix

\section{Supplementary Appendix: Role of pharmacokinetic modeling and simulation in precision dosing of anticancer} drugs

Adam S. Darwich ${ }^{1}$, Kayode Ogungbenro ${ }^{1}$, Oliver J.D. Hatley², Amin Rostami-Hodjegan ${ }^{1,2}$

${ }^{I}$ Centre for Applied Pharmacokinetic Research, Division of Pharmacy and Optometry, University of Manchester, Manchester, UK.

${ }^{2}$ Certara, Blades Enterprise Building, Sheffield, UK.

\section{Table of contents}

Methods

page 2

Results

page 5

Table A1. Pharmacokinetic modelling of oncology drugs/investigated drugs.

page 5

References

page 28 
Pharmacokinetic models for precision dosing in oncology - Supplementary appendix

\section{Methods}

A literature search was carried out using PubMed (https://www.ncbi.nlm.nih.gov/pubmed; 1950 - March 2017) to identify a sample set of publications including PK modeling of anticancer drugs. The inclusion criteria consisted of PK modeling of oncology drugs aimed at either predicting human exposure or based on clinical data. Studies based on pre-clinical data only or pharmacodynamic modeling only were excluded during screening of publications. To capture a broad variety of modeling applications in oncology the search terms included: "physiologically based pharmacokinetic", "pharmacokinetic", "PBPK", "PKPD", and "pharmacodynamic", and "model", "modeling", and "modelling", and "oncology", "cancer", "anti-cancer", "tumor", "tumor", "anti-tumor" or "anti-tumor", with filtering based on human species. The search yielded 1,749 publications. Additional publications were identified through references in identified publications, review articles and key literature. The final dataset included 393 publications.

The identified set of publications was categorized based on main and secondary study aims, including:

- toxicity: adverse effects/toxicity;

- bioequivalence: PK modeling of bioequivalence studies;

- biopharmaceutics effects: biopharmaceutics effects, other than food and formulation effects;

- covariates: PK covariates, or otherwise included covariates in the model;

- DDIs: metabolic/transporter drug-drug interactions;

- dose: investigating multiple doses, optimal dose, or dose individualization; 
Pharmacokinetic models for precision dosing in oncology - Supplementary appendix

- efficacy: drug efficacy/response;

- ethnicity: differences in PK based on ethnicity;

- evaluation/validation: assessment or validation of PK model performance;

- food effects: impact of prandial state and/or meal properties on PK;

- formulation: impact of formulation on PK;

- genotype: metabolic/transporter genotype (and $\alpha_{1}$-acid glycoprotein genotype) effects on PK;

- Surgery: impact of surgery on PK (including gastric and cytoreductive surgery);

- hepatic impairment: PK in hepatically impaired subjects, assessing impact of hepatic impairment on PK;

- investigational: main focus to investigate PK of the selected drug;

- limited: optimal limited sampling strategies;

- metabolite: metabolite PK;

- other special populations: PK in other special populations (including: elderly, renal replacement therapy and postmenopausal women);

- pediatrics: pediatric drug exposure;

- phenotype: metabolic/transporter phenotype effects on PK;

- renal impairment: PK in renally impaired subjects, assessing impact of renal impairment on PK;

- $\quad$ sex: impact of sex on PK.

Where applicable, identified PK covariates were recorded, either including the full covariate model or a summary of covariates and the PK parameter the effect was modeled on. Additional recording of information included: investigated drug, modeling approach used for development and/or application, and study population(s). Data was analyzed through graphical presentation. 
Pharmacokinetic models for precision dosing in oncology - Supplementary appendix

\section{Results}

Table A1 presents the summary information of the literature search on pharmacokinetic (PK) modeling of anticancer drugs. The search results included 393 peer-reviewed publications of 177 approved or investigated oncology drugs, or 414 unique study-drug combinations.

\begin{tabular}{|c|c|c|c|c|c|}
\hline Drug & Study population & Modelling approach & Identified pharmacokinetic covariates & $\begin{array}{l}\text { Main/secondary aims of } \\
\text { study }\end{array}$ & $\begin{array}{l}\text { References } \\
\end{array}$ \\
\hline $\begin{array}{l}\text { 2-[1-hexyloxyethyl]-2-devinyl } \\
\text { pyropheophorbide-a (HPPH) }\end{array}$ & $\begin{array}{l}\text { Esophageal cancer, basal } \\
\text { cell cancer, lung cancer }\end{array}$ & Pop-PK/PD & CL - Age & Toxicity, Covariates & $\begin{array}{l}\text { (Bellnier et al. } \\
\underline{2003})\end{array}$ \\
\hline $260 \mathrm{F9},{ }^{111} \mathrm{In}$ & Breast cancer & PK & & Investigational & $\begin{array}{l}\text { (Griffin et al. } \\
\text { 1989) }\end{array}$ \\
\hline $\begin{array}{l}\text { 3-aminopyridine-2- } \\
\text { carboxaldehyde } \\
\text { thiosemicarbazone (3-AP) }\end{array}$ & $\begin{array}{l}\text { Advanced metastatic } \\
\text { tumors }\end{array}$ & Pop-PK & $\begin{array}{l}\mathrm{CL}[\mathrm{L} / \mathrm{h}]=25.0, \mathrm{CL} \text { in PatientsReceivingOneCycleOnly: } 17.0, \\
\mathrm{~V}_{2}[\mathrm{~L}]-\text { Female }=19.0, \text { Male }=34.8\end{array}$ & Covariates & $\begin{array}{l}\text { (Kolesar et al. } \\
\text { 2011) }\end{array}$ \\
\hline $\begin{array}{l}\text { 4'demethylepidodophyllotoxin } \\
\text { 9-(4,6-O-ethylidene-Beta-D- } \\
\text { glucopyranoside) }\end{array}$ & Advanced cancer & PK & & Investigational & $\begin{array}{l}\text { (Pelsor et al.. } \\
\text { 1978) }\end{array}$ \\
\hline $\begin{array}{l}\text { 5,6-dimethylxanthenone-4- } \\
\text { acetic acid (DMXAA) }\end{array}$ & Solid tumors & Pop-PK/PD & $\begin{array}{l}\mathrm{V}_{\mathrm{m}}[\mu \mathrm{mol} / \mathrm{L} / \mathrm{h}]=112 \cdot(1+0.474 \cdot(2-\text { Sex }[\text { Female: } 1, \text { Male: } 2)), \\
\mathrm{V}_{1}[\mathrm{~L}]=8.19 \cdot(B S A / 1.8)^{0.857}\end{array}$ & Efficacy, Covariates & $\begin{array}{l}\text { (Li et al.. } \\
\underline{2008)}\end{array}$ \\
\hline \multirow[t]{3}{*}{ 5-fluorouracil } & Cancer $(\mathrm{N} / \mathrm{S})$ & Semi-mech. PK & & Investigational & $\begin{array}{l}\text { (Robinson et } \\
\text { al.., 1988) }\end{array}$ \\
\hline & Colorectal cancer & PK & & Investigational & (Wattanatorn \\
\hline & Metastatic colorectal & Bayesian pop-PK & $\mathrm{CL}[\mathrm{L} / \mathrm{h}]=60.2 \cdot$ Female +65.0 & Investigational, Covariates & $\begin{array}{l}\text { (Bressolle et } \\
\text { al., 1999) }\end{array}$ \\
\hline
\end{tabular}


Pharmacokinetic models for precision dosing in oncology - Supplementary appendix

\begin{tabular}{|c|c|c|c|c|c|}
\hline & cancer & & Circadian rhythm (cosine model) & & \\
\hline & Cancer (N/S) & $\mathrm{PK} / \mathrm{PD}$ & & Efficacy, Dose & $\begin{array}{l}\text { (Gardner, } \\
\underline{2002)}\end{array}$ \\
\hline & $\begin{array}{l}\text { Dukes' colorectal } \\
\text { adenocarcinoma }\end{array}$ & PK & & Limited sampling & $\begin{array}{l}\text { (Di Paolo et } \\
\text { al., 2002) }\end{array}$ \\
\hline & Colorectal cancer & Bayesian pop-PK & $\begin{array}{l}\mathrm{V}[\mathrm{L}]=0.266 \cdot B W \\
\mathrm{CL}[\mathrm{L} / \mathrm{h}]=1.210 \cdot I B W\end{array}$ & Covariates & $\begin{array}{l}\text { (Climente- } \\
\text { Marti et al.. } \\
2003)\end{array}$ \\
\hline & Cancer (N/S) & Pop-PK & & Metabolite & $\begin{array}{l}\text { (Coustere et } \\
\text { al., 1991) }\end{array}$ \\
\hline & Breast cancer & $\begin{array}{l}\text { Semi-mech. Pop- } \\
\text { PK/PD }\end{array}$ & $\begin{array}{l}\mathrm{V}_{\max }[\mathrm{mg} / \mathrm{L} / \mathrm{h}]=94.8, C L_{C R}: 0.24 \\
\mathrm{~K}_{\mathrm{m}}[\mathrm{mg} / \mathrm{L}]=21.2, \text { Albumin: } 1.87 \\
\mathrm{~V}[\mathrm{~L}]=23.2, \text { BSA } 1.74: 0.377, B S A>1.74: 1.32\end{array}$ & Toxicity, Covariates & $\begin{array}{l}\text { (Sandstrom et } \\
\text { al. 2006) }\end{array}$ \\
\hline & Breast cancer & Pop-PK & & Investigational & $\begin{array}{l}\text { (Sandstrom et } \\
\text { al. 1996) }\end{array}$ \\
\hline & Colorectal cancer & Pop-PK & Caution when dosing BSA in women & Metabolite, Covariates & (Woloch et al.. \\
\hline & Mixed cancer & Bayesian pop-PK & & Metabolic genotype (DPD) & $\begin{array}{l}\text { (van } \\
\text { Kuilenburg et } \\
\text { al..2012) } \\
\end{array}$ \\
\hline 6-mercaptopurine & $\begin{array}{l}\text { Acute lymphoblastic } \\
\text { leukemia }\end{array}$ & Pop-PK & $\begin{array}{l}\mathrm{F}_{\mathrm{M} 3}=0.019 \cdot 2.56^{\text {TPMTMutation }} \\
\mathrm{CL}_{6-\mathrm{TGNS}}\left[\mathrm{h}^{-1}\right]=0.00914 \cdot B S A^{1.16}\end{array}$ & $\begin{array}{l}\text { Pediatrics, Metabolic genotype } \\
\text { (TPMT), Metabolite, } \\
\text { Covariates }\end{array}$ & $\begin{array}{l}\text { (Hawwa et al. } \\
\text { 2008) }\end{array}$ \\
\hline 9-aminocamptothecin & Solid tumors & PK & & Investigational & $\begin{array}{l}\text { (Rubin et al., } \\
\text { 1995) }\end{array}$ \\
\hline Abexinostat & Solid tumors, lymphoma & Pop-PK/PD & & Dose, Toxicity & $\begin{array}{l}\text { (Chalret du } \\
\text { Rieu et al.. } \\
\text { 2014a) }\end{array}$ \\
\hline Aberitorone & Prostate cancer & Pop-PK & $\begin{array}{l}\mathrm{CL} / \mathrm{F}[\mathrm{L} / \mathrm{h}]=2,240 \cdot(1+\text { Healthy } \cdot(-0.308)), \\
\text { Fed state/meal properties }\end{array}$ & Food effects, Covariates & $\begin{array}{l}\text { (Stuyckens et } \\
\text { al.., 2014) }\end{array}$ \\
\hline ABT-767 & $\begin{array}{l}\text { High grade severe ovarian } \\
\text { cancer, primary peritoneal } \\
\text { cancer, fallopian tube } \\
\text { cancer }\end{array}$ & Pop-PK & $\begin{array}{l}\mathrm{CL} / \mathrm{F}[\mathrm{L} / \mathrm{h}]=7.34 \cdot\left(\text { Albumin }_{i} / \text { Albumin }_{\text {Median }}\right)^{0.651} \\
\mathrm{k}_{\mathrm{a}}\left[\mathrm{h}^{-1}\right]=1.45 \cdot 0.54^{\text {Fed }}\end{array}$ & Covariates & $\begin{array}{l}\text { (Mittapalli et } \\
\text { al.. 2017) }\end{array}$ \\
\hline ABT-806 & $\begin{array}{l}\text { Head and neck squamous } \\
\text { cancer, non-small cell lung } \\
\text { cancer, colorectal cancer }\end{array}$ & Pop-PK & $\begin{array}{l}\mathrm{CL}[\mathrm{L} / \mathrm{day}]=0.011 \cdot\left(\text { Albuminin }_{i} / \text { Albumin }_{\text {Median }}\right)^{-0.924} \\
\mathrm{~V}_{1}[\mathrm{~L}]=3.47 \cdot\left(\mathrm{BW}_{\mathrm{i}} / \mathrm{BW}_{\text {Median }}\right)^{0.542}\end{array}$ & Dose, Covariates & $\begin{array}{l}\text { (Sharma et al.. } \\
\underline{2015)}\end{array}$ \\
\hline & Cancer (N/S) & PBPK & & Pediatrics & $\begin{array}{l}\text { (Walsh et al.. } \\
\underline{2016)}\end{array}$ \\
\hline Actinomycin D & Cancer (N/S) & Pop-PK & & Pediatrics & $\begin{array}{l}\text { (Edwards et } \\
\text { al., 2012) }\end{array}$ \\
\hline Afatinib & Solid tumors & Pop-PK & $\begin{array}{l}\mathrm{CL} / \mathrm{F}=42.3 \cdot(\mathrm{BW} / 62)^{0.595} \cdot\left(1+0.00484 \cdot\left(\mathrm{CL}_{\mathrm{CR}}-120\right) \cdot 0.871^{\text {Female }} \cdot(1-\right. \\
0.00436 \cdot(\text { TotalProtein- } 72)) \\
\mathrm{F}_{1}=1 \cdot(\text { Dose } / 70)^{0.485 \cdot \text { Dose } \_70 \mathrm{mg} \cdot} \cdot 0.739^{\text {Food }} \cdot \theta_{\text {ECOG }} \cdot(1+0.000331 \cdot \\
(\text { LactateDehydrogenase- } 241)) \cdot\left(1+\theta_{\text {AlkalinePhosphate }} \cdot(\text { AlkalinePhosphate-251) })\right. \\
\cdot \text { CancerType, } \\
\mathrm{V}_{2} / \mathrm{F}[\mathrm{L}]=456 \cdot(\mathrm{BW} / 62)^{0.899}\end{array}$ & Food effects, Covariates & $\begin{array}{l}\text { (Freiwald et } \\
\text { al.. 2014) }\end{array}$ \\
\hline
\end{tabular}


Pharmacokinetic models for precision dosing in oncology - Supplementary appendix

\begin{tabular}{|c|c|c|c|c|c|}
\hline & & & ECOG: Eastern Cooperative Oncology Group & & \\
\hline \multirow[t]{2}{*}{ Aflibercept } & Advanced solid tumors & Pop-PK & 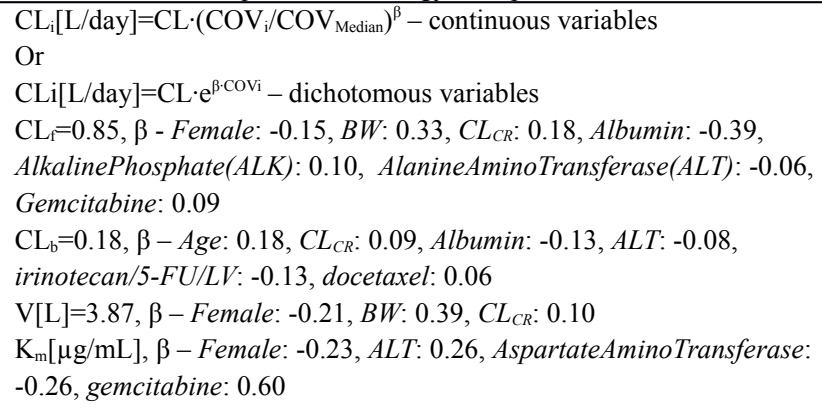 & Covariates, Dose & $\begin{array}{l}\text { (Thai et al.. } \\
\text { 2013) }\end{array}$ \\
\hline & Healthy volunteers & Semi-mech. Pop-PK & & Investigational & $\begin{array}{l}\text { (Thai et al.. } \\
\text { 2011) }\end{array}$ \\
\hline Alectinib & $\begin{array}{l}\text { Healthy volunteers, cancer } \\
(\mathrm{N} / \mathrm{S})\end{array}$ & PBPK & & $\begin{array}{l}\text { Food effects, Biopharmaceutics } \\
\text { effects }\end{array}$ & $\underline{\underline{2016})}$ \\
\hline All-trans-retinoic acid & Cancer (N/S) & PBPK & & DDIs & $\begin{array}{l}\text { (Jing et al.. } \\
2017)\end{array}$ \\
\hline Alvespimycin & $\mathrm{N} / \mathrm{A}$ & PBPK & & Inter-species extrapolation & $\begin{array}{l}\text { (Hu et al... } \\
2014) \\
\end{array}$ \\
\hline Amatuximab & $\begin{array}{l}\text { Malignant pleural } \\
\text { mesothelioma }\end{array}$ & Pop-PK & & Dose & (Gupta et al.. \\
\hline AMD3100 & Healthy volunteers & Pop-PK/PD & & Efficacy & $\begin{array}{l}\text { (Lack et al.. } \\
\text { 2005) }\end{array}$ \\
\hline AMG 386 & $\begin{array}{l}\text { Advanced solid tumors, } \\
\text { advanced ovarian cancer }\end{array}$ & Pop-PK/PD & $\begin{array}{l}\mathrm{CL}[\mathrm{L} / \mathrm{h}]=0.0722+0.502 \cdot \mathrm{CL}_{\mathrm{CR}}, \\
\mathrm{V}_{\mathrm{c}}[\mathrm{L}]=3.74 \cdot\left(B W_{i} / B W_{\text {reference }}\right) 0.502, \text { Sex: } 0.83\end{array}$ & Efficacy, Covariates & $\underline{\underline{2012})}$ \\
\hline Amrubicin & $\begin{array}{l}\text { Small cell and non-small } \\
\text { cell lung cancer }\end{array}$ & Pop-PK & & Limited sampling & $\begin{array}{l}\text { (Makino et al. } \\
\underline{2016)}\end{array}$ \\
\hline Anastrozole & Healthy volunteers & Pop-PK & & Bioequivalence & $\begin{array}{l}\text { (Noh et al.., } \\
\text { 2012) }\end{array}$ \\
\hline \multirow{3}{*}{ Anti-CD66 antibody } & Acute leukemia & PBPK & & Investigational & $\begin{array}{l}\text { (Kletting et al. } \\
\text { 2010) }\end{array}$ \\
\hline & Acute leukemia & PBPK & & Investigational & $\begin{array}{l}\text { (Kletting et al. } \\
\text { 2015) }\end{array}$ \\
\hline & Cancer (N/S) & PBPK & & Investigational & $\begin{array}{l}\text { (Maass et al.. } \\
\text { 2015) }\end{array}$ \\
\hline Apatinib & $\begin{array}{l}\text { Healthy volunteers, solid } \\
\text { tumors }\end{array}$ & Pop-PK & $\begin{array}{l}\mathrm{CL} / \mathrm{F}[\mathrm{L} / \mathrm{h}]=57.8, \text { DiseaseState }:-0.390, \\
\mathrm{k}_{\mathrm{a}}\left[\mathrm{h}^{-1}\right]=0.0848, \text { DiseaseState }:-0.517\end{array}$ & Covariates & $\begin{array}{l}\text { (Yuet al.. } \\
\underline{2017 \mathrm{~b})}\end{array}$ \\
\hline AT9283 & $\begin{array}{l}\text { Solid tumors, relapsed or } \\
\text { refractory leukemia }\end{array}$ & Pop-PK & $\begin{array}{l}\mathrm{CL}[\mathrm{L} / \mathrm{h}]=32.2 \cdot\left(B W_{i} / B W_{\text {reference }}\right)^{0.75} \cdot\left(G F R_{i} / G F R_{\text {reference }}\right)^{0.452} \\
\mathrm{~V}_{\mathrm{c}}[\mathrm{L}]=58.5 \cdot\left(B W_{i} / B W_{\text {reference }}\right)^{1}\end{array}$ & Pediatrics, Covariates, Dose & $\begin{array}{l}\text { (Duong et al.. } \\
\underline{2017)}\end{array}$ \\
\hline Axitinib & Non-small cell lung cancer & Pop-PK & $\begin{array}{l}\mathrm{CL}[\mathrm{L} / \mathrm{h}]=20.1, \text { Female: }-0.349 \\
\mathrm{~V}_{\mathrm{c}}[\mathrm{L}]=56.2, \text { BW: } 0.933 \\
\mathrm{~F}=0.663, \text { Food: }-0.263 \\
\mathrm{Ka}\left[\mathrm{h}^{-1}\right]=1.26, \text { Food: }-0.653\end{array}$ & Food effects, Covariates & $\begin{array}{l}\text { (Tortorici et } \\
\text { al.. 2014) }\end{array}$ \\
\hline
\end{tabular}


Pharmacokinetic models for precision dosing in oncology - Supplementary appendix

\begin{tabular}{|c|c|c|c|c|c|}
\hline B72.3 & Colon cancer & $\mathrm{PK}$ & & Investigational & $\begin{array}{l}\text { (Reilly et al.., } \\
1993 \text { ) }\end{array}$ \\
\hline Barasertib & Solid tumors & Pop-PK/PD & & Toxicity, Dose & 2012) \\
\hline Belinostat & $\begin{array}{l}\text { Peripheral T-cell } \\
\text { lymphoma }\end{array}$ & Pop-PK/PD & $\begin{array}{l}\theta_{\mathrm{i}}=\theta \cdot\left(\mathrm{COV}_{\mathrm{i}} / \mathrm{COV}_{\text {median }}\right)^{\beta C O V} \\
\mathrm{CL}[\mathrm{L} / \mathrm{h}]=106.03, L_{C R}: 0.3799, \text { UGTgenotype: } 0.8475, \text { Albumin: }-0.3897, \\
\mathrm{~V}[\mathrm{~L}]=31.82, B W: 0.3848\end{array}$ & $\begin{array}{l}\text { Metabolic genotype } \\
\text { (UGT1A1), Toxicity, } \\
\text { Covariates }\end{array}$ & $\begin{array}{l}\text { (Peer et al.. } \\
\underline{2016)}\end{array}$ \\
\hline Bendamustine & $\begin{array}{l}\text { Relapsed/refractory acute } \\
\text { leukemia }\end{array}$ & Pop-PK/PD & & Pediatrics, Toxicity, Covariates & $\begin{array}{l}\text { (Darwish et } \\
\text { al., 2014) } \\
\end{array}$ \\
\hline \multirow{6}{*}{ Bevacizumab } & Colorectal cancer & Pop-PK & & Covariates & $\begin{array}{l}\text { (Panoilia et al.. } \\
\underline{2015)}\end{array}$ \\
\hline & $\begin{array}{l}\text { Metastatic colorectal } \\
\text { cancer }\end{array}$ & Pop-PK & & Investigational & $\begin{array}{l}\text { (Caulet et al.. } \\
\text { 2016) }\end{array}$ \\
\hline & $\begin{array}{l}\text { Solid tumors, non-small } \\
\text { cell lung cancer, hormone } \\
\text { refractory prostate cancer, } \\
\text { breast cancer, renal cell } \\
\text { cancer, pancreatic cancer, } \\
\text { metastatic colorectal } \\
\text { cancer }\end{array}$ & Pop-PK & $\begin{array}{l}\mathrm{CL}[\mathrm{mL} / \mathrm{h}]=8.6 \cdot\left(\text { BW }_{i} / 70\right)^{0.589} \cdot\left(\text { Albumin }_{i i} / 39\right)^{-} \\
0.473 \cdot\left(\ln \left(\text { AlkalinePhosphatase }_{i} / \ln (109)\right)^{0.312} \cdot(1.14 \text { for Males }) \cdot(0.844 \text { for }\right. \\
\text { InterferonAlpha }) \\
\mathrm{V} 1[\mathrm{~mL}]=2678 \cdot\left(\text { BW }_{i} / 70\right)^{0.470} \cdot(1.18 \text { for Males })\end{array}$ & Covariates & $\begin{array}{l}\text { (Han et al... } \\
\text { 2016a) }\end{array}$ \\
\hline & $\begin{array}{l}\text { Refractory sarcomas, } \\
\text { primary CNS tumors, } \\
\text { osteosarcoma, metastatic } \\
\text { soft-tissue sarcoma } \\
\end{array}$ & Pop-PK & $\begin{array}{l}\mathrm{CL}[\mathrm{mL} / \mathrm{h}]=9.9 \cdot\left(B_{i} / 70\right)^{0.75} \cdot\left(\text { Albumin }_{i i} / 39\right)^{-0.3} \cdot(1.11 \text { for Males }) \cdot(0.725 \text { for } \\
\text { CNStumours }) \\
\mathrm{V} 1[\mathrm{~mL}]=2850 \cdot\left(\text { BW }_{i} / 70\right)^{0.701} \cdot(1.14 \text { for Males }) \cdot(0.854 \text { for } \text { CNStumours })\end{array}$ & Pediatrics, Dose, Covariates & $\begin{array}{l}(\text { Han et al... } \\
\text { 2016b) }\end{array}$ \\
\hline & Gastric cancer & Bayesian pop-PK & CL: BW, Gastrectomy, Albumin & Covariates & $\begin{array}{l}\text { (Han et al.. } \\
\underline{\underline{2014}}\end{array}$ \\
\hline & Cancer (N/S) & Semi-mech. PK & & Investigational & $\begin{array}{l}\text { (Stefanini et } \\
\text { al., 2010) } \\
\end{array}$ \\
\hline \multirow[t]{2}{*}{ BI 2536} & $\begin{array}{l}\text { Advanced non-resectable } \\
\text { and/or metastatic refractory } \\
\text { solid tumors }\end{array}$ & $\begin{array}{l}\text { Semi-mech. Pop- } \\
\text { PK/PD }\end{array}$ & & Toxicity, Dose, Covariates & $\begin{array}{l}\text { (Soto et al.. } \\
\underline{2010)}\end{array}$ \\
\hline & Non-small cell lung cancer & $\begin{array}{l}\text { Semi-mech. Pop- } \\
\text { PK/PD }\end{array}$ & & Evaluation/validation, Toxicity & $\begin{array}{l}\text { (Soto et al.. } \\
\underline{2011 b)}\end{array}$ \\
\hline BI 893923 & $\mathrm{~N} / \mathrm{A}$ & Pop-PK/PD & & $\begin{array}{l}\text { Inter-species extrapolation, } \\
\text { Dose }\end{array}$ & $\begin{array}{l}\text { (Titze et al.. } \\
\text { 2017) }\end{array}$ \\
\hline Bortezomib & Leukemia & Pop-PK & $\mathrm{CL}, \mathrm{Q}_{3}: B S A$ & Pediatrics, Covariates & $\begin{array}{l}\text { (Hanley et al.. } \\
\text { 2016) }\end{array}$ \\
\hline Brentuximab vedotin & $\begin{array}{l}\text { Hematological } \\
\text { malignancies }\end{array}$ & Pop-PK & $\begin{array}{l}\mathrm{CL}_{\mathrm{M}}[\mathrm{L} / \mathrm{day}]=55.7, \mathrm{Q} 5[\mathrm{~L} / \mathrm{day}]=65.0, \mathrm{BW}: 0.75 \text { fixed exponent, } \\
\mathrm{V}[\mathrm{L}]=79.8, \mathrm{~V} 5[\mathrm{~L}]=28.1, \mathrm{BW}: 1 \text { fixed exponent }\end{array}$ & $\begin{array}{l}\text { Investigational, Dose, } \\
\text { Covariates }\end{array}$ & $\underline{\underline{\text { Li et al.. }}}$ \\
\hline \multirow[t]{3}{*}{ Busulfan } & $\begin{array}{l}\text { Hematopoietic cell } \\
\text { transplant }\end{array}$ & Pop-PK & & Pediatrics, Dose & $\begin{array}{l}\text { (McCune et } \\
\text { al.., 2014) }\end{array}$ \\
\hline & $\begin{array}{l}\text { Hematopoietic cell } \\
\text { transplant }\end{array}$ & Bayesian Pop-PK & $\begin{array}{l}\mathrm{k}_{\mathrm{el}} \text { : IdealBodyWeight, Age, } \\
\mathrm{V}: \text { IdealBodyWeight, Age }\end{array}$ & $\begin{array}{l}\text { Pediatrics, Limited sampling, } \\
\text { Covariates }\end{array}$ & (Neely et al.. \\
\hline & N/A & PBPK & & Pediatrics & (Diestelhorst \\
\hline
\end{tabular}


Pharmacokinetic models for precision dosing in oncology - Supplementary appendix

\begin{tabular}{|c|c|c|c|c|c|}
\hline & & & & & \\
\hline & $\begin{array}{l}\text { Hematopoietic cell } \\
\text { transplant }\end{array}$ & Pop-PK & $\begin{array}{l}\mathrm{CL}[\mathrm{L} / \mathrm{h} / 20 \mathrm{~kg}]=3.03, B W: 0.742 \text { (power), } \\
\mathrm{V}_{\mathrm{d}}[\mathrm{L} / \mathrm{h} / 20 \mathrm{~kg}]=12.8, B W: 0.843 \text { (power) }\end{array}$ & Pediatrics, Dose, Covariates & $\begin{array}{l}\text { (Booth et al.. } \\
\underline{2007)}\end{array}$ \\
\hline & $\begin{array}{l}\text { Hematopoietic cell } \\
\text { transplant }\end{array}$ & Pop-PK & CL: Leukemia & Pediatrics, Covariates & $\begin{array}{l}\text { (Hassan et al.. } \\
\underline{1996)}\end{array}$ \\
\hline & $\begin{array}{l}\text { Hematopoietic cell } \\
\text { transplant }\end{array}$ & Bayesian pop-PK & CL: $B S A$ & Limited sampling, Covariates & $\begin{array}{l}\text { (Nguyen et al., } \\
\underline{2006)}\end{array}$ \\
\hline & $\begin{array}{l}\text { Hematopoietic cell } \\
\text { transplant }\end{array}$ & Bayesian pop-PK & $\begin{array}{l}\mathrm{CL} / \mathrm{F}[\mathrm{L} / \mathrm{h}]=2.63 \cdot(\text { Age } / 18)^{0.376} \\
\cdot(\text { AsparatateAminotransferase } / 29)^{-0.161} \cdot 0.787^{\text {CancerType }} \\
\mathrm{V} / \mathrm{F}[\mathrm{L}]=9.26 \cdot(1+0.0734 \cdot(B W-10)) \\
\mathrm{k}_{\mathrm{a}}[\mathrm{h}=1]=1.26 \cdot(\text { Dose } / 1.0)-1.15 \cdot 2.49^{\text {Forrulation }}\end{array}$ & Pediatrics, Dose, Covariates & $\begin{array}{l}\text { (Nakamura et } \\
\text { al.. 2008) }\end{array}$ \\
\hline & Cancer (N/S) & Pop-PK & CL: $B S A, B W$ & Pediatrics, Dose, Covariates & $\begin{array}{l}\text { (Trame et al.. } \\
\text { 2011) } \\
\end{array}$ \\
\hline & $\begin{array}{l}\text { Hematopoietic cell } \\
\text { transplant }\end{array}$ & Pop-PK & $\begin{array}{l}\text { CL: } B W, \text { Day, } \\
\mathrm{V}_{1}: B W\end{array}$ & Pediatrics, Dose, Covariates & $\begin{array}{l}\text { (Bartelink et } \\
\text { al., 2012a) }\end{array}$ \\
\hline & Mixed disease & Pop-PK & & $\begin{array}{l}\text { Evaluation/validation, } \\
\text { Pediatrics }\end{array}$ & $\begin{array}{l}\text { (Bartelink et } \\
\text { al.., 2012b) } \\
\end{array}$ \\
\hline & $\begin{array}{l}\text { Hematopoietic cell } \\
\text { transplant }\end{array}$ & PK & & $\begin{array}{l}\text { Evaluation/validation, } \\
\text { Pediatrics }\end{array}$ & $\begin{array}{l}\text { (Abdel- } \\
\text { Rahman et al.. } \\
\text { 2016b) }\end{array}$ \\
\hline & $\begin{array}{l}\text { Hematopoietic cell } \\
\text { transplant }\end{array}$ & PK & & $\begin{array}{l}\text { Evaluation/validation, } \\
\text { Pediatrics }\end{array}$ & $\begin{array}{l}\text { (Abdel- } \\
\text { Rahman et al.. } \\
\text { 2016a) }\end{array}$ \\
\hline & $\begin{array}{l}\text { Hematopoietic cell } \\
\text { transplant }\end{array}$ & Bayesian pop-PK & CL: $B W, A g e$ & Pediatrics, Dose, Covariates & $\begin{array}{l}\text { (Long-Boyle } \\
\text { et al.. 2015) }\end{array}$ \\
\hline & $\begin{array}{l}\text { Hematopoietic cell } \\
\text { transplant }\end{array}$ & Pop-PK & $\begin{array}{l}\text { CL: AdjustedIBW, } A L T, \\
\text { V: } A \text { djustedIBW }\end{array}$ & $\begin{array}{l}\text { Covariates, Limited sampling, } \\
\text { Dose }\end{array}$ & $\begin{array}{l}\text { (de Castro et } \\
\underline{\text { al., 2015) }}\end{array}$ \\
\hline & $\begin{array}{l}\text { Hematopoietic cell } \\
\text { transplant }\end{array}$ & Pop-PK/PD & & $\begin{array}{l}\text { Covariates, Dose, Efficacy, } \\
\text { Toxicity, Pediatrics }\end{array}$ & $\begin{array}{l}\text { Bartelink et } \\
\text { al., 2016) }\end{array}$ \\
\hline & $\begin{array}{l}\text { Hematopoietic cell } \\
\text { transplant }\end{array}$ & $\begin{array}{l}\text { Pop-PK/Bayesian pop- } \\
\text { PK/PD (based dosing) }\end{array}$ & & $\begin{array}{l}\text { Pediatrics, Dose, } \\
\text { Evaluation/validation }\end{array}$ & $\begin{array}{l}\text { (Zao et al.. } \\
\text { 2015) } \\
\end{array}$ \\
\hline BYL719 & $\begin{array}{l}\text { Advanced solid } \\
\text { malignancies }\end{array}$ & Pop-PK/PD & & Efficacy, Dose & $\begin{array}{l}\text { (De Buck et } \\
\text { al.., 2014) }\end{array}$ \\
\hline Cabazitaxel & Advanced solid tumors & Pop-PK & $\mathrm{CL}[\mathrm{L} / \mathrm{h}]=48.6 \cdot B S A / 1.84 \cdot(1-0.536 \cdot$ BreastCancerTumourType $)$ & Covariates & $\begin{array}{l}\text { (Ferron et al.. } \\
2013) \\
\end{array}$ \\
\hline Cabozantinib & $\begin{array}{l}\text { Advanced malignancies, } \\
\text { recurrent/progressive } \\
\text { glioblastoma multiforme, } \\
\text { unresectable locally } \\
\text { advanced or metastatic } \\
\text { medullary thyroid cancer }\end{array}$ & Pop-PK & $\mathrm{CL} / \mathrm{F}[\mathrm{L} / \mathrm{day}]=106, B M I:-0.0244$, Sex: -0.219 & Dose, Covariates & $\begin{array}{l}\text { (Miles et al.. } \\
\text { 2016a) }\end{array}$ \\
\hline & Medullary thyroid cancer & Pop-PK/PD & $\begin{array}{l}\text { CL/F[L/day }]=108.5, \text { BMI: }-0.0276, \text { Sex: }-0.216 \text {, ParticipantPopulation: } \\
-0.408\end{array}$ & Efficacy, Covariates & $\begin{array}{l}\text { (Miles et al.. } \\
\underline{2016 \mathrm{~b}})\end{array}$ \\
\hline Capecitabine & Breast cancer, colorectal & Pop-PK & $\mathrm{k}_{\mathrm{a}}\left[\mathrm{h}^{-1}\right]=1.86 \cdot 0.45^{\text {AgeGroup }}$ & Other special populations & (Daher Abdi et \\
\hline
\end{tabular}


Pharmacokinetic models for precision dosing in oncology - Supplementary appendix

\begin{tabular}{|c|c|c|c|c|c|}
\hline & cancer & & $\mathrm{k}_{40}\left[\mathrm{~h}^{-1}\right]=77.1 \cdot 0.77^{\text {DayOrPKEvaluation }}$ & (Elderly), Covariates & al.. 2014) \\
\hline & Metastatic cancer & Pop-PK & $\begin{array}{l}\mathrm{CL}_{10}[\mathrm{~L} / \mathrm{h}]=218, \text { TotalBilirubin: } 0.32, \\
\mathrm{~K}_{34}\left[\mathrm{~h}^{-1}\right]=5.30 \text {, TotalBilirubin: }-0.36\end{array}$ & Metabolite, Covariates & $\begin{array}{l}\text { (Urien et al.. } \\
\underline{2005)}\end{array}$ \\
\hline & Breast cancer, other & Pop-PK & $\begin{array}{l}\text { CL of } 5^{\prime} \text {-DFUR }[\mathrm{L} / \mathrm{h}]=70.3, \text { Bilirubin: } 1.3, \text { BSA: } 1.1 \\
\text { CL of FBAL }[\mathrm{L} / \mathrm{h}]=22.7, C L_{C R}: 0.5\end{array}$ & Metabolite, Covariates & $\begin{array}{l}\text { (Gieschke et } \\
\text { al.., 2002) }\end{array}$ \\
\hline & Colorectal cancer & Pop-PK & $\begin{array}{l}\text { CL of } 5-\mathrm{FU}[\mathrm{L} / \mathrm{h}]=1190, A L P: 2, \\
\text { CL of FBAL }[\mathrm{L} / \mathrm{h}]=27.5, C L_{C R}: 0.5 \\
\mathrm{~V} \text { of FBAL }[\mathrm{L}]=72.6, C L_{C R}: 0.5, B S A: 1.3\end{array}$ & $\begin{array}{l}\text { Metabolite, Toxicity, } \\
\text { Covariates }\end{array}$ & $\begin{array}{l}\text { (Gieschke et } \\
\text { al., 2003) }\end{array}$ \\
\hline \multirow{9}{*}{ Carboplatin } & $\begin{array}{l}\text { Advanced non-small cell } \\
\text { lung cancer }\end{array}$ & Pop-PK & & Dose & $\begin{array}{l}\text { (Merino- } \\
\text { Sanjuan et al.. } \\
\text { 2011) }\end{array}$ \\
\hline & Advanced ovarian cancer & Pop-PK & $\begin{array}{l}\mathrm{CL}[\mathrm{L} / \mathrm{min}]=0.101+0.011 \cdot(B W-62.35)-0.0658 \cdot\left(S_{C R}-0.65\right) \\
\mathrm{V}_{1}[\mathrm{~L}]=15.5+0.163 \cdot(B W-62.35) \\
\mathrm{Q}[\mathrm{L} / \mathrm{min}]=0.0132-0.0103 \cdot(\text { Albumin }-3.65) \\
\mathrm{V}_{2}[\mathrm{~L}]=7.07-3.61 \cdot(\text { Albumin }-3.65)\end{array}$ & Limited sampling, Covariates & $\begin{array}{l}\text { (Shen et al.. } \\
\underline{\text { 2002) }}\end{array}$ \\
\hline & Ovarian cancer & Bayesian pop-PK & $\begin{array}{l}\mathrm{CL}[\mathrm{L} / \mathrm{h}]=0.15 \cdot L B W^{0.75}+0.78 \cdot C L_{C R} \\
\mathrm{~V}_{\mathrm{s}}[\mathrm{L}]=0.38 \cdot L B W \\
\mathrm{~V}_{1}[\mathrm{~L}]=0.26 \cdot L B W \\
\mathrm{CL}_{\mathrm{ic}}[\mathrm{L} / \mathrm{h}]=0.15 \cdot L B W^{0.75}\end{array}$ & Dose, Covariates & $\begin{array}{l}\text { (Duffull et al.. } \\
\text { 1997) }\end{array}$ \\
\hline & Germ cell cancer & Pop-PK/PD & $\begin{array}{l}\mathrm{CL}[\mathrm{mL} / \mathrm{min}]=110.0, C L_{C R, 24 h}: 0.408, \text { Height: } 1.05, \\
\mathrm{~V}_{\mathrm{c}}[\mathrm{L}]=19.8, B W: 0.091, \\
\mathrm{k}_{12}\left[\mathrm{~h}^{-1}\right]=0.042, B W: 04.7 \cdot 10^{-4}, \text { Height: } 7.5 \cdot 10^{-4}, \text { Age: }-6.4 \cdot 10^{-4}, C L_{C R, 24 h}: \\
-8.1 \cdot 10^{-5}\end{array}$ & Dose, Toxicity, Covariates & $\begin{array}{l}\text { (Kloft et al.. } \\
\underline{2003)}\end{array}$ \\
\hline & Mixed & Pop-PK & & Renal function & $\begin{array}{l}\text { (Ekhart et al., } \\
\text { 2006) }\end{array}$ \\
\hline & Cancer (N/S) & Bayesian pop-PK & & Pediatrics, Limited sampling & $\frac{\text { Peng et al.. }}{1995)}$ \\
\hline & Cancer (N/S) & Pop-PK & $\mathrm{CL}[\mathrm{mL} / \mathrm{min}]=114.2 \cdot\left(C L_{C R} / 103.1\right)^{0.34} \cdot(B W / 70)^{0.75} \cdot(1+$ InfusionDuration $)$ & Dose, Pediatrics, Covariates & $\begin{array}{l}\text { (Lindauer et } \\
\text { al., 2010b) }\end{array}$ \\
\hline & Metastatic germ cell cancer & Bayesian pop-PK & $\begin{array}{l}\text { CL: } B W, A g e, S e x, S_{C R} \\
\text { V1, V2: } B S A\end{array}$ & $\begin{array}{l}\text { Limited sampling, Dose, } \\
\text { Covariates }\end{array}$ & $\begin{array}{l}\text { (Chatelut et } \\
\text { al., 2000) }\end{array}$ \\
\hline & Mixed cancer & Bayesian pop-PK & & Dose, Evaluation/validation & $\begin{array}{l}\text { (Guillet et al.. } \\
\underline{1997)}\end{array}$ \\
\hline Carfilzomib & Multiple myeloma & Pop-PK & CL: $B S A$ & Covariates & $\begin{array}{l}\text { (Ou et al.. } \\
\text { 2017) }\end{array}$ \\
\hline Carlumab & Refractory tumors & Pop-PK/PD & & Efficacy, Dose & $\begin{array}{l}\text { (Fetterly et al.. } \\
\text { 2013) }\end{array}$ \\
\hline $\mathrm{CC} 49(\mathrm{mAb})$ & Cancer (N/S) & PBPK & & Inter-species extrapolation & $\begin{array}{l}\text { (Davda et al.. } \\
\text { 2008) }\end{array}$ \\
\hline Cediranib & Cancer (N/S) & Pop-PK & $\begin{array}{l}\mathrm{CL} / \mathrm{F}[\mathrm{L} / \mathrm{h}]=26.3 \cdot(\text { Age } / 59)^{-0.409} \cdot(\mathrm{BW} / 73)^{0.517}, \\
\mathrm{~V}_{\mathrm{c}} / \mathrm{F}[\mathrm{L}]=489 \cdot(\mathrm{BW} / 73)^{0.65}\end{array}$ & DDIs, Covariates & $\begin{array}{l}(\underline{\text { Li et al... }} \\
\underline{2017 \mathrm{~b})}\end{array}$ \\
\hline Ceritinib & Cancer (N/S) & Pop-PK & $\begin{array}{l}\mathrm{CL} / \mathrm{F}[\mathrm{L} / \mathrm{h}]=24.6, \text { BW: } 0.642, \text { BaselineAlbumin: }: 0.254, \text { Albumin: }: 0.285, \\
\text { BaselineAlanineTransaminase: }-0.0859 \text {, AlanineTransaminase: }-0.0792, \\
\mathrm{k}_{\text {out }}\left[\mathrm{h}^{-1}\right]=0.148, \text { Japanese }: 6.84\end{array}$ & $\begin{array}{l}\text { Hepatic impairment, } \\
\text { Covariates }\end{array}$ & $\begin{array}{l}\text { (Hong et al.. } \\
\underline{2017)}\end{array}$ \\
\hline Cetuximab & Metastatic colorectal & Pop-PK & $\theta_{\mathrm{i}}=\theta \cdot\left(\mathrm{COV}_{\mathrm{i}} / \mathrm{COV}_{\text {median }}\right)^{\beta C O V}$ & Covariates & $\begin{array}{l}\text { (Azzopardi et } \\
\text { al., 2011) }\end{array}$ \\
\hline
\end{tabular}


Pharmacokinetic models for precision dosing in oncology - Supplementary appendix

\begin{tabular}{|c|c|c|c|c|c|}
\hline & cancer & & $\begin{array}{l}\mathrm{CL}[\mathrm{L} / \text { day }]=0.497, \text { InitialSerumAlbumin: }-0.0244, \\
\mathrm{~V}_{1}[\mathrm{~L}]=2.96, B S A: 0.42, \\
\mathrm{~V}_{2}[\mathrm{~L}]=4.65, B S A: 0.56 \\
\mathrm{k}_{0}[\mathrm{mg} / \text { day }]=8.71, B S A: 1.58\end{array}$ & & \\
\hline & $\begin{array}{l}\text { Head and neck squamous } \\
\text { cell cancer }\end{array}$ & Pop-PK & $\begin{array}{l}\theta_{\mathrm{i}}=\theta \cdot\left(\mathrm{COV}_{\mathrm{i}} / \mathrm{COV}_{\text {median }}\right)^{\beta C O V} \\
\mathrm{CL}[\mathrm{L} / \text { day }]=3.18, \text { Chemotherapy: }-0.45 \\
\mathrm{~V}_{1}[\mathrm{~L}]=3.18, \text { BSA: } 1.28, \\
\mathrm{~V}_{2}[\mathrm{~L}]=5.40, \text { BSA: } 5.54 \\
\mathrm{k}_{0}[\mathrm{mg} / \text { day }]=6.72, \text { Chemotherapy: } 1.2\end{array}$ & Covariates & $\begin{array}{l}\text { (Pointreau et } \\
\text { al., 2016) }\end{array}$ \\
\hline $\begin{array}{l}\text { Chloroquinoxaline } \\
\text { sulphonamide }\end{array}$ & Non-small cell lung cancer & Pop-PK & & Dose & $\begin{array}{l}\text { (Miller et al.. } \\
\underline{1997)}\end{array}$ \\
\hline Cilengitide & Solid tumors & Pop-PK & & Pediatrics & $\begin{array}{l}\text { (Zuhlsdorf et } \\
\text { al.. 2014) }\end{array}$ \\
\hline \multirow{12}{*}{ Cisplatin } & Malignant solid tumors & Pop-PK & $\begin{array}{l}\mathrm{CL}[\mathrm{L} / \mathrm{h}]=5.17+26.3 \cdot(B S A-1.855) \\
\mathrm{V}[\mathrm{L}]=41.4+24.6 \cdot(B S A-1.855)\end{array}$ & Covariates & $\begin{array}{l}\text { (de Jongh et } \\
\text { al., 2004) }\end{array}$ \\
\hline & Ovarian cancer & PK & & Investigational & $\begin{array}{l}\text { (Reece et al.. } \\
\text { 1987) }\end{array}$ \\
\hline & $\begin{array}{l}\text { Lung, stomach, colon, } \\
\text { mediastinal tumor, } \\
\text { indistinct, cancer }\end{array}$ & Pop-PK/PD & $\begin{array}{l}\text { CL: } B S A, \text { DoseSchedule, } \\
\text { V: } B S A\end{array}$ & Toxicity, Covariates & $\begin{array}{l}\text { (Nagai et al.. } \\
1998)\end{array}$ \\
\hline & Testicular cancer & Bayesian pop-PK & & Dose & $\begin{array}{l}\text { (Salas et al.. } \\
\underline{2006)}\end{array}$ \\
\hline & Cancer (N/S) & Pop-PK & CL: $B S A$ & Metabolite, Covariates & $\begin{array}{l}\text { (Hanada et al.. } \\
\text { 2001) }\end{array}$ \\
\hline & Ovarian cancer & PK & & Investigational & $\begin{array}{l}\text { (Griffiths et } \\
\text { al., 1987) }\end{array}$ \\
\hline & Breast, ovarian cancer & PK & & Investigational & $\begin{array}{l}\text { (Fracasso et } \\
\text { al.. 1987) }\end{array}$ \\
\hline & Breast, ovarian cancer & PK & & Investigational & $\begin{array}{l}\text { (Leone et al.. } \\
\text { 1987) }\end{array}$ \\
\hline & $\begin{array}{l}\text { Ovarian, ovarian and } \\
\text { cervical cancer, small cell } \\
\text { lung cancer }\end{array}$ & PK & & $\begin{array}{l}\text { Other special populations } \\
\text { (elderly) }\end{array}$ & $\begin{array}{l}\text { (Bonetti et al.. } \\
\text { 1994) }\end{array}$ \\
\hline & $\begin{array}{l}\text { Advanced ovarian cancer } \\
\text { receiving chemotherapy }\end{array}$ & Bayesian pop-PK & & Investigational & $\begin{array}{l}\text { (Panteix et al.. } \\
\text { 2002) }\end{array}$ \\
\hline & $\begin{array}{l}\text { Advanced non-small cell } \\
\text { lung cancer }\end{array}$ & $\begin{array}{l}\text { Pop-PK (w. } \\
\text { gemcitabine) }\end{array}$ & & $\begin{array}{l}\text { Transporter genotype } \\
\text { (SLC28A1), Metabolic } \\
\text { genotype (GST, CDA, } \\
\text { deoxycytidine kinase), } \\
\text { Toxicity, Covariates } \\
\end{array}$ & $\begin{array}{l}\text { (Joerger et al.. } \\
\text { 2012) }\end{array}$ \\
\hline & Cancer (N/S) & PBPK & & Pediatrics & $\begin{array}{l}\text { (Evans et al.. } \\
\text { 1982a) }\end{array}$ \\
\hline CKD-602 & Cancer (N/S) & $\begin{array}{l}\text { Semi-mech. Pop- } \\
\text { PK/PD }\end{array}$ & & Toxicity & $\begin{array}{l}(\text { Wu et al.. } \\
\text { 2012a) }\end{array}$ \\
\hline Clofarabine & Acute leukemia & Pop-PK & $\mathrm{CL}[\mathrm{L} / \mathrm{h}]=32.8 \cdot(B W / 40)^{0.75}$ & Pediatrics, Covariates & (Bonate et al.. \\
\hline
\end{tabular}


Pharmacokinetic models for precision dosing in oncology - Supplementary appendix

\begin{tabular}{|c|c|c|c|c|c|}
\hline & & & $\begin{array}{l}\left.\mathrm{V} 1=115 \cdot(B W / 40)^{1.00} \cdot \text { WhiteBloodCellCount }^{\prime} 10 \cdot 103\right)^{0.128} \\
\mathrm{Q} 2=20.5 \cdot(B W / 40)^{0.75}, \\
\mathrm{~V} 2=94.5 \cdot(B W / 40)^{1.00}\end{array}$ & & 2004) \\
\hline Cobimetinib & Solid tumors & Pop-PK & $\begin{array}{l}\theta_{\mathrm{i}}=\theta \cdot\left(\mathrm{COV}_{\mathrm{i}} / \mathrm{COV}_{\text {reference }}\right)^{\beta \mathrm{COV}} \\
\mathrm{CL} / \mathrm{F}[\mathrm{L} / \text { day }]=322, \text { Age: }-0.217 \\
\mathrm{~V} 2 / \mathrm{F}[\mathrm{L}]=511, \mathrm{BW}:-0.217\end{array}$ & Covariates & $\begin{array}{l}\text { (Han et al... } \\
2015)\end{array}$ \\
\hline \multirow{5}{*}{ Crizotinib } & Cancer (N/S) & PBPK & Suggests no dose adjustment in renal mild/moderate renal impairment. & Renal impairment & $\begin{array}{l}\text { (Tan et al.. } \\
\text { 2017) }\end{array}$ \\
\hline & $\begin{array}{l}\text { Mainly advanced non- } \\
\text { small cell lung cancer }\end{array}$ & Pop-PK/PD & & Dose, Efficacy & $\begin{array}{l}\text { (Yamazaki et } \\
\text { al., 2012) }\end{array}$ \\
\hline & Non-small cell lung cancer & Pop-PK & $\begin{array}{l}\mathrm{CL} / \mathrm{F}[\mathrm{L} / \mathrm{h}]=136 \cdot(0.46 \cdot(\text { Day }-1) /(1.17+(\text { Day }-1))) \\
\cdot(1-0.23 \cdot \text { Asian }) \cdot(1-0.112 \cdot \text { Female }) \cdot(\text { BW/65 }) 0.20 \cdot\left(C_{C R} /\right. \\
91.6) 0.16 \cdot(\text { TotalBilirubin } / 0.41)-0.07 \\
\mathrm{~V} 2 / \mathrm{F}[\mathrm{L}]=3520 \cdot(1-0.23 \cdot \text { Asian }) \cdot(1-0.23 \cdot \text { Female })\end{array}$ & $\begin{array}{l}\text { Renal impairment, Hepatic } \\
\text { impairment, Covariates }\end{array}$ & $\begin{array}{l}\text { (Wang et al.. } \\
\underline{2016)}\end{array}$ \\
\hline & $\begin{array}{l}\text { Healthy volunteers, Cancer } \\
(\mathrm{N} / \mathrm{S})\end{array}$ & PBPK & & DDIs (CYP3A4) & $\begin{array}{l}\text { Yamazaki et } \\
\text { al.., 2015) } \\
\end{array}$ \\
\hline & $\begin{array}{l}\text { Healthy volunteers, Cancer } \\
(\mathrm{N} / \mathrm{S})\end{array}$ & PBPK & & DDIs (CYP3A4) & $\begin{array}{l}\text { (Mao et al.. } \\
\underline{2013)}\end{array}$ \\
\hline Custirsen & Mixed cancer & Pop-PK & $\begin{array}{l}\theta_{\mathrm{i}}=\theta \cdot\left(\mathrm{COV}_{\mathrm{i}} / \mathrm{COV}_{\text {reference }}\right)^{\beta C O V} \\
\mathrm{CL}[\mathrm{L} / \mathrm{h}]=2.36, \text { Age: }-0.190, B W: 0.355, S_{C R}:-0.159, \\
\mathrm{Q} 3[\mathrm{~L} / \mathrm{h}]=0.0573, \text { Age: } 0.224\end{array}$ & Covariates & $\begin{array}{l}\text { (Edwards et } \\
\text { al., 2017) }\end{array}$ \\
\hline \multirow[t]{11}{*}{ Cyclophosphamide } & Neuroblastoma & Pop-PK & & Pediatrics & $\begin{array}{l}\text { (McCune et } \\
\text { al.. 2009b) } \\
\end{array}$ \\
\hline & $\begin{array}{l}\text { Hematopoietic cell } \\
\text { transplant }\end{array}$ & Bayesian pop-PK & & Dose & $\begin{array}{l}\text { (Salinger et } \\
\text { al., 2006) }\end{array}$ \\
\hline & $\begin{array}{l}\text { Hematopoietic cell } \\
\text { transplant }\end{array}$ & Pop-PK & & Dose & $\begin{array}{l}\text { (McDonald et } \\
\text { al., 2005) }\end{array}$ \\
\hline & Hematologic malignancy & Bayesian pop-PK & CL: Age & Dose, Covariates & $\begin{array}{l}\text { (McCune et } \\
\text { al.. 2009a) } \\
\end{array}$ \\
\hline & Hematologic malignancy & Pop-PK & $\begin{array}{l}\text { CL: CYP2B6 } \\
\text { V: } B W, \operatorname{Sex}\end{array}$ & $\begin{array}{l}\text { Metabolic genotype (CYP2B6, } \\
\text { CYP2C9, CYP2C19), } \\
\text { Covariates }\end{array}$ & $\begin{array}{l}(\text { Xie et al.. } \\
2006)\end{array}$ \\
\hline & Metastatic breast cancer & PK & & Investigational & $\begin{array}{l}\text { (Chen et al., } \\
\text { 1995) }\end{array}$ \\
\hline & Breast cancer & Semi-mech. Pop-PK & & Investigational & $\begin{array}{l}\text { (Hassan et al.. } \\
\text { 1999) }\end{array}$ \\
\hline & Cancer (N/S) & $\mathrm{PK} / \mathrm{PD}$ & & Efficacy, Dose & $\begin{array}{l}\text { (Gardner, } \\
\underline{2002)}\end{array}$ \\
\hline & Breast cancer & $\begin{array}{l}\text { Semi-mech. Pop- } \\
\text { PK/PD (4-hydroxy- } \\
\text { cyclophosphamide) }\end{array}$ & $\begin{array}{l}\mathrm{CL}[\mathrm{L} / \mathrm{h}]=255, \text { Albumin: } 2.61, \text { BSA: } 56.3 \\
\mathrm{~V} 1[\mathrm{~L}]=1970, A L T:-21.5, B W: 1.45 \\
\mathrm{~V} 2[\mathrm{~L}]=645, B W: 1.45\end{array}$ & Toxicity, Covariates & $\begin{array}{l}\text { (Sandstrom et } \\
\text { al., 2006) }\end{array}$ \\
\hline & Breast cancer & $\begin{array}{l}\text { Pop-PK (4-hydroxy- } \\
\text { cyclophosphamide) }\end{array}$ & & Investigational & $\begin{array}{l}\text { (Sandstrom et } \\
\text { al..1996) }\end{array}$ \\
\hline & High-risk/advanced breast & Semi-mech. Bayesian & & Metabolite, DDI (ThioTEPA) & $\begin{array}{l}\text { (Huitema et } \\
\text { al.. 2001) }\end{array}$ \\
\hline
\end{tabular}


Pharmacokinetic models for precision dosing in oncology - Supplementary appendix

\begin{tabular}{|c|c|c|c|c|c|}
\hline & $\begin{array}{l}\text { cancer, germ-cell cancer, } \\
\text { ovarian cancer }\end{array}$ & pop-PK & & & \\
\hline Cytarabine & Acute myeloid leukemia & Pop-PK & CL: BaselineWhiteBloodCellCount & Covariates & $\begin{array}{l}\text { (Krogh- } \\
\text { Madsen et al.. } \\
\text { 2012) } \\
\end{array}$ \\
\hline \multirow[b]{2}{*}{ Daunorubicin } & $\begin{array}{l}\text { Acute lymphoblastic } \\
\text { leukemia }\end{array}$ & Pop-PK & & Pediatrics & $\begin{array}{l}\text { (Hempel et al. } \\
\underline{2010)}\end{array}$ \\
\hline & Acute myeloid leukemia & Pop-PK & $\mathrm{V}_{\mathrm{c}}$ : BaselineWhiteBloodCellCount & Covariates & $\begin{array}{l}\text { (Krogh- } \\
\text { Madsen et al.. } \\
2012) \\
\end{array}$ \\
\hline \multirow{2}{*}{ Degarelix } & Healthy volunteers & Pop-PK/PD & & Efficacy & (Tornoe et al.. \\
\hline & Healthy volunteers & Pop-PK & & Investigational & $\begin{array}{l}\text { (Tornoe et al.. } \\
\text { 2004b) }\end{array}$ \\
\hline \multirow[b]{2}{*}{ Denosumab } & Cancer (N/S) & Pop-PK/PD & & Efficacy & $\frac{\text { (Perez Ruixo }}{\text { et al. 2015) }}$ \\
\hline & $\begin{array}{l}\text { Solid tumors with bone } \\
\text { metastases }\end{array}$ & Bayesian Pop-PK & $\begin{array}{l}\mathrm{CL}[\mathrm{mL} / \mathrm{h} / 66 \mathrm{~kg}]=3.25, \text { Hispanic/Caucasian: } 1.27, \\
\text { BreastCancer/Healthy: } 1.10, \text { ProstateCancer/Healthy: } 1.29, \\
\text { SolidTumours/Healthy: } 1.37, \text { GiantCellCancer/Healthy: } 1.19 \text {, } \\
\mathrm{V}_{1}[\mathrm{~mL} / 66 \mathrm{~kg}]=2620, \text { Black/Caucasian: } 0.769, \\
\mathrm{k}_{\mathrm{a}}\left[\mathrm{h}^{-1}\right]=0.0107, \text { Age power: }-0.509, \text { Reference: } 53.6\end{array}$ & Covariates & $\begin{array}{l}\text { (Gibiansky et } \\
\text { al., 2012) }\end{array}$ \\
\hline Diflomotecan & $\begin{array}{l}\text { Advanced malignant } \\
\text { tumors }\end{array}$ & $\begin{array}{l}\text { Semi-mech. Pop- } \\
\text { PK/PD }\end{array}$ & & Toxicity & $\begin{array}{l}\text { (Soto et al., } \\
\underline{\text { 2011a) }}\end{array}$ \\
\hline Dinutuxumab & Neuroblastoma & Pop-PK & & Investigational & $\frac{\text { (Marachelian }}{\text { et al. 2016) }}$ \\
\hline \multirow[t]{7}{*}{ Docetaxel } & Breast cancer & Pop-PK & $\mathrm{CL}[\mathrm{L} / \mathrm{h}]: 32.6$, Age: 1.24 & Sex, Covariates & $\begin{array}{l}\text { (Onoue et al.. } \\
\text { 2016) }\end{array}$ \\
\hline & Cancer $(\mathrm{N} / \mathrm{S})$ & PBPK, Pop-PK & & $\begin{array}{l}\text { Limited sampling, Dose, } \\
\text { Pediatrics }\end{array}$ & $\begin{array}{l}\text { (Thai et al.. } \\
\underline{2015)}\end{array}$ \\
\hline & Cancer (N/S) & Pop-PK & $\begin{array}{l}\mathrm{CL}[\mathrm{L} / \mathrm{h}]=43.8, \text { GestationalEffect: } 1.19, \\
\mathrm{Vc}[\mathrm{L}]=8.63, \text { GestationalEffect }: 1.07, \\
\mathrm{Vp} 1[\mathrm{~L}]=7.19, \text { GestationalEffect }: 1.37, \\
\mathrm{Vp} 2[\mathrm{~L}]=359, \text { GestationalEffect }: 0.903\end{array}$ & $\begin{array}{l}\text { Other special populations } \\
\text { (pregnancy), Covariates }\end{array}$ & $\begin{array}{l}\text { (van Hasselt et } \\
\text { al..2014) }\end{array}$ \\
\hline & Advanced cancer & Pop-PK & $\begin{array}{l}\mathrm{CL}_{\text {normal }}[\mathrm{L} / \mathrm{h}]=470, \\
\mathrm{CL}_{\text {impaired }}[\mathrm{L} / \mathrm{h}]=317, \\
\mathrm{CL}: A A G, \text { ErythromycinBreathTest }\end{array}$ & $\begin{array}{l}\text { Hepatic impairment, } \\
\text { Covariates }\end{array}$ & $\begin{array}{l}\text { (Hooker et al.. } \\
\underline{2008)}\end{array}$ \\
\hline & Solid tumors & Pop-PK/PD & CL: BSA, AAG, Albumin, Ethnicity, Sex & $\begin{array}{l}\text { Toxicity, Ethnicity, Metabolic } \\
\text { genotype (CYP3A), } \\
\text { Transporter genotype } \\
\text { (ABCB1), Covariates }\end{array}$ & $\begin{array}{l}\text { (Lewis et al.. } \\
\underline{2007)}\end{array}$ \\
\hline & Non-small cell lung cancer & Pop-PK/PD & $\begin{array}{l}\mathrm{CL}[\mathrm{L} / \mathrm{h}]=32.5, B W \text { normalized, Albumin, } \\
\text { V1 }[\mathrm{L}]=6.67, B W \text { normalized, } \\
\text { V2[L] }]=7.61, B W \text { normalized, } \\
\text { V3[L] }]=175, B W \text { normalized }\end{array}$ & Toxicity, Covariates & $\begin{array}{l}\text { (Fukae et al., } \\
\underline{2016)}\end{array}$ \\
\hline & Non-small cell lung cancer & Pop-PK/PD & CL: BSA: $38 \%$, Transaminases\&AlkalinePhosphatase: $27 \%, A A G:-19 \%$, & Toxicity, Covariates & (Bruno et al.. \\
\hline
\end{tabular}


Pharmacokinetic models for precision dosing in oncology - Supplementary appendix

\begin{tabular}{|c|c|c|c|c|c|}
\hline & & & Albumin: $-8 \%$, Elderly: $-7 \%$ & & 2001) \\
\hline & Advanced cancer & Pop-PK & $\mathrm{CL}[\mathrm{L} / \mathrm{h}]=21.51+217(1 /$ ErythromycinBreathTest_tmax $)-0.13 \cdot A L T$ & Covariates & $\begin{array}{l}\text { (Slaviero et } \\
\text { al.. 2004) }\end{array}$ \\
\hline & Mixed cancer & $\begin{array}{l}\text { Semi-mech. Pop- } \\
\text { PK/PD }\end{array}$ & & Toxicity, Covariates & $\begin{array}{l}\text { (Kloft et al.. } \\
\text { 2006) }\end{array}$ \\
\hline & Solid tumors & Pop-PK/PD & & Toxicity, Covariates & $\begin{array}{l}\text { (Ozawa et al., } \\
\text { 2007) }\end{array}$ \\
\hline & $\begin{array}{l}\text { Breast cancer, non-small } \\
\text { cell lung cancer, head and } \\
\text { neck cancer, other }\end{array}$ & Pop-PK & & Hepatic impairment, Dose & $\begin{array}{l}\text { (Minami et al.. } \\
\underline{\text { 2009) }}\end{array}$ \\
\hline & Metastatic breast cancer & $\begin{array}{l}\text { Semi-mech. Pop- } \\
\text { PK/PD }\end{array}$ & & Dose, Toxicity & $\begin{array}{l}\text { (Vainas et al.. } \\
\underline{2012)}\end{array}$ \\
\hline & Cancer (N/S) & Pop-PK & & DDIs (ritonavir) & $\begin{array}{l}\text { (Koolen et al.. } \\
\underline{2010)}\end{array}$ \\
\hline & Non-small cell lung cancer & $\begin{array}{l}\text { Pop-PK/PD (w. } \\
\text { cisplatin) }\end{array}$ & & $\begin{array}{l}\text { Other special populations } \\
\text { (elderly), Covariates, Toxicity }\end{array}$ & $\begin{array}{l}\text { (Minami et al.. } \\
\text { 2004) }\end{array}$ \\
\hline & Cancer (N/S) & $\mathrm{PK} / \mathrm{PD}$ (w. epirubicin) & & Toxicity, Dose & $\begin{array}{l}\text { (Meille et al.. } \\
\text { 2016) }\end{array}$ \\
\hline & Mixed cancer & Bayesian pop-PK & $\begin{array}{l}\mathrm{CL}[\mathrm{L} / \mathrm{h}]=B S A \cdot(22.1-3.55 \cdot A A G-0.095 \cdot A g e+0.225 \cdot \text { Albumin } \cdot(1- \\
0.334 \cdot \text { HepaticFunction })\end{array}$ & Covariates & $\begin{array}{l}\text { (Bruno et al.. } \\
\text { 1996) }\end{array}$ \\
\hline & Solid tumors & Pop-PK & $\begin{array}{l}\mathrm{CL}[\mathrm{L} / \mathrm{h}]=B S A \cdot\left(56.4 \cdot 1.05^{\text {HeterozzgousABCBI-CI236T }} \cdot 0.719^{\text {HomozygousABCBI- }}\right. \\
\left.{ }_{C l 236 T}+\theta_{10} \cdot A A G+\theta_{11} \cdot \text { Age }+\theta_{12} \cdot \text { Albumin }\right) \cdot(1-\text { HepaticFunction })\end{array}$ & $\begin{array}{l}\text { Transporter genotype } \\
\text { (ABCB1), Metabolic genotype } \\
\text { (CYP3A), Covariates }\end{array}$ & $\begin{array}{l}\text { (Bosch et al.. } \\
\underline{2006)}\end{array}$ \\
\hline & Cancer (N/S) & Pop-PK & $\mathrm{CL}[\mathrm{L} / \mathrm{h}]=B S A \cdot(34.5-0.254 \cdot A g e) / 35.6$ & Covariates & $\begin{array}{l}\text { (Launay- } \\
\text { Iliadis et al.. } \\
1995)\end{array}$ \\
\hline DOTATATE & Cancer (N/S) & PBPK & & Investigational & $\begin{array}{l}\text { (Kletting et al.. } \\
\text { 2012) }\end{array}$ \\
\hline Doxorubicin & Cancer (N/S) & Pop-PK & $\begin{array}{l}\mathrm{V}_{\mathrm{c}}[\mathrm{L}]=9.83, \text { GestationalEffect: } 1.23 \\
\mathrm{~V}_{\mathrm{pl}}[\mathrm{L}]=674 \text {. GestationalEffect }: 1.32\end{array}$ & $\begin{array}{l}\text { Other special populations } \\
\text { (pregnancy), Covariates }\end{array}$ & $\begin{array}{l}\text { (van Hasselt et } \\
\text { al.. 2014) }\end{array}$ \\
\hline & Breast cancer & Pop-PK/PD & $\mathrm{CL}[\mathrm{L} / \mathrm{h}]=47.6 \cdot(B S A / 1.8)^{1.4} \cdot(\text { ApartateTransaminase } / 21)^{-0.24} \cdot(A G E / 56)^{-0.54}$ & Toxicity. Covariates & $\begin{array}{l}\frac{\text { (Joerger et al.. }}{2007 c)} \\
200\end{array}$ \\
\hline & Cancer (N/S) & Pop-PK & $\begin{array}{l}\mathrm{CL}-B S A: 1.30 \text { (linear scaling), Age: } 0.286 \text { (power) } \\
\mathrm{V}_{1}, \mathrm{Q}_{2}, \mathrm{~V}_{2}, \mathrm{~V}_{3}, \mathrm{~V}_{3}, \mathrm{CL}_{\mathrm{M}}, \mathrm{V}_{\mathrm{M}}-B S A: 1.30 \text { (linear scaling) }\end{array}$ & Pediatrics, Covariates & $\begin{array}{l}\text { (Voller et al.. } \\
\underline{2015)}\end{array}$ \\
\hline & Advanced breast cancer & PK & & Investigational & $\begin{array}{l}\text { (Frenay et al.. } \\
\text { 1989) }\end{array}$ \\
\hline & Cancer (N/S) & $\mathrm{PK} / \mathrm{PD}$ & & Efficacy, Dose & $\begin{array}{l}\text { (Gardner. } \\
\text { 2002) }\end{array}$ \\
\hline & Mixed cancer & Pop-PK & & Pediatrics, Obesity, Covariates & $\begin{array}{l}\text { (Thompson et } \\
\text { al., 2009) }\end{array}$ \\
\hline & Breast cancer & Pop-PK & & Overweight, Dose & $\begin{array}{l}\text { (Barpe et al.. } \\
\text { 2010) }\end{array}$ \\
\hline & Breast cancer & Pop-PK & $\mathrm{CL}[\mathrm{L} / \mathrm{h}]=53.5$, Age: -0.393 (power) & Metabolite, Covariates & (Wong et al.. \\
\hline & Cancer (N/S) & Pop-PK & & Pediatrics, Metabolite & $\begin{array}{l}\text { (Kunarajah et } \\
\text { al., 2017) }\end{array}$ \\
\hline & Metastatic breast cancer & Pop-PK & & Toxicity, Covariates & (Xu et al.. \\
\hline
\end{tabular}


Pharmacokinetic models for precision dosing in oncology - Supplementary appendix

\begin{tabular}{|c|c|c|c|c|c|}
\hline & & & & & $2010)$ \\
\hline & Breast cancer & Pop-PK/PD & & $\begin{array}{l}\text { Metabolite, Toxicity, } \\
\text { Covariates }\end{array}$ & $\begin{array}{l}\text { (Liang et al.. } \\
\underline{\underline{2016}}\end{array}$ \\
\hline & Mixed cancer & PK & & Investigational & $\begin{array}{l}\text { (Rahman et } \\
\text { al., 1990) }\end{array}$ \\
\hline & Cancer (N/S) & PBPK & & Inter-species extrapolation & $\begin{array}{l}\text { Harris and } \\
\text { Gross, 1975) }\end{array}$ \\
\hline & Solid tumors & PBPK & & Investigational & $\begin{array}{l}\text { (Chan et al.. } \\
\text { 1978) }\end{array}$ \\
\hline E7080 & Advanced malignancies & Pop-PK/PD & & Toxicity & $\frac{\text { Keizer et al.. }}{2010)}$ \\
\hline E7820 & $\begin{array}{l}\text { Malignant solid tumors, } \\
\text { lymphoma }\end{array}$ & Pop-PK & & Food effects & $\begin{array}{l}\text { (Keizer et al.. } \\
\underline{\text { 2009) }}\end{array}$ \\
\hline Elotuzumab & $\begin{array}{l}\text { Relapsed/refractory } \\
\text { multiple myeloma }\end{array}$ & Pop-PK & $\begin{array}{l}\theta_{\mathrm{i}}=\theta \cdot\left(\mathrm{COV}_{\mathrm{i}} / \mathrm{COV}_{\text {reference }}\right)^{\beta C O V} \\
\mathrm{CL}[\mathrm{L} / \text { day }]=0.0895, B W: 1.16, \text { Lenalidomide/dexamethasone: } 0.666, \\
\mathrm{~V}_{\mathrm{C}}[\mathrm{L}]=4.04, B W: 0.332, \text { Female: } 0.796, \text { Asian: } 0.861,2- \\
\text { microglubilin }>3.5: 1.13, \\
\mathrm{Q}[\mathrm{L} / \text { day }]=0.676, B W: 0.75, \\
\mathrm{~V}_{\mathrm{P}}[\mathrm{L}]=2.22, B W: 0.75, \\
\mathrm{~V}_{\max }[\mathrm{\mu g} / \mathrm{mL} / \text { day }]=9.21, \text { M-Protein }: 0.178\end{array}$ & DDIs, Covariates & $\begin{array}{l}\text { (Gibiansky et } \\
\text { al.. 2016) }\end{array}$ \\
\hline Enzalutamide & Healthy volunteers & PBPK & & DDIs & $\begin{array}{l}\text { (Narayanan et } \\
\text { al.. 2016) }\end{array}$ \\
\hline Epacadostat & $\begin{array}{l}\text { Advanced solid } \\
\text { malignancies }\end{array}$ & Pop-PK/PD & $\begin{array}{l}\mathrm{CL} / \mathrm{F}[\mathrm{L} / \mathrm{h}]=49.3 \cdot(\mathrm{BW} / 83)^{0.90} \\
\mathrm{Vc} / \mathrm{F}[\mathrm{L}]=152 \cdot(\mathrm{BW} / 83)^{0.56}\end{array}$ & Efficacy, Covariates & $\begin{array}{l}\text { Shi et al... } \\
\text { 2017) }\end{array}$ \\
\hline \multirow{4}{*}{ Epirubicin } & Cancer (N/S) & Pop-PK & $\begin{array}{l}\mathrm{CL}[\mathrm{L} / \mathrm{h}]=83.4, \text { GestationalEffect }: 1.10 \\
\mathrm{~V}_{\mathrm{c}}[\mathrm{L}]=9.83, \text { GestationalEffect: } 1.55 \\
\mathrm{~V}_{\mathrm{pl} 1}[\mathrm{~L}]=10, \text { GestationalEffect: } 2.08\end{array}$ & $\begin{array}{l}\text { Other special populations } \\
\text { (pregnancy), Covariates }\end{array}$ & $\begin{array}{l}\text { (van Hasselt et } \\
\text { al.. 2014) }\end{array}$ \\
\hline & Metastatic breast cancer & PK & & Investigational & $\begin{array}{l}\text { (Jakobsen et } \\
\text { al., 1991) } \\
\end{array}$ \\
\hline & Breast cancer & $\begin{array}{l}\text { Semi-mech. Pop- } \\
\text { PK/PD }\end{array}$ & $\mathrm{CL}[\mathrm{L} / \mathrm{h}]=71.7$, Albumin: 1.33 , Bilirubin: -0.575 & Toxicity, Covariates & $\begin{array}{l}\text { (Sandstrom et } \\
\text { al., 2006) }\end{array}$ \\
\hline & Breast cancer & Pop-PK & & Investigational & $\begin{array}{l}\text { (Sandstrom et } \\
\text { al.. 1996) }\end{array}$ \\
\hline Eribulin mesylate & $\begin{array}{l}\text { Breast cancer, prostate } \\
\text { cancer, solid tumors, non- } \\
\text { small cell lung cancer }\end{array}$ & $\begin{array}{l}\text { Semi-mech. Pop- } \\
\text { PK/PD }\end{array}$ & & Toxicity, Dose, Covariates & $\begin{array}{l}\text { (van Hasselt et } \\
\underline{\text { al., 2013) }}\end{array}$ \\
\hline \multirow[b]{2}{*}{ Erlotinib } & Cancer $(\mathrm{N} / \mathrm{S})$ & $\mathrm{PK} / \mathrm{PD}$ & & Investigational & $\begin{array}{l}\text { Foo et al.. } \\
\text { 2012) } \\
\end{array}$ \\
\hline & Non-small cell lung cancer & Pop-PK & $\begin{array}{l}\mathrm{CL}[\mathrm{mL} / \mathrm{h}]=43+0.356 \cdot C L_{C R} \\
\mathrm{~V}_{\mathrm{c}}-B W, S e x\end{array}$ & Toxicity, Covariates & $\begin{array}{l}\text { (Lu et al.., } \\
\underline{2006)}\end{array}$ \\
\hline Ewinia asparaginase & $\begin{array}{l}\text { Acute lymphoblastic } \\
\text { leukemia }\end{array}$ & Pop-PK & & Pediatrics & $\begin{array}{l}\underline{\text { Sassen et al.. }} \\
\underline{\underline{2017})}\end{array}$ \\
\hline \multirow[t]{2}{*}{ Etoposide } & $\begin{array}{l}\text { Non-Hodgkin's lymphoma, } \\
\text { Hodgkin's lymphoma }\end{array}$ & Pop-PK & $\mathrm{CL}[\mathrm{L} / \mathrm{h}]=0.0019 \cdot\left(C L_{C R} / 91.7\right)^{0.245} \cdot(\text { Bilirubin } / 7)^{-0.161}$ & Covariates & $\begin{array}{l}\text { (You et al.. } \\
\underline{2015} \text { ) }\end{array}$ \\
\hline & Acute leukemia & PK & & Pediatrics & $\begin{array}{l}\text { (Evans et al... } \\
\text { 1982b) }\end{array}$ \\
\hline
\end{tabular}


Pharmacokinetic models for precision dosing in oncology - Supplementary appendix

\begin{tabular}{|c|c|c|c|c|c|}
\hline & Mixed cancer & Bayesian pop-PK & & Limited sampling & $\begin{array}{l}\text { (Tranchand et } \\
\text { al.. 1999) }\end{array}$ \\
\hline & $\begin{array}{l}\text { Multifocal hepatocellular } \\
\text { carcinoma, advanced non- } \\
\text { small cell lung cancer, } \\
\text { gastric cancer, breast } \\
\text { cancer, other }\end{array}$ & Pop-PK/PD & $\begin{array}{l}\mathrm{CL}[\mathrm{L} / \mathrm{h}]=114, C L_{C R} \\
\mathrm{~V}[\mathrm{~L}]=6.0, B S A, \text { ProteinBinding, } \\
\mathrm{k}_{12}\left[\mathrm{~h}^{-1}\right]=0.14, \text { ProteinBinding, } \\
\mathrm{k}_{21}\left[\mathrm{~h}^{-1}\right]=0.06, C L_{C R}\end{array}$ & Efficacy, Toxicity, Covariates & $\begin{array}{l}\text { (Toffoli et al.. } \\
\text { 2001) }\end{array}$ \\
\hline & Mixed cancer & $\begin{array}{l}\text { Semi-mech. Pop- } \\
\text { PK/PD }\end{array}$ & & Toxicity, Covariates & $\begin{array}{l}\text { (Kloft et al., } \\
\underline{2006)}\end{array}$ \\
\hline & Small cell lung cancer & Pop-PK/PD & CL: $C L_{C R}$, Ifosfamide & Efficacy, Covariates & $\begin{array}{l}\text { (You et al... } \\
\text { 2008) }\end{array}$ \\
\hline & Metastatic breast cancer & PK & & Limited sampling & $\begin{array}{l}\text { (Millward et } \\
\text { al.. 1995) } \\
\end{array}$ \\
\hline & Acute myeloid leukemia & Pop-PK & $\begin{array}{l}\text { CL: BaselineWhiteBloodCellCount, } C L_{C R} \text {, } \\
\text { V: Sex }\end{array}$ & Covariates & $\begin{array}{l}\text { Krogh- } \\
\text { Madsen et al.. } \\
\underline{2012)}\end{array}$ \\
\hline & $\begin{array}{l}\text { Primary breast cancer, } \\
\text { Cancer (N/S) }\end{array}$ & PBPK & & Pediatrics & $\begin{array}{l}\text { (Kersting et } \\
\text { al., 2012) }\end{array}$ \\
\hline \multirow{4}{*}{ Everolimus } & $\begin{array}{l}\text { Progressive unresectable } \\
\text { recurrent or metastatic } \\
\text { thyroid cancer, metastatic } \\
\text { breast cancer }\end{array}$ & $\begin{array}{l}\text { Semi-mech. Pop- } \\
\text { PK/PD }\end{array}$ & & Efficacy & $\begin{array}{l}\text { (van Erp et al.. } \\
\underline{2016)}\end{array}$ \\
\hline & $\begin{array}{l}\text { Progressive, unresectable } \\
\text { or metastatic thyroid } \\
\text { cancer }\end{array}$ & Pop-PK & $\mathrm{F}=1$, TTThaplotideABCB1: 0.792 & $\begin{array}{l}\text { Transporter genotype } \\
\text { (ABCB1), Toxicity, Covariates }\end{array}$ & $\begin{array}{l}\text { (de Wit et al.. } \\
\underline{2016)}\end{array}$ \\
\hline & Solid tumors & PBPK-PD & & $\begin{array}{l}\text { Inter-species extrapolation, } \\
\text { Dose }\end{array}$ & $\begin{array}{l}\text { (Tanaka et al.. } \\
\underline{2008)}\end{array}$ \\
\hline & $\begin{array}{l}\text { Metastatic head and neck } \\
\text { squamous cell cancer }\end{array}$ & $\begin{array}{l}\text { Pop-PK (w. cetuximab } \\
\& \text { carboplatin) }\end{array}$ & & Investigational & $\begin{array}{l}\text { (Saba et al.. } \\
\underline{\underline{2014})} \\
\end{array}$ \\
\hline Exatecan mesylate & Metastatic gastric cancer & Pop-PK & $\mathrm{CL}, \mathrm{V}_{\mathrm{c}}, \mathrm{V}_{\mathrm{p}}, \mathrm{Q}, \mathrm{CL}-B S A$ & Covariates & $\begin{array}{l}\text { (Ajani et al.. } \\
\underline{2005)}\end{array}$ \\
\hline Farletuzumab & Epithelial ovarian cancer & Pop-PK & $\begin{array}{l}\theta_{\mathrm{i}}=\theta \cdot\left(\mathrm{COV}_{\mathrm{i}} / \mathrm{COV}_{\text {reference }}\right)^{\beta C O V} \\
\mathrm{CL}[\mathrm{L} / \mathrm{h}]=0.00784, B W: 0.715 \\
\mathrm{~V}_{\mathrm{c}}[\mathrm{L}]=3.00, B W: 0.629\end{array}$ & Covariates & $\begin{array}{l}\text { (Farrell et al.. } \\
\underline{2012)}\end{array}$ \\
\hline Foretinib & Cancer (N/S) & Pop-PK & $\begin{array}{l}\theta_{\mathrm{i}}=\theta \cdot\left(\mathrm{COV}_{\mathrm{i}} / \mathrm{COV}_{\text {reference }}\right)^{\beta C O V} \\
\mathrm{CL} / \mathrm{F}[\mathrm{L} / \mathrm{h}]=79.3, \text { Age }-0.381, \text { AspartateAminotransferase: }-0.217, \\
\mathrm{~V}_{\mathrm{c}} / \mathrm{F}[\mathrm{L}]=2150, \text { BW: } 0.661, \\
\mathrm{~F} 1 \text {-capsule: } 1.37, \mathrm{~F} 1 \text {-gablet }: 1.20\end{array}$ & Covariates & $\begin{array}{l}\text { (Singh et al.. } \\
\underline{2015)}\end{array}$ \\
\hline Ftorafur & Advanced breast cancer & Pop-PK & & Metabolite (5-fluorouracil) & $\begin{array}{l}\text { (Urien et al.. } \\
\text { 2003b) }\end{array}$ \\
\hline \multirow[b]{2}{*}{ Fulvestrant } & Advanced breast cancer & PK & & Dose & $\begin{array}{l}\text { (Robertson et } \\
\text { al., 2004) }\end{array}$ \\
\hline & $\begin{array}{l}\text { Postmenopausal women } \\
\text { with HER-positive } \\
\text { advanced breast cancer }\end{array}$ & Pop-PK & & Dose & $\begin{array}{l}\text { (McCormack } \\
\text { and Sapunar. } \\
\underline{2008)}\end{array}$ \\
\hline
\end{tabular}


Pharmacokinetic models for precision dosing in oncology - Supplementary appendix

\begin{tabular}{|c|c|c|c|c|c|}
\hline GDC-0917 & $\mathrm{N} / \mathrm{A}$ & Pop-PK & & Inter-species extrapolation & $\begin{array}{l}\text { (Wong et al.. } \\
\text { 2013) }\end{array}$ \\
\hline GDC-0980 & $\mathrm{N} / \mathrm{A}$ & $\mathrm{PK} / \mathrm{PD}$ & & Inter-species extrapolation & $\begin{array}{l}\text { (Salphati et al.. } \\
\text { 2012) }\end{array}$ \\
\hline \multirow[b]{2}{*}{ Gefitinib } & Malignant solid tumors & Pop-PK & CL/F: Midazolam clearance (CYP3A activity) & $\begin{array}{l}\text { Toxicity, Metabolic phenotype } \\
\text { (CYP3A), Covariates }\end{array}$ & $\begin{array}{l}\text { (Li et al... } \\
\underline{2006)}\end{array}$ \\
\hline & $\begin{array}{l}\text { Healthy volunteers, solid } \\
\text { malignant tumors of non- } \\
\text { small cell lung cancer, } \\
\text { colorectal cancer, head and } \\
\text { breast cancer }\end{array}$ & PBPK & & Inter-species extrapolation & 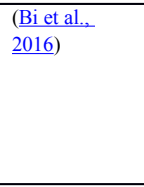 \\
\hline \multirow{6}{*}{ Gemcitabine } & Ovarian cancer & Pop-PK/PD & & Toxicity, Dose & $\begin{array}{l}\text { (Leijen et al.. } \\
\underline{2013)}\end{array}$ \\
\hline & Solid malignancies & Pop-PK & $\begin{array}{l}\mathrm{CLdFdu} / \mathrm{F}[\mathrm{L} / \mathrm{min}]=0.04 \cdot\left(1+0.48 \cdot C L_{C R} / 70\right) \\
\mathrm{CvdFdU} / \mathrm{F}[\mathrm{L}]=46 \cdot(B S A / 1.73) 0.93 \cdot 0.65^{\text {GemcitabineOxaliplatin }} \\
\cdot 0.54^{\text {OxaliplatinGemcitabine }} \cdot 1.24^{\text {NonSmallCellLungCancer }}\end{array}$ & $\begin{array}{l}\text { Metabolite, DDI (oxaliplatin), } \\
\text { Covariates }\end{array}$ & $\begin{array}{l}\text { (Jiang et al.. } \\
\underline{2008)}\end{array}$ \\
\hline & Urothelial cancer & PK & & $\begin{array}{l}\text { Metabolite, Renal } \\
\text { impairment/Other special } \\
\text { populations (renal replacement } \\
\text { therapy) }\end{array}$ & $\begin{array}{l}\text { (Masumori et } \\
\text { al., 2008) }\end{array}$ \\
\hline & $\begin{array}{l}\text { Pancreatic cancer, lung } \\
\text { cancer, methothelium } \\
\text { cancer }\end{array}$ & Pop-PK & $\begin{array}{l}\mathrm{CL}[\mathrm{L} / \mathrm{h}]=73.70 \cdot B S A \cdot(1-0.639 \cdot C D A * 3 \text { homo }) \cdot(1-0.171 \cdot \text { CDA } * 3 \text { hetero }) \\
\cdot(1+0.0749 \cdot \text { NumberOfCDA-31delC }) \cdot(1+0.191 \cdot \text { CoAdminOfS- } 1) \\
\mathrm{CL}_{\mathrm{m}}[\mathrm{L} / \mathrm{h}]=11.00 \cdot B S A \cdot(1-0.00855 \cdot(\text { Age- } 62.67)) \cdot\left(1-0.732 \cdot\left(S_{C R}-0.70\right)\right), \\
\mathrm{V}_{\mathrm{m} 1}[\mathrm{~L}]=15.00 \cdot \mathrm{BSA} \cdot(1-0.00806 \cdot(\text { Age- } 62.67)) \cdot(1+0.239 \cdot \text { Male })\end{array}$ & $\begin{array}{l}\text { Metabolic genotype (cytidine } \\
\text { deaminase, deoxyctidine), } \\
\text { Transporter genotype } \\
\text { (SLC29A1), Metabolite, } \\
\text { Covariates }\end{array}$ & $\begin{array}{l}\text { (Sugiyama et } \\
\text { al., 2010) }\end{array}$ \\
\hline & Non-small cell lung cancer & $\begin{array}{l}\text { Pop-PK/PD (w. } \\
\text { carboplatin) }\end{array}$ & & Efficacy, Dose & $\begin{array}{l}\text { (Tham et al.. } \\
\underline{2008)}\end{array}$ \\
\hline & $\begin{array}{l}\text { Advanced non-small cell } \\
\text { lung cancer }\end{array}$ & Pop-PK & CL: $C D A$ (cytidine deaminase) & $\begin{array}{l}\text { Transporter genotype } \\
\text { (SLC28A1), Metabolic } \\
\text { genotype (GST, CDA, } \\
\text { deoxycytidine kinase), } \\
\text { Toxicity, Metabolite, } \\
\text { Covariates }\end{array}$ & $\begin{array}{l}\text { (Joerger et al.. } \\
\underline{2012)}\end{array}$ \\
\hline Herceptin & Cancer (N/S) & $\mathrm{PK} / \mathrm{PD}$ & & Efficacy, Dose & $\begin{array}{ll}\text { (Gardner. } \\
2002)\end{array}$ \\
\hline HuCC49-Delta-CH2 & Colorectal cancer & Pop-PK & $\begin{array}{l}\text { CL, Q: BW: } 0.75 \text { (exponent), } \\
\mathrm{V}_{1}, \mathrm{~V}_{2}: \text { BW: } 1 \text { (exponent) }\end{array}$ & Covariates & $\begin{array}{l}\text { (Fang et al.. } \\
\underline{\text { 2007) }}\end{array}$ \\
\hline Hydroxyurea & $\begin{array}{l}\text { Lymphoma, brain tumors, } \\
\text { acute myelocytic leukemia, } \\
\text { lung cancer, melanoma, } \\
\text { renal-cell carcinoma }\end{array}$ & Pop-PK & & Investigational & $\begin{array}{l}\text { (Tracewell et } \\
\text { al., 1995) }\end{array}$ \\
\hline Hypoxoside & Lung cancer & Pop-PK & & Investigational & $\begin{array}{l}\text { (Albrecht et } \\
\text { al., 1995) }\end{array}$ \\
\hline
\end{tabular}


Pharmacokinetic models for precision dosing in oncology - Supplementary appendix

\begin{tabular}{|c|c|c|c|c|c|}
\hline \multirow[t]{2}{*}{ Ibrutinib } & B-cell malignancy & Pop-PK & $\begin{array}{l}\text { F1: PrandialState, } \\
\text { D1: PrandialState, Antacids, } \\
\text { V2/F, V3/F: BW }\end{array}$ & $\begin{array}{l}\text { DDIs, Biopharmaceutics } \\
\text { effects, Ethnicity, Covariates }\end{array}$ & $\begin{array}{l}\text { (Marostica et } \\
\text { al..2015) }\end{array}$ \\
\hline & Healthy volunteers & PBPK & & DDIs (CYP3A4) & $\begin{array}{l}\text { (de Zwart et } \\
\text { al., 2016) } \\
\end{array}$ \\
\hline Idarubicin & Leukemia & PK & & Pediatrics, Metabolite & $\begin{array}{l}\text { (Reid et al.. } \\
1990)\end{array}$ \\
\hline Idasanutlin & Cancer (N/S) & PBPK & & DDIs & $\begin{array}{l}\text { (Glenn et al.. } \\
2016)\end{array}$ \\
\hline Idelalisib & $\begin{array}{l}\text { Healthy volunteers, } \\
\text { hematologic malignancy }\end{array}$ & Pop-PK & $\begin{array}{l}\mathrm{CL}[\mathrm{L} / \mathrm{h}]-\text { Healthy: } 19.69, \text { Patient: } 14.88, \\
\mathrm{CL} / \mathrm{F}[\mathrm{L} / \mathrm{h}]=\mathrm{CL} / \mathrm{F}_{\mathrm{i}} \cdot\left(B W_{i} / B_{\text {reference }}\right)^{0.24} \\
\mathrm{Q} / \mathrm{F}[\mathrm{L} / \mathrm{h}]-\text { Healthy: } 7.846, \text { Patient: } 11.82, \\
\mathrm{~F} 1-\text { Dose: }-0.262\end{array}$ & Covariates & $\begin{array}{l}(\text { Jin et al., } \\
\underline{2016)}\end{array}$ \\
\hline Ifosfamide & $\begin{array}{l}\text { Resistant small cell lung } \\
\text { cancer }\end{array}$ & Pop-PK & & Metabolite, Investigational & $\begin{array}{l}\text { (Kerbusch et } \\
\text { al.. 2001) }\end{array}$ \\
\hline \multirow{8}{*}{ Imatinib } & $\begin{array}{l}\text { Gastrointestinal stromal } \\
\text { tumors }\end{array}$ & Bayesian pop-PK & & Investigational & $\begin{array}{l}\text { (Gotta et al.. } \\
\underline{2012)}\end{array}$ \\
\hline & $\begin{array}{l}\text { Chronic myeloid leukemia, } \\
\text { gastrointestinal stromal } \\
\text { tumors }\end{array}$ & Pop-PK & $\mathrm{V}_{\mathrm{d}}: A A G$ & Covariates & $\begin{array}{l}\text { (Widmer et al. } \\
\underline{2006)}\end{array}$ \\
\hline & Soft tissue and bone cancer & Pop-PK & & $\begin{array}{l}\text { Other special populations } \\
\text { (smoking) }\end{array}$ & $\begin{array}{l}\text { (van Erp et al., } \\
\underline{2008)}\end{array}$ \\
\hline & Hepatocellular cancer & $\mathrm{PK} / \mathrm{PD}$ & & Efficacy, Hepatic impairment & $\begin{array}{l}\text { (Treiber et al. } \\
\text { 2008) }\end{array}$ \\
\hline & $\begin{array}{l}\text { Solid tumors, } \\
\text { gastrointestinal stromal } \\
\text { tumors }\end{array}$ & Pop-PK & $\begin{array}{l}\mathrm{CL}[\mathrm{L} / \mathrm{h}]=7.29 \cdot(B W / 54)^{0.56} \cdot\left(A A G / 1.133^{-0.65} \cdot(\text { Albuminemia } / 38)^{0.66}\right. \\
\mathrm{V}[\mathrm{L}]=202 \cdot(B W / 54)^{0.79} \cdot(A A G / 1.13)^{-1.01} \\
\mathrm{CL}_{\text {metaboliteffm }}[\mathrm{L} / \mathrm{h}]=52.2 \cdot(B W / 54)^{-0.62} \cdot(A A G / 1.13)^{-0.81} \cdot 0.70^{\text {StudyDay }}\end{array}$ & $\begin{array}{l}\text { Pediatrics, Metabolic genotype } \\
\text { (CYP3A4/5), Transporter } \\
\text { genotype (ABCB1, ABCG2), } \\
\text { AAG genotype, Metabolite, } \\
\text { Covariates }\end{array}$ & $\begin{array}{l}\text { (Petain et al.. } \\
\text { 2008) }\end{array}$ \\
\hline & Chronic myeloid leukemia & Pop-PK & $\begin{array}{l}\mathrm{CL} / \mathrm{F}[\mathrm{L} / \mathrm{h}]=(13.8-3.81 \cdot \text { Occasion }) \cdot(\text { BW/80 })^{0.301} \\
\cdot(\text { Hemoglobin } / 13)^{0.897} \cdot(\text { WhiteBloodCellCount } / 16)^{-0.105}, \\
\mathrm{~V} / \mathrm{F}[\mathrm{L}]=(252-7.82 \cdot \text { Occasion }) \cdot(\text { BW/80 })^{0.405} \\
\cdot(\text { Hemoglobin } / 13)^{0.676} \cdot(\text { WhiteBloodCellCount } / 16)^{-0.070}\end{array}$ & Covariates & $\begin{array}{l}\text { (Schmidli et } \\
\text { al., 2005) }\end{array}$ \\
\hline & $\begin{array}{l}\text { Advanced gastrointestinal } \\
\text { stromal tumors }\end{array}$ & Pop-PK/PD & $\begin{array}{l}\mathrm{CL}[\mathrm{L} / \mathrm{h}]=7.97 \cdot(A A G / 1.15)-0.52 \\
\mathrm{CL}_{\mathrm{m} / \mathrm{fm}}[\mathrm{L} / \mathrm{h}]=58.6 \cdot(A A G / 1.15)-0.60 \cdot 0.55^{\text {Occasion }}\end{array}$ & $\begin{array}{l}\text { Metabolite, Toxicity, } \\
\text { Covariates }\end{array}$ & $\begin{array}{l}\text { (Delbaldo et } \\
\text { al.., 2006) }\end{array}$ \\
\hline & Mixed cancer & Bayesian pop-PK & $\begin{array}{l}\mathrm{CL} / \mathrm{F}[\mathrm{L} / \mathrm{h}]=10.8 \cdot(B W / 70)^{0.75}, \\
\mathrm{~V} / \mathrm{F}[\mathrm{L}]=284 \cdot(B W / 70), \\
\mathrm{CL}_{\text {metabolite }} / \mathrm{F}[\mathrm{L} / \mathrm{h}]=9.65 \cdot(B W / 70)^{0.75} \\
\mathrm{~V}_{1, \text { metabolite }} / \mathrm{F}[\mathrm{L}]=11.6 \cdot(B W / 70), \\
\mathrm{V}_{2, \text { metabolite }} / \mathrm{F}[\mathrm{L}]=256 \cdot(B W / 70), \\
\mathrm{Q}_{2, \text { metabolite }}[\mathrm{L} / \mathrm{h}]=2.9 \cdot(B W / 70)^{0.75}\end{array}$ & $\begin{array}{l}\text { Metabolite, Pediatrics, } \\
\text { Covariates }\end{array}$ & $\begin{array}{l}\text { (Menon- } \\
\text { Andersen et } \\
\underline{\text { al.. 2009) }}\end{array}$ \\
\hline Indicine N-oxide & Malignant solid tumors & PK & & Pediatrics & $\begin{array}{l}\text { (Ames et al.. } \\
\text { 1982) }\end{array}$ \\
\hline
\end{tabular}


Pharmacokinetic models for precision dosing in oncology - Supplementary appendix

\begin{tabular}{|c|c|c|c|c|c|}
\hline \multirow{3}{*}{ Indisulam } & Cancer (N/S) & $\begin{array}{l}\text { Semi-mech. Pop- } \\
\text { PK/PD }\end{array}$ & & Toxicity & $\begin{array}{l}\text { (Soto et al.. } \\
\text { 2011a) } \\
\end{array}$ \\
\hline & Cancer (N/S) & $\begin{array}{l}\text { Semi-mech. Pop-PK } \\
\text { (w. irinotecan, } \\
\text { capecitabine, } \\
\text { carboplatin) }\end{array}$ & $\begin{array}{l}\mathrm{CL}[\mathrm{L} / \mathrm{h}]=46, \text { Caucasian: } 3.36 \\
\mathrm{~V}_{\max }[\mathrm{mg} / \mathrm{L} / \mathrm{h}]=4.19, \text { BSA }: 2.29 \\
\mathrm{Q}_{\mathrm{IF}-\mathrm{TIS}}=1190, \text { Caucasian }: 2.26 \\
\mathrm{~B}_{\max -\mathrm{TIS}}=9.42, \text { Caucasian }: 0.70\end{array}$ & Covariates & $\begin{array}{l}\text { (Zandvliet et } \\
\text { al., 2006) }\end{array}$ \\
\hline & Mixed cancer & Pop-PK & Vmax: $C Y P 2 C 9 * 3 / 2, C Y P 2 C 19 * 3$ & $\begin{array}{l}\text { Metabolic genotype } \\
\text { (CYP2C9/19), Toxicity, Dose, } \\
\text { Covariates }\end{array}$ & $\begin{array}{l}\text { (Zandvliet et } \\
\text { al., 2007) }\end{array}$ \\
\hline $\begin{array}{l}\text { Interferone-Alpha, interferone- } \\
\text { Gamma }\end{array}$ & Healthy volunteers & Pop-PK/PD & & Response & $\begin{array}{l}\text { (Garcia-Garcia } \\
\text { et al.. 2016) } \\
\end{array}$ \\
\hline Iodine, ${ }^{131} \mathrm{I}$ & $\begin{array}{l}\text { Post-surgical thyroid } \\
\text { carcinoma }\end{array}$ & PK & & Renal impairment, Dose & $\begin{array}{l}\text { (Pahlka and } \\
\text { Sonnad, 2006) }\end{array}$ \\
\hline Ipilimumab & Advanced melanoma & Pop-PK & $\begin{array}{l}\theta_{\mathrm{i}}=\theta \cdot\left(\mathrm{COV}_{\mathrm{i}} / \mathrm{COV}_{\text {reference }}\right)^{\beta \mathrm{CCOV}} \\
\mathrm{CL}[\mathrm{L} / \mathrm{h}]=0.015, \mathrm{BW}: 0.642, \text { LactateDehydrogenase: } 1.13, \\
\mathrm{~V}[\mathrm{~L}]=4.15, \mathrm{BW}: 0.708\end{array}$ & Covariates & $\begin{array}{l}\text { (Feng et al.. } \\
\text { 2014) }\end{array}$ \\
\hline \multirow[t]{8}{*}{ Irinotecan } & $\begin{array}{l}\text { Bile-duct cancer, other } \\
\text { cancer }\end{array}$ & PBPK & & Physiology & $\begin{array}{l}\text { (Yoshida et al.. } \\
\underline{2013} \text { ) }\end{array}$ \\
\hline & Malignant solid tumors & Pop-PK/PD & Supports flat dosing (w/o BSA) & Toxicity, Dose & $\begin{array}{l}\text { (de Jong et al.. } \\
\underline{2004)}\end{array}$ \\
\hline & Malignant solid tumors & Pop-PK & & Investigational & $\begin{array}{l}(\underline{X i e} \text { et al.. } \\
\text { 2002) }\end{array}$ \\
\hline & Cancer (N/S) & PBPK & & $\begin{array}{l}\text { Toxicity, Metabolic genotype } \\
\text { (UGT1A1), Transporter } \\
\text { genotype (OATP1B1) }\end{array}$ & $\begin{array}{l}\text { (Toshimoto et } \\
\text { al... 2017) }\end{array}$ \\
\hline & Gastric cancer & Pop-PK & $\begin{array}{l}\text { CL4 }-A G E, \\
\mathrm{~V} 5-B S A \\
\mathrm{~V} 6-A G E \\
\mathrm{~V} 7-C L_{C R} \\
\end{array}$ & Covariates & $\begin{array}{l}\text { (Ahn et al.. } \\
2010)\end{array}$ \\
\hline & Malignant solid tumors & Bayesian pop-PK & & Limited sampling & $\begin{array}{l}\text { (Nakashima et } \\
\text { al., 1995) }\end{array}$ \\
\hline & Metastatic digestive cancer & Bayesian pop-PK & & $\begin{array}{l}\text { Limited sampling, Metabolite, } \\
\text { Covariates }\end{array}$ & $\begin{array}{l}\text { (Poujol et al.. } \\
\text { 2007) }\end{array}$ \\
\hline & Refractory solid tumors & Pop-PK & 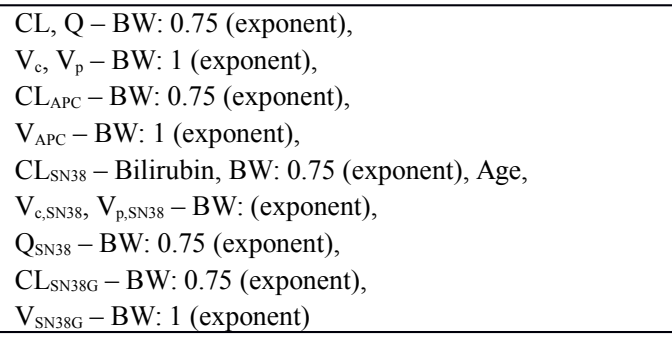 & $\begin{array}{l}\text { Pediatrics, Metabolite, } \\
\text { Covariates }\end{array}$ & $\begin{array}{l}\text { (Thompson et } \\
\text { al..2008) }\end{array}$ \\
\hline
\end{tabular}


Pharmacokinetic models for precision dosing in oncology - Supplementary appendix

\begin{tabular}{|c|c|c|c|c|c|}
\hline & Cancer (N/S) & PBPK & & Renal impairment, Metabolite & $\begin{array}{l}\text { (Fujita et al.. } \\
2016)\end{array}$ \\
\hline & $\begin{array}{l}\text { Glioblastoma, recurrent } \\
\text { glioma }\end{array}$ & Pop-PK & & DDIs, Metabolite & $\begin{array}{l}\text { Berg et al.. } \\
\underline{2015)}\end{array}$ \\
\hline Irofulven & Malignant cancer & Pop-PK & & Covariates & $\begin{array}{l}\text { (Urien et al.. } \\
\text { 2003a) }\end{array}$ \\
\hline Irosustat & Breast cancer & Pop-PK & & $\begin{array}{l}\text { Other special populations } \\
\text { (postmenopausal women) }\end{array}$ & $\begin{array}{l}\text { (Parra-Guillen } \\
\text { et al., 2015) }\end{array}$ \\
\hline Ixazomib & Cancer (N/S) & Pop-PK/PD & & Toxicity & $\begin{array}{l}\text { (Gupta et al.. } \\
\text { 2015) }\end{array}$ \\
\hline Kahalalide F & Malignant solid tumors & Pop-PK & & Covariates & $\begin{array}{l}\text { (Miguel-Lillo } \\
\text { et al.. 2015) }\end{array}$ \\
\hline \multirow[b]{2}{*}{ Lapatinib } & Healthy volunteers & PBPK & & Inter-species extrapolation & $\begin{array}{l}\text { (Hudachek } \\
\text { and Gustafson. } \\
\text { 2013) }\end{array}$ \\
\hline & $\begin{array}{l}\text { HER2-positive locally } \\
\text { advanced or metastatic } \\
\text { breast cancer }\end{array}$ & Pop-PK & & Dose, Covariates & $\begin{array}{l}\text { (Rezai et al.. } \\
\text { 2011) }\end{array}$ \\
\hline Lenvatinib & $\begin{array}{l}\text { Healthy volunteers, thyroid } \\
\text { cancer }\end{array}$ & Pop-PK & & Hepatic impairment, Dose & $\begin{array}{l}\text { (Gupta et al.. } \\
\underline{2016 \mathrm{~b})}\end{array}$ \\
\hline Letrozole & $\begin{array}{l}\text { Healthy volunteers } \\
\text { (postmenopausal women) }\end{array}$ & Pop-PK & $\begin{array}{l}\mathrm{CL} / \mathrm{F}[\mathrm{L} / \mathrm{h}]=1.03 \cdot(2 \text { CYP2A6alleles }+1 \text { CYP2A6allele } \\
\cdot 0.843+0 C Y P 2 A 6 \text { alleles } \cdot 0.448) \cdot\left(S_{C R} / 0.70\right)-1.27 \cdot(\text { AST } / 17.5)^{-0.793} \\
\mathrm{~V} / \mathrm{F}[\mathrm{L}]=94.2 \cdot(\mathrm{BW} / 51.1)^{1.12}\end{array}$ & $\begin{array}{l}\text { Metabolic genotype } \\
\text { (CYP2A6), Covariates }\end{array}$ & $\begin{array}{l}\text { (Tanii et al.. } \\
\underline{2011)}\end{array}$ \\
\hline Leuprolide & Prostate cancer & $\begin{array}{l}\text { Semi-mech. Pop- } \\
\text { PK/PD }\end{array}$ & & Efficacy, Covariates & $\begin{array}{l}\text { (Lim and } \\
\text { Salem, 2015) }\end{array}$ \\
\hline \multirow[t]{2}{*}{ Linifanib } & Mixed cancer & Pop-PK & $\begin{array}{l}\mathrm{CL} / \mathrm{F}[\mathrm{L} / \mathrm{h}]=2.82, \text { ColoRectalCancer }: 1.41, \text { Sex: } 0.75, \\
\mathrm{~V}_{\mathrm{c}} / \mathrm{F}[\mathrm{L}]=50.75, \text { BW: } 0.52, \text { HepatocellularCancer: } 1.63, \\
\text { RenalCellCancer: } 1.86, \\
\mathrm{~V}_{\mathrm{p}} / \mathrm{F}[\mathrm{L}]=10.36, B W: 0.52, \text { HepatocellularCancer: } 1.63, \\
\text { RenalCellCancer: } 1.86, \\
\mathrm{k}_{\mathrm{a}}[\mathrm{L} / \mathrm{h}]=0.46, \text { OralSolution: } 1.97, \\
\mathrm{~F}-\text { TimeOfDose: } 0.73, \text { FoodCondition: } 0.86, \\
\text { AcuteMyelogenousLeukemia/MyelodysplasticSyndrome: } 0.57\end{array}$ & Food effects, Covariates & $\begin{array}{l}\text { (Salem et al.. } \\
\underline{2014)}\end{array}$ \\
\hline & Hepatocellular cancer & Pop-PK & $\begin{array}{l}\text { CL/F [L/h] - Males: } 3.65, \text { Females: } 2.81 \text {, } \\
\text { V/F[L]-Males: } 96.8, \text { Females: } 71.5\end{array}$ & Dose, Covariates & $\begin{array}{l}\text { (Chiu et al.. } \\
\text { 2013) }\end{array}$ \\
\hline Lym-1, ${ }^{123} \mathrm{I}$ & B-cell lymphoma & Semi-mech. PK & & Investigational & $\begin{array}{l}\text { (Koizumi et } \\
\text { al.. 1986) }\end{array}$ \\
\hline \multirow{2}{*}{ Matizumab } & Advanced cancer & Pop-PK & $\begin{array}{l}\theta_{\mathrm{i}}=\theta \cdot\left(1+\beta \mathrm{COV} \cdot\left(\mathrm{COV}_{\mathrm{i}}-\mathrm{COV}_{\text {median }}\right)\right) \\
\mathrm{CL}_{\mathrm{L}}[\mathrm{mL} / \mathrm{h}]=15.3, \text { FatFreeMass: } 0.0138 \\
\mathrm{~V}_{1}[\mathrm{~L}]=3.79, \text { FatFreeMass: } 0.0077\end{array}$ & Dose, Covariates & $\begin{array}{l}\text { (Kuester et al.. } \\
\underline{\underline{K} 009)}\end{array}$ \\
\hline & Advanced cancer & Pop-PK & $\begin{array}{l}\theta_{\mathrm{i}}=\theta \cdot\left(1+\beta \mathrm{COV} \cdot\left(\mathrm{COV}_{\mathrm{i}}-\mathrm{COV}_{\text {median }}\right)\right) \\
\mathrm{CL}_{\mathrm{L}}[\mathrm{mL} / \mathrm{h}]=14.6, B W: 0.0087 \\
\mathrm{~V}_{\mathrm{I}}[\mathrm{L}]=3.73, B W: 0.0044\end{array}$ & Covariates & $\begin{array}{l}\text { (Kuester et al.. } \\
\underline{2008)}\end{array}$ \\
\hline Melphalan & Advanced malignancy & Bayesian pop-PK & $\mathrm{CL}[\mathrm{L} / \mathrm{h}]=1.37 \cdot C L_{C R}-18.9 \cdot(\operatorname{Sex}-1)+7.01$ & Covariates & $\begin{array}{l}\text { (Mougenot et } \\
\text { al.., 2004) }\end{array}$ \\
\hline
\end{tabular}


Pharmacokinetic models for precision dosing in oncology - Supplementary appendix

\begin{tabular}{|c|c|c|c|c|c|}
\hline & Advanced prostate cancer & Pop-PK/PD & $\begin{array}{l}\theta_{\mathrm{i}}=\theta \cdot\left(\mathrm{COV}_{\mathrm{i}} / \mathrm{COV}_{\text {reference }}\right)^{\beta C O V} \\
\mathrm{CL}[\mathrm{L} / \mathrm{h} / 70 \mathrm{~kg}]=10.9, B W: 0.0831, S_{C R}: 0.0831 \\
\mathrm{Q}[\mathrm{L} / \mathrm{h} / 70 \mathrm{~kg}]=0.110, B W: 0.693\end{array}$ & Toxicity, Covariates & $\begin{array}{l}\text { (Smith et al.. } \\
\underline{1993)}\end{array}$ \\
\hline & Mixed cancer & Bayesian pop-PK & $\begin{array}{l}\mathrm{CL}[\mathrm{L} / \mathrm{h}]=0+0.34 \cdot \mathrm{BW}-3.17 \cdot \text { PriorCarboplatinTherapy }+0.0377 \cdot \mathrm{GFR}, \\
\mathrm{V}[\mathrm{L}]=1.12+0.178 \cdot \mathrm{BW}\end{array}$ & Pediatrics, Dose, Covariates & $\begin{array}{l}\text { (Nath et al.. } \\
\underline{2007)}\end{array}$ \\
\hline & & Bayesian pop-PK & $\begin{array}{l}\mathrm{CL}_{\text {Non-renal }}[\mathrm{L} / \mathrm{h}]=79.7 \cdot(\text { Hematocrit } / 34)^{0.679} \cdot(\text { FatFreeMass } / 50)^{0.75} \\
\mathrm{CL}_{\text {Renal }}[\mathrm{L} / \mathrm{h}]=50.7 \cdot\left(\text { CL }_{C R} / 88\right) \\
\mathrm{V} 1[\mathrm{~L}]=63.8 \cdot(\text { FatFreeMass } / 50)\end{array}$ & Efficacy, Toxicity, Covariates & $\begin{array}{l}\text { (Nath et al.. } \\
\underline{2010)}\end{array}$ \\
\hline & Bone marrow transplant & PK & $\begin{array}{l}\mathrm{CL}[\mathrm{L} / \mathrm{h}]=4.77+0.37 \cdot \mathrm{BW}-3.99 \cdot \text { PriorCarboplatinTreatment- } \\
2.84 \cdot \mathrm{GFR}+2.10 \cdot \text { PriorTotalBodyIrradiation }\end{array}$ & Pediatrics, Dose, Covariates & $\begin{array}{l}\text { (Nath et al.. } \\
\underline{\text { 2005) }}\end{array}$ \\
\hline Menogaril & Metastatic tumors & PK & & Investigational & $\begin{array}{l}\text { (Piergies and } \\
\text { Benson, 1995) } \\
\end{array}$ \\
\hline \multirow{11}{*}{ Methotrexate } & Osteosarcoma & Pop-PK/PD & $\begin{array}{l}\theta_{\mathrm{i}}=\theta \cdot\left(\mathrm{COV}_{\mathrm{i}} / \mathrm{COV}_{\text {reference }}\right)^{\beta C O V} \\
\mathrm{CL}[\mathrm{L} / \mathrm{h}]=15.7, \text { Hematocrit: } 0.85 \\
\mathrm{Vc}[\mathrm{L}]=79.2, \text { BW: } 1.29\end{array}$ & Toxicity, Covariates & $\begin{array}{l}\text { (Johansson et } \\
\text { al., 2011) }\end{array}$ \\
\hline & Hematologic malignancy & Pop-PK & & Investigational & $\begin{array}{l}\text { (Nader et al.. } \\
\underline{2017)}\end{array}$ \\
\hline & Cancer (N/S) & $\mathrm{PK} / \mathrm{PD}$ & & Efficacy, Dose & $\begin{array}{l}\text { (Gardner. } \\
2002)\end{array}$ \\
\hline & Breast cancer & Bayesian pop-PK & & Dose & $\begin{array}{l}\text { Monjanel- } \\
\text { Mouterde et } \\
\text { al.. 2002) } \\
\end{array}$ \\
\hline & Cancer (N/S) & Bayesian pop-PK & & Dose, Pediatrics & $\begin{array}{l}\text { (Barrett et al.. } \\
\text { 2008) }\end{array}$ \\
\hline & Osteosarcoma & Pop-PK & $\begin{array}{l}\mathrm{CL}-C L_{C R}>89 \mu \mathrm{mol} / L, \text { AlanineAminotransferase, } \\
\mathrm{V}-\text { Height }, \text { BSA, Hemoglobin }\end{array}$ & Limited sampling, Covariates & $\begin{array}{l}\text { (Dupuis et al.. } \\
\underline{2008)}\end{array}$ \\
\hline & Cancer (N/S) & Pop-PK & & Covariates & $\begin{array}{l}\text { (Nagulu et al.. } \\
\underline{\underline{2010}}\end{array}$ \\
\hline & Cancer (N/S) & Pop-PK & $\begin{array}{l}\mathrm{CL}_{\mathrm{MTx}}[\mathrm{mL} / \mathrm{min}]=8.84+0.0423 \cdot\left(87-C L_{C R}\right)-2.45 \cdot \text { Benzimidazoles- } \\
1.46 \cdot N S A I D, \\
\mathrm{CL}_{\text {-oH-MTX }}[\mathrm{mL} / \mathrm{min}]=2+0.0423 \cdot\left(87-C L_{C R}\right)-2.45 \cdot \text { Benzimidazoles- } \\
0.369 \cdot \mathrm{NSAID}\end{array}$ & Metabolite, Covariates & $\begin{array}{l}\text { (Joerger et al.. } \\
\text { 2006a) }\end{array}$ \\
\hline & Mixed cancer & Bayesian pop-PK & & $\begin{array}{l}\text { Evaluation/validation, Dose, } \\
\text { Pediatrics }\end{array}$ & $\begin{array}{l}\text { (Dombrowsky } \\
\text { et al., 2011) }\end{array}$ \\
\hline & Osteosarcoma & Bayesian pop-PK & & Dose, Toxicity & $\begin{array}{l}\text { (Pignon et al.. } \\
\underline{1994)}\end{array}$ \\
\hline & Solid tumors & Bayesian pop-PK & & Dose, Evaluation/validation & $\begin{array}{l}\text { (Pignon et al., } \\
\underline{1995)}\end{array}$ \\
\hline Mitomycin C & Liver cancer & PK & & Investigational & $\begin{array}{l}\text { (Rump et al.. } \\
1996)\end{array}$ \\
\hline Mitoxantrone & $\begin{array}{l}\text { Metastatic breast cancer, } \\
\text { inflammatory breast cancer }\end{array}$ & PK & & Investigational & $\begin{array}{l}\text { (Richard et al.. } \\
\underline{1992)}\end{array}$ \\
\hline MNRP1685A & Advanced solid tumor & Pop-PK/PD & & Efficacy & (Xin et al... \\
\hline \multirow[t]{2}{*}{ Motesanib } & Thyroid cancer & Pop-PK/PD & & Efficacy, Dose & $\begin{array}{l}\text { (Claret et al.. } \\
\underline{2010)}\end{array}$ \\
\hline & Thyroid cancer & Pop-PK/PD & & Efficacy, Dose & (Luet al... \\
\hline
\end{tabular}


Pharmacokinetic models for precision dosing in oncology - Supplementary appendix

\begin{tabular}{|c|c|c|c|c|c|}
\hline & & & & & 2010) \\
\hline & Cancer (N/S) & Pop-PK & & Metabolite, Investigational & $\begin{array}{l}\text { (Gosselin et } \\
\text { al., 2015) } \\
\end{array}$ \\
\hline \multirow[b]{2}{*}{ Nab-paclitaxel } & Cancer (N/S) & Pop-PK & & Investigational & $\begin{array}{l}\text { (Chen et al.. } \\
\underline{2015)}\end{array}$ \\
\hline & Cancer (N/S) & Pop-PK/PD & $\mathrm{VM}_{\mathrm{EL}}[\mu \mathrm{g} / \mathrm{L}]=40.2$, Albumin: 0.341 & $\begin{array}{l}\text { Toxicity, Hepatic impairment, } \\
\text { Covariates }\end{array}$ & $\begin{array}{l}\text { (Chen et al.. } \\
\underline{2014)}\end{array}$ \\
\hline Navitoclax & Cancer (N/S) & $\begin{array}{l}\text { Semi-mech. Pop- } \\
\text { PK/PD }\end{array}$ & & Toxicity & $\begin{array}{l}\text { (Kaefer et al.. } \\
\text { 2014) }\end{array}$ \\
\hline Nimotuzumab & Advanced breast cancer & Semi-mech. Pop-PK & & Covariates & $\begin{array}{l}\text { (Rodriguez- } \\
\text { Vera et al.. } \\
\underline{2015)}\end{array}$ \\
\hline Nivolumab & Solid tumors & Pop-PK & $\begin{array}{l}\text { CL: } B W, e G F R, \text { BaselinePerformanceStatus, Sex, Asian, } \\
\mathrm{V}_{\mathrm{C}}: B W, S e x\end{array}$ & Covariates & $\begin{array}{l}\text { (Bajajet al.. } \\
\text { 2017) }\end{array}$ \\
\hline $\mathrm{O}(6)$-benzylguanine & $\begin{array}{l}\text { Central nervous system } \\
\text { tumors }\end{array}$ & PK & & Pediatrics & $\begin{array}{l}\text { (Neville et al.. } \\
\underline{2004)} \\
\end{array}$ \\
\hline Onartuzumab & Advanced solid tumors & Pop-PK & & $\begin{array}{l}\text { DDIs (erlotinib), Dose, } \\
\text { Covariates }\end{array}$ & $\begin{array}{l}(\text { Xin et al.. } \\
\text { 2013) }\end{array}$ \\
\hline Orteronel & Healthy volunteers & PBPK & & Renal impairment, DDIs & $\begin{array}{l}\text { (Lu et al... } \\
\text { 2014a) }\end{array}$ \\
\hline Osimertinib & Non-small cell lung cancer & Pop-PK & & Dose & $\begin{array}{l}\text { (Brown et al... } \\
\text { 2017) }\end{array}$ \\
\hline \multirow{6}{*}{ Oxaliplatin } & Peritoneal cancer & Pop-PK & $\begin{array}{l}\mathrm{V}_{\mathrm{II}}[\mathrm{L}]=3.73 \cdot(\text { BSA/1.69) } \\
\mathrm{k}_{\mathrm{EL}-\mathrm{B}}\left[\mathrm{h}^{-1}\right]=4.58 \cdot(\text { TotalProtein } / 42)^{-0.75}\end{array}$ & Surgery, Covariates & $\begin{array}{l}\text { Chalret du } \\
\text { Rieu et al.. } \\
\text { 2014b) } \\
\end{array}$ \\
\hline & Metastatic cancer & Pop-PK & $\begin{array}{l}\mathrm{CL}[\mathrm{L} / \mathrm{h}]=14.1 \cdot(B W / 71)^{1.10} \cdot\left(S_{C R} / 87\right)^{-0.57} \cdot(0.60 \text { if Female }) \\
\mathrm{V} 1[\mathrm{~L}]=24.9 \cdot(B W / 71)^{1.29} \\
\mathrm{Q}[\mathrm{L} / \mathrm{h}]=34.8 \cdot(B W / 71)^{1.01}\end{array}$ & Covariates & $\begin{array}{l}\text { (Bastian et al.. } \\
\underline{2003)}\end{array}$ \\
\hline & Cancer (N/S) & Pop-PK & $\begin{array}{l}\mathrm{CL}[\mathrm{L} / \mathrm{h}]=0.67 \cdot B W^{0.75} \cdot\left(0.6 / S_{C R}\right)^{0.67} \\
\mathrm{Q} 2[\mathrm{~L} / \mathrm{h}]=2.94 \cdot B W^{0.75}+\mathrm{Q} 3 \\
\mathrm{Q} 3[\mathrm{~L} / \mathrm{h}]=0.22 \cdot B W^{0.75} \\
\mathrm{~V} 1[\mathrm{~L}]=0.39 \cdot B W \cdot(A g e / 21)^{0.16} \\
\mathrm{~V} 2[\mathrm{~L}]=6.23 \cdot B W \\
\mathrm{~V} 3[\mathrm{~L}]=17.1 \cdot B W\end{array}$ & $\begin{array}{l}\text { Pediatrics, Renal impairment, } \\
\text { Hepatic impairment, } \\
\text { Covariates }\end{array}$ & $\begin{array}{l}\text { (Nikanjam et } \\
\text { al., 2015) }\end{array}$ \\
\hline & $\begin{array}{l}\text { Advanced colorectal } \\
\text { cancer }\end{array}$ & Pop-PK & $\mathrm{CL}[\mathrm{L} / \mathrm{h}]=0.28 \cdot B S A \cdot(1-0.058 \cdot$ Hematocrit $)$ & Covariates & $\begin{array}{l}\text { (Delord et al.. } \\
\underline{2003} \text { ) }\end{array}$ \\
\hline & Peritoneal carcinomatosis & $\begin{array}{l}\text { Semi-mech. Pop- } \\
\text { PK/PD }\end{array}$ & & Toxicity, Covariates & $\begin{array}{l}\text { (Valenzuela et } \\
\text { al.., 2011) }\end{array}$ \\
\hline & $\begin{array}{l}\text { Advanced colorectal } \\
\text { cancer }\end{array}$ & $\begin{array}{l}\text { Pop-PK (w. 5- } \\
\text { fluorouracil) }\end{array}$ & & Investigational, Covariates & $\begin{array}{l}\text { (Kho et al., } \\
\underline{\text { 2006) }}\end{array}$ \\
\hline \multirow[t]{2}{*}{ Paclitaxel } & Cancer (N/S) & Pop-PK/PD & & Efficacy, Formulation, Dose & $\begin{array}{l}\text { (Lim et al.. } \\
\text { 2015a) }\end{array}$ \\
\hline & Cancer (N/S) & Pop-PK & $\begin{array}{l}\mathrm{CL}[\mathrm{L} / \mathrm{h}]=4.71, \text { GestationalEffects: } 1.92, \\
\mathrm{Vc}[\mathrm{L}]=12.8, \text { GestationalEffects: } 4.21 \\
\mathrm{Vp} 1[\mathrm{~L}]=17.1, \text { GestationalEffects: } 3.16\end{array}$ & $\begin{array}{l}\text { Other special populations } \\
\text { (pregnancy), Covariates }\end{array}$ & $\begin{array}{l}\text { (van Hasselt et } \\
\text { al., 2014) }\end{array}$ \\
\hline
\end{tabular}


Pharmacokinetic models for precision dosing in oncology - Supplementary appendix

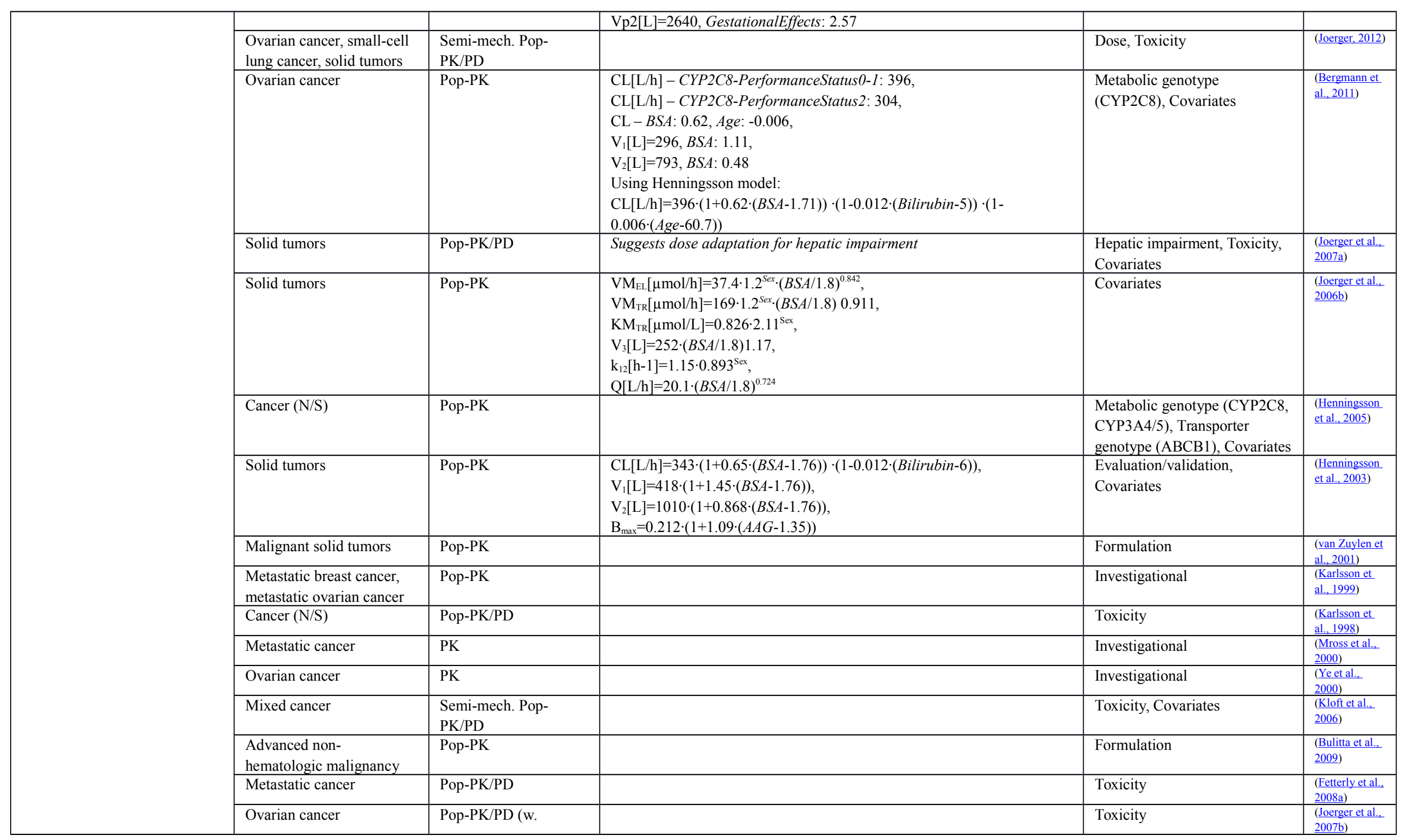


Pharmacokinetic models for precision dosing in oncology - Supplementary appendix

\begin{tabular}{|c|c|c|c|c|c|}
\hline & & carboplatin) & & & \\
\hline & Mixed cancer & $\begin{array}{l}\text { Semi-mech. Pop- } \\
\text { PK/PD }\end{array}$ & & Toxicity & $\begin{array}{l}\text { (Henningsson } \\
\text { et al., 2001) }\end{array}$ \\
\hline & $\begin{array}{l}\text { Relapsed or refractory } \\
\text { ovarian cancer, relapsed or } \\
\text { refractory breast cancer }\end{array}$ & Pop-PK/PD & & Toxicity, Efficacy & $\begin{array}{l}\text { (Gianni et al.. } \\
\text { 1995) }\end{array}$ \\
\hline Palbocicilib & $\begin{array}{l}\text { Advanced solid tumors, } \\
\text { non-Hodgkin lymphoma, } \\
\text { mantle cell lymphoma, } \\
\text { metastatic breast cancer }\end{array}$ & Pop-PK/PD & & Toxicity, Dose, Covariates & $\begin{array}{l}\text { (Sun et al.. } \\
\underline{2017)}\end{array}$ \\
\hline Panitumimab & Advanced solid tumors & Pop-PK & $\begin{array}{l}\mathrm{CL}[\mathrm{L} / \mathrm{day}]=0.273 \cdot(\mathrm{BW} / 80)^{0.411} \cdot 0.769^{\text {Female }} . \\
0.861^{\text {NonSmallCellLungCancer}} \cdot 0.957^{\text {RenalCancer }} \cdot 0.870^{\text {OtherCancer }}, \\
\mathrm{V}_{1}[\mathrm{~L}]=3.95 \cdot(\mathrm{BW} / 80)^{0.526} \cdot 0.831^{\text {Female }}, \\
\mathrm{V}_{\max }[\mathrm{mg} / \mathrm{day}]=12.1 \cdot(\mathrm{BW} / 80)^{0.621} \cdot(\text { Age } / 60)^{-0.495}\end{array}$ & Covariates & $\begin{array}{l}\text { (Ma et al.. } \\
\underline{2009)}\end{array}$ \\
\hline Patritumab & Non-small cell lung cancer & Pop-PK & & $\begin{array}{l}\text { Hepatic impairment, Renal } \\
\text { impairment }\end{array}$ & $\begin{array}{l}\text { (Yoshiba et al. } \\
\underline{2016)}\end{array}$ \\
\hline Patupilone & N/A & PBPK & & Inter-species extrapolation & $\begin{array}{l}\text { (Xia et al.. } \\
\text { 2012) }\end{array}$ \\
\hline \multirow{2}{*}{ Pazopanib } & Cancer (N/S) & Bayesian pop-PK/PD & & Toxicity & $\begin{array}{l}\text { (Suttle et al.. } \\
\underline{2013)}\end{array}$ \\
\hline & Cancer (N/S) & & & Investigational & $\begin{array}{l}\text { (Yuet al.. } \\
\underline{2017 \mathrm{a})} \\
\end{array}$ \\
\hline Pembrolizumab & Advanced solid tumors & Pop-PK & $\begin{array}{l}\mathrm{CL}[\mathrm{L} / \text { day }]=0.202 \cdot(B W / 76.8)^{0.578} \cdot(\text { Albumin } / 39.6)^{-} \\
\left.{ }_{0.854} \text { BaselineTumorBurden/89.6 }\right)^{0.0926 \cdot} \cdot(\text { eGFR/88.47 })^{0.139} \cdot((1-0.152) \text { if } \\
\text { Female }) \cdot(1+0.145) \text { if } \text { Non-small cell lung cancer }) \cdot((1+0.0739) \text { if Eastern } \\
\text { Cooperative Oncology Group }) \cdot((1+0.140) \text { if prior pilimumab }) \text {, } \\
\mathrm{V}[\mathrm{L}]=3.48 \cdot(\text { BW/76.8 })^{0.492} \cdot(\text { Albumin } / 39.6)^{-0.178} \cdot((1-0.134) \text { if } \text { Female }) \\
\cdot(1+0.0736) \text { if prior pilimumab })\end{array}$ & Dose, Covariates & $\begin{array}{l}\text { (Ahamadi et } \\
\text { al., 2017) }\end{array}$ \\
\hline \multirow{4}{*}{ Pemetrexed } & Mixed cancer & $\begin{array}{l}\text { Semi-mech. Pop- } \\
\text { PK/PD }\end{array}$ & & Toxicity, Covariates & $\begin{array}{ll}\text { Latz et al.. } \\
\text { 2006b) }\end{array}$ \\
\hline & Mixed cancer & Pop-PK & $\begin{array}{l}\mathrm{CL}[\mathrm{mL} / \mathrm{min}]=43.0+47.2 \cdot C L_{C R} / 92.6 \\
\mathrm{~V}_{1}[\mathrm{~L}]=6.13 \cdot B S A^{1.32}\end{array}$ & Covariates & $\begin{array}{l}\text { (Latz et al.. } \\
\text { 2006a) }\end{array}$ \\
\hline & $\begin{array}{l}\text { Metastatic colorectal } \\
\text { cancer, pancreatic cancer, } \\
\text { recurrent/metastatic breast } \\
\text { cancer, esophageal cancer }\end{array}$ & Pop-PK & $\begin{array}{l}\mathrm{CL}[\mathrm{L} / \mathrm{h}]=\left(2.82+0.0292 \cdot C L_{C R}+0.0475 \cdot(B W-70)+0.0041 \cdot(A L T-30.5)\right) \cdot(1- \\
0.344 \cdot F O L) \\
\mathrm{V}_{\mathrm{c}}[\mathrm{L}]=(11.3+0.105 \cdot(B W-70)) \cdot(1-0.325 \cdot \mathrm{Sex}) \\
\mathrm{V}_{\mathrm{p}}[\mathrm{L}]=9.78+4.28 \cdot(B S A-1.8)-1.25 \cdot(\text { Albumin }-3.67)\end{array}$ & Covariates & $\begin{array}{l}\text { (Ouellet et al.. } \\
\underline{2000)}\end{array}$ \\
\hline & Cancer (N/S) & PBPK & & DDIs & $\begin{array}{l}\text { (Posada et al.. } \\
\underline{2015)}\end{array}$ \\
\hline \multirow[t]{2}{*}{ Pertuzumab } & $\begin{array}{l}\text { Advanced solid } \\
\text { malignancies, advanced } \\
\text { ovarian cancer, metastatic } \\
\text { breast cancer }\end{array}$ & Pop-PK & $\begin{array}{l}\mathrm{CL}[\mathrm{L} / \text { day }]=0.214 \cdot(B W / 69)^{0.587} \cdot(\text { Albumin } / 39.2)^{-1.01} \cdot(A L K P / 107)^{0.169} \\
\mathrm{~V}_{c}[\mathrm{~L}]=2.74 \cdot(B S A / 1.72)^{1.160}\end{array}$ & Covariates & $\begin{array}{l}\text { (Ngg et al.. } \\
\underline{2006)}\end{array}$ \\
\hline & Neoadjuvant early breast & Pop-PK & & DDIs & (Quartino et \\
\hline
\end{tabular}


Pharmacokinetic models for precision dosing in oncology - Supplementary appendix

\begin{tabular}{|c|c|c|c|c|c|}
\hline & cancer & & & & al.., 2017) \\
\hline Phenylacetate & Refractory cancer & PK & & Pediatrics & $\begin{array}{l}\text { (Thompson et } \\
\text { al., 2003) }\end{array}$ \\
\hline PI-88 & Solid tumors & Pop-PK & $\begin{array}{l}\mathrm{CL}[\mathrm{mL} / \mathrm{h}]=3440+(1800 \cdot(\mathrm{BSA}-1.9))-(867 \cdot \text { Cancer }) \\
\mathrm{V}[\mathrm{mL}]=16900+(14400 \cdot(\mathrm{BSA}-1.9)) \\
\mathrm{k}_{\mathrm{a}}\left[\mathrm{h}^{-1}\right]=1.07-(0.0401 \cdot(\text { BMI-25.7)) }\end{array}$ & Covariates & $\begin{array}{l}\text { (Hudachek et } \\
\text { al..2010) }\end{array}$ \\
\hline Plitidepsin & $\begin{array}{l}\text { Advanced solid tumors, } \\
\text { advanced renal and } \\
\text { colorectal tumors, } \\
\text { advanced medullary } \\
\text { thyroid carcinoma, } \\
\text { advanced metastatic } \\
\text { melanoma }\end{array}$ & Pop-PK & & Covariates & $\begin{array}{l}\text { (Nalda-Molina } \\
\text { et al., 2009) }\end{array}$ \\
\hline PM00104 & Malignant tumors & Pop-PK/PD & & Toxicity, Covariates & $\begin{array}{l}\text { (Gonzalez- } \\
\text { Sales et al.. } \\
\underline{2012)}\end{array}$ \\
\hline Polyestradiol phosphate & Prostate cancer & PK & & Dose & $\begin{array}{l}\text { (Henriksson et } \\
\text { al., 1999) }\end{array}$ \\
\hline $\begin{array}{l}\text { Porfimer Sodium, 2-[1- } \\
\text { Hexyloxyelthyl]-2-devinyl } \\
\text { pyropheophorbide-a } \\
\text { (Photochlor), 5-ALA-induced } \\
\text { protoparophyrin IX }\end{array}$ & Cancer (N/S) & PK & & Investigational & $\begin{array}{l}\text { (Bellnier et al.. } \\
\underline{2006)}\end{array}$ \\
\hline Poziotinib & Advanced solid tumors & Pop-PK & $\begin{array}{l}\mathrm{k}_{\mathrm{a}}\left[\mathrm{h}^{-1}\right]=0.921, \mathrm{k}_{\mathrm{a}, \text { Food }}=0.359 \\
\mathrm{~V}_{\mathrm{c}} / \mathrm{F}[\mathrm{L}]=147 \cdot(B W / 70)^{1.23}\end{array}$ & Food effects, Covariates & $\begin{array}{l}\text { (Noh et al.. } \\
\underline{2015)}\end{array}$ \\
\hline PRO95780 & Cancer (N/S) & $\mathrm{PK} / \mathrm{PD}$ & & Inter-species extrapolation & $\begin{array}{l}\text { (Xiang et al.. } \\
\text { 2013) }\end{array}$ \\
\hline Pyrazine diazohydroxide & Solid tumor, lymphoma & PK & & Investigational & $\begin{array}{l}\text { (Vogelzang et } \\
\text { al., 1994) }\end{array}$ \\
\hline \multirow{2}{*}{ Raltitrexed } & Advanced solid tumors & Pop-PK & $\begin{array}{l}\mathrm{CL}[\mathrm{L} / \mathrm{h}]=0.54+0.02 \cdot C L_{C R} \\
\mathrm{~V}[\mathrm{~L}]=6.64+0.08 \cdot B W-0.16 \cdot \text { Albumin }\end{array}$ & Covariates & $\begin{array}{l}\text { (Blair et al.. } \\
\underline{2004)}\end{array}$ \\
\hline & $\begin{array}{l}\text { Recurrent or refractory } \\
\text { leukemia }\end{array}$ & PK & & Pediatrics & $\begin{array}{l}\text { (Horton et al.. } \\
\underline{2005)}\end{array}$ \\
\hline RG7160 & $\begin{array}{l}\text { Advanced EGFR-positive } \\
\text { solid tumors }\end{array}$ & Pop-PK & & Dose & $\begin{array}{l}\text { (Paz-Ares et } \\
\text { al., 2011) }\end{array}$ \\
\hline Rilotumumab & Mixed cancer & Pop-PK & $\begin{array}{l}\mathrm{CL}[\mathrm{L} / \text { day } / 70 \mathrm{~kg} / 60 \text { years }]=0.184, B W: 0.625 \text { (power) } \\
\text { Age: } 0.268 \text { (power), } \\
\mathrm{V}_{\mathrm{c}}[\mathrm{L} / 70 \mathrm{~kg} / 60 \text { years }]=3.56, B W: 0.611 \text { (power), Age: } 0.229 \text { (power) }\end{array}$ & Covariates & $\begin{array}{l}\text { (Zhu et al.. } \\
\text { 2014) }\end{array}$ \\
\hline Rituximab & $\begin{array}{l}\text { Diffuse large B-cell } \\
\text { lymphoma }\end{array}$ & Pop-PK & & Covariates & $\begin{array}{l}\text { (Tout et al.. } \\
\underline{2017)}\end{array}$ \\
\hline RO5323441 & $\begin{array}{l}\text { Advanced solid tumors, } \\
\text { metastatic treatment } \\
\text { refractory colorectal or } \\
\text { ovarian cancer, metastatic }\end{array}$ & Pop-PK & & DDIs & $\begin{array}{l}\text { (Wang et al.. } \\
\underline{2017)}\end{array}$ \\
\hline
\end{tabular}


Pharmacokinetic models for precision dosing in oncology - Supplementary appendix

\begin{tabular}{|c|c|c|c|c|c|}
\hline & $\begin{array}{l}\text { and/or unresectable } \\
\text { hepatocellular carcinoma }\end{array}$ & & & & \\
\hline Selumetinib & $\begin{array}{l}\text { Advanced solid tumors, } \\
\text { low-grade gliomas }\end{array}$ & Pop-PK & $\begin{array}{l}\text { F: FedState: } 0.117 \text { (linear coefficient), } \\
\text { D1[nmol/h] }=0.622, \text { FedState: } 4.09 \text { (linear coefficient for duration of zero } \\
\text { order input), } \\
\mathrm{t}_{\text {lag }}[\mathrm{h}]=0.319, \text { FedState: } 0.348 \text { (linear coefficient), } \\
\mathrm{CL} / \mathrm{F}[\mathrm{L} / \mathrm{h}]=13.5, B S A: 0.923 \text { (power), ALT: } 0.187 \text { (power), } \\
\mathrm{V}_{2} / \mathrm{F}[\mathrm{L}]=32.6, B S A: 1.24 \text { (power), Age: } 0.327 \text { (power) }\end{array}$ & Pediatrics, Dose, Covariates & $\begin{array}{l}\text { (Patel et al.. } \\
\text { 2017) }\end{array}$ \\
\hline Sepantropium bromide & $\begin{array}{l}\text { Advanced solid tumors, } \\
\text { low-grade glioma }\end{array}$ & Pop-PK & $\begin{array}{l}\mathrm{CL}[\mathrm{L} / \mathrm{h}]=42.1 \cdot\left(C L_{C R} / 79.22\right)^{0.425} \cdot(A L T / 19)^{0.124} \cdot 0.995^{\text {HormoneRefractory.ProstateCancer }} \\
\cdot 1.24^{\text {UnresectableMelanoma }}\end{array}$ & Covariates & $\begin{array}{l}\text { (Aoyama et } \\
\text { al.. 2013) }\end{array}$ \\
\hline Sibrotuzumab & $\begin{array}{l}\text { Advanced metastatic } \\
\text { cancer }\end{array}$ & Pop-PK & $\begin{array}{l}\theta_{\mathrm{i}}=\theta \cdot\left(1+\beta \mathrm{COV} \cdot\left(\mathrm{COV}_{\mathrm{i}}-\mathrm{COV}_{\text {median }}\right)\right) \\
\mathrm{CL}_{\mathrm{L}}[\mathrm{mL} / \mathrm{h}]=22.1, B W: 0.0182 \\
\mathrm{~V}_{1}[\mathrm{~L}]=4.13, B W: 0.0125 \\
\mathrm{~V}_{2}[\mathrm{~L}]=3.19, B W: 0.0105 \\
\mathrm{~V}_{\max }[\mathrm{mL} / \mathrm{h}]=0.0338, B W: 0.00934\end{array}$ & Covariates & $\begin{array}{l}\text { (Kloft et al., } \\
\text { 2004) }\end{array}$ \\
\hline \multirow[b]{2}{*}{ Sirolimus } & Healthy volunteers & PBPK & & Hepatic impairment, DDIs & $\begin{array}{l}\text { (Emoto et al.. } \\
\text { 2013) }\end{array}$ \\
\hline & Advanced cancer & Pop-PK & $\mathrm{CL} 1 / \mathrm{F}[\mathrm{L} / \mathrm{h}]=12.9 \cdot\left(\text { Hematocrit }_{\text {Median }} / \text { Hematocrit }_{i}\right)^{0.14}$ & Covariates & $\begin{array}{l}\text { (Wu et al.. } \\
2012 \mathrm{~b})\end{array}$ \\
\hline Sonidegib & Healthy volunteers & PBPK & & DDIs & $\begin{array}{l}\text { (Einolf et al.. } \\
\text { 2017) }\end{array}$ \\
\hline Sulindac & Healthy volunteers & Pop-PK & $\begin{array}{l}\mathrm{k}_{\mathrm{a}-\text {-Tablet }}[\mathrm{h}-1]=0.67, \mathrm{k}_{\mathrm{a}-\text {-Capsule }}[\mathrm{h}-1]=1.09, \\
\mathrm{~N}_{\text {Tablet }}=9.11, \mathrm{~N}_{\text {Capsule }}=15.5, \\
\mathrm{MTT}_{\text {Tablet }}[\mathrm{h}]=0.845, \operatorname{MTT}_{\text {Capsule }}[\mathrm{h}]=0.427, \\
\text { BIO }_{\text {Capsule }}=1.12\end{array}$ & Formulation, Covariates & $\begin{array}{l}\text { (Berg et al.. } \\
\underline{2013)}\end{array}$ \\
\hline \multirow{4}{*}{ Sunitinib } & $\begin{array}{l}\text { Advanced gastrointestinal } \\
\text { stromal tumor, advanced } \\
\text { renal cell carcinoma }\end{array}$ & Pop-PK/PD & & Efficacy, Toxicity, Covariates & $\begin{array}{l}\text { (Houk et al.. } \\
\underline{2010)}\end{array}$ \\
\hline & Cancer (N/S) & Semi-mech. Pop-PK & & Metabolite, Dose & $\begin{array}{l}\text { (Yu et al... } \\
\text { 2015) } \\
\end{array}$ \\
\hline & Acute myeloid leukemia & $\begin{array}{l}\text { Pop-PK (sunitinib } \\
\text { malate) }\end{array}$ & $\begin{array}{l}\text { Continuous: } \theta_{\mathrm{i}}=\theta \cdot\left(\mathrm{COV}_{\mathrm{i}} / \mathrm{COV}_{\text {reference }}\right)^{\beta C O V} \\
\text { Categorical: } \theta_{\mathrm{i}}=\theta \cdot(1+\beta \mathrm{COV} \cdot \mathrm{COV}) \\
\mathrm{CL} / \mathrm{F}[\mathrm{L} / \mathrm{h}]=51.8, \text { Sex: }-0.0876, \text { Asian: }-0.130, \text { GIST: }-0.285, \text { SolidTumor: } \\
-0.269, m R C C:-0.258 \\
\mathrm{~V} / \mathrm{F}[\mathrm{L}]=2030, B W: 0.459\end{array}$ & Covariates & $\begin{array}{l}\text { (Houk et al.. } \\
\text { 2009) }\end{array}$ \\
\hline & $\begin{array}{l}\text { Healthy volunteers, Cancer } \\
(\mathrm{N} / \mathrm{S})\end{array}$ & Pop-PK/PD & & Response, Covariates & $\begin{array}{l}\text { (Lindauer et } \\
\text { al., 2010a) }\end{array}$ \\
\hline \multirow{2}{*}{ Suramin } & Malignant cancer & Bayesian pop-PK & & Dose, Evaluation/validation & $\begin{array}{l}\text { (van Rijswijk } \\
\text { et al., 1992) }\end{array}$ \\
\hline & Prostate cancer & Bayesian pop-PK & & Dose, Evaluation/validation & $\begin{array}{l}\text { (Jodrell et al.. } \\
\text { 1994) }\end{array}$ \\
\hline \multirow[t]{2}{*}{ Tamoxifen } & Cancer (N/S) & $\mathrm{PK} / \mathrm{PD}$ & & Efficacy, Dose & $\begin{array}{l}\text { Gardner, } \\
\text { 2002) } \\
\end{array}$ \\
\hline & Breast cancer & Pop-PK & $\Theta 2 \mathrm{D} 6,1: 0.262, \Theta 3 \mathrm{~A} 4,1: 0.157$ & Metabolic phenotype (CYP3A, & $\begin{array}{l}\text { (ter Heine et } \\
\text { al., 2014) }\end{array}$ \\
\hline
\end{tabular}


Pharmacokinetic models for precision dosing in oncology - Supplementary appendix

\begin{tabular}{|c|c|c|c|c|c|}
\hline & & & & $\begin{array}{l}\text { CYP2D6), Metabolite, } \\
\text { Covariates }\end{array}$ & \\
\hline & Healthy volunteers & PBPK & & $\begin{array}{l}\text { Metabolite, Metabolic } \\
\text { genotype (CYP2D6), } \\
\text { Adherence }\end{array}$ & $\begin{array}{l}\text { (Dickschen et } \\
\text { al...2017) }\end{array}$ \\
\hline & Breast cancer & PBPK & & $\begin{array}{l}\text { Metabolic phenotype } \\
\text { (CYP2D6) }\end{array}$ & $\begin{array}{l}\text { (Dickschen et } \\
\text { al.., 2012) }\end{array}$ \\
\hline \multirow[b]{3}{*}{ Tegafur } & Gastric cancer & Pop-PK & & Surgery & $\begin{array}{l}(\text { Lim et al.. } \\
\text { 2015b) }\end{array}$ \\
\hline & Mixed cancer & $\begin{array}{l}\text { Bayesian pop-PK } \\
\text { (tegafur-potassium } \\
\text { oxonate-gimeracil) }\end{array}$ & $\begin{array}{l}\mathrm{V}_{\mathrm{FT}}[\mathrm{L}]=16.0 \cdot \mathrm{BSA}, \\
\mathrm{V}_{\text {5-FU }}[\mathrm{L}]=11.5 \cdot \mathrm{BSA}, \\
\mathrm{k}_{\mathrm{i}-\mathrm{Women}}[\mathrm{\mu g} / \mathrm{mL}]=0.037, \mathrm{k}_{\mathrm{i}-\mathrm{Men}[\mu \mathrm{g} / \mathrm{mL}]}=0.042\end{array}$ & Ethnicity, Covariates & $\begin{array}{l}\text { (Comets et al. } \\
\underline{\underline{2003)}}\end{array}$ \\
\hline & $\begin{array}{l}\text { Metastatic or recurrent } \\
\text { solid malignancy }\end{array}$ & $\begin{array}{l}\text { Pop-PK (tegafur-5- } \\
\text { fluorouracil) }\end{array}$ & $\begin{array}{l}\text { CDHP: } \\
\mathrm{CL} / \mathrm{F}[\mathrm{L} / \mathrm{h}]=\left(5.86+0.025 \cdot S_{C R}\right) \cdot B S A, \\
\mathrm{~V} / \mathrm{F}[\mathrm{L}]=42.7 \cdot B S A, \\
\mathrm{FT:} \\
\mathrm{k}_{\mathrm{a}}\left[\mathrm{h}^{-1}\right]=1.39, \mathrm{k}_{\mathrm{a}-\text { GastricResection }}\left[\mathrm{h}^{-1}\right]=1.62 \cdot 1.39, \\
\mathrm{CL} / \mathrm{F}_{\mathrm{CYP} 2 \mathrm{~A} \text {-No/oneVariantAllele }}[\mathrm{L} / \mathrm{h}]==2.02 \cdot B S A, \\
\mathrm{CL} / \mathrm{F}_{\mathrm{CYP} 2 \mathrm{AG}-\text {-TwoVariantAlleles }}[\mathrm{L} / \mathrm{h}]=0.58 \cdot 2.02 \cdot B S A\end{array}$ & $\begin{array}{l}\text { Metabolic genotype } \\
\text { (CYP2A6), Toxicity, Surgery, } \\
\text { Covariates }\end{array}$ & $\begin{array}{l}\text { (Hirose et al.. } \\
\underline{2010)}\end{array}$ \\
\hline \multirow[b]{2}{*}{ Temozolomide } & Advanced cancer & Pop-PK & CL: Sex, BSA, CancerType & Toxicity, Covariates & $\begin{array}{l}\text { (Jen et al.. } \\
2000)\end{array}$ \\
\hline & $\begin{array}{l}\text { Primary central nervous } \\
\text { system tumors }\end{array}$ & Pop-PK & $\mathrm{CL} / \mathrm{F}, \mathrm{V} / \mathrm{F}: B S A, A g e$ & $\begin{array}{l}\text { Pediatrics, Metabolite, } \\
\text { Covariates }\end{array}$ & $\begin{array}{l}\text { (Panetta et al.. } \\
\underline{2003)}\end{array}$ \\
\hline \multirow{2}{*}{ Temsirolimus } & Advanced renal cancer & Bayesian pop-PK & $\mathrm{CL}[\mathrm{L} / \mathrm{h}]=1.39$, BSA: 1.28, Dose: 0.551 & $\begin{array}{l}\text { Metabolite (sirolimus), } \\
\text { Toxicity, Efficacy, Covariates }\end{array}$ & $\begin{array}{l}\text { (Boni et al.. } \\
\underline{2005)}\end{array}$ \\
\hline & Recurrent solid tumors & Pop-PK & $\mathrm{CL}[\mathrm{L} / \mathrm{h} / 70 \mathrm{~kg}]: 3.71$, Dose: 0.878 & $\begin{array}{l}\text { Pediatrics, Metabolite, } \\
\text { Covariates }\end{array}$ & $\begin{array}{l}\text { (Mizuno et al.. } \\
\underline{2014)}\end{array}$ \\
\hline Teniposide & Acute leukemia & PK & & Pediatrics & $\begin{array}{l}\text { (Evans et al.. } \\
\text { 1982b) }\end{array}$ \\
\hline Thioguanine & Mixed cancer & PK & & Pediatrics & $\begin{array}{l}\text { (Kitchen et al.. } \\
\text { 1997) }\end{array}$ \\
\hline \multirow[t]{4}{*}{ Topotecan } & $\begin{array}{l}\text { Recurrent solid tumors, } \\
\text { recurrent acute leukemia, } \\
\text { medulloblastoma, high-risk } \\
\text { neuroblastoma, recurrent } \\
\text { Wilms tumor }\end{array}$ & Pop-PK & $\begin{array}{l}\mathrm{CL}\left[\mathrm{L} / \mathrm{h} / \mathrm{m}^{2}\right]=15.9+0.184 \cdot G F R+13.5 \cdot \text { MissingGFR+13.5 } \\
\cdot \text { TrialNo4+6.49'InvestigationFormulation- } 7.3 \cdot \text { Age }+9.9 \cdot \text { Phenytoin, } \\
\mathrm{V}\left[\mathrm{L} / \mathrm{m}^{2}\right]=32.7+6.0 \cdot \text { TrialNo1 }+15.3 \cdot \text { TrialNo4 }\end{array}$ & Pediatrics, Covariates & $\begin{array}{l}\text { (Schaiquevich } \\
\text { et al., 2007) }\end{array}$ \\
\hline & $\begin{array}{l}\text { Metastatic epithelial } \\
\text { ovarian cancer }\end{array}$ & Bayesian pop-PK & $\begin{array}{l}\mathrm{CL}[\mathrm{L} / \mathrm{h}]=5.47 \cdot C L_{C R}, \\
\mathrm{Vc}[\mathrm{L}]=0.584 \cdot B W\end{array}$ & Limited sampling, Covariates & $\begin{array}{l}\text { (Montazeri et } \\
\text { al..2000) }\end{array}$ \\
\hline & Refractory solid tumors & Pop-PK/PD & & Toxicity, Pediatrics & (Zamboni et \\
\hline & Solid tumors & Pop-PK & $\begin{array}{l}\mathrm{CL}[\mathrm{L} / \mathrm{h}]=\left(5.77+12.8 \cdot C L_{C R} / 70\right) \cdot(B W / 70)^{0.75} \cdot \exp (-0.120 \cdot \\
(\text { ECOGperformanceStatus }-1)) \\
\mathrm{V} 1[\mathrm{~L}]=19.3 \cdot(B W / 70)^{0.75}\end{array}$ & Toxicity, Covariates & $\begin{array}{l}\text { (Mould et al.. } \\
\text { 2002) }\end{array}$ \\
\hline
\end{tabular}


Pharmacokinetic models for precision dosing in oncology - Supplementary appendix

\begin{tabular}{|c|c|c|c|c|c|}
\hline & & & $\mathrm{V} 2[\mathrm{~L}]=45.7 \cdot(\mathrm{BW} / 70)$ & & \\
\hline & $\begin{array}{l}\text { Myelodysplastic } \\
\text { syndromes }\end{array}$ & Pop-PK/PD & $\mathrm{V}_{\mathrm{C}} \mathrm{F}[\mathrm{L}]=395, A g e$ & Dose, Covariates & $\begin{array}{l}\text { (Klein et al.. } \\
\underline{2006)}\end{array}$ \\
\hline & Mixed cancer & $\begin{array}{l}\text { Semi-mech. Pop- } \\
\text { PK/PD }\end{array}$ & & Toxicity, Covariates & $\begin{array}{l}\text { (Kloft et al.. } \\
\underline{\underline{2006})}\end{array}$ \\
\hline & $\begin{array}{l}\text { Metastatic epithelial } \\
\text { ovarian cancer }\end{array}$ & Bayesian pop-PK & & Limited sampling & $\begin{array}{l}\text { (Boucaud et } \\
\text { al., 2003) }\end{array}$ \\
\hline & $\begin{array}{l}\text { Metastatic epithelial } \\
\text { ovarian cancer }\end{array}$ & PK & & Evaluation/validation, Dose & $\begin{array}{l}\text { (Montazeri et } \\
\text { al.. 2002) }\end{array}$ \\
\hline \multirow{3}{*}{ Trabectedin } & Malignant tumors & $\begin{array}{l}\text { Semi-mech. Pop- } \\
\text { PK/PD }\end{array}$ & & Toxicity & $\begin{array}{l}\text { (Hing et al.. } \\
\underline{2008)}\end{array}$ \\
\hline & $\begin{array}{l}\text { Advanced solid tumors, } \\
\text { breast cancer, melanoma, } \\
\text { soft tissue sarcoma, renal } \\
\text { cancer, colorectal cancer, } \\
\text { ovarian cancer }\end{array}$ & $\begin{array}{l}\text { Bayesian pop-PK (w. } \\
\text { dexamethasone) }\end{array}$ & $\begin{array}{l}\mathrm{CL}[\mathrm{L} / \mathrm{h}]=31.5 \text {, Dexamethasone: } 6.05 \text { (additive), MissingDexamethasone: } \\
4.60 \text { (additive), } \\
\mathrm{V}_{1}[\mathrm{~L}]=13.9 \text {, Males: } 2.22 \text { (additive) }\end{array}$ & Covariates & $\begin{array}{l}\text { (Perez-Ruixo } \\
\text { et al., 2007) }\end{array}$ \\
\hline & $\begin{array}{l}\text { Advanced solid tumors, } \\
\text { breast cancer, melanoma, } \\
\text { soft tissue sarcoma, renal } \\
\text { cancer, colorectal cancer, } \\
\text { ovarian cancer }\end{array}$ & $\begin{array}{l}\text { Semi-mech. Pop- } \\
\text { PK/PD }\end{array}$ & & Dose, Toxicity, Covariates & $\begin{array}{l}\text { (Fetterly et al.., } \\
\underline{2008 \mathrm{~b})}\end{array}$ \\
\hline \multirow{4}{*}{ Trastuzumab } & $\begin{array}{l}\text { HER2-positive early breast } \\
\text { cancer }\end{array}$ & Pop-PK & $\begin{array}{l}\mathrm{CL}[\mathrm{L} / \text { day }]=0.111 \cdot(B W / 68)^{1.04} \cdot(\text { AlanineTransaminase } / 19)^{0.144} \\
\mathrm{Vc}[\mathrm{L}]=2.91 \cdot(B W / 68)^{0.443} \\
\mathrm{Vp}[\mathrm{L}]=3.06 \cdot(B W / 68)^{0.500}\end{array}$ & Covariates & $\begin{array}{l}\text { (Quartino et } \\
\text { al...2016) }\end{array}$ \\
\hline & $\begin{array}{l}\text { HER2-positive inoperable } \\
\text { cancer }\end{array}$ & Pop-PK & & DDIs & $\begin{array}{l}\text { (Eppler et al.. } \\
\underline{2015)}\end{array}$ \\
\hline & $\begin{array}{l}\text { HER2-positive breast } \\
\text { cancer }\end{array}$ & Pop-PK/PD & & Toxicity, Covariates & $\begin{array}{l}\text { (van Hasselt et } \\
\text { al., 2011) }\end{array}$ \\
\hline & $\begin{array}{l}\text { HER2-positive metastatic } \\
\text { breast cancer }\end{array}$ & Pop-PK & $\begin{array}{l}\mathrm{CL}[\mathrm{L} / \text { day }]=0.225 \cdot(\min (\mathrm{ECD}, 200) / 8.23) \cdot 0.041 \\
\mathrm{CL} \cdot(1+(0.221 \cdot \mathrm{MET})) \\
\mathrm{V}_{1}[\mathrm{~L}]=2.95 \cdot(\mathrm{BW} / 65) \cdot 0.556 \cdot(\min (\mathrm{EDC}, 200) / 8.23) \cdot 0.105\end{array}$ & Dose, Covariates & $\begin{array}{l}\text { (Bruno et al.. } \\
\underline{2005)}\end{array}$ \\
\hline \multirow[t]{4}{*}{ Trastuzumab emtansine } & $\begin{array}{l}\text { HER2-positive metastatic } \\
\text { breast cancer }\end{array}$ & $\begin{array}{l}\text { Semi-mech. Pop- } \\
\text { PK/PD }\end{array}$ & & Toxicity, Dose, Covariates & $\begin{array}{l}\text { (Bender et al.. } \\
\underline{2012)}\end{array}$ \\
\hline & $\begin{array}{l}\text { HER2-positive breast } \\
\text { cancer }\end{array}$ & Pop-PK & $\begin{array}{l}\mathrm{CL}[\mathrm{L} / \mathrm{day}]=\exp (0.676+0.49 \cdot(B W / 70)+0.035 \cdot \\
\log (\text { BaselineEpidermalGrowthFactor } / 25)-0.423 \cdot \log (\text { Albumin } / 41) \\
+0.052 \cdot \log (\text { BaselineSumLongestLesions } / 9)-0.002 \cdot \text { BaselineTrastuzumab } \\
+0.071 \cdot(\text { AspartateAminotransferase } / 25) \\
\mathrm{V}_{\mathrm{c}}[\mathrm{L}]=\exp (3.127+0.596 \cdot \log (\text { BW/70) })\end{array}$ & $\begin{array}{l}\text { Renal impairment, Ethnicity, } \\
\text { Covariates }\end{array}$ & $\begin{array}{l}\text { (Lu et al.. } \\
\underline{2014 b)}\end{array}$ \\
\hline & $\begin{array}{l}\text { HER2-positive metastatic } \\
\text { breast cancer }\end{array}$ & Semi-mech. Pop-PK & & Covariates & $\begin{array}{l}\text { (Chudasama et } \\
\text { al.. 2012) }\end{array}$ \\
\hline & HER2-positive locally & Pop-PK & & Ethnicity & $\begin{array}{l}(\text { Li et al.., } \\
\underline{\underline{2016})}\end{array}$ \\
\hline
\end{tabular}


Pharmacokinetic models for precision dosing in oncology - Supplementary appendix

\begin{tabular}{|c|c|c|c|c|c|}
\hline & $\begin{array}{l}\text { advanced/metastatic breast } \\
\text { cancer }\end{array}$ & & & & \\
\hline & $\begin{array}{l}\text { HER2-positive breast } \\
\text { cancer }\end{array}$ & Pop-PK/PD & & Efficacy, Dose & $\begin{array}{l}\text { (Singh and } \\
\text { Shah, 2017) }\end{array}$ \\
\hline & $\begin{array}{l}\text { HER2-positive metastatic } \\
\text { breast cancer }\end{array}$ & Pop-PK & & Limited sampling & $\begin{array}{l}(\text { Lu et al.. } \\
\underline{2013)}\end{array}$ \\
\hline \multirow{2}{*}{ Triptorelin } & $\begin{array}{l}\text { Healthy volunteers, } \\
\text { Prostate cancer }\end{array}$ & Pop-PK/PD & & Efficacy & $\begin{array}{l}\text { (Tornoe et al.. } \\
\underline{2007)}\end{array}$ \\
\hline & $\begin{array}{l}\text { Healthy volunteers, } \\
\text { Prostate cancer }\end{array}$ & $\begin{array}{l}\text { Semi-mech. Pop- } \\
\text { PK/PD }\end{array}$ & & Efficacy, Formulation & $\begin{array}{l}\text { (Romero et al.. } \\
\underline{2012)}\end{array}$ \\
\hline \multirow[t]{2}{*}{ Troxacitabine } & $\begin{array}{l}\text { Advanced solid tumors, } \\
\text { Advanced leukemia }\end{array}$ & Pop-PK & $\begin{array}{l}\text { Model based on } \mathrm{mg} / \mathrm{h} \text { dosing: } \\
\mathrm{CL}-C L_{C R}, B S A \text {, } \\
\mathrm{V}_{1}-B S A \\
\mathrm{~V}_{2}-B S A, \\
\mathrm{Q}_{3}-B S A \text {, } \\
\text { Model based on } \mathrm{mg} / \mathrm{h} / \mathrm{BSA}\left[\mathrm{m}^{2}\right] \text { dosing: } \\
\mathrm{CL}-C L_{C R}\end{array}$ & Covariates & $\begin{array}{l}\text { (Lee et al.. } \\
\underline{2006)}\end{array}$ \\
\hline & Solid tumors & $\begin{array}{l}\text { Semi-mech. Pop- } \\
\text { PK/PD }\end{array}$ & & Toxicity & $\begin{array}{l}(\text { Ng et al.. } \\
\text { 2011) }\end{array}$ \\
\hline Veliparib & $\begin{array}{l}\text { BRCA 1/2-mutated cancer, } \\
\text { PARP-sensitive tumors }\end{array}$ & Pop-PK/PD & $\begin{array}{l}\mathrm{CL}_{\mathrm{R}} / \mathrm{F}[\mathrm{L} / \mathrm{h}]=17.3 \cdot 0.7 \cdot\left(\text { CL }_{C R} / 95\right)^{0.903} \\
\mathrm{~V}_{\mathrm{c}} / \mathrm{F}[\mathrm{L}]=99.2 \cdot(\text { LeanBodyMass } / 48)^{1.21}\end{array}$ & $\begin{array}{l}\text { Efficacy, Metabolite, } \\
\text { Covariates }\end{array}$ & $\begin{array}{l}\text { (Niu et al.. } \\
\text { 2017) }\end{array}$ \\
\hline Venetoclax & $\begin{array}{l}\text { Chronic lymphocytic } \\
\text { leukemia and non- } \\
\text { Hodgkin's lymphoma }\end{array}$ & Pop-PK & $\begin{array}{l}\text { CL/F[L/day }]=413, \text { ModerateCYP3Ainhibitor: } 0.807, \\
\text { StrongCYP3Ainhibitor: } 0.158, \text { Rituximab: } 1.21, \\
\mathrm{~V}_{2} / \mathrm{F}[\mathrm{L}]=113 \text {, Female: } 0.683, \text { CLL/SLL/NHL: } 1.79, \\
\mathrm{~F} 1=1.0 \text { (fixed), Fasting: } 0.334, \text { Fed: } 1.24, \text { ModerateFat: } 1.26, \text { HighFat: } \\
1.42\end{array}$ & $\begin{array}{l}\text { Food effects, DDIs, Dose, } \\
\text { Covariates }\end{array}$ & $\begin{array}{l}\text { (Jones et al.. } \\
\underline{2016)}\end{array}$ \\
\hline \multirow[t]{4}{*}{ Vincristine } & Cancer (N/S) & Pop-PK & $\begin{array}{l}\mathrm{CL}-\mathrm{BW} \\
\mathrm{V}-\mathrm{BW}\end{array}$ & $\begin{array}{l}\text { Pediatrics, Metabolic } \\
\text { phenotype (CYP3A), Limited } \\
\text { sampling, Toxicity, Covariates }\end{array}$ & $\begin{array}{l}\text { (Moore et al.. } \\
\underline{2011)}\end{array}$ \\
\hline & $\begin{array}{l}\text { Acute lymphoblastic } \\
\text { leukemia }\end{array}$ & PK & & Pediatrics & $\begin{array}{l}\text { (de Graaf et } \\
\text { al., 1995) }\end{array}$ \\
\hline & $\begin{array}{l}\text { Acute lymphoblastic } \\
\text { leukemia, non-Hodgkin } \\
\text { lymphoma, Wilms' tumor }\end{array}$ & Bayesian pop-PK & & Pediatrics, Limited sampling & $\begin{array}{l}\text { (Gidding et al.. } \\
1999)\end{array}$ \\
\hline & $\begin{array}{l}\text { Lympholytic leukemia, } \\
\text { histocytic lymphoma, } \\
\text { multiple myeloma, } \\
\text { idiopathic } \\
\text { thrombocytopenic purpura, } \\
\text { lymphoma, Hodgkin's } \\
\text { disease }\end{array}$ & PK & & Investigational & $\begin{array}{l}\text { (Sethi et al.. } \\
\underline{1981)}\end{array}$ \\
\hline
\end{tabular}


Pharmacokinetic models for precision dosing in oncology - Supplementary appendix

\begin{tabular}{|c|c|c|c|c|c|}
\hline & Solid tumors & Bayesian pop-PK & & $\begin{array}{l}\text { Metabolic genotype } \\
(\text { CYP3A4/5), Transporter } \\
\text { genotype (ABCB1), Pediatrics, } \\
\text { Covariates }\end{array}$ & $\begin{array}{l}\text { (Guilhaumou } \\
\text { et al., 2011) }\end{array}$ \\
\hline \multirow{7}{*}{ Vinorelbine } & $\begin{array}{l}\text { Breast, prostate, lung, } \\
\text { bladder, other cancer }\end{array}$ & Pop-PK & & Toxicity, Dose, Covariates & $\begin{array}{l}\text { (Wong et al.. } \\
\underline{2006)}\end{array}$ \\
\hline & Metastatic breast cancer & Pop-PK & $\begin{array}{l}\mathrm{CL}[\mathrm{L} / \mathrm{h}]=74.2 \cdot\left(B_{S A} / A_{i} B_{\text {median }}\right)^{1.25} \text {. } \\
\left(\text { AlkalinePhosphate }_{i} / \text { AlkalinePhosphate }_{\text {median }}\right)^{-0.25}\end{array}$ & Dose, Covariates & $\begin{array}{l}\text { (Deporte-Fety } \\
\text { et al., 2004) }\end{array}$ \\
\hline & Non-small cell lung cancer & Pop-PK & & $\begin{array}{l}\text { Other special populations } \\
\text { (elderly) }\end{array}$ & $\begin{array}{l}\text { (Puozzo and } \\
\text { Gridelli, 2004) }\end{array}$ \\
\hline & $\begin{array}{l}\text { Advanced metastatic } \\
\text { cancer }\end{array}$ & Bayesian pop-PK & & $\begin{array}{l}\text { Other special populations } \\
\text { (elderly) }\end{array}$ & $\begin{array}{l}\text { (Gauvin et al.. } \\
\underline{2000)}\end{array}$ \\
\hline & Non-small cell lung cancer & Bayesian pop-PK & & Dose & $\begin{array}{l}\text { (Sabot et al.. } \\
\text { 1998) }\end{array}$ \\
\hline & $\begin{array}{l}\text { Advanced metastatic } \\
\text { cancer }\end{array}$ & Bayesian pop-PK & $\mathrm{CL}-($ Elderly $)$ & Limited sampling & $\begin{array}{l}\text { (Gauvin et al.. } \\
\text { 2002) }\end{array}$ \\
\hline & $\begin{array}{l}\text { HER2-positive locally } \\
\text { advanced or metastatic } \\
\text { breast cancer }\end{array}$ & Pop-PK (w. lapatinib) & $\begin{array}{l}\mathrm{CL}[\mathrm{L} / \mathrm{h}]=24.9 \cdot\left(\text { PlateletCount }_{i} / 250000\right)^{-1.1} \cdot\left(\text { BW/70 }^{0.75},\right. \\
\mathrm{V} 1, \mathrm{~V} 2, \mathrm{~V} 3-(\mathrm{BW} / 70) \\
\mathrm{Q} 2, \mathrm{Q} 3-(\mathrm{BW} / 70)^{0.75}\end{array}$ & Dose, Covariates & $\begin{array}{l}\text { (Rezai et al.. } \\
\text { 2011) }\end{array}$ \\
\hline Vinzolidine & $\begin{array}{l}\text { Breast cancer, melanoma, } \\
\text { Renal cancer }\end{array}$ & PK & & Investigational & $\begin{array}{l}\text { (Taylor et al.. } \\
\underline{1990)}\end{array}$ \\
\hline \multicolumn{6}{|c|}{$\begin{array}{l}\text { Footnotes: } \\
\text { AAG: } \alpha_{1} \text {-acid glycoprotein; BMI: body mass index; BSA: body surface area; BW: bodyweight; } \mathrm{CL}_{\mathrm{CR}} \text { : creatinine clearance; CNS: central nervous system; DDIs: drug-drug interactions; eGFR: estimated glomerular } \\
\text { filtration rate; GFR: glomerular filtration rate; IBW: ideal bodyweight; N/A: not applicable; N/S: not stated; PBPK: physiologically-based pharmacokinetics; PD: pharmacodynamic; PK: pharmacokinetic; pop: } \\
\text { population; } \mathrm{S}_{\mathrm{CR}} \text { : serum creatinine; w.: with. }\end{array}$} \\
\hline
\end{tabular}

\section{References}

Abdel-Rahman SM, Breitkreutz ML, Bi C, Matzuka BJ, Dalal J, Casey KL, Garg U, Winkle S, Leeder JS, Breedlove J, and Rivera B (2016a) Design and Testing of an EHR-Integrated, Busulfan Pharmacokinetic Decision Support Tool for the Point-of-Care Clinician. Front Pharmacol 7:65.

Abdel-Rahman SM, Casey KL, Garg U, and Dalal J (2016b) Intravenous busulfan dose individualization - impact of modeling approach on dose recommendation. Pediatric transplantation 20:443-448.

Ahamadi M, Freshwater T, Prohn M, Li CH, de Alwis DP, de Greef R, Elassaiss-Schaap J, Kondic A, and Stone JA (2017) Model-Based Characterization of the Pharmacokinetics of Pembrolizumab: A Humanized Anti-PD-1 Monoclonal Antibody in Advanced Solid Tumors. CPT: pharmacometrics \& systems pharmacology 6:49-57.

Ahn BJ, Choi MK, Park YS, Lee J, Park SH, Park JO, Lim HY, Kang WK, Ko JW, and Yim DS (2010) Population pharmacokinetics of CPT-11 (irinotecan) in gastric cancer patients with peritoneal seeding after its intraperitoneal administration. Eur J Clin Pharmacol 66:1235-1245. 
Pharmacokinetic models for precision dosing in oncology - Supplementary appendix

Ajani JA, Takimoto C, Becerra CR, Silva A, Baez L, Cohn A, Major P, Kamida M, Feit K, and De Jager R (2005) A phase II clinical and pharmacokinetic study of intravenous exatecan mesylate (DX-8951f) in patients with untreated metastatic gastric cancer. Investigational new drugs 23:479-484.

Albrecht CF, Kruger PB, Smit BJ, Freestone M, Gouws L, Miller R, and Vanjaarsveld PP (1995) The Pharmacokinetic Behavior of Hypoxoside Taken Orally by Patients with Lung-Cancer in a Phase-I Trial. South African Medical Journal 85:861-865.

Ames MM, Miser JS, Smithson WA, Coccia PF, Hughes CS, and Davis DM (1982) Pharmacokinetic Study of Indicine N-Oxide in Pediatric CancerPatients. Cancer Chemoth Pharm 10:43-46.

Aoyama Y, Kaibara A, Takada A, Nishimura T, Katashima M, and Sawamoto T (2013) Population pharmacokinetic modeling of sepantronium bromide (YM155), a small molecule survivin suppressant, in patients with non-small cell lung cancer, hormone refractory prostate cancer, or unresectable stage III or IV melanoma. Investigational new drugs 31:443-451.

Azzopardi N, Lecomte T, Ternant D, Boisdron-Celle M, Piller F, Morel A, Gouilleux-Gruart V, Vignault-Desvignes C, Watier H, Gamelin E, and Paintaud G (2011) Cetuximab pharmacokinetics influences progression-free survival of metastatic colorectal cancer patients. Clinical cancer research : an official journal of the American Association for Cancer Research 17:6329-6337.

Bajaj G, Wang X, Agrawal S, Gupta M, Roy A, and Feng Y (2017) Model-Based Population Pharmacokinetic Analysis of Nivolumab in Patients With Solid Tumors. Cpt-Pharmacomet Syst 6:58-66.

Barpe DR, Rosa DD, and Froehlich PE (2010) Pharmacokinetic evaluation of doxorubicin plasma levels in normal and overweight patients with breast cancer and simulation of dose adjustment by different indexes of body mass. European Journal of Pharmaceutical Sciences 41:458-463.

Barrett JS, Mondick JT, Narayan M, Vijayakumar K, and Vijayakumar S (2008) Integration of modeling and simulation into hospital-based decision support systems guiding pediatric pharmacotherapy. BMC medical informatics and decision making 8:6.

Bartelink IH, Boelens JJ, Bredius RG, Egberts AC, Wang C, Bierings MB, Shaw PJ, Nath CE, Hempel G, Zwaveling J, Danhof M, and Knibbe CA (2012a) Body weight-dependent pharmacokinetics of busulfan in paediatric haematopoietic stem cell transplantation patients: towards individualized dosing. Clin Pharmacokinet 51:331-345.

Bartelink IH, Lalmohamed A, van Reij EM, Dvorak CC, Savic RM, Zwaveling J, Bredius RG, Egberts AC, Bierings M, Kletzel M, Shaw PJ, Nath CE, Hempel G, Ansari M, Krajinovic M, Theoret Y, Duval M, Keizer RJ, Bittencourt H, Hassan M, Gungor T, Wynn RF, Veys P, Cuvelier GD, Marktel S, Chiesa R, Cowan MJ, Slatter MA, Stricherz MK, Jennissen C, Long-Boyle JR, and Boelens JJ (2016) Busulfan Exposure Predicts Event Free Survival and Toxicity after Hematopoietic Cell Transplantation in Children And Young Adults: A Multicenter Retrospective Cohort Analysis. Bone marrow transplantation 51:S14-S14.

Bartelink IH, van Kesteren C, Boelens JJ, Egberts TC, Bierings MB, Cuvelier GD, Wynn RF, Slatter MA, Chiesa R, Danhof M, and Knibbe CA (2012b) Predictive performance of a busulfan pharmacokinetic model in children and young adults. Ther Drug Monit 34:574-583.

Bastian G, Barrail A, and Urien S (2003) Population pharmacokinetics of oxaliplatin in patients with metastatic cancer. Anti-cancer drugs 14:817-824.

Bellnier DA, Greco WR, Loewen GM, Nava H, Oseroff AR, and Dougherty TJ (2006) Clinical pharmacokinetics of the PDT photosensitizers porfimer sodium (Photofrin), 2-[1-hexyloxyethyl]-2-devinyl pyropheophorbide-a (Photochlor) and 5-ALA-induced protoporphyrin IX. Laser Surg Med 38:439-444. 
Pharmacokinetic models for precision dosing in oncology - Supplementary appendix

Bellnier DA, Greco WR, Loewen GM, Nava H, Oseroff AR, Pandey RK, Tsuchida T, and Dougherty TJ (2003) Population pharmacokinetics of the photodynamic therapy agent 2-[1-hexyloxyethyl]-2-devinyl pyropheophorbide-a in cancer patients. Cancer Res 63:1806-1813.

Bender BC, Schaedeli-Stark F, Koch R, Joshi A, Chu YW, Rugo H, Krop IE, Girish S, Friberg LE, and Gupta M (2012) A population pharmacokinetic/pharmacodynamic model of thrombocytopenia characterizing the effect of trastuzumab emtansine (T-DM1) on platelet counts in patients with HER2-positive metastatic breast cancer. Cancer Chemother Pharmacol 70:591-601.

Berg AK, Buckner JC, Galanis E, Jaeckle KA, Ames MM, and Reid JM (2015) Quantification of the impact of enzyme-inducing antiepileptic drugs on irinotecan pharmacokinetics and SN-38 exposure. J Clin Pharmacol 55:1303-1312.

Berg AK, Mandrekar SJ, Ziegler KL, Carlson EC, Szabo E, Ames MM, Boring D, Limburg PJ, Reid JM, and Cancer Prevention N (2013) Population pharmacokinetic model for cancer chemoprevention with sulindac in healthy subjects. J Clin Pharmacol 53:403-412.

Bergmann TK, Brasch-Andersen C, Green H, Mirza M, Pedersen RS, Nielsen F, Skougaard K, Wihl J, Keldsen N, Damkier P, Friberg LE, Peterson C, Vach W, Karlsson MO, and Brosen K (2011) Impact of CYP2C8*3 on paclitaxel clearance: a population pharmacokinetic and pharmacogenomic study in 93 patients with ovarian cancer. Pharmacogenomics $J$ 11:113-120.

Bi Y, Deng J, Murry DJ, and An G (2016) A Whole-Body Physiologically Based Pharmacokinetic Model of Gefitinib in Mice and Scale-Up to Humans. AAPS J 18:228-238.

Blair EYL, Rivory LP, Clarke SJ, and McLachlan AJ (2004) Population pharmacokinetics of raltitrexed in patients with advanced solid tumours. $B r J$ Clin Pharmacol 57:416-426.

Bonate PL, Craig A, Gaynon P, Gandhi V, Jeha S, Kadota R, Lam GN, Plunkett W, Razzouk B, Rytting M, Steinherz P, and Weitman S (2004) Population pharmacokinetics of clofarabine, a second-generation nucleoside analog, in pediatric patients with acute leukemia. $J C l i n$ Pharmacol 44:1309-1322.

Bonetti A, Franceschi T, Apostoli P, Cetto GL, Recaldin E, Molino A, and Leone R (1994) Cisplatin Pharmacokinetics in Elderly Patients. Ther Drug Monit 16:477-482.

Boni JP, Leister C, Bender G, Fitzpatrick V, Twine N, Stover J, Dorner A, Immermann F, and Burczynski ME (2005) Population pharmacokinetics of CCI-779: correlations to safety and pharmacogenomic responses in patients with advanced renal cancer. Clinical pharmacology and therapeutics 77:76-89.

Booth BP, Rahman A, Dagher R, Griebel D, Lennon S, Fuller D, Sahajwalla C, Mehta M, and Gobburu JV (2007) Population pharmacokinetic-based dosing of intravenous busulfan in pediatric patients. J Clin Pharmacol 47:101-111.

Bosch TM, Huitema AD, Doodeman VD, Jansen R, Witteveen E, Smit WM, Jansen RL, van Herpen CM, Soesan M, Beijnen JH, and Schellens JH (2006) Pharmacogenetic screening of CYP3A and ABCB1 in relation to population pharmacokinetics of docetaxel. Clin Cancer Res 12:57865793.

Boucaud M, Pinguet F, Culine S, Poujol S, Astre C, Gomeni R, and Bressolle F (2003) Modeling plasma and saliva topotecan concentration time course using a population approach. Oncol Res 13:211-219.

Bressolle F, Joulia JM, Pinguet F, Ychou M, Astre C, Duffour J, and Gomeni R (1999) Circadian rhythm of 5-fluorouracil population pharmacokinetics in patients with metastatic colorectal cancer. Cancer Chemother Pharmacol 44:295-302. 
Pharmacokinetic models for precision dosing in oncology - Supplementary appendix

Brown K, Comisar C, Witjes H, Maringwa J, de Greef R, Vishwanathan K, Cantarini M, and Cox E (2017) Population pharmacokinetics and exposureresponse of osimertinib in patients with non-small cell lung cancer. Br J Clin Pharmacol 83:1216-1226.

Bruno R, Vivier N, Vergniol JC, De Phillips SL, Montay G, and Sheiner LB (1996) A population pharmacokinetic model for docetaxel (Taxotere): model building and validation. $J$ Pharmacokinet Biopharm 24:153-172.

Bruno R, Vivier N, Veyrat-Follet C, Montay G, and Rhodes GR (2001) Population pharmacokinetics and pharmacokinetic-pharmacodynamic relationships for docetaxel. Investigational new drugs 19:163-169.

Bruno R, Washington CB, Lu JF, Lieberman G, Banken L, and Klein P (2005) Population pharmacokinetics of trastuzumab in patients with HER2+ metastatic breast cancer. Cancer Chemother Pharmacol 56:361-369.

Bulitta JB, Zhao P, Arnold RD, Kessler DR, Daifuku R, Pratt J, Luciano G, Hanauske AR, Gelderblom H, Awada A, and Jusko WJ (2009) Mechanistic population pharmacokinetics of total and unbound paclitaxel for a new nanodroplet formulation versus Taxol in cancer patients. Cancer Chemother Pharmacol 63:1049-1063.

Caulet M, Lecomte T, Bouche O, Rollin J, Gouilleux-Gruart V, Azzopardi N, Leger J, Borg C, Douillard JY, Manfredi S, Smith D, Capitain O, Ferru A, Moussata D, Terrebone E, Paintaud G, and Ternant D (2016) Bevacizumab Pharmacokinetics Influence Overall and Progression-Free Survival in Metastatic Colorectal Cancer Patients. Clin Pharmacokinet 55:1381-1394.

Chalret du Rieu Q, Fouliard S, White-Koning M, Kloos I, Chatelut E, and Chenel M (2014a) Pharmacokinetic/Pharmacodynamic modeling of abexinostat-induced thrombocytopenia across different patient populations: application for the determination of the maximum tolerated doses in both lymphoma and solid tumour patients. Investigational new drugs 32:985-994.

Chalret du Rieu Q, White-Koning M, Picaud L, Lochon I, Marsili S, Gladieff L, Chatelut E, and Ferron G (2014b) Population pharmacokinetics of peritoneal, plasma ultrafiltrated and protein-bound oxaliplatin concentrations in patients with disseminated peritoneal cancer after intraperitoneal hyperthermic chemoperfusion of oxaliplatin following cytoreductive surgery: correlation between oxaliplatin exposure and thrombocytopenia. Cancer Chemother Pharmacol 74:571-582.

Chan KK, Cohen JL, Gross JF, Himmelstein KJ, Bateman JR, Tsulee Y, and Marlis AS (1978) Prediction of Adriamycin Disposition in Cancer-Patients Using a Physiologic, Pharmacokinetic Model. Cancer Treat Rep 62:1161-1171.

Chatelut E, Pivot X, Otto J, Chevreau C, Thyss A, Renee N, Milano G, and Canal P (2000) A limited sampling strategy for determining carboplatin AUC and monitoring drug dosage. Eur J Cancer 36:264-269.

Chen N, Brachmann C, Liu X, Pierce DW, Dey J, Kerwin WS, Li Y, Zhou S, Hou S, Carleton M, Klinghoffer RA, Palmisano M, and Chopra R (2015) Albumin-bound nanoparticle (nab) paclitaxel exhibits enhanced paclitaxel tissue distribution and tumor penetration. Cancer Chemother Pharmacol 76:699-712

Chen N, Li Y, Ye Y, Palmisano M, Chopra R, and Zhou S (2014) Pharmacokinetics and pharmacodynamics of nab-paclitaxel in patients with solid tumors: disposition kinetics and pharmacology distinct from solvent-based paclitaxel. J Clin Pharmacol 54:1097-1107.

Chen TL, Passos-Coelho JL, Noe DA, Kennedy MJ, Black KC, Colvin OM, and Grochow LB (1995) Nonlinear pharmacokinetics of cyclophosphamide in patients with metastatic breast cancer receiving high-dose chemotherapy followed by autologous bone marrow transplantation. Cancer research 55:810-816. 
Pharmacokinetic models for precision dosing in oncology - Supplementary appendix

Chiu YL, Carlson DM, Pradhan RS, and Ricker JL (2013) Exposure-response (safety) analysis to identify linifanib dose for a Phase III study in patients with hepatocellular carcinoma. Clin Ther 35:1770-1777.

Chudasama VL, Schaedeli Stark F, Harrold JM, Tibbitts J, Girish SR, Gupta M, Frey N, and Mager DE (2012) Semi-mechanistic population pharmacokinetic model of multivalent trastuzumab emtansine in patients with metastatic breast cancer. Clin Pharmacol Ther 92:520-527.

Claret L, Lu JF, Sun YN, and Bruno R (2010) Development of a modeling framework to simulate efficacy endpoints for motesanib in patients with thyroid cancer. Cancer Chemother Pharmacol 66:1141-1149.

Climente-Marti M, Merino-Sanjuan M, Almenar-Cubells D, and Jimenez-Torres NV (2003) A Bayesian method for predicting 5-fluorouracil pharmacokinetic parameters following short-term infusion in patients with colorectal cancer. J Pharm Sci 92:1155-1165.

Comets E, Ikeda K, Hoff P, Fumoleau P, Wanders J, and Tanigawara Y (2003) Comparison of the pharmacokinetics of S-1, an oral anticancer agent, in Western and Japanese patients. J Pharmacokinet Pharmacodyn 30:257-283.

Coustere C, Mentre F, Sommadossi JP, Diasio RB, and Steimer JL (1991) A mathematical model of the kinetics of 5-fluorouracil and its metabolites in cancer patients. Cancer Chemother Pharmacol 28:123-129.

Daher Abdi Z, Lavau-Denes S, Premaud A, Urien S, Sauvage FL, Martin J, Leobon S, Marquet P, Tubiana-Mathieu N, and Rousseau A (2014) Pharmacokinetics and exposure-effect relationships of capecitabine in elderly patients with breast or colorectal cancer. Cancer Chemother Pharmacol 73:1285-1293.

Darwish M, Megason G, Bond M, Hellriegel E, Robertson P, Jr., Grasela T, and Phillips L (2014) Population pharmacokinetics and pharmacokinetics/pharmacodynamics of bendamustine in pediatric patients with relapsed/refractory acute leukemia. Current medical research and opinion 30:2305-2315.

Davda JP, Jain M, Batra SK, Gwilt PR, and Robinson DH (2008) A physiologically based pharmacokinetic (PBPK) model to characterize and predict the disposition of monoclonal antibody CC49 and its single chain Fv constructs. International immunopharmacology 8:401-413.

De Buck SS, Jakab A, Boehm M, Bootle D, Juric D, Quadt C, and Goggin TK (2014) Population pharmacokinetics and pharmacodynamics of BYL719, a phosphoinositide 3-kinase antagonist, in adult patients with advanced solid malignancies. Br J Clin Pharmacol 78:543-555.

de Castro FA, Piana C, Simoes BP, Lanchote VL, and Della Pasqua O (2015) Busulfan dosing algorithm and sampling strategy in stem cell transplantation patients. Br J Clin Pharmacol 80:618-629.

de Graaf SS, Bloemhof H, Vendrig DE, and Uges DR (1995) Vincristine disposition in children with acute lymphoblastic leukemia. Medical and pediatric oncology 24:235-240.

de Jong FA, Mathijssen RHJ, Xie RJ, Verweij J, and Sparreboom A (2004) Flat-fixed dosing of irinotecan: Influence on pharmacokinetic and pharmacodynamic variability. Clinical cancer research : an official journal of the American Association for Cancer Research 10:4068-4071.

de Jongh FE, Gallo JM, Shen MY, Verweij J, and Sparreboom A (2004) Population pharmacokinetics of cisplatin in adult cancer patients. Cancer Chemother Pharmacol 54:105-112.

de Wit D, Schneider TC, Moes DJ, Roozen CF, den Hartigh J, Gelderblom H, Guchelaar HJ, van der Hoeven JJ, Links TP, Kapiteijn E, and van Erp NP (2016) Everolimus pharmacokinetics and its exposure-toxicity relationship in patients with thyroid cancer. Cancer Chemother Pharmacol 78:63-71. 
Pharmacokinetic models for precision dosing in oncology - Supplementary appendix

de Zwart L, Snoeys J, De Jong J, Sukbuntherng J, Mannaert E, and Monshouwer M (2016) Ibrutinib Dosing Strategies Based on Interaction Potential of CYP3A4 Perpetrators Using Physiologically Based Pharmacokinetic Modeling. Clin Pharmacol Ther 100:548-557.

Delbaldo C, Chatelut E, Re M, Deroussent A, Seronie-Vivien S, Jambu A, Berthaud P, Le Cesne A, Blay JY, and Vassal G (2006) Pharmacokineticpharmacodynamic relationships of imatinib and its main metabolite in patients with advanced gastrointestinal stromal tumors. Clin Cancer Res 12:6073-6078.

Delord JP, Umlil A, Guimbaud R, Gregoire N, Lafont T, Canal P, Bugat R, and Chatelut E (2003) Population pharmacokinetics of oxaliplatin. Cancer Chemother Pharmacol 51:127-131.

Deporte-Fety R, Simon N, Fumoleau P, Campone M, Kerbrat P, Bonneterre J, Fargeot P, and Urien S (2004) Population pharmacokinetics of short intravenous vinorelbine infusions in patients with metastatic breast cancer. Cancer Chemother Pharmacol 53:233-238.

Di Paolo A, Danesi R, Vannozzi F, Falcone A, Mini E, Cionini L, Ibrahim T, Amadori D, and Del Tacca M (2002) Limited sampling model for the analysis of 5-fluorouracil pharmacokinetics in adjuvant chemotherapy for colorectal cancer. Clinical pharmacology and therapeutics 72:627637.

Dickschen K, Willmann S, Thelen K, Lippert J, Hempel G, and Eissing T (2012) Physiologically Based Pharmacokinetic Modeling of Tamoxifen and its Metabolites in Women of Different CYP2D6 Phenotypes Provides New Insight into the Tamoxifen Mass Balance. Front Pharmacol 3:92.

Dickschen KJR, Willmann S, Hempel G, and Block M (2017) Addressing Adherence Using Genotype-Specific PBPK Modeling-Impact of Drug Holidays on Tamoxifen and Endoxifen Plasma Levels. Front Pharmacol 8.

Diestelhorst C, Boos J, McCune JS, Russell J, Kangarloo SB, and Hempel G (2014) Predictive performance of a physiologically based pharmacokinetic model of busulfan in children. Pediatric hematology and oncology 31:731-742.

Dombrowsky E, Jayaraman B, Narayan M, and Barrett JS (2011) Evaluating performance of a decision support system to improve methotrexate pharmacotherapy in children and young adults with cancer. Ther Drug Monit 33:99-107.

Duffull SB, Begg EJ, Robinson BA, and Deely JJ (1997) A sequential Bayesian algorithm for dose individualisation of carboplatin. Cancer Chemother Pharmacol 39:317-326.

Duong JK, Griffin MJ, Hargrave D, Vormoor J, Edwards D, and Boddy AV (2017) A population pharmacokinetic model of AT9283 in adults and children to predict the maximum tolerated dose in children with leukaemia. Br J Clin Pharmacol.

Dupuis C, Mercier C, Yang C, Monjanel-Mouterde S, Ciccolini J, Fanciullino R, Pourroy B, Deville JL, Duffaud F, Bagarry-Liegey D, Durand A, Iliadis A, and Favre R (2008) High-dose methotrexate in adults with osteosarcoma: a population pharmacokinetics study and validation of a new limited sampling strategy. Anti-cancer drugs 19:267-273.

Edwards AY, Elgart A, Farrell C, Barnett-Griness O, Rabinovich-Guilatt L, and Spiegelstein O (2017) A population pharmacokinetic meta-analysis of custirsen, an antisense oligonucleotide, in oncology patients and healthy subjects. Br J Clin Pharmacol.

Edwards AY, Skolnik JM, Dombrowsky E, Patel D, and Barrett JS (2012) Modeling and simulation approaches to evaluate pharmacokinetic sampling contamination from central venous catheters in pediatric pharmacokinetic studies of actinomycin-D: a report from the children's oncology group. Cancer Chemother Pharmacol 70:83-94. 
Pharmacokinetic models for precision dosing in oncology - Supplementary appendix

Einolf HJ, Zhou J, Won C, Wang L, and Rebello S (2017) A Physiologically-Based Pharmacokinetic Modeling Approach To Predict Drug-Drug Interactions of Sonidegib (LDE225) with Perpetrators of CYP3A in Cancer Patients. Drug metabolism and disposition: the biological fate of chemicals 45:361-374.

Ekhart C, de Jonge ME, Huitema ADR, Schellens JHM, Rodenhuis S, and Beijnen JH (2006) Flat dosing of carboplatin is justified in adult patients with normal renal function. Clinical cancer research : an official journal of the American Association for Cancer Research 12:6502-6508.

Emoto C, Fukuda T, Cox S, Christians U, and Vinks AA (2013) Development of a Physiologically-Based Pharmacokinetic Model for Sirolimus: Predicting Bioavailability Based on Intestinal CYP3A Content. CPT: pharmacometrics \& systems pharmacology 2:e59.

Eppler S, Gordon MS, Redfern CH, Trudeau C, Xu N, Han K, and Lum BL (2015) Lack of a pharmacokinetic interaction between trastuzumab and carboplatin in the presence of docetaxel: results from a phase Ib study in patients with HER2-positive metastatic or locally advanced inoperable solid tumors. Anti-cancer drugs 26:448-455.

Evans WE, Crom WR, Tsiatis A, Green AA, Hayes FA, and Pratt CB (1982a) Pharmacokinetic modeling of cisplatin disposition in children and adolescents with cancer. Cancer Chemother Pharmacol 10:22-26.

Evans WE, Sinkule JA, Crom WR, Dow L, Look AT, and Rivera G (1982b) Pharmacokinetics of Teniposide (VM26) and etoposide (VP16-213) in children with cancer. Cancer Chemother Pharmacol 7:147-150.

Fang L, Holford NH, Hinkle G, Cao X, Xiao JJ, Bloomston M, Gibbs S, Saif OH, Dalton JT, Chan KK, Schlom J, Martin EW, Jr., and Sun D (2007) Population pharmacokinetics of humanized monoclonal antibody HuCC49deltaCH2 and murine antibody CC49 in colorectal cancer patients. $J$ Clin Pharmacol 47:227-237.

Farrell C, Schweizer C, Wustner J, Weil S, Namiki M, Nakano T, Nakai K, and Phillips MD (2012) Population pharmacokinetics of farletuzumab, a humanized monoclonal antibody against folate receptor alpha, in epithelial ovarian cancer. Cancer Chemother Pharmacol 70:727-734.

Feng Y, Masson E, Dai D, Parker SM, Berman D, and Roy A (2014) Model-based clinical pharmacology profiling of ipilimumab in patients with advanced melanoma. Br J Clin Pharmacol 78:106-117.

Ferron GM, Dai Y, and Semiond D (2013) Population pharmacokinetics of cabazitaxel in patients with advanced solid tumors. Cancer Chemother Pharmacol 71:681-692.

Fetterly GJ, Aras U, Meholick PD, Takimoto C, Seetharam S, McIntosh T, de Bono JS, Sandhu SK, Tolcher A, Davis HM, Zhou H, and Puchalski TA (2013) Utilizing pharmacokinetics/pharmacodynamics modeling to simultaneously examine free CCL2, total CCL2 and carlumab (CNTO 888) concentration time data. J Clin Pharmacol 53:1020-1027.

Fetterly GJ, Grasela TH, Sherman JW, Dul JL, Grahn A, Lecomte D, Fiedler-Kelly J, Damjanov N, Fishman M, Kane MP, Rubin EH, and Tan AR (2008a) Pharmacokinetic/pharmacodynamic modeling and simulation of neutropenia during phase I development of liposome-entrapped paclitaxel. Clin Cancer Res 14:5856-5863.

Fetterly GJ, Owen JS, Stuyckens K, Passarell JA, Zannikos P, Soto-Matos A, Izquierdo MA, and Perez-Ruixo JJ (2008b) Semimechanistic pharmacokinetic/pharmacodynamic model for hepatoprotective effect of dexamethasone on transient transaminitis after trabectedin (ET-743) treatment. Cancer Chemother Pharmacol 62:135-147. 
Pharmacokinetic models for precision dosing in oncology - Supplementary appendix

Foo J, Chmielecki J, Pao W, and Michor F (2012) Effects of pharmacokinetic processes and varied dosing schedules on the dynamics of acquired resistance to erlotinib in EGFR-mutant lung cancer. Journal of thoracic oncology : official publication of the International Association for the Study of Lung Cancer 7:1583-1593.

Fracasso ME, Apostoli P, Benoni G, Bonetti A, Grisco C, and Leone R (1987) Kinetics of Platinum in Cancer-Patients Treated with Cisplatin at Different Doses. Drug Exp Clin Res 13:367-372.

Freiwald M, Schmid U, Fleury A, Wind S, Stopfer P, and Staab A (2014) Population pharmacokinetics of afatinib, an irreversible ErbB family blocker, in patients with various solid tumors. Cancer Chemother Pharmacol 73:759-770.

Frenay M, Milano G, Renee N, Pons D, Khater R, Francois E, Thyss A, and Namer M (1989) Pharmacokinetics of weekly low dose doxorubicin. European journal of cancer \& clinical oncology 25:191-195.

Fujita K, Masuo Y, Okumura H, Watanabe Y, Suzuki H, Sunakawa Y, Shimada K, Kawara K, Akiyama Y, Kitamura M, Kunishima M, Sasaki Y, and Kato Y (2016) Increased Plasma Concentrations of Unbound SN-38, the Active Metabolite of Irinotecan, in Cancer Patients with Severe Renal Failure. Pharm Res 33:269-282.

Fukae M, Shiraishi Y, Hirota T, Sasaki Y, Yamahashi M, Takayama K, Nakanishi Y, and Ieiri I (2016) Population pharmacokinetic-pharmacodynamic modeling and model-based prediction of docetaxel-induced neutropenia in Japanese patients with non-small cell lung cancer. Cancer Chemother Pharmacol 78:1013-1023.

Garcia-Garcia I, Hernandez-Gonzalez I, Diaz-Machado A, Gonzalez-Delgado CA, Perez-Rodriguez S, Garcia-Vega Y, Campos-Mojena R, TueroIglesias AD, Valenzuela-Silva CM, Cruz-Ramirez A, Martin-Trujillo A, Santana-Milian H, Lopez-Saura PA, Bello-Rivero I, and Group C-AS (2016) Pharmacokinetic and pharmacodynamic characterization of a novel formulation containing co-formulated interferons alpha- $2 \mathrm{~b}$ and gamma in healthy male volunteers. BMC pharmacology \& toxicology 17:58.

Gardner SN (2002) Modeling multi-drug chemotherapy: tailoring treatment to individuals. Journal of theoretical biology 214:181-207.

Gauvin A, Pinguet F, Culine S, Astre C, Gomeni R, and Bressolle F (2000) Bayesian estimate of vinorelbine pharmacokinetic parameters in elderly patients with advanced metastatic cancer. Clinical cancer research : an official journal of the American Association for Cancer Research 6:2690-2695.

Gauvin A, Pinguet F, Culine S, Astre C, Gomeni R, and Bressolle F (2002) A limited-sampling strategy to estimate individual pharmacokinetic parameters of vinorelbine in elderly patients with advanced metastatic cancer. Anti-cancer drugs 13:473-480.

Gianni L, Kearns CM, Giani A, Capri G, Vigano L, Locatelli A, Bonadonna G, and Egorin MJ (1995) Nonlinear Pharmacokinetics and Metabolism of Paclitaxel and Its Pharmacokinetic/Pharmacodynamic Relationships in Humans. Journal of clinical oncology : official journal of the American Society of Clinical Oncology 13:180-190.

Gibiansky L, Passey C, Roy A, Bello A, and Gupta M (2016) Model-based pharmacokinetic analysis of elotuzumab in patients with relapsed/refractory multiple myeloma. $J$ Pharmacokinet Pharmacodyn 43:243-257.

Gibiansky L, Sutjandra L, Doshi S, Zheng J, Sohn W, Peterson MC, Jang GR, Chow AT, and Perez-Ruixo JJ (2012) Population pharmacokinetic analysis of denosumab in patients with bone metastases from solid tumours. Clin Pharmacokinet 51:247-260. 
Pharmacokinetic models for precision dosing in oncology - Supplementary appendix

Gidding CE, Meeuwsen-de Boer GJ, Koopmans P, Uges DR, Kamps WA, and de Graaf SS (1999) Vincristine pharmacokinetics after repetitive dosing in children. Cancer Chemother Pharmacol 44:203-209.

Gieschke R, Burger HU, Reigner B, Blesch KS, and Steimer JL (2003) Population pharmacokinetics and concentration-effect relationships of capecitabine metabolites in colorectal cancer patients. Br J Clin Pharmacol 55:252-263.

Gieschke R, Reigner B, Blesch KS, and Steimer JL (2002) Population pharmacokinetic analysis of the major metabolites of capecitabine. $J$ Pharmacokinet Pharmacodyn 29:25-47.

Glenn KJ, Yu LJ, Reddy MB, Fretland AJ, Parrott N, Hussain S, Palacios M, Vazvaei F, Zhi J, and Tuerck D (2016) Investigating the effect of autoinduction in cynomolgus monkeys of a novel anticancer MDM2 antagonist, idasanutlin, and relevance to humans. Xenobiotica 46:667-676.

Gonzalez-Sales M, Valenzuela B, Perez-Ruixo C, Fernandez Teruel C, Miguel-Lillo B, Soto-Matos A, and Perez-Ruixo JJ (2012) Population pharmacokinetic-pharmacodynamic analysis of neutropenia in cancer patients receiving PM00104 (Zalypsis((R))). Clin Pharmacokinet 51:751764.

Gosselin NH, Mouksassi MS, Lu JF, and Hsu CP (2015) Population pharmacokinetic modeling of motesanib and its active metabolite, M4, in cancer patients. Clinical pharmacology in drug development 4:463-472.

Gotta V, Widmer N, Montemurro M, Leyvraz S, Haouala A, Decosterd LA, Csajka C, and Buclin T (2012) Therapeutic drug monitoring of imatinib: Bayesian and alternative methods to predict trough levels. Clin Pharmacokinet 51:187-201.

Griffin TW, Bokhari F, Collins J, Stochl M, Bernier M, Gionet M, Siebecker D, Wertheimer M, Giroves ES, Greenfield L, Houston LL, Doherty PW, and Wilson J (1989) A Preliminary Pharmacokinetic Study of in-111-Labeled 260f9 Anti-(Breast Cancer) Antibody in Patients. Cancer Immunol Immun 29:43-50.

Griffiths H, Shelley MD, and Fish RG (1987) A modified pharmacokinetic model for platinum disposition in ovarian cancer patients receiving cisplatin. Eur J Clin Pharmacol 33:67-72.

Guilhaumou R, Simon N, Quaranta S, Verschuur A, Lacarelle B, Andre N, and Solas C (2011) Population pharmacokinetics and pharmacogenetics of vincristine in paediatric patients treated for solid tumour diseases. Cancer Chemother Pharmacol 68:1191-1198.

Guillet P, Monjanel S, Nicoara A, Duffaud F, Lacarelle B, BagarryLiegey D, Durand A, Catalin J, and Favre R (1997) A Bayesian dosing method for carboplatin given by continuous infusion for $120 \mathrm{~h}$. Cancer Chemother Pharmacol 40:143-149.

Gupta A, Hussein Z, Hassan R, Wustner J, Maltzman JD, and Wallin BA (2016a) Population pharmacokinetics and exposure-response relationship of amatuximab, an anti-mesothelin monoclonal antibody, in patients with malignant pleural mesothelioma and its application in dose selection. Cancer Chemother Pharmacol 77:733-743.

Gupta A, Jarzab B, Capdevila J, Shumaker R, and Hussein Z (2016b) Population pharmacokinetic analysis of lenvatinib in healthy subjects and patients with cancer. Br J Clin Pharmacol 81:1124-1133.

Gupta N, Huh Y, Hutmacher MM, Ottinger S, Hui AM, and Venkatakrishnan K (2015) Integrated nonclinical and clinical risk assessment of the investigational proteasome inhibitor ixazomib on the QTc interval in cancer patients. Cancer Chemother Pharmacol 76:507-516.

Han K, Jin J, Maia M, Lowe J, Sersch MA, and Allison DE (2014) Lower exposure and faster clearance of bevacizumab in gastric cancer and the impact of patient variables: analysis of individual data from AVAGAST phase III trial. AAPS J 16:1056-1063. 
Pharmacokinetic models for precision dosing in oncology - Supplementary appendix

Han K, Jin JY, Marchand M, Eppler S, Choong N, Hack SP, Tikoo N, Bruno R, Dresser M, Musib L, and Budha NR (2015) Population pharmacokinetics and dosing implications for cobimetinib in patients with solid tumors. Cancer Chemother Pharmacol 76:917-924.

Han K, Peyret T, Marchand M, Quartino A, Gosselin NH, Girish S, Allison DE, and Jin J (2016a) Population pharmacokinetics of bevacizumab in cancer patients with external validation. Cancer Chemother Pharmacol 78:341-351.

Han K, Peyret T, Quartino A, Gosselin NH, Gururangan S, Casanova M, Merks JH, Massimino M, Grill J, Daw NC, Navid F, Jin J, and Allison DE (2016b) Bevacizumab dosing strategy in paediatric cancer patients based on population pharmacokinetic analysis with external validation. $\mathrm{Br} J$ Clin Pharmacol 81:148-160.

Hanada K, Nishijima K, Ogata H, Atagi S, and Kawahara M (2001) Population pharmacokinetic analysis of cisplatin and its metabolites in cancer patients: possible misinterpretation of covariates for pharmacokinetic parameters calculated from the concentrations of unchanged cisplatin, ultrafiltered platinum and total platinum. Jpn J Clin Oncol 31:179-184.

Hanley MJ, Gupta N, Suryanarayan K, Neuwirth R, Esseltine D, Horton T, Aplenc R, Alonzo TA, Lu X, Milton A, Mould DR, and Venkatakrishnan K (2016) Population Pharmacokinetic (Pk) Analysis of Bortezomib (Btz) in Pediatric Leukemia Patients (Pts): Support for Body Surface Area (Bsa)-Based Dosing in Pts Aged 2-16 Yrs. Clinical pharmacology and therapeutics 99:S46-S46.

Harris PA and Gross JF (1975) Preliminary Pharmacokinetic Model for Adriamycin (Nsc-123127). Cancer Chemoth Rep 1 59:819-825.

Hassan M, Fasth A, Gerritsen B, Haraldsson A, Syruckova Z, van den Berg H, Sandstrom M, Karlsson M, Kumlien S, and Vossen J (1996) Busulphan kinetics and limited sampling model in children with leukemia and inherited disorders. Bone marrow transplantation 18:843-850.

Hassan M, Svensson US, Ljungman P, Bjorkstrand B, Olsson H, Bielenstein M, Abdel-Rehim M, Nilsson C, Johansson M, and Karlsson MO (1999) A mechanism-based pharmacokinetic-enzyme model for cyclophosphamide autoinduction in breast cancer patients. Br J Clin Pharmacol 48:669677.

Hawwa AF, Collier PS, Millership JS, McCarthy A, Dempsey S, Cairns C, and McElnay JC (2008) Population pharmacokinetic and pharmacogenetic analysis of 6-mercaptopurine in paediatric patients with acute lymphoblastic leukaemia. Br J Clin Pharmacol 66:826-837.

Hempel G, Relling MV, de Rossi G, Stary J, De Lorenzo P, Valsecchi MG, Barisone E, Boos J, and Pieters R (2010) Pharmacokinetics of daunorubicin and daunorubicinol in infants with leukemia treated in the interfant 99 protocol. Pediatric blood \& cancer 54:355-360.

Henningsson A, Karlsson MO, Vigano L, Gianni L, Verweij J, and Sparreboom A (2001) Mechanism-based pharmacokinetic model for paclitaxel. Journal of clinical oncology : official journal of the American Society of Clinical Oncology 19:4065-4073.

Henningsson A, Marsh S, Loos WJ, Karlsson MO, Garsa A, Mross K, Mielke S, Vigano L, Locatelli A, Verweij J, Sparreboom A, and McLeod HL (2005) Association of CYP2C8, CYP3A4, CYP3A5, and ABCB1 polymorphisms with the pharmacokinetics of paclitaxel. Clinical cancer research : an official journal of the American Association for Cancer Research 11:8097-8104.

Henningsson A, Sparreboom A, Sandstrom M, Freijs A, Larsson R, Bergh J, Nygren P, and Karlsson MO (2003) Population pharmacokinetic modelling of unbound and total plasma concentrations of paclitaxel in cancer patients. Eur J Cancer 39:1105-1114.

Henriksson P, Carlstrom K, Pousette A, Gunnarsson PO, Johansson CJ, Eriksson B, Altersgard-Brorsson AK, Nordle O, and Stege R (1999) Time for revival of estrogens in the treatment of advanced prostatic carcinoma? Pharmacokinetics, and endocrine and clinical effects, of a parenteral estrogen regimen. Prostate 40:76-82. 
Pharmacokinetic models for precision dosing in oncology - Supplementary appendix

Hing J, Perea-Ruixo J, Stuyckens K, Soto-Matos A, Lopez-Lazaro L, and Zannikos P (2008) Mechanism-based pharmacokinetic/pharmacodynamic meta-analysis of trabectedin (ET-743, Yondelis) induced neutropenia. Clinical pharmacology and therapeutics 83:130-143.

Hirose T, Fujita K, Nishimura K, Ishida H, Yamashita K, Sunakawa Y, Mizuno K, Miwa K, Nagashima F, Tanigawara Y, Adachi M, and Sasaki Y (2010) Pharmacokinetics of S-1 and CYP2A6 genotype in Japanese patients with advanced cancer. Oncol Rep 24:529-536.

Hong Y, Passos VQ, Huang PH, and Lau YY (2017) Population Pharmacokinetics of Ceritinib in Adult Patients With Tumors Characterized by Genetic Abnormalities in Anaplastic Lymphoma Kinase. J Clin Pharmacol 57:652-662.

Hooker AC, Ten Tije AJ, Carducci MA, Weber J, Garrett-Mayer E, Gelderblom H, Mcguire WP, Verweij J, Karlsson MO, and Baker SD (2008) Population pharmacokinetic model for docetaxel in patients with varying degrees of liver function: Incorporating cytochrome P450 3A activity measurements. Clinical pharmacology and therapeutics 84:111-118.

Horton TM, Blaney SM, Langevin AM, Kuhn J, Kamen B, Berg SL, Bernstein M, and Weitman S (2005) Phase I trial and pharmacokinetic study of raltitrexed in children with recurrent or refractory leukemia: a pediatric oncology group study. Clinical cancer research : an official journal of the American Association for Cancer Research 11:1884-1889.

Houk BE, Bello CL, Kang DW, and Amantea M (2009) A Population Pharmacokinetic Meta-analysis of Sunitinib Malate (SU11248) and Its Primary Metabolite (SU12662) in Healthy Volunteers and Oncology Patients. Clinical cancer research : an official journal of the American Association for Cancer Research 15:2497-2506.

Houk BE, Bello CL, Poland B, Rosen LS, Demetri GD, and Motzer RJ (2010) Relationship between exposure to sunitinib and efficacy and tolerability endpoints in patients with cancer: results of a pharmacokinetic/pharmacodynamic meta-analysis. Cancer Chemother Pharmacol 66:357-371.

$\mathrm{Hu}$ ZY, Lu J, and Zhao Y (2014) A physiologically based pharmacokinetic model of alvespimycin in mice and extrapolation to rats and humans. $\mathrm{Br} J$ Pharmacol 171:2778-2789.

Hudachek SF, Eckhardt SG, Hicks B, and Gustafson DL (2010) Population pharmacokinetic model of PI-88, a heparanase inhibitor. Cancer Chemother Pharmacol 65:743-753.

Hudachek SF and Gustafson DL (2013) Physiologically based pharmacokinetic model of lapatinib developed in mice and scaled to humans. $J$ Pharmacokinet Pharmacodyn 40:157-176.

Huitema AD, Mathot RA, Tibben MM, Rodenhuis S, and Beijnen JH (2001) A mechanism-based pharmacokinetic model for the cytochrome P450 drug-drug interaction between cyclophosphamide and thioTEPA and the autoinduction of cyclophosphamide. J Pharmacokinet Pharmacodyn 28:211-230

Jakobsen P, Steiness E, Bastholt L, Dalmark M, Lorenzen A, Petersen D, Gjedde SB, Sandberg E, Rose C, Nielsen OS, Mouridsen HT, and Jakobsen A (1991) Multiple-Dose Pharmacokinetics of Epirubicin at 4 Different Dose Levels - Studies in Patients with Metastatic Breast-Cancer. Cancer Chemoth Pharm 28:63-68.

Jen JF, Cutler DL, Pai SM, Batra VK, Affrime MB, Zambas DN, Heft S, and Hajian G (2000) Population pharmacokinetics of temozolomide in cancer patients. Pharm Res 17:1284-1289.

Jiang XM, Galettis P, Links M, Mitchell PL, and McLachlan AJ (2008) Population pharmacokinetics of gemcitabine and its metabolite in patients with cancer: effect of oxaliplatin and infusion rate. Br J Clin Pharmacol 65:326-333. 
Pharmacokinetic models for precision dosing in oncology - Supplementary appendix

Jin F, Gao Y, Zhou H, Fang L, Li X, and Ramanathan S (2016) Population pharmacokinetic modeling of idelalisib, a novel PI3Kdelta inhibitor, in healthy subjects and patients with hematologic malignancies. Cancer Chemother Pharmacol 77:89-98.

Jing J, Nelson C, Paik J, Shirasaka Y, Amory JK, and Isoherranen N (2017) Physiologically Based Pharmacokinetic Model of All-trans-Retinoic Acid with Application to Cancer Populations and Drug Interactions. J Pharmacol Exp Ther 361:246-258.

Jodrell DI, Reyno LM, Sridhara R, Eisenberger MA, Tkaczuk KH, Zuhowski EG, Sinibaldi VJ, Novak MJ, and Egorin MJ (1994) Suramin Development of a Population Pharmacokinetic Model and Its Use with Intermittent Short Infusions to Control Plasma Drug Concentration in Patients with Prostate-Cancer. Journal of clinical oncology : official journal of the American Society of Clinical Oncology 12:166-175.

Joerger M (2012) Covariate pharmacokinetic model building in oncology and its potential clinical relevance. AAPS J 14:119-132.

Joerger M, Burgers JA, Baas P, Doodeman VD, Smits PH, Jansen RS, Vainchtein LD, Rosing H, Huitema AD, Beijnen JH, and Schellens JH (2012) Gene polymorphisms, pharmacokinetics, and hematological toxicity in advanced non-small-cell lung cancer patients receiving cisplatin/gemcitabine. Cancer Chemother Pharmacol 69:25-33.

Joerger M, Huitema AD, van den Bongard HJ, Baas P, Schornagel JH, Schellens JH, and Beijnen JH (2006a) Determinants of the elimination of methotrexate and 7-hydroxy-methotrexate following high-dose infusional therapy to cancer patients. Br J Clin Pharmacol 62:71-80.

Joerger M, Huitema ADR, Huizing MT, Willemse PHB, de Graeff A, Rosing H, Schellens JHM, Beijnen JH, and Vermorken JB (2007a) Safety and pharmacology of paclitaxel in patients with impaired liver function: a population pharmacokinetic-pharmacodynamic study. $B r J C l i n$ Pharmacol 64:622-633.

Joerger M, Huitema ADR, Richel DJ, Dittrich C, Pavlidis N, Briasoulis E, Vermorken JB, Strocchi E, Martoni A, Sorio R, Sleeboom HP, Izquierdo MA, Jodrell DI, Calvert H, Boddy AV, Hollema H, Fety R, Van der Vijgh WJF, Hempel G, Chatelut E, Karlsson M, Wilkins J, Tranchand B, Schrijvers AHGJ, Twelves C, Beijnen JH, and Schellens JHM (2007b) Population pharmacokinetics and pharmacodynamics of paclitaxel and carboplatin in ovarian cancer patients: A study by the European Organization for Research and Treatment of Cancer-Pharmacology and Molecular Mechanisms Group and New Drug Development Group. Clinical cancer research : an official journal of the American Association for Cancer Research 13:6410-6418.

Joerger M, Huitema ADR, Richel DJ, Dittrich C, Pavlidis N, Briasoulis E, Vermorken JB, Strocchi E, Martoni A, Sorio R, Sleeboom HP, Izquierdo MA, Jodrell DI, Fety R, de Bruijn E, Hempel G, Karlsson M, Tranchand B, Schrijvers AHGJ, Twelves C, Beijnen JH, and Schellens JHM (2007c) Population pharmacokinetics and pharmacodynamics of doxorubicin and cyclophosphamide in breast cancer patients: A study by the EORTCPAMM-NDDG. Clin Pharmacokinet 46:1051-1068.

Joerger M, Huitema ADR, van den Bongard DHJG, Schellens JHM, and Beijnen JH (2006b) Quantitative effect of gender, age, liver function, and body size on the population pharmacokinetics of paclitaxel in patients with solid tumors. Clinical cancer research : an official journal of the American Association for Cancer Research 12:2150-2157.

Johansson AM, Hill N, Perisoglou M, Whelan J, Karlsson MO, and Standing JF (2011) A population pharmacokinetic/pharmacodynamic model of methotrexate and mucositis scores in osteosarcoma. Ther Drug Monit 33:711-718.

Jones AK, Freise KJ, Agarwal SK, Humerickhouse RA, Wong SL, and Salem AH (2016) Clinical Predictors of Venetoclax Pharmacokinetics in Chronic Lymphocytic Leukemia and Non-Hodgkin's Lymphoma Patients: a Pooled Population Pharmacokinetic Analysis. AAPS J 18:1192-1202. 
Pharmacokinetic models for precision dosing in oncology - Supplementary appendix

Kaefer A, Yang J, Noertersheuser P, Mensing S, Humerickhouse R, Awni W, and Xiong H (2014) Mechanism-based pharmacokinetic/pharmacodynamic meta-analysis of navitoclax (ABT-263) induced thrombocytopenia. Cancer Chemother Pharmacol 74:593-602.

Karlsson MO, Molnar V, Bergh J, Freijs A, and Larsson R (1998) A general model for time-dissociated pharmacokinetic-pharmacodynamic relationship exemplified by paclitaxel myelosuppression. Clinical pharmacology and therapeutics 63:11-25.

Karlsson MO, Molnar V, Freijs A, Nygren P, Bergh J, and Larsson R (1999) Pharmacokinetic models for the saturable distribution of paclitaxel. Drug metabolism and disposition: the biological fate of chemicals 27:1220-1223.

Keizer RJ, Gupta A, Mac Gillavry MR, Jansen M, Wanders J, Beijnen JH, Schellens JHM, Karlsson MO, and Huitema ADR (2010) A model of hypertension and proteinuria in cancer patients treated with the anti-angiogenic drug E7080. J Pharmacokinet Pharmacodyn 37:347-363.

Keizer RJ, Zamacona MK, Jansen M, Critchley D, Wanders J, Beijnen JH, Schellens JHM, and Huitema ADR (2009) Application of population pharmacokinetic modeling in early clinical development of the anticancer agent E7820. Investigational new drugs 27:140-152.

Keizer RJ, Zandvliet AS, Beijnen JH, Schellens JH, and Huitema AD (2012) Two-stage model-based design of cancer phase I dose escalation trials: evaluation using the phase I program of barasertib (AZD1152). Investigational new drugs 30:1519-1530.

Kerbusch T, vanPutten JW, Groen HJ, Huitema AD, Mathjt RA, and Beijnen JH (2001) Population pharmacokinetics of ifosfamide and its 2- and 3dechloroethylated and 4-hydroxylated metabolites in resistant small-cell lung cancer patients. Cancer Chemother Pharmacol 48:53-61.

Kersting G, Willmann S, Wurthwein G, Lippert J, Boos J, and Hempel G (2012) Physiologically based pharmacokinetic modelling of high- and lowdose etoposide: from adults to children. Cancer Chemother Pharmacol 69:397-405.

Kho Y, Jansman FG, Prins NH, Neef C, and Brouwers JR (2006) Population pharmacokinetics of oxaliplatin (85 mg/m2) in combination with 5fluorouracil in patients with advanced colorectal cancer. Therapeutic drug monitoring 28:206-211.

Kitchen BJ, Balis FM, Poplack DG, O'Brien M, Craig CE, and Adamson PC (1997) A pediatric phase I trial and pharmacokinetic study of thioguanine administered by continuous i.v. infusion. Clin Cancer Res 3:713-717.

Klein CE, Kastrissios H, Miller AA, Hollis D, Yu D, Rosner GL, Grinblatt DL, Larson RA, and Ratain MJ (2006) Pharmacokinetics, pharmacodynamics and adherence to oral topotecan in myelodysplastic syndromes: a Cancer and Leukemia Group B study. Cancer Chemother Pharmacol 57:199-206.

Kletting P, Kull T, Bunjes D, Mahren B, Luster M, Reske SN, and Glatting G (2010) Radioimmunotherapy with anti-CD66 antibody: improving the biodistribution using a physiologically based pharmacokinetic model. Journal of nuclear medicine : official publication, Society of Nuclear Medicine 51:484-491.

Kletting P, Maass C, Reske S, Beer AJ, and Glatting G (2015) Physiologically Based Pharmacokinetic Modeling Is Essential in 90Y-Labeled Anti-CD66 Radioimmunotherapy. PloS one 10:e127934.

Kletting P, Muller B, Erentok B, Schmaljohann J, Behrendt FF, Reske SN, Mottaghy FM, and Glatting G (2012) Differences in predicted and actually absorbed doses in peptide receptor radionuclide therapy. Medical physics 39:5708-5717.

Kloft C, Graefe EU, Tanswell P, Scott AM, Hofheinz R, Amelsberg A, and Karlsson MO (2004) Population pharmacokinetics of sibrotuzumab, a novel therapeutic monoclonal antibody, in cancer patients. Investigational new drugs 22:39-52. 
Pharmacokinetic models for precision dosing in oncology - Supplementary appendix

Kloft C, Siegert W, and Jaehde U (2003) Individualised dosing strategy for high-dose carboplatin in patients with germ cell cancer. Br J Cancer 89:787794.

Kloft C, Wallin J, Henningsson A, Chatelut E, and Karlsson MO (2006) Population pharmacokinetic-pharmacodynamic model for neutropenia with patient subgroup identification: comparison across anticancer drugs. Clinical cancer research : an official journal of the American Association for Cancer Research 12:5481-5490.

Koizumi K, DeNardo GL, DeNardo SJ, Hays MT, Hines HH, Scheibe PO, Peng JS, Macey DJ, Tonami N, and Hisada K (1986) Multicompartmental analysis of the kinetics of radioiodinated monoclonal antibody in patients with cancer. Journal of nuclear medicine : official publication, Society of Nuclear Medicine 27:1243-1254.

Kolesar J, Brundage RC, Pomplun M, Alberti D, Holen K, Traynor A, Ivy P, and Wilding G (2011) Population pharmacokinetics of 3-aminopyridine-2carboxaldehyde thiosemicarbazone (Triapine (R)) in cancer patients. Cancer Chemother Pharmacol 67:393-400.

Koolen SL, Oostendorp RL, Beijnen JH, Schellens JH, and Huitema AD (2010) Population pharmacokinetics of intravenously and orally administered docetaxel with or without co-administration of ritonavir in patients with advanced cancer. Br J Clin Pharmacol 69:465-474.

Krogh-Madsen M, Bender B, Jensen MK, Nielsen OJ, Friberg LE, and Honore PH (2012) Population pharmacokinetics of cytarabine, etoposide, and daunorubicin in the treatment for acute myeloid leukemia. Cancer Chemother Pharmacol 69:1155-1163.

Kuester K, Kovar A, Lupfert C, Brockhaus B, and Kloft C (2008) Population pharmacokinetic data analysis of three phase I studies of matuzumab, a humanised anti-EGFR monoclonal antibody in clinical cancer development. Br J Cancer 98:900-906.

Kuester K, Kovar A, Lupfert C, Brockhaus B, and Kloft C (2009) Refinement of the population pharmacokinetic model for the monoclonal antibody matuzumab: external model evaluation and simulations. Clin Pharmacokinet 48:477-487.

Kunarajah K, Hennig S, Norris RLG, Lobb M, Charles BG, Pinkerton R, and Moore AS (2017) Population pharmacokinetic modelling of doxorubicin and doxorubicinol in children with cancer: is there a relationship with cardiac troponin profiles? Cancer Chemother Pharmacol.

Lack NA, Green B, Dale DC, Calandra GB, Lee H, MacFarland RT, Badel K, Liles WC, and Bridger G (2005) A pharmacokinetic-pharmacodynamic model for the mobilization of CD34+ hematopoietic progenitor cells by AMD3100. Clinical pharmacology and therapeutics 77:427-436.

Latz JE, Chaudhary A, Ghosh A, and Johnson RD (2006a) Population pharmacokinetic analysis of ten phase II clinical trials of pemetrexed in cancer patients. Cancer Chemother Pharmacol 57:401-411.

Latz JE, Karlsson MO, Rusthoven JJ, Ghosh A, and Johnson RD (2006b) A semimechanistic-physiologic population pharmacokinetic/pharmacodynamic model for neutropenia following pemetrexed therapy. Cancer Chemother Pharmacol 57:412-426.

Launay-Iliadis MC, Bruno R, Cosson V, Vergniol JC, Oulid-Aissa D, Marty M, Clavel M, Aapro M, Le Bail N, and Iliadis A (1995) Population pharmacokinetics of docetaxel during phase I studies using nonlinear mixed-effect modeling and nonparametric maximum-likelihood estimation. Cancer Chemother Pharmacol 37:47-54.

Lee CK, Rowinsky EK, Li J, Giles F, Moore MJ, Hidalgo M, Capparelli E, Jolivet J, and Baker SD (2006) Population pharmacokinetics of troxacitabine, a novel dioxolane nucleoside analogue. Clinical cancer research : an official journal of the American Association for Cancer Research 12:2158-2165. 
Pharmacokinetic models for precision dosing in oncology - Supplementary appendix

Leijen S, Veltkamp SA, Huitema AD, van Werkhoven E, Beijnen JH, and Schellens JH (2013) Phase I dose-escalation study and population pharmacokinetic analysis of fixed dose rate gemcitabine plus carboplatin as second-line therapy in patients with ovarian cancer. Gynecologic oncology 130:511-517.

Leone R, Benoni G, Apostoli P, Bonetti A, Griso C, and Fracasso ME (1987) Two-cycle cisplatin kinetics in patients with ovarian and mammary cancer. Ther Drug Monit 9:374-377.

Lewis LD, Miller AA, Rosner GL, Dowell JE, Valdivieso M, Relling MV, Egorin MJ, Bies RR, Hollis DR, Levine EG, Otterson GA, Millard F, and Ratain MJ (2007) A comparison of the pharmacokinetics and pharmacodynamics of docetaxel between African-American and Caucasian cancer patients: CALGB 9871. Clinical cancer research : an official journal of the American Association for Cancer Research 13:3302-3311.

Li C, Wang B, Lu D, Jin JY, Gao Y, Matsunaga K, Igawa Y, Nijem I, Lu M, Strasak A, Chernyukhin N, and Girish S (2016) Ethnic sensitivity assessment of the antibody-drug conjugate trastuzumab emtansine (T-DM1) in patients with HER2-positive locally advanced or metastatic breast cancer. Cancer Chemother Pharmacol 78:547-558.

Li H, Han TH, Hunder NN, Jang G, and Zhao B (2017a) Population Pharmacokinetics of Brentuximab Vedotin in Patients With CD30-Expressing Hematologic Malignancies. J Clin Pharmacol.

Li J, Al-Huniti N, Henningsson A, Tang W, and Masson E (2017b) Population pharmacokinetic and exposure simulation analysis for cediranib (AZD2171) in pooled Phase I/II studies in patients with cancer. Br J Clin Pharmacol.

Li J, Jameson MB, Baguley BC, Pili R, and Baker SD (2008) Population pharmacokinetic-pharmacodynamic model of the vascular-disrupting agent 5,6-dimethylxanthenone-4-acetic acid in cancer patients. Clinical cancer research : an official journal of the American Association for Cancer Research 14:2102-2110.

Li J, Karlsson MO, Brahmer J, Spitz A, Zhao M, Hidalgo M, and Baker SD (2006) CYP3A phenotyping approach to predict systemic exposure to EGFR tyrosine kinase inhibitors. Journal of the National Cancer Institute 98:1714-1723.

Liang S, Brundage RC, Jacobson PA, Blaes A, and Kirstein MN (2016) Pharmacokinetic-pharmacodynamic modelling of acute N-terminal pro B-type natriuretic peptide after doxorubicin infusion in breast cancer. Brit J Clin Pharmaco 82:773-783.

Lim CN and Salem AH (2015) A semi-mechanistic integrated pharmacokinetic/pharmacodynamic model of the testosterone effects of the gonadotropinreleasing hormone agonist leuprolide in prostate cancer patients. Clin Pharmacokinet 54:963-973.

Lim HS, Bae KS, Jung JA, Noh YH, Hwang AK, Jo YW, Hong YS, Kim K, Lee JL, Park SJ, Kim JE, Kang YK, and Kim TW (2015a) Predicting the efficacy of an oral paclitaxel formulation (DHP107) through modeling and simulation. Clin Ther 37:402-417.

Lim HS, Ryu KW, Lee JH, Kim YW, Ju Choi I, Kim MJ, Park YI, Hwang A, and Park SR (2015b) Postgastrectomy pharmacokinetic changes of S-1 in patients with localized advanced gastric cancer. J Clin Pharmacol 55:926-935.

Lindauer A, Di Gion P, Kanefendt F, Tomalik-Scharte D, Kinzig M, Rodamer M, Dodos F, Sorgel F, Fuhr U, and Jaehde U (2010a) Pharmacokinetic/pharmacodynamic modeling of biomarker response to sunitinib in healthy volunteers. Clin Pharmacol Ther 87:601-608.

Lindauer A, Eickhoff C, Kloft C, and Jaehde U (2010b) Population pharmacokinetics of high-dose carboplatin in children and adults. Ther Drug Monit 32:159-168 
Pharmacokinetic models for precision dosing in oncology - Supplementary appendix

Long-Boyle JR, Savic R, Yan S, Bartelink I, Musick L, French D, Law J, Horn B, Cowan MJ, and Dvorak CC (2015) Population Pharmacokinetics of Busulfan in Pediatric and Young Adult Patients Undergoing Hematopoietic Cell Transplant: A Model-Based Dosing Algorithm for Personalized Therapy and Implementation Into Routine Clinical Use. Ther Drug Monit 37:236-245.

Lu C, Suri A, Shyu WC, and Prakash S (2014a) Assessment of cytochrome P450-mediated drug-drug interaction potential of orteronel and exposure changes in patients with renal impairment using physiologically based pharmacokinetic modeling and simulation. Biopharm Drug Dispos 35:543-552.

Lu D, Girish S, Gao Y, Wang B, Yi JH, Guardino E, Samant M, Cobleigh M, Rimawi M, Conte P, and Jin JY (2014b) Population pharmacokinetics of trastuzumab emtansine (T-DM1), a HER2-targeted antibody-drug conjugate, in patients with HER2-positive metastatic breast cancer: clinical implications of the effect of covariates. Cancer Chemother Pharmacol 74:399-410.

Lu D, Joshi A, Wang B, Olsen S, Yi JH, Krop IE, Burris HA, and Girish S (2013) An integrated multiple-analyte pharmacokinetic model to characterize trastuzumab emtansine (T-DM1) clearance pathways and to evaluate reduced pharmacokinetic sampling in patients with HER2-positive metastatic breast cancer. Clin Pharmacokinet 52:657-672.

Lu JF, Claret L, Sutjandra L, Kuchimanchi M, Melara R, Bruno R, and Sun YN (2010) Population pharmacokinetic/pharmacodynamic modeling for the time course of tumor shrinkage by motesanib in thyroid cancer patients. Cancer Chemother Pharmacol 66:1151-1158.

Lu JF, Eppler SM, Wolf J, Hamilton M, Rakhit A, Bruno R, and Lum BL (2006) Clinical pharmacokinetics of erlotinib in patients with solid tumors and exposure-safety relationship in patients with non-small cell lung cancer. Clinical pharmacology and therapeutics 80:136-145.

Lu JF, Rasmussen E, Karlan BY, Vergote IB, Navale L, Kuchimanchi M, Melara R, Stepan DE, Weinreich DM, and Sun YN (2012) Exposure-response relationship of AMG 386 in combination with weekly paclitaxel in recurrent ovarian cancer and its implication for dose selection. Cancer Chemother Pharmacol 69:1135-1144.

Ma PM, Yang BB, Wang YM, Peterson M, Narayanan A, Sutjandra L, Rodriguez R, and Chow A (2009) Population Pharmacokinetic Analysis of Panitumumab in Patients With Advanced Solid Tumors. J Clin Pharmacol 49:1142-1156.

Maass C, Kletting P, Bunjes D, Mahren B, Beer AJ, and Glatting G (2015) Population-Based Modeling Improves Treatment Planning Before (90)YLabeled Anti-CD66 Antibody Radioimmunotherapy. Cancer biotherapy \& radiopharmaceuticals 30:285-290.

Makino Y, Watanabe M, Makihara RA, Nokihara H, Yamamoto N, Ohe Y, Sugiyama E, Sato H, and Hayashi Y (2016) Simultaneous optimization of limited sampling points for pharmacokinetic analysis of amrubicin and amrubicinol in cancer patients. Asia-Pacific journal of clinical oncology 12:259-264.

Mao J, Johnson TR, Shen Z, and Yamazaki S (2013) Prediction of crizotinib-midazolam interaction using the Simcyp population-based simulator: comparison of CYP3A time-dependent inhibition between human liver microsomes versus hepatocytes. Drug metabolism and disposition: the biological fate of chemicals 41:343-352.

Marachelian A, Desai A, Balis F, Katzenstein H, Qayed M, Armstrong M, Neville KA, Cohn SL, Bush M, Gunawan R, Lim AP, Smith MA, and Smith LM (2016) Comparative pharmacokinetics, safety, and tolerability of two sources of ch14.18 in pediatric patients with high-risk neuroblastoma following myeloablative therapy. Cancer Chemother Pharmacol 77:405-412. 
Pharmacokinetic models for precision dosing in oncology - Supplementary appendix

Marostica E, Sukbuntherng J, Loury D, de Jong J, de Trixhe XW, Vermeulen A, De Nicolao G, O'Brien S, Byrd JC, Advani R, McGreivy J, and Poggesi I (2015) Population pharmacokinetic model of ibrutinib, a Bruton tyrosine kinase inhibitor, in patients with B cell malignancies. Cancer Chemother Pharmacol 75:111-121.

Masumori N, Kunishima Y, Hirobe M, Takeuchi M, Takayanagi A, Tsukamoto T, and Itoh T (2008) Measurement of plasma concentration of gemcitabine and its metabolite dFdU in hemodialysis patients with advanced urothelial cancer. Jpn J Clin Oncol 38:182-185.

McCormack P and Sapunar F (2008) Pharmacokinetic profile of the fulvestrant loading dose regimen in postmenopausal women with hormone receptor-positive advanced breast cancer. Clin Breast Cancer 8:347-351.

McCune JS, Batchelder A, Guthrie KA, Witherspoon R, Appelbaum FR, Phillips B, Vicini P, Salinger DH, and McDonald GB (2009a) Personalized dosing of cyclophosphamide in the total body irradiation-cyclophosphamide conditioning regimen: a phase II trial in patients with hematologic malignancy. Clin Pharmacol Ther 85:615-622.

McCune JS, Bemer MJ, Barrett JS, Scott Baker K, Gamis AS, and Holford NH (2014) Busulfan in infant to adult hematopoietic cell transplant recipients: a population pharmacokinetic model for initial and Bayesian dose personalization. Clin Cancer Res 20:754-763.

McCune JS, Salinger DH, Vicini P, Oglesby C, Blough DK, and Park JR (2009b) Population Pharmacokinetics of Cyclophosphamide and Metabolites in Children With Neuroblastoma: A Report From the Children's Oncology Group. J Clin Pharmacol 49:88-102.

McDonald GB, McCune JS, Batchelder A, Cole S, Phillips B, Ren AG, Vicini P, Witherspoon R, Kalhorn TF, and Slattery JT (2005) Metabolism-based cyclophosphamide dosing for hematopoietic cell transplant. Clin Pharmacol Ther 78:298-308.

Meille C, Barbolosi D, Ciccolini J, Freyer G, and Iliadis A (2016) Revisiting Dosing Regimen Using Pharmacokinetic/Pharmacodynamic Mathematical Modeling: Densification and Intensification of Combination Cancer Therapy. Clin Pharmacokinet 55:1015-1025.

Menon-Andersen D, Mondick JT, Jayaraman B, Thompson PA, Blaney SM, Bernstein M, Bond M, Champagne M, Fossler MJ, and Barrett JS (2009) Population pharmacokinetics of imatinib mesylate and its metabolite in children and young adults. Cancer Chemother Pharmacol 63:229-238.

Merino-Sanjuan M, Monteiro JF, Porta-Oltra B, Maestu I, Almenar D, and Jimenez-Torres NV (2011) Effect of age on systemic exposure and haematological toxicity of carboplatin in advanced non-small cell lung cancer patients. Basic Clin Pharmacol Toxicol 109:457-464.

Miguel-Lillo B, Valenzuela B, Peris-Ribera JE, Soto-Matos A, and Perez-Ruixo JJ (2015) Population pharmacokinetics of kahalalide F in advanced cancer patients. Cancer Chemother Pharmacol 76:365-374.

Miles D, Jumbe NL, Lacy S, and Nguyen L (2016a) Population Pharmacokinetic Model of Cabozantinib in Patients with Medullary Thyroid Carcinoma and Its Application to an Exposure-Response Analysis. Clin Pharmacokinet 55:93-105.

Miles DR, Wada DR, Jumbe NL, Lacy SA, and Nguyen LT (2016b) Population pharmacokinetic/pharmacodynamic modeling of tumor growth kinetics in medullary thyroid cancer patients receiving cabozantinib. Anti-cancer drugs 27:328-341.

Miller VA, Rigas JR, Tong WP, Reid JR, Pisters KMW, Grant SC, Heelan RT, and Kris MG (1997) Phase II trial of chloroquinoxaline sulfonamide (CQS) in patients with stage III and IV non-small-cell lung cancer. Cancer Chemother Pharmacol 40:415-418.

Millward MJ, Newell DR, Yuen K, Matthews JP, Balmanno K, Charlton CJ, Gumbrell L, Lind MJ, Chapman F, Proctor M, and et al. (1995) Pharmacokinetics and pharmacodynamics of prolonged oral etoposide in women with metastatic breast cancer. Cancer Chemother Pharmacol 37:161-167. 
Pharmacokinetic models for precision dosing in oncology - Supplementary appendix

Minami H, Kawada K, Sasaki Y, Tahara M, Igarashi T, Itoh K, Fujii H, Saeki T, Ozawa K, and Sato H (2009) Population pharmacokinetics of docetaxel in patients with hepatic dysfunction treated in an oncology practice. Cancer science 100:144-149.

Minami H, Ohe Y, Niho S, Goto K, Ohmatsu H, Kubota K, Kakinuma R, Nishiwaki Y, Nokihara H, Sekine I, Saijo N, Hanada K, and Ogata H (2004) Comparison of pharmacokinetics and pharmacodynamics of docetaxel and Cisplatin in elderly and non-elderly patients: why is toxicity increased in elderly patients? Journal of clinical oncology : official journal of the American Society of Clinical Oncology 22:2901-2908.

Mittapalli RK, Nuthalapati S, Shepherd SP, and Xiong H (2017) Population pharmacokinetics of ABT-767 in BRCA1 or BRCA2 mutation carriers with advanced solid tumors or in subjects with high grade serous ovarian, primary peritoneal or fallopian tube cancer. Cancer Chemother Pharmacol 79:587-594.

Mizuno T, Fukuda T, Fouladi M, Blaney SM, Perentesis JP, and Vinks AA (2014) Population Pharmacokinetic Analysis of Temsirolimus in Children. Clinical pharmacology and therapeutics 95:S98-S98.

Monjanel-Mouterde S, Lejeune C, Ciccolini J, Merite N, Hadjaj D, Bonnier P, Piana P, and Durand A (2002) Bayesian population model of methotrexate to guide dosage adjustments for folate rescue in patients with breast cancer. J Clin Pharm Ther 27:189-195.

Montazeri A, Boucaud M, Lokiec F, Pinguet F, Culine S, Deporte-Fety R, Albin N, Laguerre B, Goupil A, Bugat R, Canal P, and Chatelut E (2000) Population pharmacokinetics of topotecan: intraindividual variability in total drug. Cancer Chemother Pharmacol 46:375-381.

Montazeri A, Culine S, Laguerre B, Pinguet F, Lokiec F, Albin N, Goupil A, Deporte-Fety R, Bugat R, Canal P, and Chatelut E (2002) Individual adaptive dosing of topotecan in ovarian cancer. Clin Cancer Res 8:394-399.

Moore AS, Norris R, Price G, Nguyen T, Ni M, George R, van Breda K, Duley J, Charles B, and Pinkerton R (2011) Vincristine pharmacodynamics and pharmacogenetics in children with cancer: a limited-sampling, population modelling approach. Journal of paediatrics and child health 47:875882.

Mougenot P, Pinguet F, Fabbro M, Culine S, Poujol S, Astre C, and Bressolle F (2004) Population pharmacokinetics of melphalan, infused over a 24hour period, in patients with advanced malignancies. Cancer Chemother Pharmacol 53:503-512.

Mould DR, Holford NH, Schellens JH, Beijnen JH, Hutson PR, Rosing H, ten Bokkel Huinink WW, Rowinsky EK, Schiller JH, Russo M, and Ross G (2002) Population pharmacokinetic and adverse event analysis of topotecan in patients with solid tumors. Clinical pharmacology and therapeutics 71:334-348.

Mross K, Hollander N, Hauns B, Schumacher M, and Maier-Lenz H (2000) The pharmacokinetics of a 1-h paclitaxel infusion. Cancer Chemother Pharmacol 45:463-470.

Nader A, Zahran N, Alshammaa A, Altaweel H, Kassem N, and Wilby KJ (2017) Population Pharmacokinetics of Intravenous Methotrexate in Patients with Hematological Malignancies: Utilization of Routine Clinical Monitoring Parameters. European journal of drug metabolism and pharmacokinetics 42:221-228.

Nagai N, Ogata H, Wada Y, Tsujino D, Someya K, Ohno T, Masuhara K, Tanaka Y, Takahashi H, Nagai H, Kato K, Koshiba Y, Igarashi T, Yokoyama A, Kinameri K, Kato T, and Kurita Y (1998) Population pharmacokinetics and pharmacodynamics of cisplatin in patients with cancer: analysis with the NONMEM program. J Clin Pharmacol 38:1025-1034. 
Pharmacokinetic models for precision dosing in oncology - Supplementary appendix

Nagulu M, Kiran VU, Nalini Y, Reddy YN, and Krishna DR (2010) Population pharmacokinetics of methotrexate in Indian cancer patients. Asian Pacific journal of cancer prevention: APJCP 11:403-407.

Nakamura H, Sato T, Okada K, Miura G, Ariyoshi N, Nakazawa K, and Kitada M (2008) Population pharmacokinetics of oral busulfan in young Japanese children before hematopoietic stem cell transplantation. Ther Drug Monit 30:75-83.

Nakashima H, Lieberman R, Karato A, Arioka H, Ohmatsu H, Nomura N, Shiraishi J, Tamura T, Eguchi K, Shinkai T, Sasaki Y, Yamamoto N, Hukuda M, Oshita F, Ohe Y, and Saijo N (1995) Efficient Sampling Strategies for Forecasting Pharmacokinetic Parameters of Irinotecan (Cpt-11) Implication for Area under the Concentration-Time Curve Monitoring. Ther Drug Monit 17:221-229.

Nalda-Molina R, Valenzuela B, Ramon-Lopez A, Miguel-Lillo B, Soto-Matos A, and Perez-Ruixo JJ (2009) Population pharmacokinetics meta-analysis of plitidepsin (Aplidin) in cancer subjects. Cancer Chemother Pharmacol 64:97-108.

Narayanan R, Hoffmann M, Kumar G, and Surapaneni S (2016) Application of a "Fit for Purpose" PBPK Model to Investigate the CYP3A4 Induction Potential of Enzalutamide. Drug metabolism letters 10:172-179.

Nath CE, Shaw PJ, Montgomery K, and Earl JW (2005) Melphalan pharmacokinetics in children with malignant disease: influence of body weight, renal function, carboplatin therapy and total body irradiation. Br J Clin Pharmacol 59:314-324.

Nath CE, Shaw PJ, Montgomery K, and Earl JW (2007) Population pharmacokinetics of melphalan in paediatric blood or marrow transplant recipients. Br J Clin Pharmacol 64:151-164.

Nath CE, Shaw PJ, Trotman J, Zeng L, Duffull SB, Hegarty G, McLachlan AJ, Gurney H, Kerridge I, Kwan YL, Presgrave P, Tiley C, Joshua D, and Earl J (2010) Population pharmacokinetics of melphalan in patients with multiple myeloma undergoing high dose therapy. Br J Clin Pharmacol 69:484-497.

Neely M, Philippe M, Rushing T, Fu X, van Guilder M, Bayard D, Schumitzky A, Bleyzac N, and Goutelle S (2016) Accurately Achieving Target Busulfan Exposure in Children and Adolescents With Very Limited Sampling and the BestDose Software. Ther Drug Monit 38:332-342.

Neville K, Blaney S, Bernstein M, Thompson P, Adams D, Aleksic A, and Berg S (2004) Pharmacokinetics of O(6)-benzylguanine in pediatric patients with central nervous system tumors: a pediatric oncology group study. Clinical cancer research : an official journal of the American Association for Cancer Research 10:5072-5075.

Ng CM, Lum BL, Gimenez V, Kelsey S, and Allison D (2006) Rationale for fixed dosing of pertuzumab in cancer patients based on population pharmacokinetic analysis. Pharm Res 23:1275-1284.

Ng CM, Patnaik A, Beeram M, Lin CC, and Takimoto CH (2011) Mechanism-based pharmacokinetic/pharmacodynamic model for troxacitabineinduced neutropenia in cancer patients. Cancer Chemother Pharmacol 67:985-994.

Nguyen L, Leger F, Lennon S, and Puozzo C (2006) Intravenous busulfan in adults prior to haematopoietic stem cell transplantation: a population pharmacokinetic study. Cancer Chemother Pharmacol 57:191-198.

Nikanjam M, Stewart CF, Takimoto CH, Synold TW, Beaty O, Fouladi M, and Capparelli EV (2015) Population pharmacokinetic analysis of oxaliplatin in adults and children identifies important covariates for dosing. Cancer Chemother Pharmacol 75:495-503. 
Pharmacokinetic models for precision dosing in oncology - Supplementary appendix

Niu J, Scheuerell C, Mehrotra S, Karan S, Puhalla S, Kiesel BF, Ji J, Chu E, Gopalakrishnan M, Ivaturi V, Gobburu J, and Beumer JH (2017) ParentMetabolite Pharmacokinetic Modeling and Pharmacodynamics of Veliparib (ABT-888), a PARP Inhibitor, in Patients With BRCA 1/2-Mutated Cancer or PARP-Sensitive Tumor Types. J Clin Pharmacol.

Noh YH, Ko YJ, Cho SH, Ghim JL, Choe S, Jung JA, Kim UJ, Jin SJ, Park HJ, Song GS, Lim HS, and Bae KS (2012) Pharmacokinetic comparison of 2 formulations of anastrozole $(1 \mathrm{mg})$ in healthy Korean male volunteers: a randomized, single-dose, 2-period, 2-sequence, crossover study. Clin Ther 34:305-313.

Noh YH, Lim HS, Jung JA, Song TH, and Bae KS (2015) Population pharmacokinetics of HM781-36 (poziotinib), pan-human EGF receptor (HER) inhibitor, and its two metabolites in patients with advanced solid malignancies. Cancer Chemother Pharmacol 75:97-109.

Onoue H, Yano I, Tanaka A, Itohara K, Hanai A, Ishiguro H, Motohashi H, Masuda S, and Matsubara K (2016) Significant effect of age on docetaxel pharmacokinetics in Japanese female breast cancer patients by using the population modeling approach. Eur J Clin Pharmacol 72:703-710.

Ou Y, Doshi S, Nguyen A, Jonsson F, Aggarwal S, Rajangam K, Dimopoulos MA, Stewart AK, Badros A, Papadopoulos KP, Siegel D, Jagannath S, Vij R, Niesvizky R, Graham R, and Visich J (2017) Population Pharmacokinetics and Exposure-Response Relationship of Carfilzomib in Patients With Multiple Myeloma. J Clin Pharmacol 57:663-677.

Ouellet D, Periclou AP, Johnson RD, Woodworth JR, and Lalonde RL (2000) Population pharmacokinetics of pemetrexed disodium (ALIMTA) in patients with cancer. Cancer Chemother Pharmacol 46:227-234.

Ozawa K, Minami H, and Sato H (2007) Population pharmacokinetic and pharmacodynamic analysis for time courses of docetaxel-induced neutropenia in Japanese cancer patients. Cancer science 98:1985-1992.

Pahlka RB and Sonnad JR (2006) The effects of dialysis on I-131 kinetics and dosimetry in thyroid cancer patients - A pharmacokinetic model. Health Phys 91:227-237.

Panetta JC, Kirstein MN, Gajjar A, Nair G, Fouladi M, Heideman RL, Wilkinson M, and Stewart CF (2003) Population pharmacokinetics of temozolomide and metabolites in infants and children with primary central nervous system tumors. Cancer Chemother Pharmacol 52:435-441.

Panoilia E, Schindler E, Samantas E, Aravantinos G, Kalofonos HP, Christodoulou C, Patrinos GP, Friberg LE, and Sivolapenko G (2015) A pharmacokinetic binding model for bevacizumab and VEGF165 in colorectal cancer patients. Cancer Chemother Pharmacol 75:791-803.

Panteix G, Beaujard A, Garbit F, Chaduiron-Faye C, Guillaumont M, Gilly F, Baltassat P, and Bressolle F (2002) Population pharmacokinetics of cisplatin in patients with advanced ovarian cancer during intraperitoneal hyperthermia chemotherapy. Anticancer Res 22:1329-1336.

Parra-Guillen ZP, Cendros Carreras JM, Peraire C, Obach R, Prunynosa J, Chetaille E, and Troconiz IF (2015) Population pharmacokinetic modelling of irosustat in postmenopausal women with oestrogen-receptor positive breast cancer incorporating non-linear red blood cell uptake. Pharm Res 32:1493-1504.

Parrott NJ, Yu LJ, Takano R, Nakamura M, and Morcos PN (2016) Physiologically Based Absorption Modeling to Explore the Impact of Food and Gastric pH Changes on the Pharmacokinetics of Alectinib. AAPS J 18:1464-1474.

Patel YT, Daryani VM, Patel P, Zhou D, Fangusaro J, Carlile DJ, Martin PD, Aarons L, and Stewart CF (2017) Population Pharmacokinetics of Selumetinib and Its Metabolite N-desmethyl-selumetinib in Adult Patients With Advanced Solid Tumors and Children With Low-Grade Gliomas. CPT: pharmacometrics \& systems pharmacology. 
Pharmacokinetic models for precision dosing in oncology - Supplementary appendix

Paz-Ares LG, Gomez-Roca C, Delord JP, Cervantes A, Markman B, Corral J, Soria JC, Berge Y, Roda D, Russell-Yarde F, Hollingsworth S, Baselga J, Umana P, Manenti L, and Tabernero J (2011) Phase I pharmacokinetic and pharmacodynamic dose-escalation study of RG7160 (GA201), the first glycoengineered monoclonal antibody against the epidermal growth factor receptor, in patients with advanced solid tumors. J Clin Oncol 29:3783-3790.

Peer CJ, Goey AK, Sissung TM, Erlich S, Lee MJ, Tomita Y, Trepel JB, Piekarz R, Balasubramaniam S, Bates SE, and Figg WD (2016) UGT1A1 genotype-dependent dose adjustment of belinostat in patients with advanced cancers using population pharmacokinetic modeling and simulation. J Clin Pharmacol 56:450-460.

Pelsor FR, Allen LM, and Creaven PJ (1978) Multicompartment pharmacokinetic model of 4'-demethylepipodophyllotoxin 9-(4,6-O-ethylidene-beta-Dglucopyranoside) in humans. J Pharm Sci 67:1106-1108.

Peng B, Boddy AV, Cole M, Pearson AD, Chatelut E, Rubie H, and Newell DR (1995) Comparison of methods for the estimation of carboplatin pharmacokinetics in paediatric cancer patients. Eur J Cancer 31A:1804-1810.

Perez-Ruixo JJ, Zannikos P, Hirankarn S, Stuyckens K, Ludwig EA, Soto-Matos A, Lopez-Lazaro L, and Owen JS (2007) Population pharmacokinetic meta-analysis of trabectedin (ET-743, Yondelis) in cancer patients. Clin Pharmacokinet 46:867-884.

Perez Ruixo JJ, Doshi S, Sohn W, and Chow A (2015) Quantitative pharmacology of denosumab in patients with bone metastases from solid tumors. $J$ Clin Pharmacol 55 Suppl 3:S85-92.

Petain A, Kattygnarath D, Azard J, Chatelut E, Delbaldo C, Geoerger B, Barrois M, Seronie-Vivien S, LeCesne A, Vassal G, and Innovative Therapies with Children with Cancer European c (2008) Population pharmacokinetics and pharmacogenetics of imatinib in children and adults. Clin Cancer Res 14:7102-7109.

Piergies AA and Benson AB, 3rd (1995) Pharmacokinetics and acute cardiovascular effects of menogaril in patients with metastatic tumors. Int J Clin Pharmacol Ther 33:63-69.

Pignon T, Lacarelle B, Duffaud F, Guillet P, Catalin J, Durand A, and Favre R (1995) Dosage Adjustment of High-Dose Methotrexate Using BayesianEstimation - a Comparative-Study of 2 Different Concentrations at the End of 8-H Infusions. Ther Drug Monit 17:471-478.

Pignon T, Lacarelle B, Duffaud F, Guillet P, Catalin J, Durand A, Monjanel S, and Favre R (1994) Pharmacokinetics of high-dose methotrexate in adult osteogenic sarcoma. Cancer Chemother Pharmacol 33:420-424.

Pointreau Y, Azzopardi N, Ternant D, Calais G, and Paintaud G (2016) Cetuximab Pharmacokinetics Influences Overall Survival in Patients With Head and Neck Cancer. Therapeutic drug monitoring 38:567-572.

Posada MM, Bacon JA, Schneck KB, Tirona RG, Kim RB, Higgins JW, Pak YA, Hall SD, and Hillgren KM (2015) Prediction of renal transporter mediated drug-drug interactions for pemetrexed using physiologically based pharmacokinetic modeling. Drug Metab Dispos 43:325-334.

Poujol S, Pinguet F, Ychou M, Abderrahim AG, Duffour J, and Bressolle FMM (2007) A limited sampling strategy to estimate the pharmacokinetic parameters of irinotecan and its active metabolite, SN-38, in patients with metastatic digestive cancer receiving the FOLFIRI regimen. Oncol Rep 18:1613-1621.

Puozzo C and Gridelli C (2004) Non-small-cell lung cancer in elderly patients: influence of age on vinorelbine oral pharmacokinetics. Clinical lung cancer 5:237-242. 
Pharmacokinetic models for precision dosing in oncology - Supplementary appendix

Quartino AL, Hillenbach C, Li J, Li H, Wada RD, Visich J, Li C, Heinzmann D, Jin JY, and Lum BL (2016) Population pharmacokinetic and exposureresponse analysis for trastuzumab administered using a subcutaneous "manual syringe" injection or intravenously in women with HER2positive early breast cancer. Cancer Chemother Pharmacol 77:77-88.

Quartino AL, Li H, Jin JY, Wada DR, Benyunes MC, McNally V, Vigano L, Nijem I, Lum BL, and Garg A (2017) Pharmacokinetic and exposureresponse analyses of pertuzumab in combination with trastuzumab and docetaxel during neoadjuvant treatment of HER2+ early breast cancer. Cancer Chemother Pharmacol 79:353-361.

Rahman A, Treat J, Roh JK, Potkul LA, Alvord WG, Forst D, and Woolley PV (1990) A Phase-I Clinical-Trial and Pharmacokinetic Evaluation of Liposome-Encapsulated Doxorubicin. Journal of clinical oncology : official journal of the American Society of Clinical Oncology 8:1093-1100.

Reece PA, Stafford I, Davy M, and Freeman S (1987) Disposition of unchanged cisplatin in patients with ovarian cancer. Clinical pharmacology and therapeutics 42:320-325.

Reid JM, Pendergrass TW, Krailo MD, Hammond GD, and Ames MM (1990) Plasma pharmacokinetics and cerebrospinal fluid concentrations of idarubicin and idarubicinol in pediatric leukemia patients: a Childrens Cancer Study Group report. Cancer research 50:6525-6528.

Reilly RM, Kirsh J, Gallinger S, Thiessen JJ, Damani M, Hay K, Polihronis J, Schmocker B, Odze R, Houle S, and et al. (1993) Compartmental analysis of the pharmacokinetics of radioiodinated monoclonal antibody B72.3 in colon cancer patients. Nuclear medicine and biology 20:5764.

Rezai K, Urien S, Isambert N, Roche H, Dieras V, Berille J, Bonneterre J, Brain E, and Lokiec F (2011) Pharmacokinetic evaluation of the vinorelbinelapatinib combination in the treatment of breast cancer patients. Cancer Chemother Pharmacol 68:1529-1536.

Richard B, Launay-Iliadis MC, Iliadis A, Just-Landi S, Blaise D, Stoppa AM, Viens P, Gaspard MH, Maraninchi D, Cano JP, and et al. (1992) Pharmacokinetics of mitoxantrone in cancer patients treated by high-dose chemotherapy and autologous bone marrow transplantation. $\mathrm{Br} J$ Cancer 65:399-404.

Robertson JF, Erikstein B, Osborne KC, Pippen J, Come SE, Parker LM, Gertler S, Harrison MP, and Clarke DA (2004) Pharmacokinetic profile of intramuscular fulvestrant in advanced breast cancer. Clin Pharmacokinet 43:529-538.

Robinson PJ, Bass L, Pond SM, Roberts MS, and Wagner JG (1988) Clinical applicability of current pharmacokinetic models: splanchnic elimination of 5-fluorouracil in cancer patients. J Pharmacokinet Biopharm 16:229-249.

Rodriguez-Vera L, Ramos-Suzarte M, Fernandez-Sanchez E, Soriano JL, Guitart CP, Hernandez GC, Jacobo-Cabral CO, de Castro Suarez N, and Codina HC (2015) Semimechanistic model to characterize nonlinear pharmacokinetics of nimotuzumab in patients with advanced breast cancer. J Clin Pharmacol 55:888-898.

Romero E, Velez de Mendizabal N, Cendros JM, Peraire C, Bascompta E, Obach R, and Troconiz IF (2012) Pharmacokinetic/pharmacodynamic model of the testosterone effects of triptorelin administered in sustained release formulations in patients with prostate cancer. J Pharmacol Exp Ther 342:788-798.

Rubin E, Wood V, Bharti A, Trites D, Lynch C, Hurwitz S, Bartel S, Levy S, Rosowsky A, Toppmeyer D, and et al. (1995) A phase I and pharmacokinetic study of a new camptothecin derivative, 9-aminocamptothecin. Clinical cancer research : an official journal of the American Association for Cancer Research 1:269-276. 
Pharmacokinetic models for precision dosing in oncology - Supplementary appendix

Rump AF, Botvinik-Helling S, Theisohn M, Biederbick W, Schierholz JM, Stemmler M, Fischbach R, Heindel W, Lackner K, and Klaus W (1996) Pharmacokinetics of intraarterial mitomycin $\mathrm{C}$ in the chemoembolisation treatment of liver metastases. General pharmacology 27:669-671.

Saba NF, Hurwitz SJ, Magliocca K, Kim S, Owonikoko TK, Harvey D, Ramalingam SS, Chen Z, Rogerio J, Mendel J, Kono SA, Lewis C, Chen AY, Higgins K, El-Deiry M, Wadsworth T, Beitler JJ, Shin DM, Sun SY, and Khuri FR (2014) Phase 1 and pharmacokinetic study of everolimus in combination with cetuximab and carboplatin for recurrent/metastatic squamous cell carcinoma of the head and neck. Cancer 120:3940-3951.

Sabot C, Marquet P, Debord J, Carpentier N, Merle L, and Lachatre G (1998) Bayesian pharmacokinetic estimation of vinorelbine in non-small-cell lung cancer patients. Eur J Clin Pharmacol 54:171-175.

Salas S, Mercier C, Ciccolini J, Pourroy B, Fanciullino R, Tranchand B, Monjanel-Mouterde S, Baciuchka-Palmaro M, Dupuis C, Yang C, Balti M, Lacarelle B, Duffaud F, Durand A, and Favre R (2006) Therapeutic drug monitoring for dose individualization of Cisplatin in testicular cancer patients based upon total platinum measurement in plasma. Therapeutic drug monitoring 28:532-539.

Salem AH, Koenig D, and Carlson D (2014) Pooled population pharmacokinetic analysis of phase I, II and III studies of linifanib in cancer patients. Clin Pharmacokinet 53:347-359.

Salinger DH, McCune JS, Ren AG, Shen DD, Slattery JT, Phillips B, McDonald GB, and Vicini P (2006) Real-time dose adjustment of cyclophosphamide in a preparative regimen for hematopoietic cell transplant: a Bayesian pharmacokinetic approach. Clin Cancer Res 12:48884898.

Salphati L, Pang J, Plise EG, Lee LB, Olivero AG, Prior WW, Sampath D, Wong S, and Zhang X (2012) Preclinical assessment of the absorption and disposition of the phosphatidylinositol 3-kinase/mammalian target of rapamycin inhibitor GDC-0980 and prediction of its pharmacokinetics and efficacy in human. Drug Metab Dispos 40:1785-1796.

Sandstrom M, Freijs A, Larsson R, Nygren P, Fjallskog ML, Bergh J, and Karlsson MO (1996) Lack of relationship between systemic exposure for the component drugs of the fluorouracil, epirubicin, and 4-hydroxycyclophosphamide regimen in breast cancer patients. Journal of clinical oncology : official journal of the American Society of Clinical Oncology 14:1581-1588.

Sandstrom M, Lindman H, Nygren P, Johansson M, Bergh J, and Karlsson MO (2006) Population analysis of the pharmacokinetics and the haematological toxicity of the fluorouracil-epirubicin-cyclophosphamide regimen in breast cancer patients. Cancer Chemother Pharmacol 58:143-156.

Sassen SD, Mathot RA, Pieters R, Kloos RQ, de Haas V, Kaspers GJ, van den Bos C, Tissing WJ, Te Loo M, Bierings MB, Kollen WJ, Zwaan CM, and van der Sluis IM (2017) Population pharmacokinetics of intravenous Erwinia asparaginase in pediatric acute lymphoblastic leukemia patients. Haematologica 102:552-561.

Schaiquevich P, Panetta JC, Iacono LC, Freeman BB, 3rd, Santana VM, Gajjar A, and Stewart CF (2007) Population pharmacokinetic analysis of topotecan in pediatric cancer patients. Clin Cancer Res 13:6703-6711.

Schmidli H, Peng B, Riviere GJ, Capdeville R, Hensley M, Gathmann I, Bolton AE, Racine-Poon A, and Group IS (2005) Population pharmacokinetics of imatinib mesylate in patients with chronic-phase chronic myeloid leukaemia: results of a phase III study. Br J Clin Pharmacol 60:35-44.

Sethi VS, Jackson DV, Jr., White DR, Richards F, 2nd, Stuart JJ, Muss HB, Cooper MR, and Spurr CL (1981) Pharmacokinetics of vincristine sulfate in adult cancer patients. Cancer research 41:3551-3555. 
Pharmacokinetic models for precision dosing in oncology - Supplementary appendix

Sharma S, Mittapalli RK, Holen KD, and Xiong H (2015) Population Pharmacokinetics of ABT-806, an Investigational Anti-Epidermal Growth Factor Receptor (EGFR) Monoclonal Antibody, in Advanced Solid Tumor Types Likely to Either Over-Express Wild-Type EGFR or Express Variant III Mutant EGFR. Clin Pharmacokinet 54:1071-1081.

Shen MY, Schilder RJ, Obasaju C, and Gallo JM (2002) Population pharmacokinetic and limited sampling models for carboplatin administered in highdose combination regimens with peripheral blood stem cell support. Cancer Chemother Pharmacol 50:243-250.

Shi JG, Bowman KJ, Chen X, Maleski J, Leopold L, and Yeleswaram S (2017) Population Pharmacokinetic and Pharmacodynamic Modeling of Epacadostat in Patients With Advanced Solid Malignancies. J Clin Pharmacol 57:720-729.

Singh AP and Shah DK (2017) Application of a PK-PD Modeling and Simulation-Based Strategy for Clinical Translation of Antibody-Drug Conjugates: a Case Study with Trastuzumab Emtansine (T-DM1). AAPS J.

Singh RP, Patel B, Kallender H, Ottesen LH, Adams LM, and Cox DS (2015) Population pharmacokinetics modeling and analysis of foretinib in adult patients with advanced solid tumors. J Clin Pharmacol 55:1184-1192.

Slaviero KA, Clarke SJ, McLachlan AJ, Blair EY, and Rivory LP (2004) Population pharmacokinetics of weekly docetaxel in patients with advanced cancer. Br J Clin Pharmacol 57:44-53.

Smith DC, Jodrell DI, Egorin MJ, Ambinder RM, Zuhowski EG, Kreis W, Ellis PG, and Trump DL (1993) Phase II trial and pharmacokinetic assessment of intravenous melphalan in patients with advanced prostate cancer. Cancer Chemother Pharmacol 31:363-368.

Soto E, Keizer RJ, Troconiz IF, Huitema AD, Beijnen JH, Schellens JH, Wanders J, Cendros JM, Obach R, Peraire C, Friberg LE, and Karlsson MO (2011a) Predictive ability of a semi-mechanistic model for neutropenia in the development of novel anti-cancer agents: two case studies. Investigational new drugs 29:984-995.

Soto E, Staab A, Doege C, Freiwald M, Munzert G, and Troconiz IF (2011b) Comparison of different semi-mechanistic models for chemotherapyrelated neutropenia: application to BI 2536 a Plk-1 inhibitor. Cancer Chemother Pharmacol 68:1517-1527.

Soto E, Staab A, Tillmann C, Trommeshauser D, Fritsch H, Munzert G, and Troconiz IF (2010) Semi-mechanistic population pharmacokinetic/pharmacodynamic model for neutropenia following therapy with the Plk-1 inhibitor BI 2536 and its application in clinical development. Cancer Chemother Pharmacol 66:785-795.

Stefanini MO, Wu FT, Mac Gabhann F, and Popel AS (2010) Increase of plasma VEGF after intravenous administration of bevacizumab is predicted by a pharmacokinetic model. Cancer research 70:9886-9894.

Stuyckens K, Saad F, Xu XS, Ryan CJ, Smith MR, Griffin TW, Yu MK, Vermeulen A, Nandy P, and Poggesi I (2014) Population pharmacokinetic analysis of abiraterone in chemotherapy-naive and docetaxel-treated patients with metastatic castration-resistant prostate cancer. Clin Pharmacokinet 53:1149-1160.

Sugiyama E, Kaniwa N, Kim SR, Hasegawa R, Saito Y, Ueno H, Okusaka T, Ikeda M, Morizane C, Kondo S, Yamamoto N, Tamura T, Furuse J, Ishii H, Yoshida T, Saijo N, and Sawada JI (2010) Population Pharmacokinetics of Gemcitabine and Its Metabolite in Japanese Cancer Patients Impact of Genetic Polymorphisms. Clin Pharmacokinet 49:549-558. 
Pharmacokinetic models for precision dosing in oncology - Supplementary appendix

Sun W, O'Dwyer PJ, Finn RS, Ruiz-Garcia A, Shapiro GI, Schwartz GK, DeMichele A, and Wang D (2017) Characterization of Neutropenia in Advanced Cancer Patients Following Palbociclib Treatment Using a Population Pharmacokinetic-Pharmacodynamic Modeling and Simulation Approach. J Clin Pharmacol.

Suttle AB, de Souza P, and Arumugham T (2013) Bayesian methods for pharmacokinetic/pharmacodynamic modeling of pazopanib-induced increases in blood pressure and transaminases. J Clin Pharmacol 53:377-384.

Tan W, Yamazaki S, Johnson TR, Wang R, O'Gorman MT, Kirkovsky L, Boutros T, Brega NM, and Bello A (2017) Effects of Renal Function on Crizotinib Pharmacokinetics: Dose Recommendations for Patients with ALK-Positive Non-Small Cell Lung Cancer. Clinical drug investigation 37:363-373.

Tanaka C, O'Reilly T, Kovarik JM, Shand N, Hazell K, Judson I, Raymond E, Zumstein-Mecker S, Stephan C, Boulay A, Hattenberger M, Thomas G, and Lane HA (2008) Identifying optimal biologic doses of everolimus (RAD001) in patients with cancer based on the modeling of preclinical and clinical pharmacokinetic and pharmacodynamic data. J Clin Oncol 26:1596-1602.

Tanii H, Shitara Y, and Horie T (2011) Population pharmacokinetic analysis of letrozole in Japanese postmenopausal women. Eur J Clin Pharmacol 67:1017-1025.

Taylor CW, Salmon SE, Satterlee WG, Robertone AB, McCloskey TM, Holdsworth MT, Plezia PM, and Alberts DS (1990) A phase I and pharmacokinetic study of intravenous vinzolidine. Investigational new drugs 8 Suppl 1:S51-57.

ter Heine R, Binkhorst L, de Graan AJ, de Bruijn P, Beijnen JH, Mathijssen RH, and Huitema AD (2014) Population pharmacokinetic modelling to assess the impact of CYP2D6 and CYP3A metabolic phenotypes on the pharmacokinetics of tamoxifen and endoxifen. Br J Clin Pharmacol 78:572-586.

Thai HT, Mazuir F, Cartot-Cotton S, and Veyrat-Follet C (2015) Optimizing pharmacokinetic bridging studies in paediatric oncology using physiologically-based pharmacokinetic modelling: application to docetaxel. Br J Clin Pharmacol 80:534-547.

Thai HT, Veyrat-Follet C, Mentre F, and Comets E (2013) Population pharmacokinetic analysis of free and bound aflibercept in patients with advanced solid tumors. Cancer Chemother Pharmacol 72:167-180.

Thai HT, Veyrat-Follet C, Vivier N, Dubruc C, Sanderink G, Mentre F, and Comets E (2011) A mechanism-based model for the population pharmacokinetics of free and bound aflibercept in healthy subjects. Br J Clin Pharmacol 72:402-414.

Tham LS, Wang LZ, Soo RA, Lee SC, Lee HS, Yong WP, Goh BC, and Holford NHG (2008) A pharmacodynamic model for the time course of tumor shrinkage by gemcitabine plus carboplatin in non-small cell lung cancer patients. Clinical cancer research : an official journal of the American Association for Cancer Research 14:4213-4218.

Thompson P, Balis F, Serabe BM, Berg S, Adamson P, Klenke R, Aiken A, Packer R, Murry DJ, Jakacki R, and Blaney SM (2003) Pharmacokinetics of phenylacetate administered as a 30-min infusion in children with refractory cancer. Cancer Chemother Pharmacol 52:417-423.

Thompson PA, Gupta M, Rosner GL, Yu A, Barrett J, Bomgaars L, Bernstein ML, Blaney SM, and Mondick J (2008) Pharmacokinetics of irinotecan and its metabolites in pediatric cancer patients: a report from the children's oncology group. Cancer Chemother Pharmacol 62:1027-1037.

Thompson PA, Rosner GL, Matthay KK, Moore TB, Bomgaars LR, Ellis KJ, Renbarger J, and Berg SL (2009) Impact of body composition on pharmacokinetics of doxorubicin in children: a Glaser Pediatric Research Network study. Cancer Chemother Pharmacol 64:243-251. 
Pharmacokinetic models for precision dosing in oncology - Supplementary appendix

Titze MI, Schaaf O, Hofmann MH, Sanderson MP, Zahn SK, Quant J, and Lehr T (2017) An allometric pharmacokinetic/pharmacodynamics model for BI 893923, a novel IGF-1 receptor inhibitor. Cancer Chemother Pharmacol 79:545-558.

Toffoli G, Corona G, Sorio R, Robieux I, Basso B, Colussi AM, and Boiocchi M (2001) Population pharmacokinetics and pharmacodynamics of oral etoposide. Br J Clin Pharmacol 52:511-519.

Tornoe CW, Agerso H, Nielsen HA, Madsen H, and Jonsson EN (2004a) Pharmacokinetic/pharmacodynamic modelling of GnRH antagonist degarelix: a comparison of the non-linear mixed-effects programs NONMEM and NLME. $J$ Pharmacokinet Pharmacodyn 31:441-461.

Tornoe CW, Agerso H, Nielsen HA, Madsen H, and Jonsson EN (2004b) Population pharmacokinetic modeling of a subcutaneous depot for GnRH antagonist degarelix. Pharm Res 21:574-584.

Tornoe CW, Agerso H, Senderovitz T, Nielsen HA, Madsen H, Karlsson MO, and Jonsson EN (2007) Population pharmacokinetic/pharmacodynamic (PK/PD) modelling of the hypothalamic-pituitary-gonadal axis following treatment with GnRH analogues. Br J Clin Pharmacol 63:648-664.

Tortorici MA, Cohen EE, Pithavala YK, Garrett M, Ruiz-Garcia A, Kim S, and Fruehauf JP (2014) Pharmacokinetics of single-agent axitinib across multiple solid tumor types. Cancer Chemother Pharmacol 74:1279-1289.

Toshimoto K, Tomaru A, Hosokawa M, and Sugiyama Y (2017) Virtual Clinical Studies to Examine the Probability Distribution of the AUC at Target Tissues Using Physiologically-Based Pharmacokinetic Modeling: Application to Analyses of the Effect of Genetic Polymorphism of Enzymes and Transporters on Irinotecan Induced Side Effects. Pharm Res.

Tout M, Casasnovas O, Meignan M, Lamy T, Morschhauser F, Salles G, Gyan E, Haioun C, Mercier M, Feugier P, Boussetta S, Paintaud G, Ternant D, and Cartron G (2017) Rituximab exposure is influenced by baseline metabolic tumor volume and predicts outcome of DLBCL patients: a Lymphoma Study Association report. Blood 129:2616-2623.

Tracewell WG, Trump DL, Vaughan WP, Smith DC, and Gwilt PR (1995) Population pharmacokinetics of hydroxyurea in cancer patients. Cancer Chemother Pharmacol 35:417-422.

Trame MN, Bergstrand M, Karlsson MO, Boos J, and Hempel G (2011) Population pharmacokinetics of busulfan in children: increased evidence for body surface area and allometric body weight dosing of busulfan in children. Clin Cancer Res 17:6867-6877.

Tranchand B, Amsellem C, Chatelut E, Freyer G, Iliadis A, Ligneau B, Trillet-Lenoir V, Canal P, Lochon I, and Ardiet CJ (1999) A limited-sampling strategy for estimation of etoposide pharmacokinetics in cancer patients. Cancer Chemoth Pharm 43:316-322.

Treiber G, Wex T, Schleyer E, Troeger U, Hosius C, and Malfertheiner P (2008) Imatinib for hepatocellular cancer - Focus on pharmacokinetic/pharmacodynamic modelling and liver function. Cancer Lett 260:146-154.

Urien S, Alexandre J, Raymond E, Brain E, Smith S, Shah A, Cvitkovic E, and Lokiec F (2003a) Phase I population pharmacokinetics of irofulven. Anti-cancer drugs 14:353-358.

Urien S, Fumoleau P, Campone M, Kerbrat P, Bonneterre J, Fargeot P, and Deporte-Fety R (2003b) Modelling of ftorafur and 5-fluorouracil pharmacokinetics following oral UFT administration. A population study in 30 patients with advanced breast cancer. Cancer Chemother Pharmacol 52:99-107.

Urien S, Rezai K, and Lokiec F (2005) Pharmacokinetic modelling of 5-FU production from capecitabine - A population study in 40 adult patients with metastatic cancer. J Pharmacokinet Pharmacodyn 32:817-833. 
Pharmacokinetic models for precision dosing in oncology - Supplementary appendix

Vainas O, Ariad S, Amir O, Mermershtain W, Vainstein V, Kleiman M, Inbar O, Ben-Av R, Mukherjee A, Chan S, and Agur Z (2012) Personalising docetaxel and G-CSF schedules in cancer patients by a clinically validated computational model. Br J Cancer 107:814-822.

Valenzuela B, Nalda-Molina R, Bretcha-Boix P, Escudero-Ortiz V, Duart MJ, Carbonell V, Sureda M, Rebollo JP, Farre J, Brugarolas A, and PerezRuixo JJ (2011) Pharmacokinetic and pharmacodynamic analysis of hyperthermic intraperitoneal oxaliplatin-induced neutropenia in subjects with peritoneal carcinomatosis. AAPS J 13:72-82.

van Erp N, Gelderblom H, van Glabbeke M, Van Oosterom A, Verweij J, Guchelaar HJ, Debiec-Rychter M, Peng B, Blay JY, and Judson I (2008) Effect of cigarette smoking on imatinib in patients in the soft tissue and bone sarcoma group of the EORTC. Clin Cancer Res 14:8308-8313.

van Erp NP, van Herpen CM, de Wit D, Willemsen A, Burger DM, Huitema AD, Kapiteijn E, and Ter Heine R (2016) A Semi-Physiological Population Model to Quantify the Effect of Hematocrit on Everolimus Pharmacokinetics and Pharmacodynamics in Cancer Patients. Clin Pharmacokinet 55:1447-1456.

van Hasselt JG, Gupta A, Hussein Z, Beijnen JH, Schellens JH, and Huitema AD (2013) Population pharmacokinetic-pharmacodynamic analysis for eribulin mesilate-associated neutropenia. Br J Clin Pharmacol 76:412-424.

van Hasselt JG, van Calsteren K, Heyns L, Han S, Mhallem Gziri M, Schellens JH, Beijnen JH, Huitema AD, and Amant F (2014) Optimizing anticancer drug treatment in pregnant cancer patients: pharmacokinetic analysis of gestation-induced changes for doxorubicin, epirubicin, docetaxel and paclitaxel. Annals of oncology : official journal of the European Society for Medical Oncology / ESMO 25:2059-2065.

van Hasselt JGC, Boekhout AH, Beijnen JH, Schellens JHM, and Huitema ADR (2011) Population Pharmacokinetic-Pharmacodynamic Analysis of Trastuzumab-Associated Cardiotoxicity. Clinical pharmacology and therapeutics 90:126-132.

van Kuilenburg ABP, Hausler P, Schalhorn A, Tanck MWT, Proost JH, Terborg C, Behnke D, Schwabe W, Jabschinsky K, and Maring JG (2012) Evaluation of 5-Fluorouracil Pharmacokinetics in Cancer Patients with a c.1905+1G > A Mutation in DPYD by Means of a Bayesian Limited Sampling Strategy. Clin Pharmacokinet 51:163-174.

van Rijswijk REN, Vanloenen AC, Wagstaff J, Meijer E, Lopez R, Vangroeningen CJ, Heimans JJ, and Pinedo HM (1992) Suramin - Rapid Loading and Weekly Maintenance Regimens for Cancer-Patients. Journal of clinical oncology : official journal of the American Society of Clinical Oncology 10:1788-1794.

van Zuylen L, Karlsson MO, Verweij J, Brouwer E, de Bruijn P, Nooter K, Stoter G, and Sparreboom A (2001) Pharmacokinetic modeling of paclitaxel encapsulation in Cremophor EL micelles. Cancer Chemother Pharmacol 47:309-318.

Vogelzang NJ, Mick R, Janisch L, Berezin F, Schilsky RL, and Ratain MJ (1994) Phase-I and Pharmacokinetic Study of a New Antineoplastic Agent Pyrazine Diazohydroxide (Nsc-361456). Cancer Res 54:114-119.

Voller S, Boos J, Krischke M, Wurthwein G, Kontny NE, Boddy AV, and Hempel G (2015) Age-Dependent Pharmacokinetics of Doxorubicin in Children with Cancer. Clin Pharmacokinet 54:1139-1149.

Walsh C, Bonner JJ, Johnson TN, Neuhoff S, Ghazaly EA, Gribben JG, Boddy AV, and Veal GJ (2016) Development of a physiologically based pharmacokinetic model of actinomycin D in children with cancer. Br J Clin Pharmacol 81:989-998. 
Pharmacokinetic models for precision dosing in oncology - Supplementary appendix

Wang E, Nickens DJ, Bello A, Khosravan R, Amantea M, Wilner KD, Parivar K, and Tan W (2016) Clinical Implications of the Pharmacokinetics of Crizotinib in Populations of Patients with Non-Small Cell Lung Cancer. Clinical cancer research : an official journal of the American Association for Cancer Research 22:5722-5728.

Wang K, Stark FS, Schlothauer T, Lahr A, Cosson V, Zhi J, Habben K, Tessier J, Schick E, Staack RF, and Krieter O (2017) An apparent clinical pharmacokinetic drug-drug interaction between bevacizumab and the anti-placental growth factor monoclonal antibody RO5323441 via a target-trapping mechanism. Cancer Chemother Pharmacol 79:661-671.

Wattanatorn W, McLeod HL, Macklon F, Reid M, Kendle KE, and Cassidy J (1997) Comparison of 5-fluorouracil pharmacokinetics in whole blood, plasma, and red blood cells in patients with colorectal cancer. Pharmacotherapy 17:881-886.

Widmer N, Decosterd LA, Csajka C, Leyvraz S, Duchosal MA, Rosselet A, Rochat B, Eap CB, Henry H, Biollaz J, and Buclin T (2006) Population pharmacokinetics of imatinib and the role of alpha-acid glycoprotein. Br J Clin Pharmacol 62:97-112.

Woloch C, Di Paolo A, Marouani H, Bocci G, Ciccolini J, Lacarelle B, Danesi R, and Iliadis A (2012) Population Pharmacokinetic Analysis of 5-FU and 5-FDHU in Colorectal Cancer Patients: Search for Biomarkers Associated with Gastro-Intestinal Toxicity. Current topics in medicinal chemistry 12:1713-1719.

Wong AL, Seng KY, Ong EM, Wang LZ, Oscar H, Cordero MT, Copones R, Fan L, Tan SH, Goh BC, and Lee SC (2014) Body fat composition impacts the hematologic toxicities and pharmacokinetics of doxorubicin in Asian breast cancer patients. Breast cancer research and treatment 144:143152.

Wong H, Gould SE, Budha N, Darbonne WC, Kadel EE, 3rd, La H, Alicke B, Halladay JS, Erickson R, Portera C, Tolcher AW, Infante JR, Mamounas M, Flygare JA, Hop CE, and Fairbrother WJ (2013) Learning and confirming with preclinical studies: modeling and simulation in the discovery of GDC-0917, an inhibitor of apoptosis proteins antagonist. Drug Metab Dispos 41:2104-2113.

Wong M, Balleine RL, Blair EYL, McLachlan AJ, Ackland SP, Garg M, Evans S, Farlow D, Collins M, Rivory LP, Hoskins JM, Mann GJ, Clarke CL, and Gurney H (2006) Predictors of vinorelbine pharmacokinetics and pharmacodynamics in patients with cancer. Journal of clinical oncology : official journal of the American Society of Clinical Oncology 24:2448-2455.

Wu H, Ramanathan RK, Zamboni BA, Strychor S, Ramalingam S, Edwards RP, Friedland DM, Stoller RG, Belani CP, Maruca LJ, Bang YJ, and Zamboni WC (2012a) Mechanism-based model characterizing bidirectional interaction between PEGylated liposomal CKD-602 (S-CKD602) and monocytes in cancer patients. International journal of nanomedicine 7:5555-5564.

Wu K, Cohen EE, House LK, Ramirez J, Zhang W, Ratain MJ, and Bies RR (2012b) Nonlinear population pharmacokinetics of sirolimus in patients with advanced cancer. CPT: pharmacometrics \& systems pharmacology 1:e17.

Xia B, Heimbach T, Lin TH, He H, Wang Y, and Tan E (2012) Novel physiologically based pharmacokinetic modeling of patupilone for human pharmacokinetic predictions. Cancer Chemother Pharmacol 69:1567-1582.

Xiang H, Reyes AE, 2nd, Eppler S, Kelley S, and Damico-Beyer LA (2013) Death receptor 5 agonistic antibody PRO95780: preclinical pharmacokinetics and concentration-effect relationship support clinical dose and regimen selection. Cancer Chemother Pharmacol 72:405-415.

Xie HJ, Griskevicius L, Stahle L, Hassan Z, Yasar U, Rane A, Broberg U, Kimby E, and Hassan M (2006) Pharmacogenetics of cyclophosphamide in patients with hematological malignancies. European Journal of Pharmaceutical Sciences 27:54-61. 
Pharmacokinetic models for precision dosing in oncology - Supplementary appendix

Xie R, Mathijssen RHJ, Sparreboom A, Verweij J, and Karlsson MO (2002) Clinical pharmacokinetics of irinotecan and its metabolites: A population analysis. Journal of clinical oncology : official journal of the American Society of Clinical Oncology 20:3293-3301.

Xin Y, Jin D, Eppler S, Damico-Beyer LA, Joshi A, Davis JD, Kaur S, Nijem I, Bothos J, Peterson A, Patel P, and Bai S (2013) Population pharmacokinetic analysis from phase I and phase II studies of the humanized monovalent antibody, onartuzumab (MetMAb), in patients with advanced solid tumors. $J$ Clin Pharmacol 53:1103-1111.

Xin Y, Li J, Wu J, Kinard R, Weekes CD, Patnaik A, Lorusso P, Brachmann R, Tong RK, Yan Y, Watts R, Bai S, and Hegde PS (2012) Pharmacokinetic and pharmacodynamic analysis of circulating biomarkers of anti-NRP1, a novel antiangiogenesis agent, in two phase I trials in patients with advanced solid tumors. Clinical cancer research : an official journal of the American Association for Cancer Research 18:6040-6048.

Xu L, Wang W, Sheng YC, and Zheng QS (2010) Pharmacokinetics and its relation to toxicity of pegylated-liposomal doxorubicin in Chinese patients with breast tumours. J Clin Pharm Ther 35:593-601.

Yamazaki S, Johnson TR, and Smith BJ (2015) Prediction of Drug-Drug Interactions with Crizotinib as the CYP3A Substrate Using a Physiologically Based Pharmacokinetic Model. Drug metabolism and disposition: the biological fate of chemicals 43:1417-1429.

Yamazaki S, Vicini P, Shen Z, Zou HY, Lee J, Li Q, Christensen JG, Smith BJ, and Shetty B (2012) Pharmacokinetic/pharmacodynamic modeling of crizotinib for anaplastic lymphoma kinase inhibition and antitumor efficacy in human tumor xenograft mouse models. J Pharmacol Exp Ther 340:549-557.

Ye M, Zhu Z, Fu Q, Shen K, and Li DK (2000) Nonlinear pharmacokinetics of paclitaxel in ovarian cancer patients. Acta Pharmacol Sin 21:596-599.

Yoshiba S, Jansen M, Matsushima N, Chen S, and Mendell J (2016) Population pharmacokinetic analysis of patritumab, a HER3 inhibitor, in subjects with advanced non-small cell lung cancer (NSCLC) or solid tumors. Cancer Chemother Pharmacol 77:987-996.

Yoshida K, Maeda K, Kusuhara H, and Konagaya A (2013) Estimation of feasible solution space using Cluster Newton Method: application to pharmacokinetic analysis of irinotecan with physiologically-based pharmacokinetic models. BMC systems biology 7 Suppl 3:S3.

You B, Salles G, Bachy E, Casasnovas O, Tilly H, Ribrag V, Sebban C, Henin E, Guitton J, Tod M, and Freyer G (2015) Etoposide pharmacokinetics impact the outcomes of lymphoma patients treated with BEAM regimen and ASCT: a multicenter study of the LYmphoma Study Association (LYSA). Cancer Chemother Pharmacol 76:939-948.

You B, Tranchand B, Girard P, Falandry C, Ribba B, Chabaud S, Souquet PJ, Court-Fortune I, Trillet-Lenoir V, Fournel C, Tod M, and Freyer G (2008) Etoposide pharmacokinetics and survival in patients with small cell lung cancer: a multicentre study. Lung cancer 62:261-272.

Yu H, Steeghs N, Kloth JS, de Wit D, van Hasselt JG, van Erp NP, Beijnen JH, Schellens JH, Mathijssen RH, and Huitema AD (2015) Integrated semiphysiological pharmacokinetic model for both sunitinib and its active metabolite SU12662. Br J Clin Pharmacol 79:809-819.

Yu H, van Erp N, Bins S, Mathijssen RH, Schellens JH, Beijnen JH, Steeghs N, and Huitema AD (2017a) Development of a Pharmacokinetic Model to Describe the Complex Pharmacokinetics of Pazopanib in Cancer Patients. Clin Pharmacokinet 56:293-303.

Yu M, Gao Z, Dai X, Gong H, Zhang L, Chen X, Zhong DF, and Sy SK (2017b) Population Pharmacokinetic and Covariate Analysis of Apatinib, an Oral Tyrosine Kinase Inhibitor, in Healthy Volunteers and Patients with Solid Tumors. Clin Pharmacokinet 56:65-76. 
Pharmacokinetic models for precision dosing in oncology - Supplementary appendix

Zamboni WC, D'Argenio DZ, Stewart CF, MacVittie T, Delauter BJ, Farese AM, Potter DM, Kubat NM, Tubergen D, and Egorin MJ (2001) Pharmacodynamic model of topotecan-induced time course of neutropenia. Clinical cancer research : an official journal of the American Association for Cancer Research 7:2301-2308.

Zandvliet AS, Huitema AD, Copalu W, Yamada Y, Tamura T, Beijnen JH, and Schellens JH (2007) CYP2C9 and CYP2C19 polymorphic forms are related to increased indisulam exposure and higher risk of severe hematologic toxicity. Clin Cancer Res 13:2970-2976.

Zandvliet AS, Schellens JHM, Copalu W, Beijnen JH, and Huitema ADR (2006) A semi-physiological population pharmacokinetic model describing the non-linear disposition of indisulam. J Pharmacokinet Phar 33:543-570.

Zao JH, Schechter T, Liu WJ, Gerges S, Gassas A, Egeler RM, Grunebaum E, and Dupuis LL (2015) Performance of Busulfan Dosing Guidelines for Pediatric Hematopoietic Stem Cell Transplant Conditioning. Biol Blood Marrow Tr 21:1471-1478.

Zhu M, Doshi S, Gisleskog PO, Oliner KS, Perez Ruixo JJ, Loh E, and Zhang Y (2014) Population pharmacokinetics of rilotumumab, a fully human monoclonal antibody against hepatocyte growth factor, in cancer patients. J Pharm Sci 103:328-336.

Zuhlsdorf M, Bhattaram VA, Campioni M, Krosser S, Derendorf H, and Kovar A (2014) Population pharmacokinetics of cilengitide in adult and pediatric cancer patients from a nonlinear mixed-effects analysis. J Clin Pharmacol 54:1391-1399. 